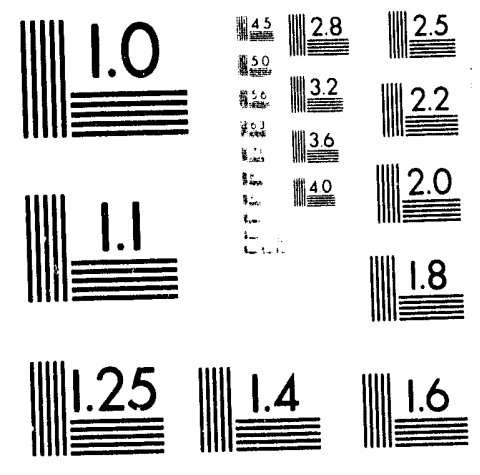



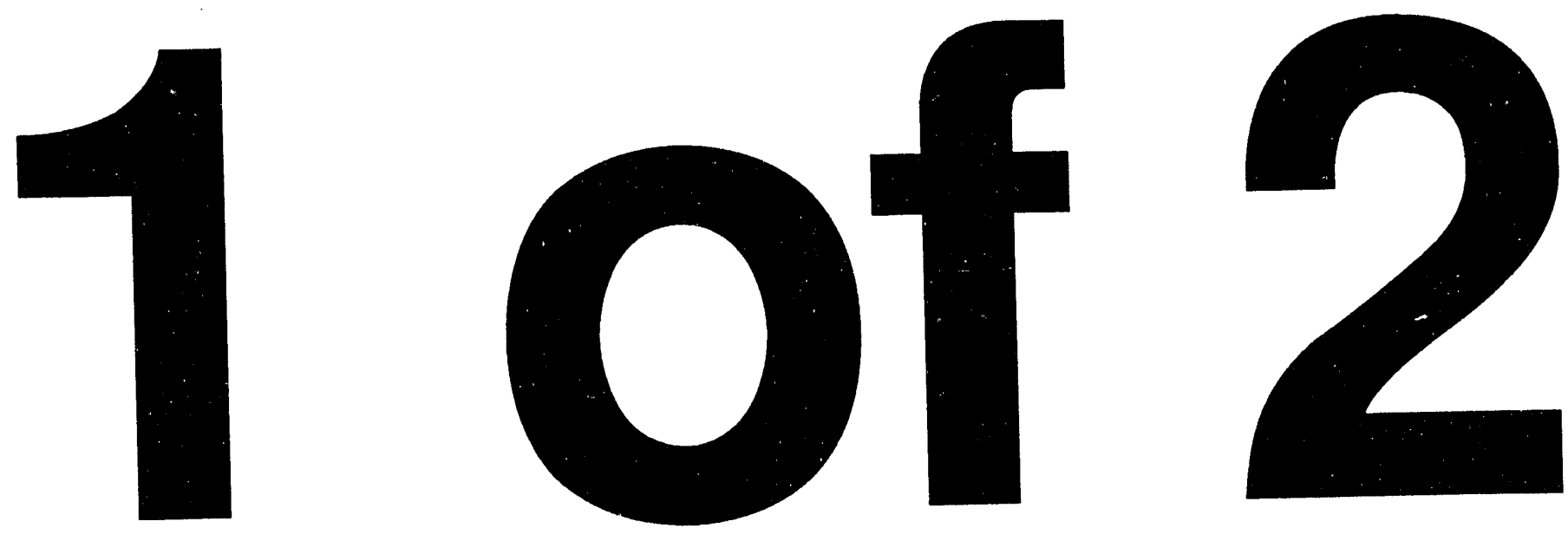


\section{Characterization of the Geology, Geochemistry, and Microbiology of the Radio Frequency Heating Demonstration Site at the Savannah River Site ${ }^{(U)}$}

Prepared for the U.S. Department of Energy under contract no. DE-AC09-89SR1 8035 


\section{Disclaimer}

This report was prepared as an account of work sponsored by an agency of the United States Government. Neither the United States Government nor any agency thereof, nor any of their employees, makes any warranty, express or implied, or assumes any legal liability or responsibility for the accuracy, completeness, or usefulness of any information, apparatus, product, or process disclosed, or represents that its use would not infringe privately owned rights. Reference herein to any specific commercial product, process, or service by trade name, trademark, manufacturer, or otherwise does not necessarily constitute or imply its endorsement, recommendation, or favoring by the United States Government or any agency thereof. The views and opinions of authors expressed herein do not necessarily state or reflect those of the United States Government or any agency thereof. 


\section{Contents}

Objective 1

Introduction 1

Geology and Stratigraphy 2

Tobacco Road Sand 3

Upland Unit 3

Demonstration Site Description 4

Sampling and Analysis of Sediment Samples 9

In Situ Moisture Content 9

Microbiology 11

Contamination Distribution 12

Volumetric Calculations 13

References 19

Appendix 1-Core Descriptions 20

Appendix II--Moisture Content or Soil Water

from Six Sediment Cores 21

Appendix III--Tabulated TCE and PCE for

Pre- and Post-test Borings 22

Appendix IV-_Plots of TCE and PCE vs. Depth 23 


\title{
Characterization of the Geology, Geochemistry, and Microbiology of the Radio Frequency Heating Demonstration Site at the Savannah River Site
}

\author{
C. A. Eddy Dilek, T. R. Jarosch, C. B. Fliermans, B. B. Looney, and W. H. Parker \\ Savannah River Technology Center \\ Westinghouse Savannah River Company \\ Aiken, SC 29802
}

\section{Objective}

The overall objective of the Integrated Demonstration Project for the Remediation of Organics at Nonarid Sites at the Savannah River Site (SRS) is to evaluate innovative remediation, characterization, and monitoring systems to facilitate restoration of contaminated sites. The first phase of the demonstration focused on the application and development of in situ air stripping technologies to remediate sediments and groundwater contaminated with volatile organic compounds (VOCs). The second phase focused on the enhancement of the in situ air stripping process by adding selected nutrients to stimulate naturally occurring microorganisms that degrade VOCs. The purpose of the third phase was to evaluate the use of heating technologies [radio frequency (RF) and ohmic heating] io enhance the removal of contamination from clay layers where mass transfer is limited.

The objective of this report is to document pretest and post-test data collected in support of the RF heating demonstration. The following data are discussed in this report:

- a general description of the site including piezometers and sensors installed to monitor the remedial process

- stratigraphy, lithology, and a detailed geologic cross section of the study site

- tabulations of pretest and post-test moisture and VOC content of the sediments

- sampling and analysis procedures for sediment samples

- microbial abundance and diversity

- three-dimensional images of pretest and post-test contaminant distribution

- volumetric calculations

The description of the remedial process, detailed modeling and performance evaluation, and results from fiber optic temperature sensor data will be documented in separate reports.

\section{Introduction}

Vadose zone characterization data collected previously as part of the Integrated Demonstration Project at SRS have clearly identified a shallow clay layer $(30-45 \mathrm{ft}$ deep) located below a portion of the abandoned process sewer line between the M-Area Metal Fabrication Facility and the M-Area Seepage Basin that contains significant quantities of VOCs (WSRC-RP-90-1335 and WSRC-RD-9122). Analysis of sediment samples collected during installation of characterization wells have identified both trichloroethylene (TCE) and perchloroethylene (PCE) within the clay zone at elevated levels relative to the surrounding sandy sediments. The slow release of solvent from this clay provides a long-term source for groundwater contamination. While vacuum extraction can remove the contaminants from the clay before they reach the groundwater, this method is still limited by mass transfer out of the clay, resulting in unacceptably long remedial campaigns. Heating the clays to increase removal of the VOCs in conjunction with vacuum extraction is one of the most promising in situ treatment options. By selectively heating the clays to temperatures at or above $100^{\circ} \mathrm{C}$, the release and transport of TCE and PCE will be enhanced as a result of several factors, including the following:

- an increase in the contaminant vapor pressure and diffusivity

- an increase in the effective porosity of the sediment with the release of water vapor and contaminant

- enhanced removal from in situ steam stripping by the water vapor generated from heating to temperatures in excess of $100^{\circ} \mathrm{C}$

Other methods of subsurface heating (e.g., hot air or steam injection) are poorly suited to heating clay layers because of the low permeability of clay. 
The purpose of this field dernonstration was to test and evaluate the enhanced remov'al of chlorinated VOCs from subsurface sediments using in situ RF heating. A horizontal well was installed in the clay zone using an innovative directional drilling technique developed by Charles Machine Works (Ditch Witch $\$$ ). A fiberglass, epoxy line pipe casing, approximately $600 \mathrm{ft}$ long with $300 \mathrm{ft}$ of slotted fiberglass well screen, was installed. The installation procedure and well construction of the horizontal well are described in detail in a separate report (WSRC-TR-93. 008). An antenna that emits RF radiation was placed in the horizontal well. The dielectric properties of the contaminated clay zones preferentially capture the RF energy, focusing the applied power precisely in the zones that need to be heated. The RF heating project used a dipole applicator developed and patented by KAI Tech., Inc. The single wand applicator is approximately 17 feet in length and operates at $13.56 \mathrm{MHz}$ with a maximum power output of $25 \mathrm{~kW}$. During the heating, the same horizontal well was used for vacuum extraction.

To assess the effectiveness of the heating technology, predemonstration and post-demonstration sediment cores were collected from boreholes within and surrounding the heated zone and in a cold control zone (WSRC-RP-921428). Lithologic description of the cores were used to generate cross sections to confirm the location of the targeted clay and to aid in the interpretation of subsurface temperature and pressure patterns. Samples from the cores were analyzed for the following:

- chlorinated volatile organic content to determine changes in the residual contaminant concentrations

- moisture content to determine potential changes in effective permeability to gas flow and to aid in the calculations of RF absorption efficiency

- microbial community population to determine the temperature regimes which accelerate growth or kill subsurface organisms

Three-dimensional images of pre- and post-distribution of PCE and TCE were prepared and contaminant inventories were estimated from the images.

\section{Geology and Stratigraphy}

SRS is located on the Atlantic Coastal Plain. The Coastal Plain sediments are approximately 1000 feet thick at SRS, and are dominantly composed of stratified clay, sand, and gravel. In general, these sediments dip gently to the southeast. Brief lithologic descriptions of the stratigraphic units from the surface to a 200- $\mathrm{ft}$ depth at the Integrated Demonstration Site (IDS) are available in Eddy Dilek et al. (1993). The unsaturated sediments within the top $60 \mathrm{ft}$ are of interest to this study. They consist of sands, sandy clays, clayey sands, and clays deposited in Late Eocene to Miocene.

\section{Methods}

Core samples were described in the WSRC core logging facility using a standardized procedure for the description of core material. A sample of core material is homogenized over a one-foot interval and described (Procedure ESSOP-2-15: Microscopic Examination of Sediment Cores). Descriptions made using this procedure ignore lithologic features less than a foot in thickness and reflect a "whole-rock" lithologic classification of the sediment. For example, an interval containing $40 \%$ sandsized material and $60 \%$ mud-sized material in discrete interbeds would be classified as a sandy clay. An interval containing the same percentage distributions but with the mud fraction dispersed throughout the interval as matrix would also be classified as a sandy clay. These intervals may appear identical on a geologic cross section despite their differences in depositional environment and hydrologic properties.

Two hydrogeologic cross sections across the IDS were constructed along north-south and east-west trends, intersecting at well MHV21. A total of 10 borings was used to construct the cross sections (Table 1). Continuous core description and core samples are available for all of the wells. Geophysical logs were only available for the deepest well MHT16C, which penetrated to a depth of $200 \mathrm{ft}$. The cross sections presented in this report are tied by well MHT16C to cross sections prepared for earlier phases of the Integrated Demonstration Project (Eddy Dilek 1993).

Individual sediment types represented on the cross sections may appear very different because of symbol and color change; however, the variations in lithology are relaiively continuous. Examples of this are common in the Upland Unit, which consists of both sandy clays with a high sand content and clayey sands with a high mud content. A clayey sand composed of $45 \%$ mud matrix is very similar to a sandy clay composed of $55 \%$ mud matrix. The clayey sand will be yellow on the cross section and the sandy clay will be green. This gives the impression tha: these sediments have different properties and the boundary represents a sharp lithologic change when in fact the change is gradational.

Because of previous construction projects in the study area, including removal of the process sewer line, the surficial 5-10 ft of sediments have been disturbed. It is difficult to distinguish between this fill material and the sediments because of the coarse texture and massive bed- 
ding of the Upland Unit, so correlations in the surficial sediment should be interpreted with caution.

\section{Discussion}

Lithostratigraphic units identified during the core-logging process include the Upland Unit and Tobacco Roar Sand.

\section{Tobacco Road Sand}

The Tobacco Road Sand consists of light grayish purple to moderate grayish purple, medium- to fine-grained sand to clayey sand with approximately $10-15 \%$ interstitial mud. The top of the Tobacco Road Sand is marked by a moderate grayish purple clay to clayey sand that varies in thickness from 18 to $6 \mathrm{ft}$. The sandy portion of the unit tends to contain more clay (up to $30 \%$ ) at the base. The unconformable contact with the overlying Upland Unit is very irregular, suggesting heavy scouring of the Tobacco Road Sand during deposition of the Upland Unit.

\section{Upland Unit}

The Upland Unit is reddish orange to brown to purple with a highly variable lithology. The unit ranges from poorly sorted, gravely sand to clay that contains virtually no sand. The unit has a distinctive speckled appearance in sandier layers. The contact of this unit with the underlying Tobacco Road Sand is unconformable and highly irregular.

Gravel-sized clay balls are common in the Upland Unit. Moderate grayish purple clay balls of boulder size have been observed in core samples. Boulder-sized clay balls in the core material were identified and differentiated from individual clay layers based on the following criteria and observations:

- The clay balls have a light yellowish orange weathering rind.

- The sediment has a relatively coarse texture near the clay balls.

- Numerous cobble and pebble-sized clay balls associated with the boulder size clasts.

- Clay balls at the base of the Upland Unit are commonly seen in outcrop. This outcrop exposing boulder size purple clay balls is found on the north side of Road F just southeast of Upper Three Runs at SRS. These clay balls are probably reworked from a clay layer of similar lithology at the top of the Tobacco Road Sand.
The highly interbedded sediments govern gas and fluid flow in the subsurface at the IDS. The water table is located at an elevation of approximately $230 \mathrm{ft}$ above mean sea level (msl) (a depth of approximately 130-140 ft below surface). The sitewide and local hydrostratigraphy, subsurface hydrologic properties, hydrologic conditions, and vadose zone properties at the IDS are addressed in the pre- and post-test characterization reports (WSRC-RD-9121, WSRC-TR-93-369). In these reports, four semi-confining/confining zones in the vadose and saturated zone at the IDS are delineated, named for the approximate elevations at which they were found (Eddy et al. 1991). These are, from the surface down, the "325-ft clay", the "300-ft clay", the "270-ft clay" ("tan clay"), and the "200-ft clay" ("green clay") zones. The "325-ft clay" and the "300-ft clay" are in the vadose zone, impacting the flow of gases above the water table and recharge of air and water from the ground surface.

The "325-ft clay" is the unit that was targeted in the RF heating study. It varies in thickness significantly across the RF heating demonstration site from 5.3 to $17.6 \mathrm{ft}$. It consists of sediments assigned to the Tobacco Road Sand. The majority of the clay and sandy clay in the unit consist of interbedded sand and clay. The estimated porosity (per ESSOP-2-15) ranges from poor in the clay a.7d sandy clay to good in interbedded sand.

There are two contributing factors for the significant variations in thickness of the " $325-\mathrm{ft}$ clay", the erosional unconformity at the top of the Tobacco Road Sand and variations in depositional facies. The variations due to the erosional unconformity at the base of the Upland Unit are minor. However, the combination of the two can greatly reduce the thickness of this unit over a very short distance. This is observed in MHV-22, where this unit is only $5.3 \mathrm{ft}$ in thickness. Core description data shows that the "325-ft clay" comprises layers of clayey sand, sandy clay, and clay, with subordinate sand layers. Hydrostratigraphic picks for these zones determined from the cross sections are summarized in Table 1.

The lithostratigraphy of the IDS is illustrated in detail on two hydrogeologic cross sections (Plates ??). The "325-ft clay" varies greatly in thickness in a relatively short distance, but it is a continuous confining layer across the site. The cross sections illustrate the intercalated geology of the confining zones and demonstrate the overall discontinuity of the individual clayey layers within each zone. 
Table 1. Picks for the "325-ft. Clay" Confining Zone Used in Construction of Cross Sections

\begin{tabular}{|c|c|c|c|c|c|c|c|}
\hline Well I.D. & $\begin{array}{l}\text { Total } \\
\text { Core } \\
\text { (ft) }\end{array}$ & $\begin{array}{l}\text { Ground } \\
\text { Elevation* } \\
\text { (n msl) }\end{array}$ & $\begin{array}{l}\text { Depth } \\
\text { Top } \\
\text { (ft) }\end{array}$ & $\begin{array}{l}\text { Depth } \\
\text { Bottom } \\
\text { (ft) }\end{array}$ & $\begin{array}{c}\text { Elevation } \\
\text { Top } \\
\text { (nt msl) }\end{array}$ & $\begin{array}{c}\text { Elevation } \\
\text { Bottom } \\
\text { (f msl) }\end{array}$ & $\begin{array}{c}\text { Unit } \\
\text { Thickness } \\
\text { (ft) }\end{array}$ \\
\hline MHT-16Cb & 200 & 359.1 & 36.0 & 47.0 & 323.1 & 312.1 & 11.0 \\
\hline MHV-20a & 60 & 359.3 & 36.0 & 46.0 & 323.3 & 313.3 & 10.0 \\
\hline MHV-21a & 60 & 359.2 & 34.0 & 46.0 & 325.2 & 313.2 & 12.0 \\
\hline MHV-22a & 60 & 359.2 & 34.7 & 40.0 & 324.5 & 319.2 & 5.3 \\
\hline MHV-23a & 60 & 359.2 & 35.7 & 46.0 & 323.5 & 313.2 & 10.3 \\
\hline MHV-24a & 60 & 360.0 & 32.0 & 43.0 & 328.0 & 317.0 & 11.0 \\
\hline MHV-25a & 60 & 359.0 & 34.0 & 46.0 & 325.0 & 313.0 & 12.0 \\
\hline MHV-26a & 60 & 359.4 & 31.0 & 46.0 & 328.4 & 313.4 & 15.0 \\
\hline MHV-27a & 60 & 359.5 & 32.4 & 50.0 & 327.1 & 309.5 & 17.6 \\
\hline MHV-28a & 60 & 359.5 & 34.3 & 45.0 & 325.2 & 314.5 & 10.7 \\
\hline
\end{tabular}

* Ground Elevations used for MHV wells were surveyed stake positions (11/6/92).

\section{Demonstration Site Description}

\section{Wells and Borings}

The nature and extent of solvent contamination at the IDS was determined by collecting sediment samples from 10 borings (designated by the prefix MHV). Five borings were installed parallel approximately $10 \mathrm{ft}$ away from the horizontal well AMH5 (Figure 1). An additional two locations in line with MHV21 were installed perpendicular to the trend of the horizontal well. Three additional locations were chosen outside the predicted zone of heating to serve as control locations. Since the target clay layer (" $325-\mathrm{ft}$ clay") that was heated is located at depths from 30 to $45 \mathrm{ft}_{\text {? }}$ the borings were drilled with a hollow stem auger to a nominal 60 -ft depth to characterize contamination above, within, and below the clay. Continuous sediment cores were collected with a split-spoon sampler starting at approximately $25 \mathrm{ft}$ to total depth. Samples were collected at selected intervals from the cores in the field for chemical and microbial analysis, and determination of moisture content. Geologic descriptions of the core were prepared in the field (Appendix I).

Multiple characterization and monitoring devices were installed. In each of the 10 locations, a piezometer cluster was installed that consisted of three piezometers, each consisting of 1 -inch schedule 40 polyvinyl chloride casing with a 5 -ft screened interval at the bottom (Table 2). The tops of 5-ft screens were set at 28,38 , and 48 feet in each cluster to monitor contaminant concentrations and pres- sures above, within, and below the clay layer. Well construction diagrams for the piezometers are provided in Appendix I.

A second borehole was installed next to each piezometer cluster and an array of thermocouples, fiber optic temperature sensors, and electrodes for electrical resistance tomography were installed (Table 3). These locations are designated by the well number of the associated piezometer cluster and are designated with the suff $x \mathrm{~T}$. For example, the temperature sensors adjacent piezometer MHV22 would be MHV22T (Figure 1). Three thermocouples were placed in each location at approximately 28,38 and 48 fect depth. Well construction details for these installations are given in Appendix I. The installation and operation of fiber optic temperature sensors and electric resistance tomography electrodes will be presented in separate reports.

For the post-test characterization effort, cores were collected, if possible, adjacent to each of the MHV clusters. Figure 1 shows the location of pre- and post-test borings. Three of the cores, one core from the control zone (MHB28V) and two from the heated zone (MHB21V and MHB24V), were collected immediately after the antenna was turned off and the ground was still hot. This was done to collect samples that would have microbial assemblages and VOC contents representative of peak heating conditions. After a 14-day cool-down period, the remaining seven cores were collected to obtain the rest of the sediment samples. 
Radio Frequency Heating Demonstration Site Characterization

Table 2. Construction Details for MHV Piezometer Clusters

\begin{tabular}{|c|c|c|c|c|c|c|c|c|}
\hline Well ID & SRS North & SRS East & TOC & $\begin{array}{l}\text { Depth } \\
\text { Screen }\end{array}$ & $\begin{array}{l}\text { Screen } \\
\text { Length }\end{array}$ & $\begin{array}{l}\text { Top of } \\
\text { Screen }\end{array}$ & $\begin{array}{l}\text { Bottom of } \\
\text { Screen }\end{array}$ & $\begin{array}{c}\text { Pad } \\
\text { Elevation }\end{array}$ \\
\hline MHV 20A & 102418.35 & 48658.30 & 361.75 & 48 & 4.90 & 312.35 & 307.45 & 359.6 \\
\hline MHV 20B & 102418.35 & 48658.30 & 361.74 & 38 & 4.89 & 322.14 & 317.25 & 359.6 \\
\hline MHV $20 \mathrm{C}$ & 102418.35 & 48658.30 & 361.74 & 28 & 4.90 & 331.94 & 327.04 & 359.6 \\
\hline MHV 21A & 102433.61 & 48661.47 & 361.95 & 48 & 4.90 & 312.45 & 307.55 & 359.7 \\
\hline MHV 21B & 102433.61 & 48661.47 & 361.96 & 38 & 4.90 & 322.36 & 317.46 & 359.7 \\
\hline MHV 21C & 102433.61 & 48661.47 & 361.97 & 28 & 4.90 & 332.27 & 327.37 & 359.7 \\
\hline MHV 22A & 102443.46 & 48663.79 & 361.95 & 48 & 4.91 & 312.45 & 307.54 & 359.7 \\
\hline MHV 22B & 102443.46 & 48663.79 & 361.97 & 38 & 4.89 & 322.27 & 317.38 & 359.7 \\
\hline MHV 22C & 102443.46 & 48663.79 & 361.95 & 28 & 4.89 & 332.25 & 327.36 & 359.7 \\
\hline MHV 23A & 102458.02 & 48666.89 & 361.93 & 48 & 4.89 & 312.46 & & 359.7 \\
\hline MHV 23B & 102458.02 & 48666.89 & 361.91 & 38 & 4.89 & 322.34 & 317.45 & 359.7 \\
\hline MHV 23C & 102458.02 & 48666.89 & 361.95 & 28 & 4.89 & 332.32 & 327.43 & 359.7 \\
\hline MHV 24A & 102439.80 & 48636.88 & 362.74 & 48 & 4.90 & 313.34 & 308.44 & 360.5 \\
\hline MHV 24B & 102439.80 & 48636.88 & 362.72 & 38 & 4.89 & 323.12 & 318.23 & 360.5 \\
\hline MHV $24 \mathrm{C}$ & 102439.80 & 48636.88 & 362.76 & 28 & 4.89 & 333.06 & 328.17 & 360.5 \\
\hline MHV 25A & 102428.94 & 48680.95 & 361.46 & 48 & 4.90 & 311.96 & 307.06 & 359.1 \\
\hline MHV 25B & 102428.94 & & 361.47 & 38 & 4.90 & 321.87 & 316.97 & 359.1 \\
\hline MHV $25 \mathrm{C}$ & 102428.94 & 48680.95 & 361.47 & 28 & 4.89 & 331.77 & 326.88 & 359.1 \\
\hline MHV 26A & 102404.20 & 48655.31 & 362.13 & 48 & 4.91 & 312.53 & 307.62 & 359.8 \\
\hline MHV 26B & 102404.20 & 48655.31 & 362.13 & 38 & 4.89 & 322.53 & 317.64 & 359.8 \\
\hline MHV $26 \mathrm{C}$ & 102404.20 & 48655.31 & 362.09 & 28 & 4.90 & 332.39 & 327.49 & 359.8 \\
\hline MHV 27A & 102374.32 & 48654.50 & 362.05 & 48 & 4.90 & 312.45 & 307.55 & 359.9 \\
\hline MHV 27B & 102374.32 & 48654.50 & 362.04 & 38 & 4.89 & 322.44 & 317.55 & 359.9 \\
\hline MHV 27C & 102374.32 & 48654.50 & 362.05 & 28 & 4.90 & 332.35 & 327.45 & 359.9 \\
\hline MHV 28A & 102366.27 & 48647.61 & 362.22 & 48 & 4.89 & 312.72 & 307.83 & 359.9 \\
\hline MHV 28B & 102366.27 & 48647.61 & 362.24 & 38 & 4.90 & 322.64 & 317.74 & 359.9 \\
\hline MHV $28 \mathrm{C}$ & 102366.27 & 48647.61 & 362.22 & 28 & 4.89 & 332.52 & 327.63 & 359.9 \\
\hline MHV 29A & 102362.22 & 48661.78 & 361.80 & 48 & 4.91 & 312.30 & 307.39 & 359.6 \\
\hline MHV 29B & 102362.22 & 48661.78 & 361.79 & 38 & 4.90 & 322.19 & 317.29 & 359.6 \\
\hline MHV 29C & 102362.22 & 48661.78 & 361.80 & 28 & 4.89 & 332.00 & 327.11 & 359.6 \\
\hline
\end{tabular}


Table 3. Construction Detaiis for M:IV-T Sensors

\begin{tabular}{|c|c|c|c|c|c|c|c|c|c|}
\hline Well ID & SRS North & SRS East & $\begin{array}{c}\text { Pad } \\
\text { elevation }\end{array}$ & $\begin{array}{c}\text { Fiber Optic } \\
\text { depth }\end{array}$ & $\begin{array}{c}\text { Thermocouple } \\
\text { depth }\end{array}$ & $\begin{array}{l}\text { Electrode } \\
\text { depth }\end{array}$ & $\begin{array}{l}\text { Fiber Optic } \\
\text { elevation }\end{array}$ & $\begin{array}{c}\text { Thermocouple } \\
\text { elevation }\end{array}$ & $\begin{array}{l}\text { Electrode } \\
\text { elevation }\end{array}$ \\
\hline \multirow[t]{6}{*}{ MHV 20T } & 102420.47 & 48649.84 & 359.9 & 28.0 & 28.0 & none & 331.65 & 331.70 & none \\
\hline & & & 359.9 & 33.0 & 38.0 & & 326.65 & 321.70 & \\
\hline & & & 359.9 & 38.0 & 48.0 & & 321.65 & 311.70 & \\
\hline & & & 359.9 & 43.0 & & & 316.65 & & \\
\hline & & & 359.9 & 48.0 & & & 311.65 & & \\
\hline & & & 359.9 & 53.0 & & & 306.65 & & \\
\hline \multirow[t]{10}{*}{ MHV 21T } & 102435.54 & 48653.51 & 360.0 & 28.0 & 28.0 & 15.0 & 331.80 & 331.75 & 344.75 \\
\hline & & & 360.0 & 33.0 & 38.0 & 20.0 & 326.80 & 321.75 & 339.75 \\
\hline & & & 360.0 & 38.0 & 48.0 & 25.0 & 321.80 & 311.75 & 334.75 \\
\hline & & & 360.0 & 43.0 & & 30.0 & 316.80 & & 329.75 \\
\hline & & & 360.0 & 48.0 & & 35.0 & 311.80 & & 324.75 \\
\hline & & & 360.0 & 53.0 & & 40.0 & 306.80 & & 319.75 \\
\hline & & & 360.0 & & & 45.0 & & & 314.75 \\
\hline & & & 360.0 & & & 50.0 & & & 309.75 \\
\hline & & & 360.0 & & & 55.0 & & & 304.75 \\
\hline & & & 360.0 & & & 60.0 & & & 299.75 \\
\hline \multirow[t]{6}{*}{ MHV 22T } & 102446.01 & 48657.10 & 360.0 & 28.0 & 28.0 & none & 331.80 & 331.75 & none \\
\hline & & & 360.0 & 33.0 & 38.0 & & 326.80 & 321.75 & \\
\hline & & & 360.0 & 38.0 & 48.0 & & 321.80 & 311.75 & \\
\hline & & & 360.0 & 43.0 & & & 316.80 & & \\
\hline & & & 360.0 & 48.0 & & & 311.80 & & \\
\hline & & & 360.0 & 53.0 & & & 306.80 & & \\
\hline \multirow[t]{6}{*}{ MHV 23T } & 102460.80 & 48661.00 & 359.9 & 28.0 & 28.0 & none & 331.70 & 331.65 & none \\
\hline & & & 359.9 & 33.0 & 38.0 & & 326.70 & 321.65 & \\
\hline & & & 359.9 & 38.0 & 48.0 & & 321.70 & 311.65 & \\
\hline & & & 359.9 & 43.0 & & & 316.70 & & \\
\hline & & & 359.9 & 48.0 & & & 311.70 & & \\
\hline & & & 359.9 & 53.0 & & & 306.70 & & \\
\hline \multirow[t]{10}{*}{ MHV 24T } & 102442.60 & 48640.86 & 360.4 & 28.0 & 28.0 & 15.0 & 332.20 & 332.15 & 345.15 \\
\hline & & & 360.4 & 33.0 & 38.0 & 20.0 & 327.20 & 322.15 & 340.15 \\
\hline & & & 360.4 & 38.0 & 48.0 & 25.0 & 322.20 & 312.15 & 335.15 \\
\hline & & & 360.4 & 43.0 & & 30.0 & 317.20 & & 330.15 \\
\hline & & & 360.4 & 48.0 & & 35.0 & 312.20 & & 325.15 \\
\hline & & & 360.4 & 53.0 & & 40.0 & 307.20 & & 320.15 \\
\hline & & & 360.4 & & & 45.0 & & & 315.15 \\
\hline & & & 360.4 & & & 50.0 & & & 310.15 \\
\hline & & & 360.4 & & & 55.0 & & & 305.15 \\
\hline & & & 360.4 & & & 60.0 & & & 300.15 \\
\hline
\end{tabular}


Radio Frequency Heating Demonstration Site Characterization

\begin{tabular}{|c|c|c|c|c|c|c|c|c|c|}
\hline Well ID & SRS North & SRS East & $\begin{array}{c}\text { Pad } \\
\text { elevation }\end{array}$ & $\begin{array}{l}\text { Fiber Optic } \\
\text { depth }\end{array}$ & $\begin{array}{c}\text { Thermocouple } \\
\text { depth }\end{array}$ & $\begin{array}{l}\text { Electrode } \\
\text { depth }\end{array}$ & $\begin{array}{l}\text { Flber Optic } \\
\text { elevation }\end{array}$ & $\begin{array}{c}\text { Thermocouple } \\
\text { elevation }\end{array}$ & $\begin{array}{l}\text { Electrode } \\
\text { elevation }\end{array}$ \\
\hline \multirow[t]{10}{*}{ MHV 25T } & 102424.54 & 48679.01 & 359.0 & 28.0 & 28.0 & 15.0 & 330.80 & 330.75 & 343.75 \\
\hline & & & 359.0 & 38.0 & 38.0 & 20.0 & 320.80 & 320.75 & 338.75 \\
\hline & & & 359.0 & 48.0 & 48.0 & 25.0 & 310.80 & 310.75 & 333.75 \\
\hline & & & 359.0 & & & 30.0 & & & 328.75 \\
\hline & & & 359.0 & & & 35.0 & & & 323.75 \\
\hline & & & 359.0 & & & 40.0 & & & 318.75 \\
\hline & & & 359.0 & & & 45.0 & & & 313.75 \\
\hline & & & 359.0 & & & 50.0 & & & 308.75 \\
\hline & & & 359.0 & & & 55.0 & & & 303.75 \\
\hline & & & 359.0 & & & 60.0 & & & 298.75 \\
\hline \multirow[t]{3}{*}{ MHV 26T } & 102406.07 & 48647.86 & 359.9 & 28.0 & 28.0 & none & 331.70 & 331.65 & none \\
\hline & & & 359.9 & 38.0 & 38.0 & & 321.70 & 321.65 & \\
\hline & & & 359.9 & 48.0 & 48.0 & & 311.70 & 311.65 & \\
\hline \multirow[t]{3}{*}{ MHV 2TT } & 102380.37 & 48650.30 & 359.9 & 28.0 & 28.0 & none & 331.70 & 331.65 & none \\
\hline & & & 359.9 & 38.0 & 38.0 & & 321.70 & 321.65 & \\
\hline & & & 359.9 & 48.0 & 48.0 & & 311.70 & 311.65 & \\
\hline \multirow[t]{3}{*}{ MHV 28T } & 102367.03 & 48642.91 & 360.0 & 28.0 & 28.0 & none & 331.75 & 331.75 & none \\
\hline & & & 360.0 & 38.0 & 38.0 & & 321.75 & 321.75 & \\
\hline & & & 360.0 & 48.0 & 48.0 & & 311.75 & 311.75 & \\
\hline \multirow[t]{3}{*}{ MHV 29T } & 102363.75 & 48656.42 & 359.8 & 28.0 & none & none & 331.60 & none & none \\
\hline & & & 359.8 & 38.0 & & & $321.60)$ & & \\
\hline & & & 359.8 & 48.0 & & & 311.60 & & \\
\hline
\end{tabular}


Figure 1. Well and Boring Locations for the Radio Frequency Heating Demonstration 


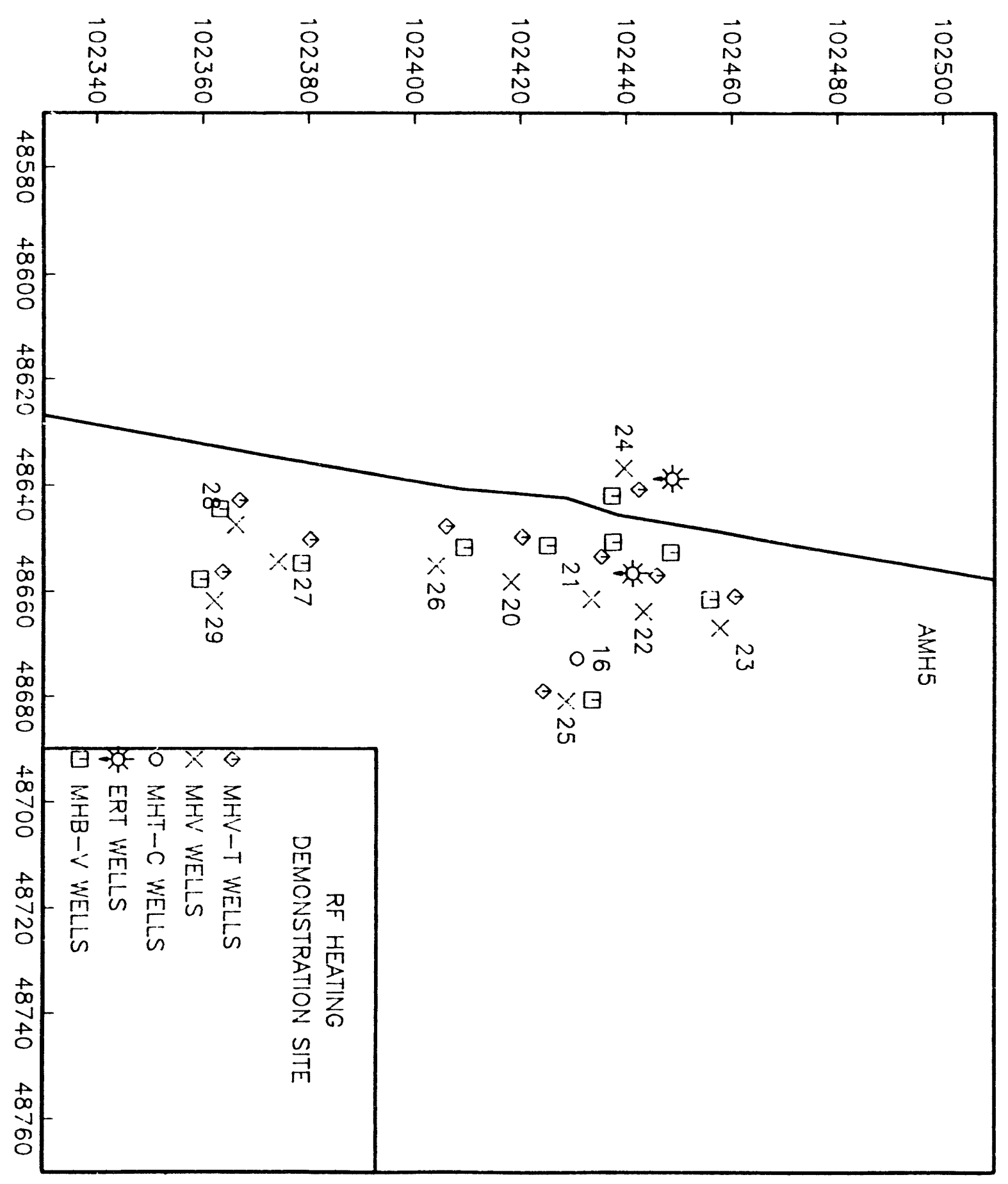




\section{Sampling and Analysis of Sediment Samples}

Sediment samples were collected during characterization activities using the same protocols as the pretest characterization summarized below and described in detail in Eddy et al. (1993), Looney et al. (1993), and Looney et al. (in review).

Sediment samples for VOC analysis and moisture content determination were collected from the split spoon as soon as it was opened using a plunger in a syringe with the end cut off. For moisture content samples, the sediment plug was placed in a preweighed vial that was then sealed. In the laboratory, the vials were weighed, opened, and placed into a drying oven and dried at $60^{\circ} \mathrm{C}$. The samples were reweighed daily until the variance between the individual weighings was small.

Samples for VOC analysis were packed on ice, taken to the laboratory daily, and stored in a refrigerator until analyzed. VOC analyses were performed on a Hewlett-Packard 5890 Gas Chromatograph with electron capture and flame ionization detectors, an HP 19395A Headspace Sampler, an HP 3392A Networking Integrator, and a 60-m $¥ 0.75$-mm inner-diameter Supelco VOCOL wide-bore capillary column coated with a $1.5-\mathrm{mm}$ film. The instrument was calibrated using samples spiked with standard solution. Analytical techniques used are presented in detail in Eddy et al. (1993) and Looney, Eddy, and Sims (in review).

Core specimens for microbial analyses were obtained directly from the split spoon or barrel. Cores were sectioned into 3-in. lengths with sterile spatulas and the outermost layer (about $1 / 4$ the diameter of the core) was scraped off using sterile techniques. The sample was then placed in a sterile Whirl-Pak $\circledast$ bag for immediate transport to the laboratory for analysis.

\section{In Situ Moisture Content}

Moisture content or soil water $\left(\Theta_{g}\right)$ was determined for duplicate samples collected from six sediment coresthree from the pretest characterization (MHV21, MHV22, and MHV24) and three collected directly after the RF source was turned off (MHB21, MHB24, and MHB28)to assess the effects of the heating on the moisture content of the samples (Appendix II).

\section{Methods}

Gravimetric moisture content (g water/ $g$ soil) is the most straight-forward expression of soil water content. Gravimetric moisture is the difference in weight between the dry and moist sediment expressed as a fraction of the moist weight. The most commonly used expression of soil moisture is volumetric moisture content. Since the volumes of each sample was not measured in this study, the volumetric moisture content $\left(\Theta_{v}\right)$ of the sediments can only be estimated. The following assumptions were made in these calculations:

- 1.6 was used for the bulk density of clay

- 1.65 for the bulk density of sand and gravel

- $48 \%$ porosity for the clay fraction

- $35 \%$ for the sand fraction

- 2.6 for the density of clay

- 2.65 for the density of sand and gravel

These numbers are based on geotechnical measurements made on nearby cores from the IDS (Eddy et al. 1993). Grain-size distribution were made by microscopic examination of the cores in the laboratory.

\section{Results}

Calculated values of volumetric moisture content ranged from 36 to $99 \%$ for pretest samples and 34 to $95 \%$ for post-test samples. Three samples had moisture contents of above $100 \%$. These samples were collected from a sandy zones where perched water zones had been observed during drilling and may represent true values.

Two observations can be made about distribution of moislure in the study area. First, in any given core, the clay-rich zones have higher moisture contents than the sandy zones. Second, the pretest moisture content of sediments in the target clay zone zone were substantially reduced during the heating. This is shown in Figure 2; results obtained from pretest boring MHV24 and the adjacent post-test boring MHB24 plotted against depth. In the zone from 33 to $43 \mathrm{ft}$, where the sediments are classified as clays (Appendix II), both the pretest and post-test moisture contents are higher than in the overlying and underlying sandy zones. In the overlying and underlying zones, the moisture contents vary significantly and a clear relationship between pretest and post-test values is not evident. Deviations from the general trends at nearby depths may also result from sampling a different lithology in the pretest and post-test sampling. The same general trends can be observed for MHV21 and MHB21 (Figure 3). The results from the control zone (MHV28) are very similar to the pretest samples. 
Figure 2. Pretest Boring MHV24 and Adjacent Post-test Boring MHB24 Plotted Against Depth

Figure 3. Pretest Boring MHV21 and Adjacent Post-test Boring MHB21 Plotted Against Depth 


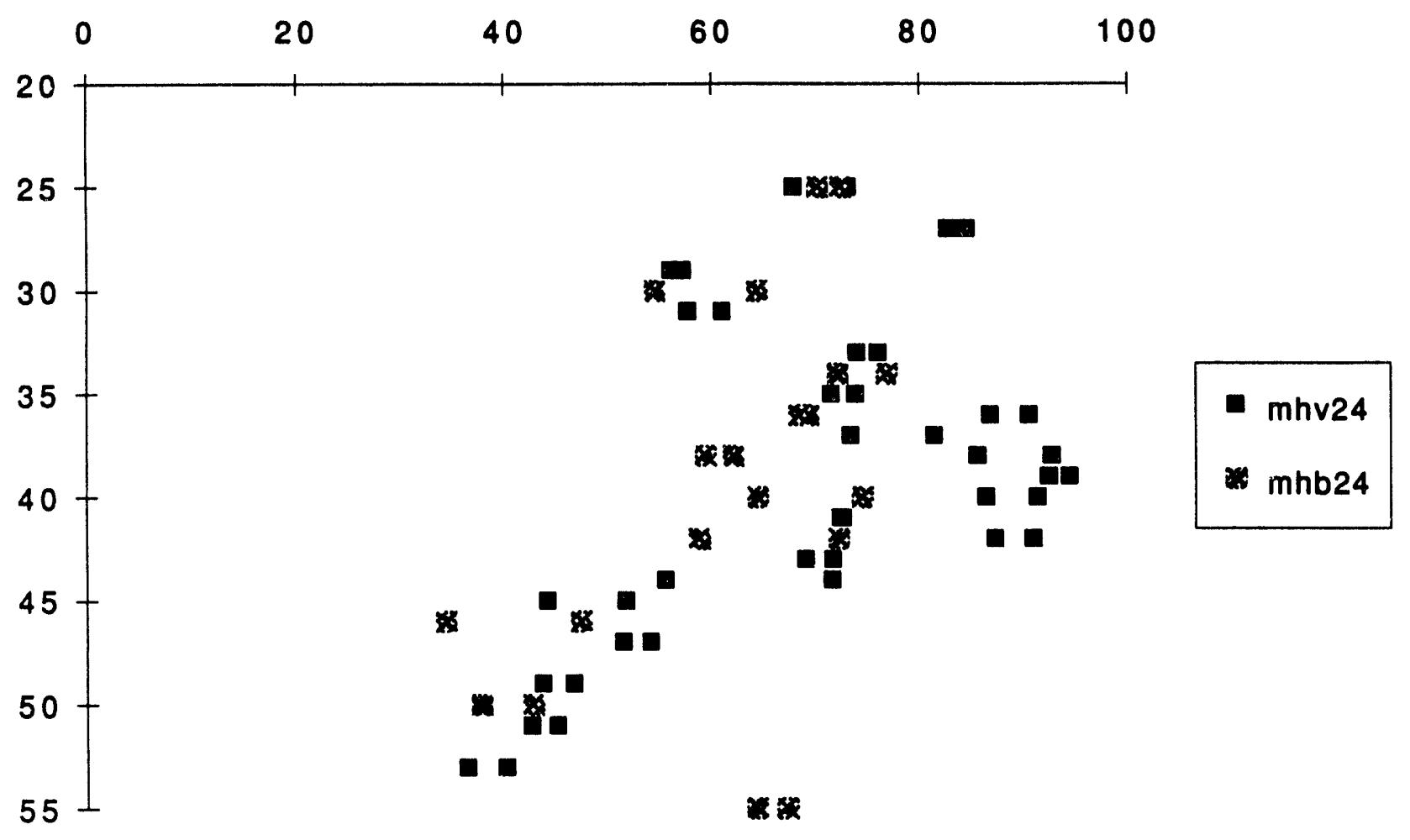




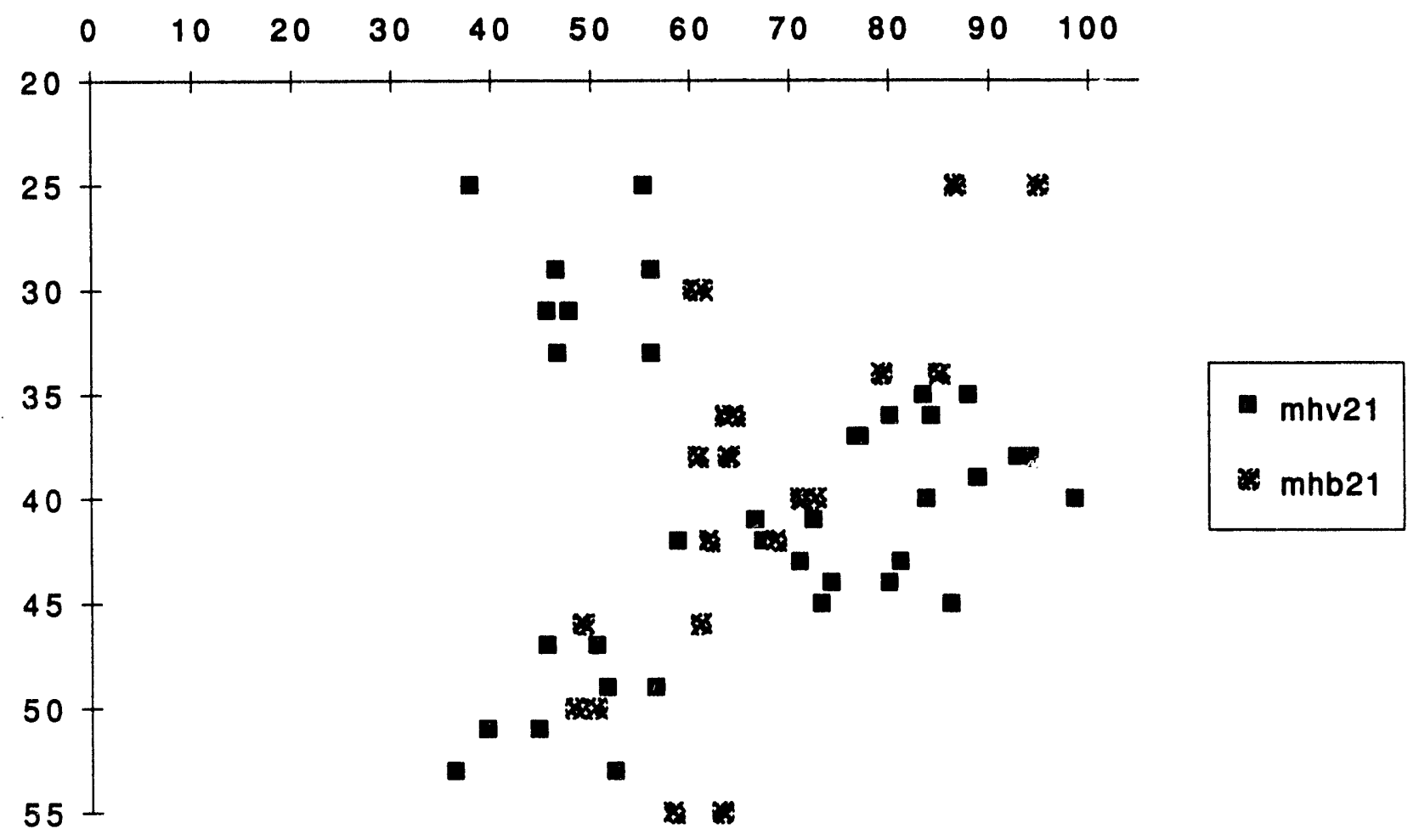




\section{Microbiology}

Sediment samples from MHV21, MHV22T, MHV23, MHV24, MHV25 and MHB21, MHB22, MHB24, and M.HB25 were at 5- $\mathrm{ft}$ intervals (starting at $35 \mathrm{ft}$ ) and evaluated for bacterial and fungal populations prior to and after the RF heating demonstration (Table 4).

\section{Materials and Methods}

Microorganisms were extracted from the core samples by vortexing for 60 seconds with homogenizing buffer $(\mathrm{pH}$ 7.0), sonicated for 30 seconds, and centrifuged at 2200 RPM for five minutes. This process was repeated three times for each sample, and the supernatant pooled after each extraction. Ten $\mu \mathrm{L}$ of extract was placed into each well of three toxoplasmosis slides for acridine orange direct counts (AODCs) and fluorescent antibody staining. Duplicate Biolog GN Microplates were inoculated with the extract for each sample. The plates were incubated at $25^{\circ} \mathrm{C}$. $1 \%$ PTYG plates without the antifungal antibiotic cyclohexamide were inoculated with the spread plate technique by plating 10,100 , and $500 \mu \mathrm{L}$ of the extract in triplicate. One of each was incubated at $25^{\circ}, 45^{\circ}$, and $60^{\circ} \mathrm{C}$. In addition, MHV 23 and MHV24 pretest sediment samples were vortexed in pyrophosphate buffer ( $\mathrm{pH} 7.0$ ), diluted in phosphate buffered saline, and plated in triplicate on $1 \%$ PTYG plates without cyclohexamide. These plates were aiso incubated at the three temperatures. Sediment sample was plated directly on $1 \%$ PTYG plates, spread with $100 \mu \mathrm{L}$ homogenizing buffer, and incubated at all three temperatures.

Selected bacterial strains were analyzed because they represented some of the microbial structural components of the ecosystem being investigated. Species specific direct fluorescent antibodies (DFAs) were prepared against the major microbial communities involved in nitrogen transformation in soils and groundwater (i.e., Azotobacter, Nitrosolobus, Nitrosomonas, and Nitrobacter), as well as those species of Nitrosomonas involved in the production of enzymes capable of degrading TCE; a bacterium that is pathogenic, but widely distributed in nature (Legionella pneumophila); a widely distributed bacterium associated with our systems and involved in iron transformations (Thiobacillus ferrooxidans); and two organisms that have been involved in the destruction of TCE as well as transformation of methane in the laboratory (SRL-MIIF and Methanobacterium). Each of these organisms have been isolated from SRS habitats. The following is a more defined characterization of the bacteria:
- Azotobacter chroococcum is a free-living heterotrophic, nitrogen-fixer that lives under aerobic conditions.

- Nitrosolobus multiformis and Nitrosomonas europaea are morphologically distinct chemolithotrophs that obtain their energy from oxidizing ammonia to nitrite while fixing $\mathrm{CO}_{2}$ as their sole carbon source.

- Nitrobacter agilis and Nitrobacter winogradskyi are chemolithotrophs that secure their energy by oxidizing nitrite to nitrate while fixing $\mathrm{CO}_{2}$ as their sole carbon source.

- SRL-MIIF is a Type II methanotroph isolated from the SRS with TCE-degrading capabilities.

- Methanobacterium formicicum is a heterotrophic, strict anaerobe that produces $\mathrm{CH}_{4}$ from organic compounds such as acetate and cysteine.

- Thiobacillus ferroaxidans grows as a chemolithotroph that gets its energy by oxidizing ferrous iron to ferric iron while fixing $\mathrm{CO}_{2}$ as its carbon source.

- Legionella pneumophila Serogroup 1 is a human pathogen and the major etiologic agent of Legionnaires' Disease, as well as an aquatic and terrestrial bacterium.

\section{Results and Discussion}

Samples collected prior to the RF heating demonstration indicated that after a two-week incubation period, the $1 \%$ PTYG plates incubated at $25^{\circ} \mathrm{C}$ showed very little microbiological growth (Table 4). Highest 1\% PTYG densities for MHV21, MHV22T, and MHV23 were observed at the a depth of $35 \mathrm{ft}$, the most shallow depth sampled from microbiological analyses. Bacterial densities for the pretest collected at MHV24 and MHV25 indicated the greatest bacterial densities were present at 35 and $50 \mathrm{ft}$, . respectively.

Data collected immediately after the RF heating demonstration indicated that viable bacteria from MHB24 grown on $1 \%$ PTYG plates incubated at $25^{\circ} \mathrm{C}$ were below detectable limits. Data collected from MHB21 during the heating phase indicated that the viable plate counts were elevated for samples from 48 and $55 \mathrm{ft}$, which experienced $76^{\circ} \mathrm{F}$ and $70^{\circ} \mathrm{F}$ temperatures, respectively. This represented a significant increase (3-4 orders of magnitude) from the ${ }^{\prime}$ "etest RF heating conditions. Post-test RF heating analyuss indicated that all the samples from MHB22 and MHB25 tested for viable bacteria on 1\% PTYG were below detectable limits, except for the sample at $34 \mathrm{ft}$ from MHB22. None of the samples showed any growth at temperatures of incubation except $25^{\circ} \mathrm{C}$. Fungal components were not a significant portion of the population observed on the 1\% PTYG plates and are thus not reported. 
Direct counts were made using acridine-orange staining techniques and the density of bacteria per gram of dry sediments was calculated. The data are reported in Table 4. The highest direct AODCs for pretest data were observed in MHT22T at all depths (25-55 feet). Direct counts from samples collected from cores MHB21 and MHB24 immediately after heating stopped were elevated. Analysis of samples from the post-RF heating samples showed that densities of bacterial cells remained above the pre-RF heating levels. These data indicate that RF heating appeared to cause an increase in cell density as measured by AODC, but that these bacteria were not readily grown on the $1 \%$ PTYG used for viable counts.

No Biolog GN plates inoculated with the sediment extract showed any positive (purple) wells after two weeks of incubation at $25^{\circ} \mathrm{C}$.

DFA analyses were made for selected bacteria in each of the sediment samples. The organisms were selected in a pragmatic manner in that all of these organisms have been isolated from the IDS and have been characterized during the Integrated Demonstration Project conducted in $\mathbf{M}$ Area. The data provide the direct determination of densitics of specific organisms without having to culture the organisms. The data indicate that most of the nitrogen transforming organisms (i.e., Nitrosolobus multiformis, Nitrosomonas europaea, Nitrobacter agilis, Nitrobacter winogradskyi) demonstrated no appreciable population change over the duration of the RF heating experiment. On the other hand, the densities of Azotobacter chroococcum, the nitrogen-fixing bacterium, were reduced by approximately one order of magnitude.

Methanobacterium formicicum, Thiobacillus ferroaxidans, and SRL-MIIF demonstrated no change over the duration of the heating investigations. A somewhat surprising result was the affect that heating had on the densities of Legionella pneumophila. The data in Table 5 indicate that Legionella pneumophila densities were near the nondetectable limits in the pre-heating phase of the investigation, but increased dramatically (1-2 orders of magnitude) during the heating phase, only to return to preheat levels during the post phase. This is a most surprising result and is somewhat disconcerting. While microorganism respond to a $Q 10$ affect (i.e., a 2-3 fold increase in metabolic activity for each 10 degree rise in temperature until the cardinal temperature is reached), to see this dramatic affect with a pathogen like Legionella has not heretofore been observed. Legionella pneumophila has properties of a thermophilic bacterium that would allow it to survive and grow under temperatures up to $63^{\circ} \mathrm{C}$ in aquatic habitats. The temperatures during the RF heating were above such levels where the bacterium was observed.
Isolation of Legionella pneumophila has been made from M-Area sediments in the past and currently we are attempting to isolate the organism from samples that were heated.

Clayey regions of the subsurface environment are not only a problem from a remediation point of view but also from a microbiological assessment. It has always been difficult to extract and culture microbial populations from the clayrich sediments. This is again confirmed by the high AODC/colony forming unit ratio observed in the RF heating samples. The data indicate that microbial populations are present in the clay regions, but they are difficult to culture; the only way to adequately assess their impact is to use direct probing technologies such as DFAs or gene probes.

\section{Contamination Distribution}

Sediments sampled from the post-test borings were analyzed for both TCE and PCE content. There was a significant increase in the amount and extent of contamination, especially for PCE. Analytical results for all the post-test borings are reported in Appendix III and are plotted against depth in Appendix IV. Three-dimensional imaging techniques were used to prepare pre- and post-test images of TCE and PCE distribution (Figures 4-7).

\section{Methods}

The pre- and post-test values from chemical analysis were entered into a database and three-dimensional representations of the post-test data were prepared using a Silicon Graphics Workstation with Dynamic Graphics Software. The data from the pre- and post-test were modeled using the same parameters. The approach to modeling the data to produce the optimal representation of the data is summarized below and is presented in detail in Eddy and Looney (1993).

Two- and three-dimensional surfaces are used to bound the models to prevent large extrapolation errors in areas where no data control points are present. Second, the effects of important variable parameters (e.g., grid size, grid spacing, and weighting factors) were evaluated (Eddy and Looney 1993). A significant effort was made to use appropriate parameters to calculate the grids for the construction of the three-dimensional models. The grid cell spacing of approximately $5 \times 5 \times 1 \mathrm{ft}$ was chosen to approximate the spacing between sediment borings. A very low $z$-influence factor $(0.001)$ was used to enhance the lateral continuity of between-sample zones to mimic the heterogeneity imposed on the contaminant distribution by the strongly layered sediments. Logarithmic transformations, used 
prior to gridding, are used to preserve the steep gradients found at the edges of the contaminant plumes. A semi-logVolumetric Calculations arithmic scale was chosen for the contour intervals as it provided the best representation given the distribution of data values.

\section{Results}

The pretest distribution of solvent in the study area was strongly heterogeneous, govemed by the location of the contaminant release and migration gradients, and by the interbedded coastal plain sediments (Eddy et al. 1991). Figures 4 and 5 show the pretest distribution of TCE and PCE at concentration of greater than $1 \mathrm{ppm}$ for TCE and PCE. In general, the highest concentrations of TCE and PCE in the study area were found in the clay-rich sediments between 35 and $55 \mathrm{ft}$ that were heated during the remedial demonstration. The pretest images for PCE and TCE show that stratified sediments contribute to the anisotropic distribution of the contamination in the study area. The solvent is distributed in discontinuous lenses that may correspond to clay-rich layers.

In contrast, the post-test models (Figures 6 and 7) also show a reduction in the zone of contamination, and the appearance of the models suggest a more homogeneous distribution of contamination. In addition, preliminary volume calculations indicate the amount of contamination in the modeled area increased during the remedial process. There is more solvent present and the concentrations in the most contaminated zones have increased significantly.

Prelimary conclusions from the modeling suggest that contamiantion was pulled into the model area during and was not removed by vacuum extraction. The appearance of the models indicates that the distribution of fine-grained sediments more strongly controlled the distribution of contamination prior to the heating experiment than after the demonstration. This suggests that the heating and vacuum extraction was successful in mobilizing and concentrating the sediment in the zone of the horizontal well. Unfortunately, because steam formed in the well, the extraction system was not effective at removing the material. These results in conjunction with detailed volumetric estimates will be investigated and discussed in detail in the future report on the remediation system. 
WSRC-RD-93-XXX

Table 4. Microbiological Results from Sediment Samples Evaluated for Bacterial and Fungal Populations

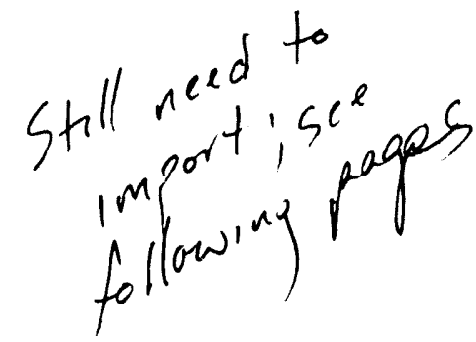


PRE Heating

\begin{tabular}{|c|c|c|c|c|c|c|c|}
\hline Well ID & Depth (ft.) & Date & Temp ${ }^{9} \mathrm{C}$ & \% Molsture & AODC\#/gdw & CFU\#/gdw & $\begin{array}{l}\text { L. pneum/ } \\
\text { gdw }\end{array}$ \\
\hline $\begin{array}{l}\text { MHV.21 } \\
\text { MHV-21 } \\
\text { MHV-21 } \\
\text { MHV-21 } \\
\text { MHV 22T } \\
\text { MHV 22T } \\
\text { MHV 22T } \\
\text { MHV 22T } \\
\text { MHV 22T } \\
\text { MHV 22T } \\
\text { MHV 22T } \\
\text { MHV-23 } \\
\text { MHV-23 } \\
\text { MHV-23 } \\
\text { MHV-23 } \\
\text { MHV-23 } \\
\text { MHV-23 } \\
\text { MHV-23 } \\
\text { MHV-23 } \\
\text { MHV-24 } \\
\text { MHV-24 } \\
\text { MHV-24 } \\
\text { MHV-24 } \\
\text { MHV-25 } \\
\text { MHV-25 } \\
\text { MHV-25 } \\
\text { MHV-25 } \\
\text { MHV-25 }\end{array}$ & $\begin{array}{l}35 \\
40 \\
45 \\
50 \\
25 \\
30 \\
35 \\
40 \\
45 \\
50 \\
55 \\
35 A \\
40 A \\
45 A \\
50 A \\
35 \\
40 \\
45 \\
50 \\
35 \\
40 \\
45 \\
50 \\
35 \\
40 \\
45 \\
50 \\
55\end{array}$ & $\begin{array}{l}11 / 20 / 92 \\
11 / 20 / 92 \\
11 / 20 / 92 \\
11 / 20 / 92 \\
1 / 18 / 93 \\
1 / 18 / 93 \\
1 / 18 / 93 \\
1 / 18 / 93 \\
1 / 18 / 93 \\
1 / 18 / 93 \\
1 / 18 / 93 \\
11 / 20 / 92 \\
11 / 20 / 92 \\
11 / 20 / 92 \\
11 / 20 / 92 \\
11 / 20 / 92 \\
11 / 20 / 92 \\
11 / 20 / 92 \\
11 / 20 / 92 \\
111792 \\
111792 \\
111792 \\
111792 \\
11092 \\
11902 \\
11992 \\
11002 \\
11099\end{array}$ & $\begin{array}{l}\text { PRE } \\
\text { PRE } \\
\text { PRE } \\
\text { PRE } \\
\text { PRE } \\
\text { PRE } \\
\text { PRE } \\
\text { PRE } \\
\text { PRE } \\
\text { PRE } \\
\text { PRE } \\
\text { PRE } \\
\text { PRE } \\
\text { PRE } \\
\text { PRE } \\
\text { PRE } \\
\text { PRE } \\
\text { PRE } \\
\text { PRE } \\
\text { PRE } \\
\text { PRE } \\
\text { PRE } \\
\text { PRE } \\
\text { PRE } \\
\text { PRE } \\
\text { PRE } \\
\text { PRE } \\
\text { PRE }\end{array}$ & $\begin{array}{r}16.86 \\
22.22 \\
15.52 \\
9.23 \\
17.37 \\
11.84 \\
17.89 \\
15.99 \\
11.11 \\
10.79 \\
13.69 \\
11.42 \\
21.50 \\
15.11 \\
8.36 \\
16.86 \\
22.22 \\
15.52 \\
9.23 \\
17.96 \\
20.84 \\
9.27 \\
9.28 \\
13.22 \\
13.78 \\
13.76 \\
6.19 \\
9.58\end{array}$ & $\begin{array}{l}0.00 \mathrm{E}+00 \\
1.14 \mathrm{E}+06 \\
1.97 \mathrm{E}+06 \\
7.81 \mathrm{E}+06 \\
7.90 \mathrm{E}+07 \\
5.36 \mathrm{E}+07 \\
1.57 \mathrm{E}+08 \\
3.29 \mathrm{E}+07 \\
4.44 \mathrm{E}+07 \\
4.63 \mathrm{E}+07 \\
2.14 \mathrm{E}+08 \\
9.60 \mathrm{E}+06 \\
0.00 \mathrm{E}+00 \\
1.10 \mathrm{E}+06 \\
0.00 \mathrm{E}+00 \\
1.29 \mathrm{E}+07 \\
2.09 \mathrm{E}+06 \\
1.06 \mathrm{E}+07 \\
0.00 \mathrm{E}+00 \\
1.29 \mathrm{E}+07 \\
2.09 \mathrm{E}+06 \\
1.06 \mathrm{E}+07 \\
0.00 \mathrm{E}+00 \\
1.29 \mathrm{E}+06 \\
0.00 \mathrm{E}+00 \\
1.14 \mathrm{E}+06 \\
2.40 \mathrm{E}+06 \\
N D\end{array}$ & $\begin{array}{r}1.27 \mathrm{E}+02 \\
<3.70 \mathrm{E}+01 \\
<3.10 \mathrm{E}+01 \\
6.34 \mathrm{E}+01 \\
3.25 \mathrm{E}+04 \\
<6.56+02 \\
<7.11 \mathrm{E}+02 \\
<5.72 \mathrm{E}+02 \\
<5.81 \mathrm{E}+02 \\
<5.35 \mathrm{E}+02 \\
<5.19 \mathrm{E}+02 \\
2.01 \mathrm{E}+03 \\
<8.08 \mathrm{E}+01 \\
<5.85 \mathrm{E}+01 \\
<5.61 \mathrm{E}+01 \\
4.84 \mathrm{E}+02 \\
<6.17 \mathrm{E}+01 \\
<3.44 \mathrm{E}+01 \\
<2.90 \mathrm{E}+01 \\
<1.18 \mathrm{E}+01 \\
8.81 \mathrm{E}+01 \\
<9.94 \mathrm{E}+01 \\
<7.71 \mathrm{E}+01 \\
<4.20 \mathrm{E}+01 \\
<3.32 \mathrm{E}+01 \\
1.49 \mathrm{E}+02 \\
9.76 \mathrm{E}+02 \\
4.84 \mathrm{E}+02\end{array}$ & $\begin{array}{r}<3.69 \mathrm{E}+04 \\
4.24 \mathrm{E}+04 \\
<3.80 \mathrm{E}+04 \\
4.05 \mathrm{E}+04 \\
1.27 \mathrm{E}+05 \\
<7.23 \mathrm{E}+04 \\
<6.14 \mathrm{E}+04 \\
<5.49 \mathrm{E}+04 \\
4.47 \mathrm{E}+05 \\
7.07 \mathrm{E}+04 \\
<3.96 \mathrm{E}+04 \\
<4.16 \mathrm{E}+04 \\
\mathrm{ND}\end{array}$ \\
\hline Well ID & $\begin{array}{l}\text { SRL/ } \\
\text { gdw }\end{array}$ & $\begin{array}{c}\text { Nitros eur.' } \\
\text { grw }\end{array}$ & N. $\underset{g d w}{\operatorname{ggll} / \mathrm{win} /}$ & $\begin{array}{l}\text { Ferrl } \\
\text { gdw }\end{array}$ & $\begin{array}{c}\text { Nitrosolb / } \\
\text { gdw }\end{array}$ & $\begin{array}{c}\text { Azoto/ } \\
\text { gdw }\end{array}$ & $\begin{array}{l}\text { Methano/ } \\
g d w\end{array}$ \\
\hline $\begin{array}{l}\text { MHV-21 } \\
\text { MHV-21 } \\
\text { MHV-21 } \\
\text { MHV-21 } \\
\text { MHV 22T } \\
\text { MHV 22T } \\
\text { MHV 22T } \\
\text { MHV 22T } \\
\text { MHV 22T } \\
\text { MHV 22T } \\
\text { MHV 22T } \\
\text { MHV-23 } \\
\text { MHV-23 } \\
\text { MHV-23 } \\
\text { MHV-23 }\end{array}$ & $\begin{array}{l}4.51 E+04 \\
3.94 E+04 \\
3.03 E+06 \\
3.38 E+04 \\
2.00 E+04 \\
2.08 E+04 \\
2.18 E+04 \\
1.95 E+04 \\
9.79 E+04 \\
2.18 E+04 \\
2.08 E+04\end{array}$ & $\begin{array}{r}4.51 \mathrm{E}+04 \\
3.94 \mathrm{E}+04 \\
<3.40 \mathrm{E}+04 \\
<3.38 \mathrm{E}+04 \\
6.20 \mathrm{E}+05 \\
<2.08 \mathrm{E}+04 \\
<2.18 \mathrm{E}+04 \\
<1.95 \mathrm{E}+04 \\
<1.96 \mathrm{E}+04 \\
<2.18 \mathrm{E}+04 \\
<2.08 \mathrm{E}+04\end{array}$ & $\begin{array}{r}4.51 E+04 \\
3.94 E+04 \\
<3.40 E+04 \\
<3.38 E+04 \\
<2.00 E+04 \\
8.54 E+05 \\
<2.18 E+04 \\
<1.95 E+04 \\
<1.95 E+04 \\
<2.17 E+04 \\
<2.08 E+04\end{array}$ & $\begin{array}{r}4.51 \mathrm{E}+04 \\
3.94 \mathrm{E}+04 \\
<3.40 \mathrm{E}+04 \\
<3.37 \mathrm{E}+04 \\
<2.00 \mathrm{E}+04 \\
<2.08 \mathrm{E}+04 \\
<2.18 \mathrm{E}+04 \\
<1.95 \mathrm{E}+04 \\
<1.95 \mathrm{E}+04 \\
<2.18 \mathrm{E}+04 \\
<2.08 \mathrm{E}+04\end{array}$ & $\begin{array}{r}4.51 E+04 \\
3.94 E+04 \\
<3.40 E+04 \\
<3.37 E+04 \\
<2.00 E+04 \\
<2.08 E+04 \\
<2.18 E+04 \\
<1.94 E+04 \\
<1.95 E+04 \\
<2.17 E+04 \\
<2.08 E+04\end{array}$ & $\begin{array}{r}4.51 \mathrm{E}+04 \\
3.94 \mathrm{E}+04 \\
<3.40 \mathrm{E}+04 \\
<3.37 \mathrm{E}+04 \\
<2.00 \mathrm{E}+04 \\
1.33 \mathrm{E}+06 \\
1.99 \mathrm{E}+06 \\
<1.94 \mathrm{E}+04 \\
<1.95 \mathrm{E}+04 \\
2.61 \mathrm{E}+05 \\
2.08 \mathrm{E}+05\end{array}$ & $\begin{array}{r}4.51 E+04 \\
3.94 E+04 \\
<3.40 E+04 \\
<3.37 E+04 \\
<2.00 E+04 \\
<2.08 E+04 \\
8.73 E+04 \\
<1.94 E+04 \\
<1.95 E+04 \\
<2.18 E+04 \\
<2.08 E+04\end{array}$ \\
\hline $\begin{array}{l}\text { MHV-23 } \\
\text { MHV.22 } \\
\text { MHV-23 } \\
\text { MHV-23 } \\
\text { MHV-24 } \\
\text { MHV-24 } \\
\text { MHV-24 } \\
\text { MHV-24 } \\
\text { MHV.25 } \\
\text { MHV.25 } \\
\text { MHV.25 } \\
\text { MHV-25 } \\
\text { MHV.25 }\end{array}$ & $\begin{array}{c}3.69 E+04 \\
1.27 E+05 \\
3.80 E+04 \\
4.05 E+04 \\
6.37 E+04 \\
6.51 E+05 \\
6.14 E+04 \\
5.48 E+04 \\
7.34 E+06 \\
5.76 E+06 \\
3.96 E+04 \\
1.25 E+05 \\
N D\end{array}$ & $\begin{array}{r}<3.70 \mathrm{E}+04 \\
4.24 \mathrm{E}+04 \\
<3.80 \mathrm{E}+04 \\
4.05 \mathrm{E}+04 \\
6.37 \mathrm{E}+04 \\
1.45 \mathrm{E}+05 \\
6.14 \mathrm{E}+04 \\
5.48 \mathrm{E}+04 \\
4.47 \mathrm{E}+04 \\
<3.53 \mathrm{E}+04 \\
3.96 \mathrm{E}+04 \\
4.16 \mathrm{E}+04 \\
\mathrm{ND}\end{array}$ & $\begin{array}{r}<3.69 \mathrm{E}+04 \\
2.54 \mathrm{E}+05 \\
<3.79 \mathrm{E}+04 \\
4.05 \mathrm{E}+04 \\
6.37 \mathrm{E}+04 \\
7.23 \mathrm{E}+04 \\
6.14 \mathrm{E}+04 \\
5.48 \mathrm{E}+04 \\
4.47 \mathrm{E}+04 \\
7.07 \mathrm{E}+04 \\
1.19 \mathrm{E}+05 \\
4.16 \mathrm{E}+04 \\
N D\end{array}$ & $\begin{array}{r}<3.69 E+04 \\
4.24 E+04 \\
<3.79 E+04 \\
4.05 E+04 \\
6.37 E+04 \\
2.17 E+05 \\
6.14 E+04 \\
5.48 E+04 \\
2.24 E+05 \\
7.07 E+04 \\
7.92 E+04 \\
4.16 E+04 \\
N D\end{array}$ & $\begin{array}{r}<3.69 \mathrm{E}+04 \\
4.24 \mathrm{E}+04 \\
<3.79 \mathrm{E}+04 \\
4.05 \mathrm{E}+04 \\
6.37 \mathrm{E}+04 \\
7.23 \mathrm{E}+04 \\
6.14 \mathrm{E}+04 \\
1.64 \mathrm{E}+05 \\
4.47 \mathrm{E}+04 \\
<3.53 \mathrm{E}+04 \\
3.96 \mathrm{E}+04 \\
4.16 \mathrm{E}+04 \\
\mathrm{ND}\end{array}$ & $\begin{array}{r}<3.69 \mathrm{E}+04 \\
5.26 \mathrm{E}+06 \\
<3.79 \mathrm{E}+04 \\
4.05 \mathrm{E}+04 \\
2.55 \mathrm{E}+05 \\
1.45 \mathrm{E}+05 \\
6.14 \mathrm{E}+04 \\
5.48 \mathrm{E}+04 \\
4.30 \mathrm{E}+06 \\
1.06 \mathrm{E}+05 \\
7.92 \mathrm{E}+04 \\
4.16 \mathrm{E}+04 \\
\mathrm{ND}\end{array}$ & $\begin{array}{r}4.47 \mathrm{E}+04 \\
<3.53 \mathrm{E}+04 \\
3.96 \mathrm{E}+04 \\
4.16 \mathrm{E}+04 \\
6.37 \mathrm{E}+04 \\
1.45 \mathrm{E}+05 \\
6.14 \mathrm{E}+04 \\
5.48 \mathrm{E}+04 \\
4.47 \mathrm{E}+04 \\
<3.53 \mathrm{E}+04 \\
3.96 \mathrm{E}+04 \\
4.16 \mathrm{E}+04 \\
\mathrm{ND}\end{array}$ \\
\hline
\end{tabular}


During Heating

\begin{tabular}{|c|c|c|c|c|c|c|c|}
\hline Well ID & Depth (ft.) & Date & Temp ${ }^{\circ} \mathrm{C}$ & \% Molsture & AODC\#/gdw & CFU\#/gdw & $\begin{array}{l}\text { L. pneum } \\
/ \mathrm{gdw}\end{array}$ \\
\hline MHB 21 & 25 & $4 / 29 / 93$ & *68 & 11.36 & $5.61 E+07$ & $<6.12 \mathrm{E}+01$ & $1.68 E+06$ \\
\hline MHB 21 & 30 & $4 / 29 / 93$ & $\bullet 73$ & 12.05 & $6.28 \mathrm{E}+07$ & $<5.35 \mathrm{E}+01$ & $8.84 E+05$ \\
\hline MHB 21 & 34 & $4 / 29 / 93$ & $* 83$ & 13.00 & $5.56 E+07$ & $<5.14 \mathrm{E}+01$ & $8.75 E+05$ \\
\hline MHB 21 & 38 & $4 / 29 / 93$ & 88 & 18.06 & $1.12 \mathrm{E}+08$ & $<5.82 \mathrm{E}+01$ & $8.86 E+05$ \\
\hline MHB 21 & 42 & $4 / 29 / 93$ & $* 84$ & 19.95 & $1.94 \mathrm{E}+08$ & $<6.32 \mathrm{E}+01$ & $2.69 E+05$ \\
\hline MHB 21 & 48 & $4 / 29 / 93$ & 76 & 11.15 & $1.08 E+08$ & $1.80 \mathrm{E}+04$ & $5.91 E+05$ \\
\hline MHB 21 & 55 & $4 / 29 / 93$ & $* 70$ & 12.59 & $1.49 E+08$ & $9.91 E+04$ & $6.12 \mathrm{E}+05$ \\
\hline MHB-24 & 25 & $4 / 28 / 93$ & $* 70$ & 13.36 & $1.05 E+08$ & $<5.09 \mathrm{E}+01$ & $7.44 \mathrm{E}+04$ \\
\hline MHB-24 & 30 & $4 / 28 / 93$ & $* 85$ & 32.07 & $6.26 \bar{E}+07$ & $<5.66 \mathrm{E}+01$ & $3.19 \mathrm{E}+06$ \\
\hline MHB-24 & 34 & $4 / 28 / 93$ & $\bullet 120$ & 19.03 & $3.93 E+07$ & $<5.17 E+01$ & $1.72 E+06$ \\
\hline MHB-24 & 38 & $4 / 28 / 93$ & 145 & 19.03 & $7.49 E+07$ & $<5.10 \mathrm{E}+01$ & $1.72 E+06$ \\
\hline MHB-24 & 42 & $4 / 28 / 93$ & $* 120$ & 18.32 & $3.24 \mathrm{E}+07$ & $<5.74 \mathrm{E}+01$ & $4.43 \mathrm{E}+06$ \\
\hline MHB-24 & 48 & $4 / 28 / 93$ & 86 & 10.67 & $3.24 \mathrm{E}+07$ & $<5.02 E+01$ & $3.50 \mathrm{E}+06$ \\
\hline MHB-24 & 55 & $4 / 28 / 93$ & $* 70$ & 9.08 & $3.56 \mathrm{E}+07$ & $<4.90 \mathrm{E}+01$ & $3.09 E+06$ \\
\hline Well ID & $\begin{array}{l}\text { SRL } \\
\text { gdw }\end{array}$ & $\begin{array}{c}\text { Nitros eur/ } \\
\text { grw }\end{array}$ & $\begin{array}{l}\text { N. agll/win/ } \\
\text { gdw }\end{array}$ & $\begin{array}{l}\text { Ferr/ } \\
\text { gdv }\end{array}$ & $\begin{array}{c}\text { Nitrosolb / } \\
\text { gdw }\end{array}$ & $\begin{array}{l}\text { Azoto/ } \\
\text { gdw }\end{array}$ & $\begin{array}{c}\text { Methano/ } \\
\text { gdw }\end{array}$ \\
\hline MHB 21 & $1.55 \mathrm{E}+04$ & $<1.55 \mathrm{E}+04$ & $<1.55 E+04$ & $<1.55 \mathrm{E}+04$ & $<1.55 \mathrm{E}+04$ & $<1.55 \mathrm{E}+04$ & $<1.55 E+04$ \\
\hline MHB 21 & $1.40 \mathrm{E}+04$ & $<1.40 E+04$ & $<1.40 \mathrm{E}+04$ & $<1.40 E+04$ & $<1.40 E+04$ & $<1.40 E+04$ & $<1.40 \mathrm{E}+04$ \\
\hline MHB 21 & $1.23 E+04$ & $<1.23 E+04$ & $<1.23 E+04$ & $<1.23 E+04$ & $<1.23 E+04$ & $<1.23 E+04$ & $<1.23 \mathrm{E}+04$ \\
\hline MHB 21 & $1.45 \mathrm{E}+04$ & $<1.45 E+04$ & $<1.45 E+04$ & $<1.13 E+04$ & $<1.45 E+04$ & $<1.45 \mathrm{E}+04$ & $<1.45 \mathrm{E}+04$ \\
\hline MHB 21 & $1.49 E+05$ & $<1.49 E+04$ & $<1.49 E+04$ & $<1.49 E+04$ & $<1.49 \mathrm{E}+04$ & $4.48 E+04$ & $<1.49 \mathrm{E}+04$ \\
\hline MHB 21 & $1.64 E+04$ & $<1.64 E+04$ & $<1.64 \mathrm{E}+04$ & $<1.64 E+04$ & $<1.64 E+04$ & $1.64 \mathrm{E}+04$ & $<1.64 \mathrm{E}+04$ \\
\hline MHB 21 & $1.70 \mathrm{E}+04$ & $<1.70 E+04$ & $<1.70 E+04$ & $<1.70 E+04$ & $<1.70 E+04$ & $<1.70 E_{-04}$ & $<1.70 \mathrm{E}+04$ \\
\hline MHB-24 & $2.68 E+05$ & $<1.49 E+04$ & $<1.48 E+04$ & $<1.48 E+04$ & $<1.48 E+04$ & $<1.48 E+04$ & $<1.48 \mathrm{E}+04$ \\
\hline МHB-24 & $1.68 \mathrm{E}+05$ & $<2.09 E+04$ & $<2.09 E+04$ & $<2.09 E+04$ & $<2.09 E+04$ & $<2.09 E+04$ & $<2.09 E+04$ \\
\hline MHB-24 & $1.64 \mathrm{E}+05$ & $<1.49 \mathrm{E}+04$ & $<1.49 E+04$ & $<1.49 E+04$ & $5.98 E+04$ & $<1.49 E+04$ & $<1.49 \mathrm{E}+04$ \\
\hline MHB-24 & $1.64 \mathrm{E}+05$ & $<1.49 E+04$ & $<1.49 \mathrm{E}+04$ & $<1.49 \mathrm{E}+04$ & $5.98 E+04$ & $<1.49 E+04$ & $<1.49 \mathrm{E}+04$ \\
\hline MHB-24 & $2.69 E+04$ & $<1.34 \mathrm{E}+04$ & $<1.34 E+04$ & $<1.34 \mathrm{E}+04$ & $<1.34 E+04$ & $<1.34 \mathrm{E}+04$ & $<1.34 \mathrm{E}+04$ \\
\hline MHB-24 & $1.56 \mathrm{E}+04$ & $<1.56 \mathrm{E}+04$ & $<3.11 E+04$ & $<1.56 \mathrm{E}+04$ & $<1.55 E+04$ & $<1.55 \mathrm{E}+04$ & $<1.55 E+04$ \\
\hline MHB-24 & $1.32 E+04$ & $<1.32 E+04$ & $<1.32 \mathrm{E}+04$ & $<1.31 \mathrm{E}+04$ & $<1.31 \mathrm{E}+04$ & $<1.31 E+04$ & $<1.31 \mathrm{E}+04$ \\
\hline
\end{tabular}




\section{Post Heating}

\begin{tabular}{|c|c|c|c|c|c|c|c|}
\hline Well ID & Depth (ft) & Date & Temp ${ }^{2} \mathrm{C}$ & \% Molsture & AODC $* / g d w$ & CFU\#/gdw & $\begin{array}{l}\text { L. pneum/ } \\
\text { gdw }\end{array}$ \\
\hline $\begin{array}{l}\text { MHB-25 } \\
\text { MHB-25 } \\
\text { MHB-25 } \\
\text { MHB-25 } \\
\text { MHB-25 } \\
\text { MHB-25 } \\
\text { MHB-25 } \\
\text { MHB-22 } \\
\text { MHB-22 } \\
\text { MHB-22 } \\
\text { MHB-22 } \\
\text { MHB-22 } \\
\text { MHB-22 } \\
\text { MHB-22 }\end{array}$ & $\begin{array}{l}24-26 \\
30-32 \\
34-36 \\
38-40 \\
42-44 \\
48-50 \\
54-56 \\
25 \\
30 \\
34 \\
38 \\
42 \\
48 \\
55\end{array}$ & $\begin{array}{l}6 / 2 / 92 \\
6 / 2 / 92 \\
6 / 2 / 92 \\
6 / 2 / 92 \\
6 / 2 / 92 \\
6 / 2 / 92 \\
6 / 2 / 92 \\
6 / 3 / 93 \\
6 / 3 / 93 \\
6 / 3 / 93 \\
6 / 3 / 93 \\
6 / 3 / 93 \\
6 / 3 / 93 \\
6 / 3 / 93\end{array}$ & $\begin{array}{l}\text { POST } \\
\text { POST } \\
\text { POST } \\
\text { POST } \\
\text { POST } \\
\text { POST } \\
\text { POST } \\
\text { POST } \\
\text { POST } \\
\text { POST } \\
\text { POST } \\
\text { POST } \\
\text { POST } \\
\text { POST }\end{array}$ & $\begin{array}{l}12.62 \\
11.66 \\
19.10 \\
20.19 \\
20.92 \\
10.01 \\
10.97 \\
12.01 \\
10.60 \\
10.22 \\
18.74 \\
18.71 \\
11.66 \\
15.28\end{array}$ & $\begin{array}{l}3.64 E+07 \\
1.13 E+08 \\
1.07 E+08 \\
2.39 E+07 \\
1.61 E+08 \\
3.74 E+07 \\
6.20 E+07 \\
1.49 E+08 \\
3.28 E+08 \\
1.07 E+07 \\
2.10 E+08 \\
6.13 E+07 \\
8.78 E+07 \\
1.97 E+08\end{array}$ & $\begin{array}{r}<6.54 \mathrm{E}+01 \\
<6.50 \mathrm{E}+01 \\
<7.40 \mathrm{E}+01 \\
<4.34 \mathrm{E}+01 \\
<4.45 \mathrm{E}+01 \\
<3.93 \mathrm{E}+01 \\
<4.66 \mathrm{E}+01 \\
<3.79 \mathrm{E}+01 \\
<3.85 \mathrm{E}+01 \\
1.84 \mathrm{E}+04 \\
<7.80 \mathrm{E}+01 \\
<5.65 \mathrm{E}+01 \\
<4.89 \mathrm{E}+01 \\
<6.56 \mathrm{E}+01\end{array}$ & $\begin{array}{r}<2.43 \mathrm{E}+04 \\
1.89 \mathrm{E}+05 \\
<2.57 \mathrm{E}+04 \\
7.94 \mathrm{E}+04 \\
1.49 \mathrm{E}+05 \\
1.28 \mathrm{E}+05 \\
<1.57 \mathrm{E}+04 \\
8.92 \mathrm{E}+04 \\
2.30 \mathrm{E}+05 \\
2.50 \mathrm{E}+05 \\
<2.77 \mathrm{E}+04 \\
<3.35 \mathrm{E}+04 \\
<1.30 \mathrm{E}+04 \\
5.05 \mathrm{E}+04\end{array}$ \\
\hline Well ID & $\begin{array}{l}\text { SRL/ } \\
\text { gdw }\end{array}$ & $\begin{array}{c}\text { Nitros eur/ } \\
\text { grw }\end{array}$ & $\begin{array}{l}\text { N. } \operatorname{agl} 1 / \mathrm{win} / \\
\mathrm{gdw}\end{array}$ & $\begin{array}{l}\text { Ferrl } \\
\text { sdw }\end{array}$ & $\begin{array}{c}\text { Nitrosolb / } \\
\text { gdw }\end{array}$ & $\begin{array}{c}\text { Azotol } \\
\text { gdw }\end{array}$ & $\begin{array}{c}\text { Methano/ } \\
\text { gdw }\end{array}$ \\
\hline $\begin{array}{l}\text { MHB-25 } \\
\text { MHB-25 } \\
\text { MHB-25 } \\
\text { MHB-25 } \\
\text { MHB-25 } \\
\text { MHB-25 } \\
\text { MHB-25 } \\
\text { MHB-22 } \\
\text { MHB-22 } \\
\text { MHB-22 } \\
\text { MHB-22 } \\
\text { MHB-22 } \\
\text { MHB-22 } \\
\text { MHB-22 }\end{array}$ & $\begin{array}{l}2.42 E+04 \\
2.36 E+04 \\
2.57 E+04 \\
1.59 E+04 \\
1.94 E+06 \\
1.28 E+04 \\
1.57 E+04 \\
2.23 E+04 \\
6.91 E+04 \\
2.28 E+04 \\
2.77 E+04 \\
1.12 E+04 \\
1.30 E+04 \\
1.69 E+04\end{array}$ & $\begin{array}{r}<2.42 E+04 \\
<2.36 E+04 \\
<2.57 E+04 \\
<1.59 E+04 \\
<1.86 E+04 \\
<1.28 E+04 \\
<1.57 E+04 \\
6.69 E+04 \\
<2.30 E+04 \\
<2.27 E+04 \\
<2.77 E+04 \\
<1.12 E+04 \\
<1.30 E+04 \\
<1.69 E+04\end{array}$ & $\begin{array}{l}<2.42 \mathrm{E}+04 \\
<2.36 \mathrm{E}+04 \\
<2.56 \mathrm{E}+04 \\
<1.58 \mathrm{E}+04 \\
<1.86 \mathrm{E}+04 \\
<1.28 \mathrm{E}+04 \\
<1.56 \mathrm{E}+04 \\
<2.23 \mathrm{E}+04 \\
<2.30 \mathrm{E}+04 \\
<2.27 \mathrm{E}+04 \\
<2.76 \mathrm{E}+04 \\
<1.12 \mathrm{E}+04 \\
<1.30 \mathrm{E}+04 \\
<1.69 \mathrm{E}+04\end{array}$ & $\begin{array}{r}<2.42 E+04 \\
<2.36 E+04 \\
7.70 E+04 \\
<1.58 E+04 \\
<1.86 E+04 \\
<1.28 E+04 \\
<1.56 E+04 \\
<2.23 E+04 \\
<2.30 E+04 \\
<2.27 E+04 \\
<2.76 E+04 \\
<1.11 E+04 \\
<1.30 E+04 \\
<1.68 E+04\end{array}$ & $\begin{array}{l}<2.42 \mathrm{E}+04 \\
<2.36 \mathrm{E}+04 \\
<2.56 \mathrm{E}+04 \\
<1.58 \mathrm{E}+04 \\
<1.86 \mathrm{E}+04 \\
<1.28 \mathrm{E}+04 \\
<1.56 \mathrm{E}+04 \\
<2.23 \mathrm{E}+04 \\
<2.30 \mathrm{E}+04 \\
<2.27 \mathrm{E}+04 \\
<2.76 \mathrm{E}+04 \\
<1.11 \mathrm{E}+04 \\
<1.30 \mathrm{E}+04 \\
<1.68 \mathrm{E}+04\end{array}$ & $\begin{array}{r}<2.42 \mathrm{E}+04 \\
<2.36 \mathrm{E}+04 \\
<2.56 \mathrm{E}+04 \\
<1.58 \mathrm{E}+04 \\
<1.86 \mathrm{E}+04 \\
<1.28 \mathrm{E}+04 \\
<1.56 \mathrm{E}+04 \\
2.23 \mathrm{E}+04 \\
3.91 \mathrm{E}+05 \\
<2.27 \mathrm{E}+04 \\
<2.76 \mathrm{E}+04 \\
<1.11 \mathrm{E}+04 \\
<1.30 \mathrm{E}+04 \\
<1.68 \mathrm{E}+04\end{array}$ & $\begin{array}{l}<2.42 \mathrm{E}+04 \\
<2.36 \mathrm{E}+04 \\
<2.56 \mathrm{E}+04 \\
<1.58 \mathrm{E}+04 \\
<1.86 \mathrm{E}+04 \\
<1.28 \mathrm{E}+04 \\
<1.56 \mathrm{E}+04 \\
<2.23 \mathrm{E}+04 \\
<2.30 \mathrm{E}+04 \\
<2.27 \mathrm{E}+04 \\
<2.76 \mathrm{E}+04 \\
<1.11 \mathrm{E}+04 \\
<1.30 \mathrm{E}+04 \\
<1.68 \mathrm{E}+04\end{array}$ \\
\hline
\end{tabular}


Figure 4. Concentration of Trichloroethylene Prior to the Radio Frequency Heating Demonstration 
Figure 5. Concentration of Tetrachloroethylene Prior to the Radio Frequency Heating Demonstration 


\section{RF Heating / Pretest Distribution of TCE}

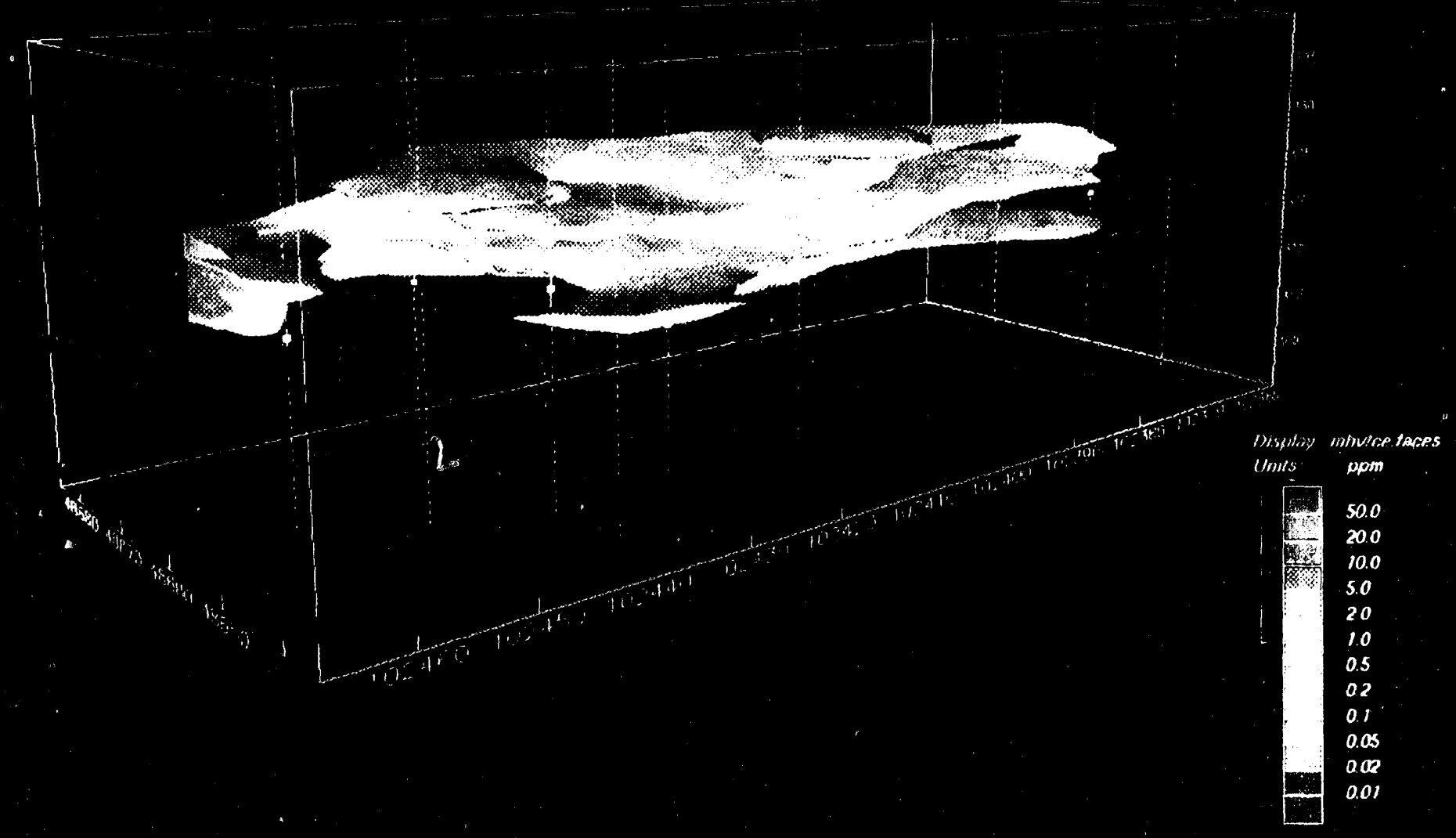




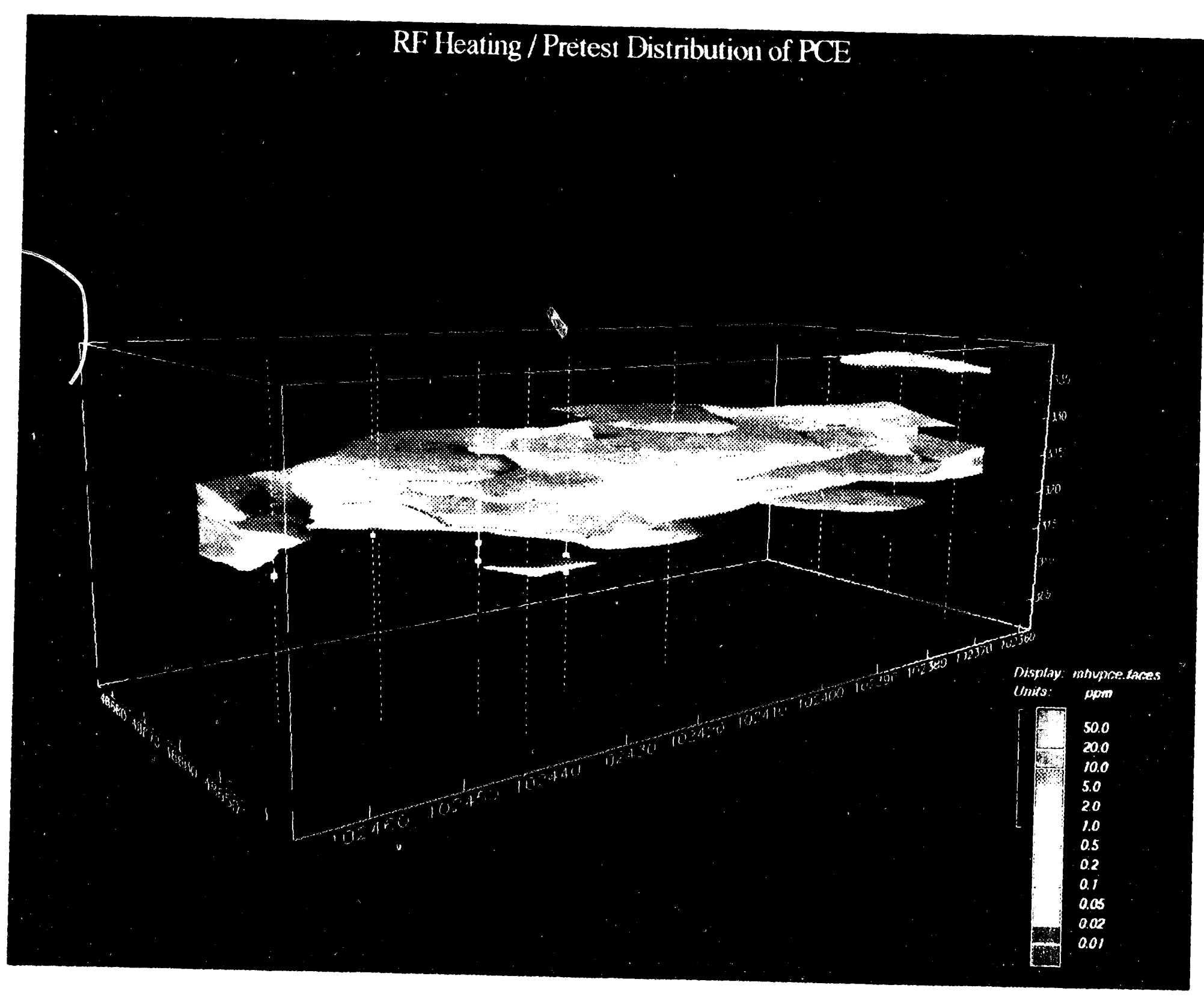


Radio Frequency Heating Demonstration Site Characterization

Figure 6. Concentration of Trichloroethylene After the Radio Frequency Heating Demonstration 
Figure 7. Concentration of Tetrachloroethylene After the Radio Frequency Heating Demonstration 


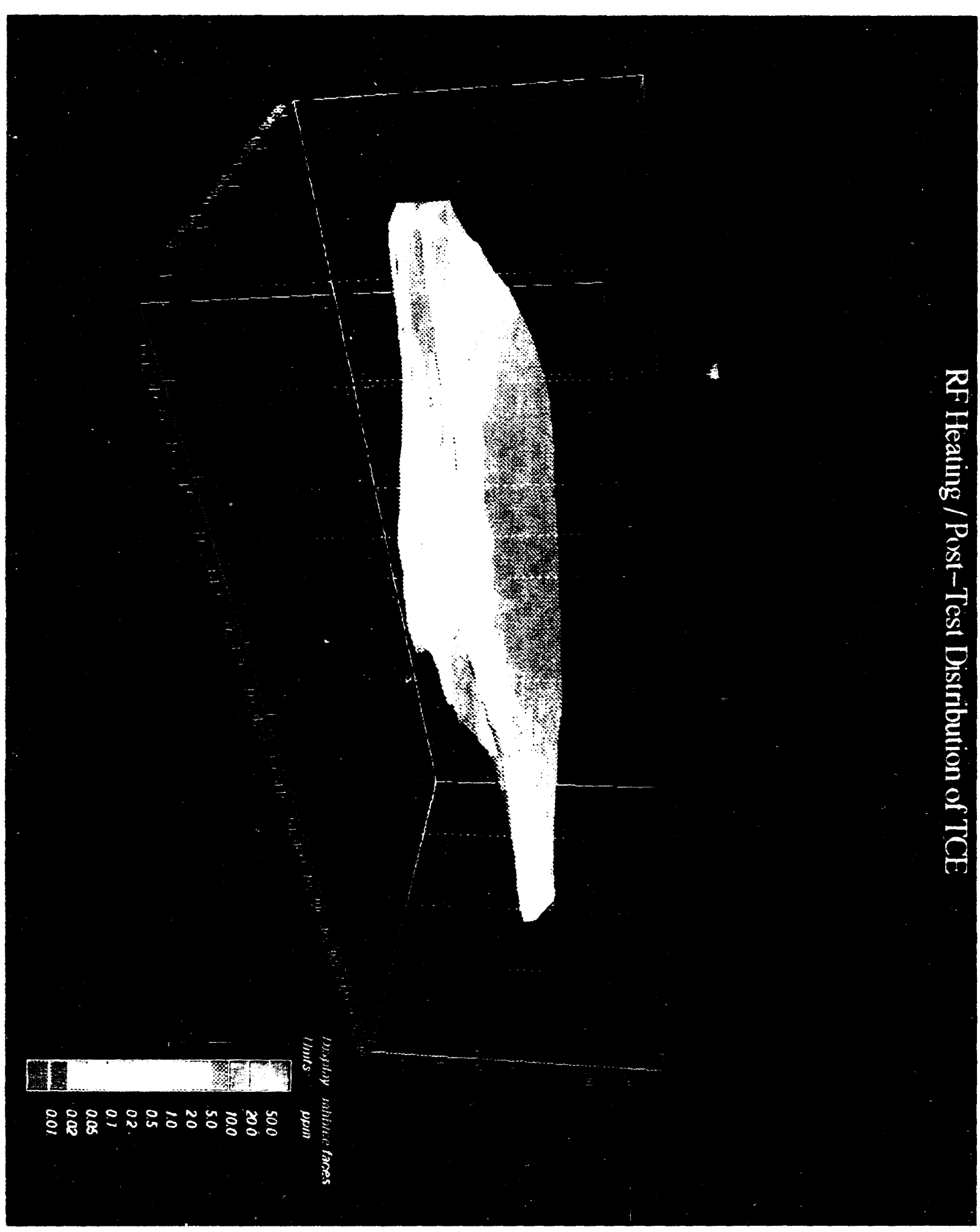




\section{RF Heating / Post-Test Distribution of PCE}

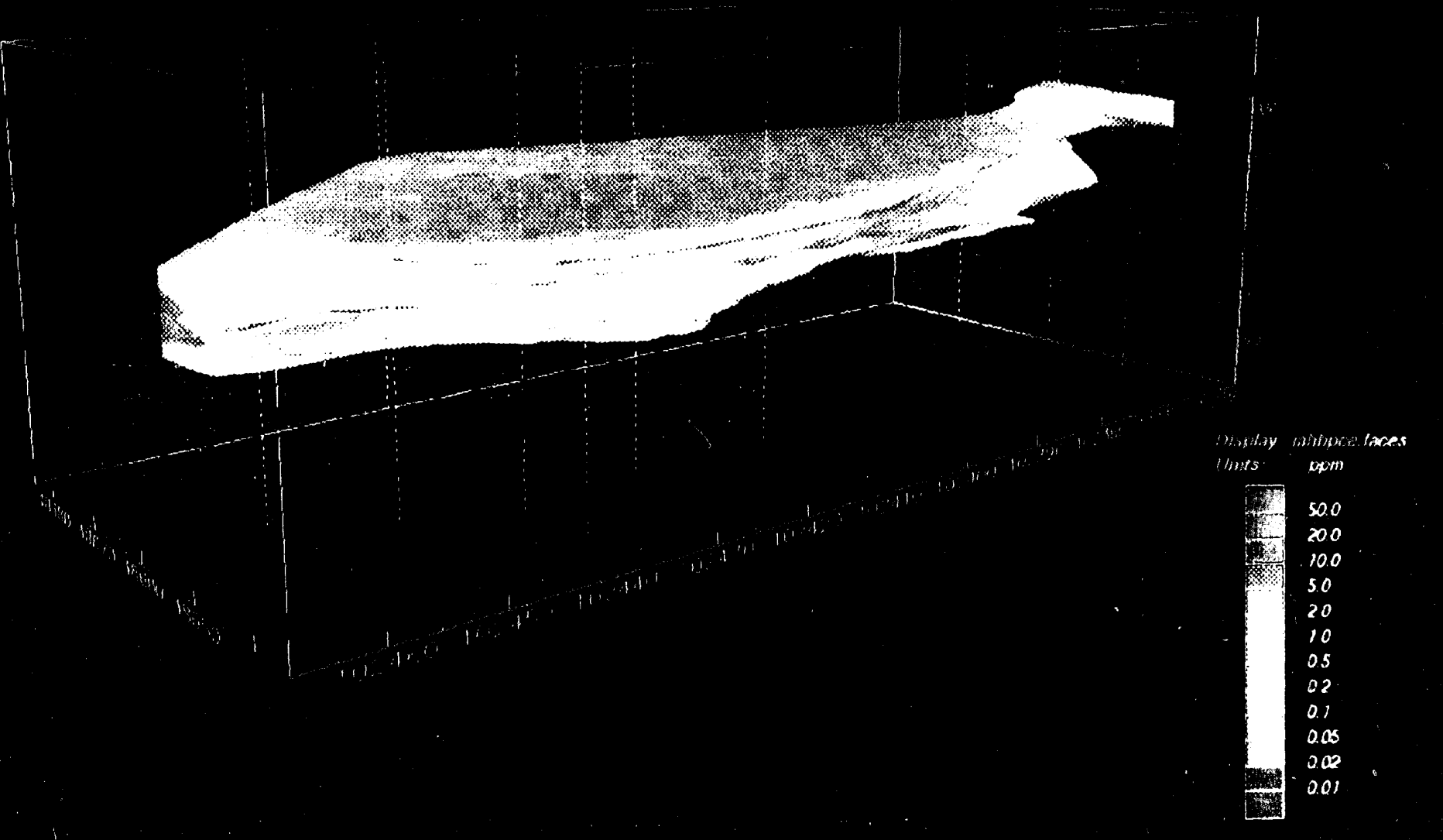




\section{References}

Eddy, C. A., B. B. Looney, J. M. Dougherty, T. C. Hazen, and D. S. Kaback, 1991, Characterization of the Geology, Geochemistry, Hydrology and Microbiology of the In-Situ Air Stripping Demonstration Site at the Savannah River Site, USDOE Report WSRC-RD-91-21, Westinghouse Savannah River Co., Savannah River Laboratory, Aiken, SC 29808, 118p.

The information in this report was developed during the course of work under Contract No. DE-AC09-89SR18035 with the U.S. Department of Energy. 


\section{Appendix 1-Core Descriptions}

All core descriptions follow the format given in ESSOP-2-15: Microscopic Examination Of Sediment Cores. 


\section{MONITORING WELL CONSTRUCTION DIAGRAM}

DRILLING SUBCONTRACTOR EMTC

DAILLER Blake Cabit

DATE OF WELL INSTALLATION $11 / 16 / 92$

TECH. O.S.ICO.NAME Bill Joyce/SEC Dunohue

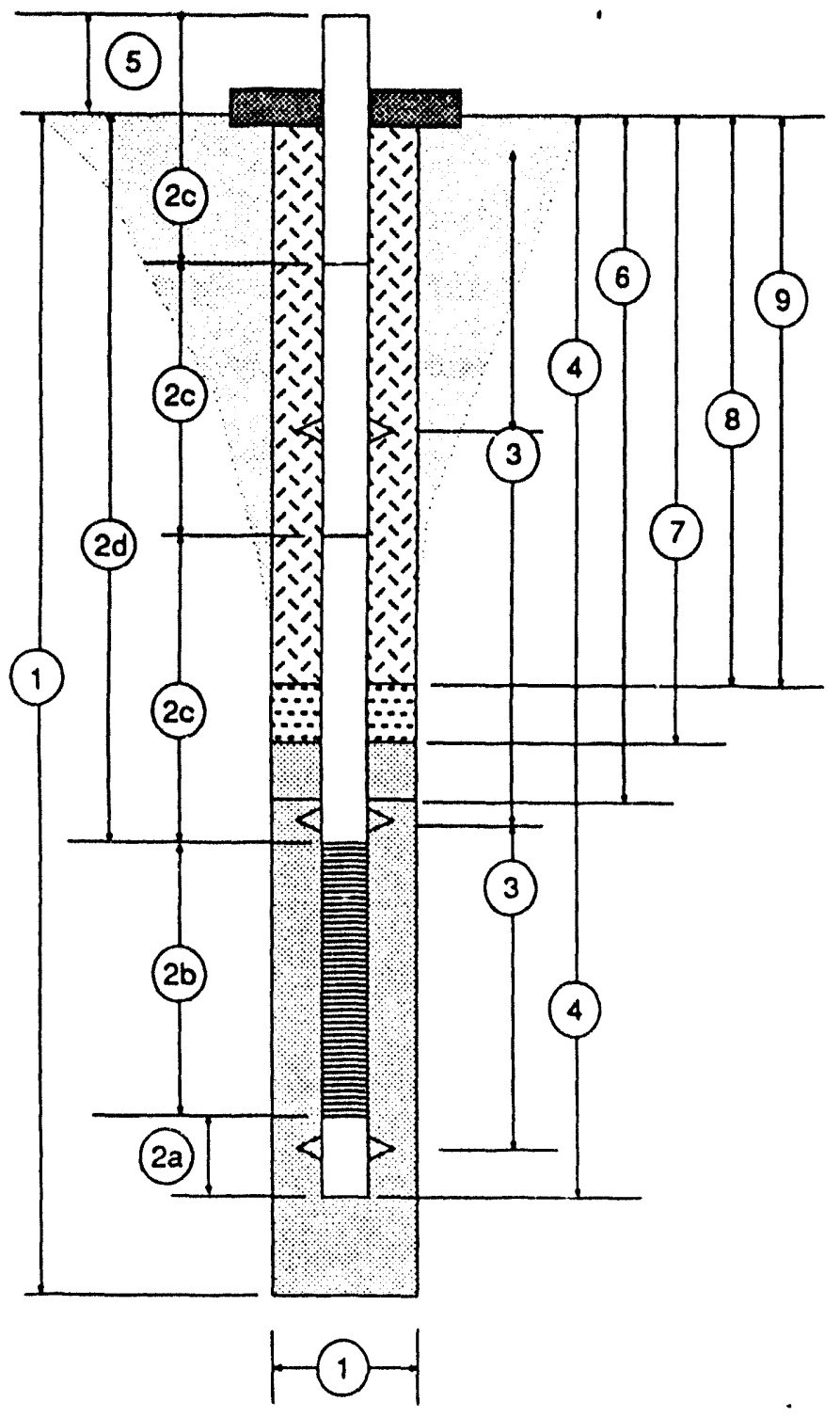

WELL NUMBER

$M H V-20 A$

SRS COORDINATES

SANITARY SEAL ELEVATION

NOTE: ALL MEASUREMENTS

ARE FROM GROUND

SURFACE AT START

OF BORING - MEASUREMENTS

TO NEAREST 0.1 FOOT.

1) Total Drilled Depth/Hole Diameter $60.0^{\circ} / 814^{\circ}$.

2) Casing/Screen Tally (Measured to Nearest 0.01 Fool)

(a) Plug Length $0.02^{\circ}$

(b) Screen Length

$4.90^{\circ}$

(c) Casing Joint Lengths (Measured in Up-

hole Sequence From Top of Screen) 1) $9.88^{\circ}$

2) $9.89^{\circ}$ 3) $9.88^{\circ}$ 4) $9.9^{\circ}$

5) $8.44^{\circ}$

(d) Depth to Top of Screen

$48.00^{\circ}$

3) Depths to Centralizers Not reguired.

4) Total Depth of Installed Well

$52.9^{\circ}$

5) Casing Stick Up (Standard 2.5 A.G.S.) _ /.

6) Depth to Top of Filter Pack

47.6

7) Depth to Top of Fine Sand Səal

Not reguired

8) Depth to Top of Bentonite Seal Not reguired

9) Thickness of Grout 2.4 


\section{MONITORING WELL CONSTRUCTION DIAGRAM}

DRILLING SUBCONTRACTOR EMTC

DRILLER

Blake Cabit

DATE OF WELL INSTALLATION

$11 / 20 / 92$

TECH. O.S.ICO.NAME B.ll Joyce/SEC Donohue

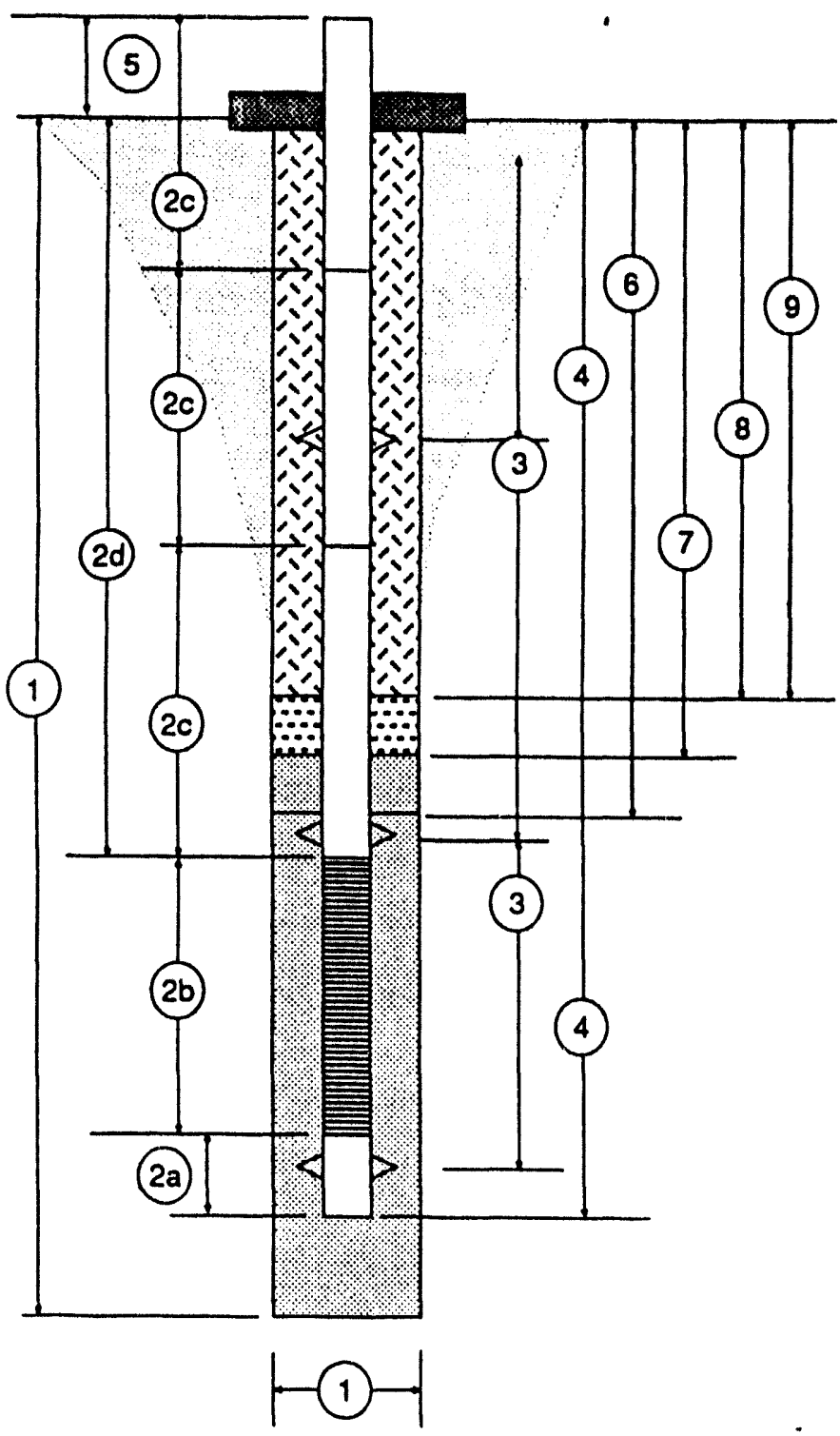

WELL NUMBER_MHV-20B

SRS COORDINATES

SANITARY SEAL ELEVATION

NOTE: ALL MEASUREMENTS

ARE FROM GROUND

SURFACE AT START

OF BORING - MEASUREMENTS

TO NEAREST 0.1 FOOT.

First Grout

1) Total Drilled Depth/Holo Diameter $4 r_{2} / 8 \% "$

2) Casing/Screen Tally (Measured to Nearest 0.01 Foot)

(a) Plug Length $\quad 0.02^{\circ}$

(b) Screen Length $4.89^{\circ}$ (c) Casing Joint Lengths (Measured in Up- 1$) 9.88^{\prime}$
hole Sequence From Top of Screen) 2) $9.88^{\circ}$ 3/ $9.89^{\circ}$ 4/ 8.35

(d) Depth to Top of Screen $38.00^{\circ}$

3) Depths to Centralizers Not reguired

4) Total Depth of Installed Well 42.9

5) Casing Stick Up (Standard 2.5' A.G.S.)

6) Depth to Top of Filter Pack o/ 3.6. $36.3^{\prime}$

7) Depth to Top of Fine Sand Seal _. Not reguired

8) Depth to Top of Bentonite Seal Net reguired

9) Thickness of Grout

$2.7^{\circ}$ 
OAR 30.5

IRe 12.69)

MONITORING WELL CONSTRUCTION DIAGRAM

DRILLING SUBCONTRACTOR EMT

DRILLER

Blake Cabot

DATE OF WELL INSTALLATION

$12 / 02 / 92$

tech. O.S./CO. name $B, 1 /$ Joyce $/ S=C$ Dunutive
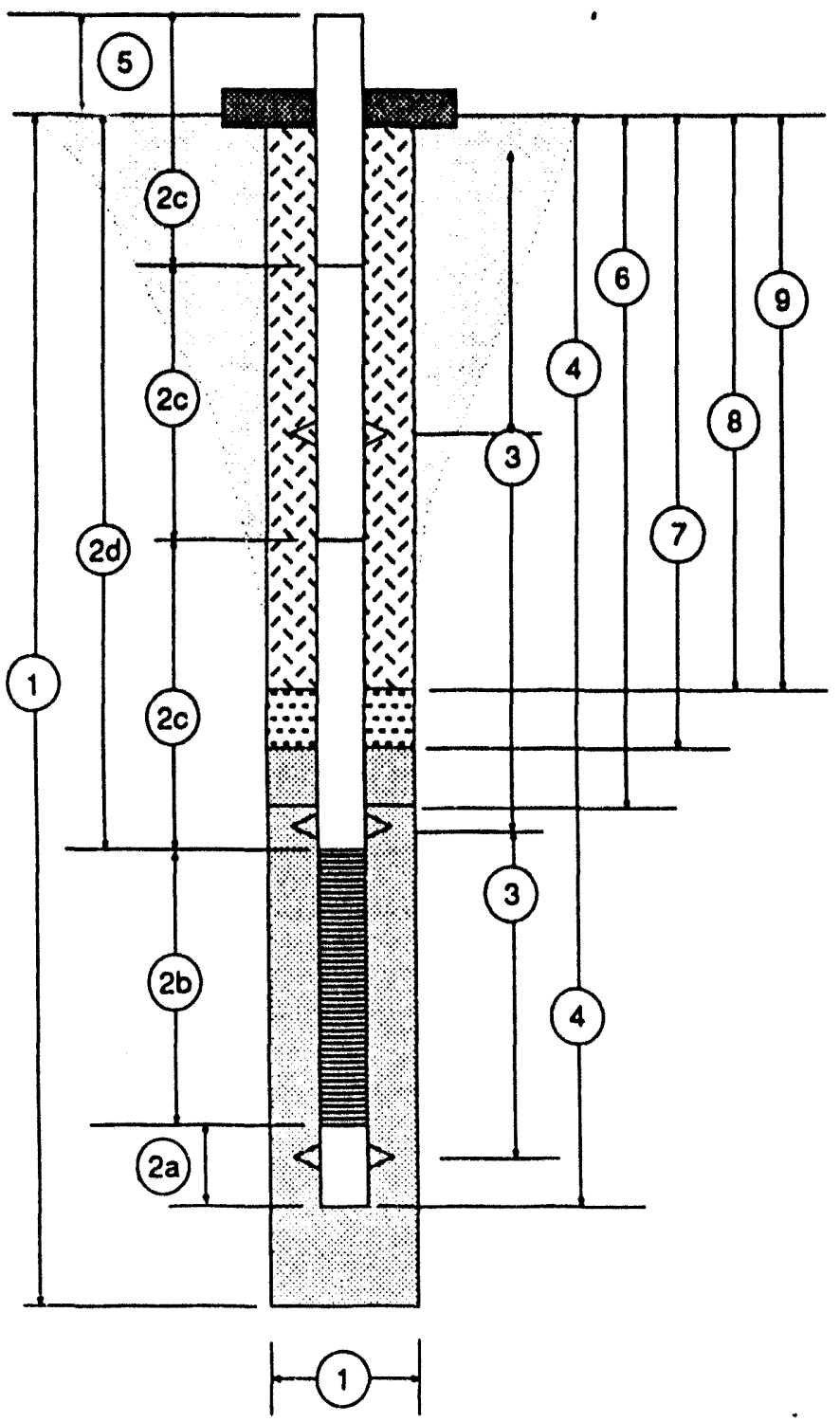

WELL NUMBER $M H L-20 C$

SRI COORDINATES

SANITARY SEAL ELEVATION

NOTE: ALL MEASUREMENTS

ARE FROM GROUND

SURFACE AT START OF BORING - MEASUREMENTS TO NEAREST 0.1 FOOT.

1) Total Drilled Depth/Hole Diameter Secyage Gent

2) Casing/Screen Tally (Measured to Nearest 0.01 Foot)

(a) Plug Length $0.02^{\circ}$

(b) Screen Length $4.90^{\circ}$

(c) Casing Joint Lengths (Measured in Uphole Sequence From Top of Screen) I) $9.92^{\prime}$ 2) $9.92^{\circ} 3 / 8.16^{\prime}$

(d) Depth to Top of Screen $28.00^{\circ}$

3) Depths to Centralizers Not required

4) Total Depth of Installed Well $32.9^{\circ}$

5) Casing Stick Up (Standard 2.5' A.G.S.) $1.8^{\circ}$

6) Depth to Top of Filter Pack 26.3

7) Depth to Top of Fine Sand Seal Not reguiced

8) Depth to Top of Bentonite Seal Not required

9) Thickness of Grout 26.3 


$$
\text { PAGE } 2 \text { O } 2
$$

SEC DONOHUE.

CALCULATION SHEET

CLIENT WSRC LOCATION SR S JOB NO. G4346 subject RE Heating Test - MHV - 20 Piezometers Casing Tally BY Bill Joyce DATE $12 / 02 / 92$ CHECKED BY DATE

$\frac{\text { Casing }}{\text { Tally }} \frac{A}{9.88^{\circ}} \cdot \frac{\text { Screen }}{\frac{A}{48.0^{\circ}-53.0^{\circ}}}$

2) $9.89^{\circ}$

3) $9.88^{\circ}$

4) $9.91^{\circ}$

5) $\frac{9.88^{\circ}}{49.44^{\circ}}$

$\frac{-48.00^{\circ}}{1.44^{\circ}}$ Top of Casing

$1.44^{\circ}$ Stich -up

1) $\frac{B}{9.88^{\circ}}$

4) $9.88^{\circ}$

3) $9.89^{\circ}$

4) $\frac{9.91^{\circ}}{39.56^{\circ}}$

$-38.00^{\circ}$. Top of Cawing $1.56^{\circ}$ Sticker

1) $\frac{C}{9.92^{\circ}} \quad \frac{C}{28.0^{\circ}-33.0^{\circ}}$

2) $9.92^{\circ}$

3) $\frac{9.92^{\circ}}{29.10^{\circ}}$

$-2800^{\circ}$ Top of Casing
Materials

List $(A, B C)$

$\frac{\text { Casing }}{1 " \text { Pvc }}$

Schedule yo

Tri-Lob Threads

Flush

Tinco Brand

$\frac{\text { Screen }}{1 \text { PVC }}$

slotted

0.010 in. slot

Flush

Tri-Loc Threads

Times Brand

Sump

Not required

plug

I"puc

Schedule yo

Tri-Lue Treads

Flush

Timco Brand
Screen / plug

Tally

A

\begin{tabular}{l|l|l|}
\hline \multirow{4}{*}{$4.90^{\circ}$} & $\equiv$ & $0.09^{\circ}$ \\
& $\equiv$ & $4.68^{\circ}$ \\
& $\equiv$ & \\
\cline { 2 - 3 } & & $0.13^{\circ}$ \\
\hline $0.02^{\circ}$ & Plug & $0.02^{\circ}$ \\
\hline
\end{tabular}

$B$

\begin{tabular}{l|l|l|}
\hline \multirow{4}{*}{$4.89^{\circ}$} & & $0.08^{\circ}$ \\
& $=4.68^{\circ}$ \\
& $=$ & \\
\cline { 2 - 3 } & & $0.13^{\circ}$ \\
\hline $0.02^{\circ}$ plug & $0.02^{\circ}$ \\
\hline
\end{tabular}

$c$

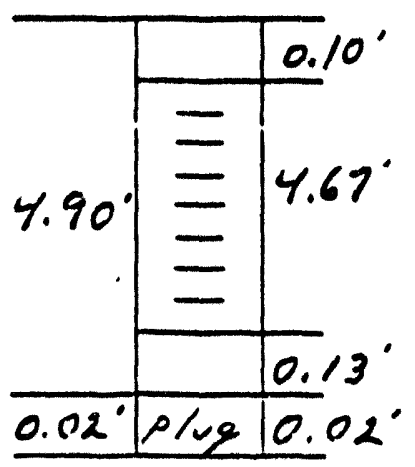


PAge 1 OF 2

SEC DONOHUE

CLIENT WSRC SUBJECT RF Heating Test - MHV - 20 BY Bill Joyce DATE $12 / 02 / 12$ CHECKED BY CALCULATION SHEET LOCATION SRS JOB NO. G.4346 Piezometer Well Construction Diagram DATE

from. $\quad M H V-20$

Dotton

Casing frum

Grovad

Surtuce
Gravel Pack

Based on Practical

Application in the Field of ibag Coarse Sand $21.2 \mathrm{ft}^{3}$ bag Grobt $=0.6 \mathrm{ft}^{3}$

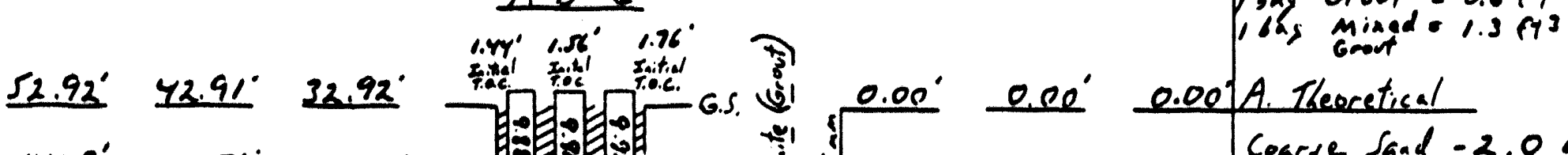

1.4y' $1.56^{\circ} 1.76$

$44.48^{\circ} 34.56^{\circ} 24.26^{\circ}$

$34.57^{\circ} \quad 24.67^{\circ} \quad 14.84^{\circ}$

$24.69^{\circ} \quad 14.79^{\circ} \quad 4.92^{\circ}$

$\frac{14.80^{\circ}}{\frac{4.92^{\circ}}{0.02^{\circ}}} \frac{4.91^{\circ}}{\frac{0.02^{\circ}}{\left(0.00^{\circ}\right)}} \frac{\frac{0.02^{\circ}}{\left(0.00^{\circ}\right)}}{(0.0)^{2}}$

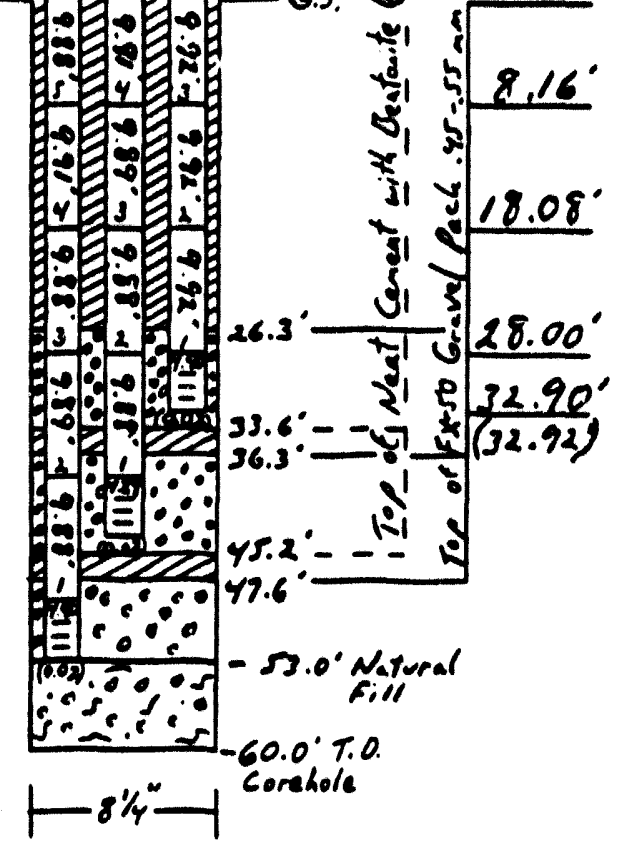

$+17$
Coarse Sand - 2.06 Grout - 0.6 bags Actual

Coarse Sand - 2.06 Grout - 1 bag

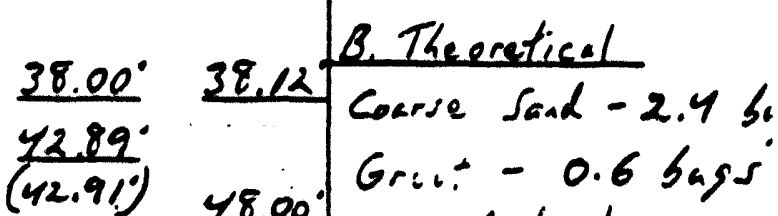
$18.00^{\circ}$ Actual

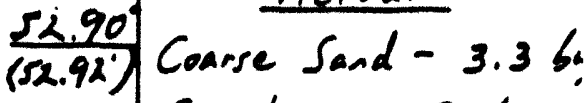
Grout - 0.7 bagd C Theocetical Coarse Sand- 2.36 b Grout -9.2 bass ay Actual

Cuarse Sand- $2.3 \mathrm{bag}$ Grout - 6.7 bags 
DRILLING SUBCONTRACTOR EMTC DRILLER Steve Roesi DATE OF WELL INSTALLATION $1 / 21 / 93$ TECH. O.S.ICO.NAME Bill Tuyce/SEC Dindure

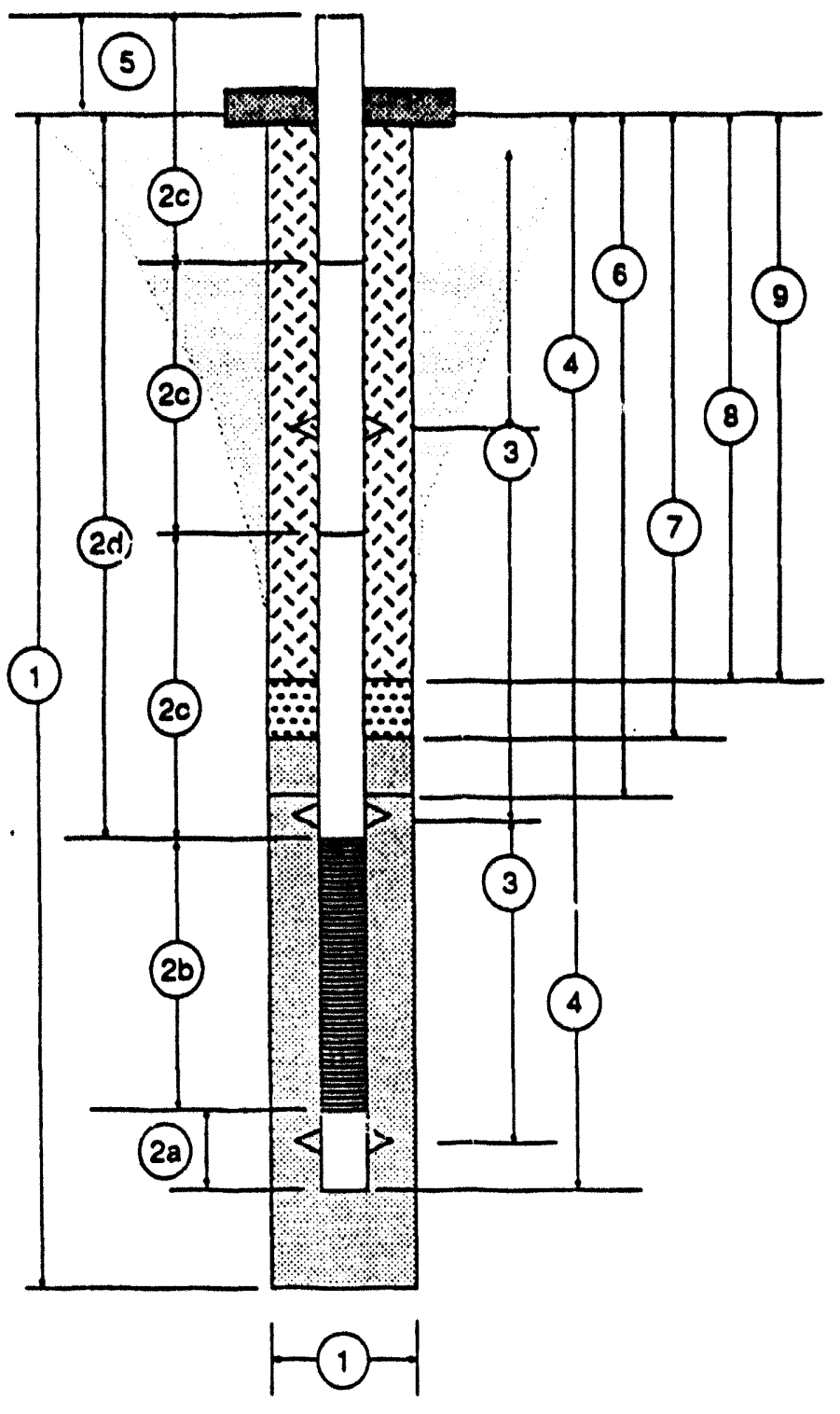

WELL NUMBER_MHV - $20 T$ SRS COORDINATES

SANITARY SEAL ELEVATION

NOTE: ALL MEASUREMENTS

ARE FROM GROUND SURFACE AT START OF BORING - MEASUREMENTS TO NEAREST 0.1 FOOT.

1) Total Drilled Depth/Hole Diamoter $\frac{63.0^{\circ} / 8^{\circ}}{-}$ 2) Casing/Screen Tally (Measured to Nearest 0.01 Foot)

(a) Sump a Plug Longth Nut ceguiced

(b) Screen Length Net reguired

(c) Casing Joint Lengths (Measured in Uphole Sequence From Top of Screen) 1$) 10.12^{\circ}$ includiase threads 2/ 10.00 3/10.00 की $10.00^{\circ}$ 5) $9.99^{\circ}$ c) $4.89^{\circ}$

(d) Depth to Sepotsersen Fitere eatics: $28.00^{\circ} 33.00^{\circ}$ $\pi 7.00^{\circ} .43 .00^{\circ}, 48.00^{\circ}$ and $53.00^{\circ}$. Rermecoyeles : $28.00^{\circ}$. $38.00^{\circ} \mathrm{and} 48.00^{\circ}$.

3) Dopths to Contralizers_N_Not reguiced

4) Total Depth of Installed Well $25.0^{\prime}$

5) Casing Stick Up (Standard 2.5' A.G.S.) $5.1^{\circ}$

6) Depth to Top of Filter Pack $10.2^{\circ}$

7) Depth 10 Top of Fine Sand Seal Not reguired 9! Nonth in Tng nt Roninnito Saal._._. Nut required

9) Thickness of Grout $10.2^{\prime}$ 
FIELD GEOLOGIC LOG

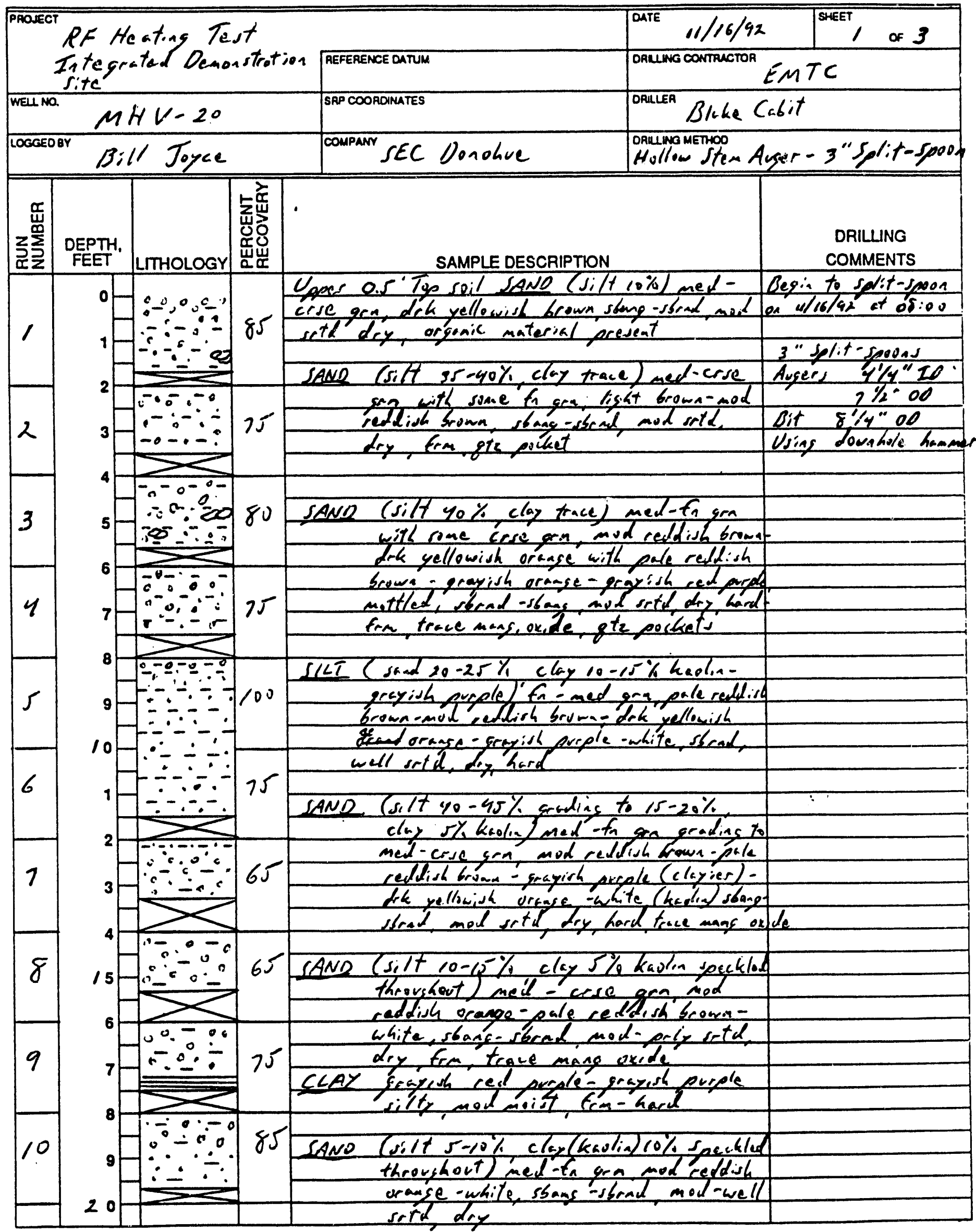


FIELD GEOLOGIC LOG

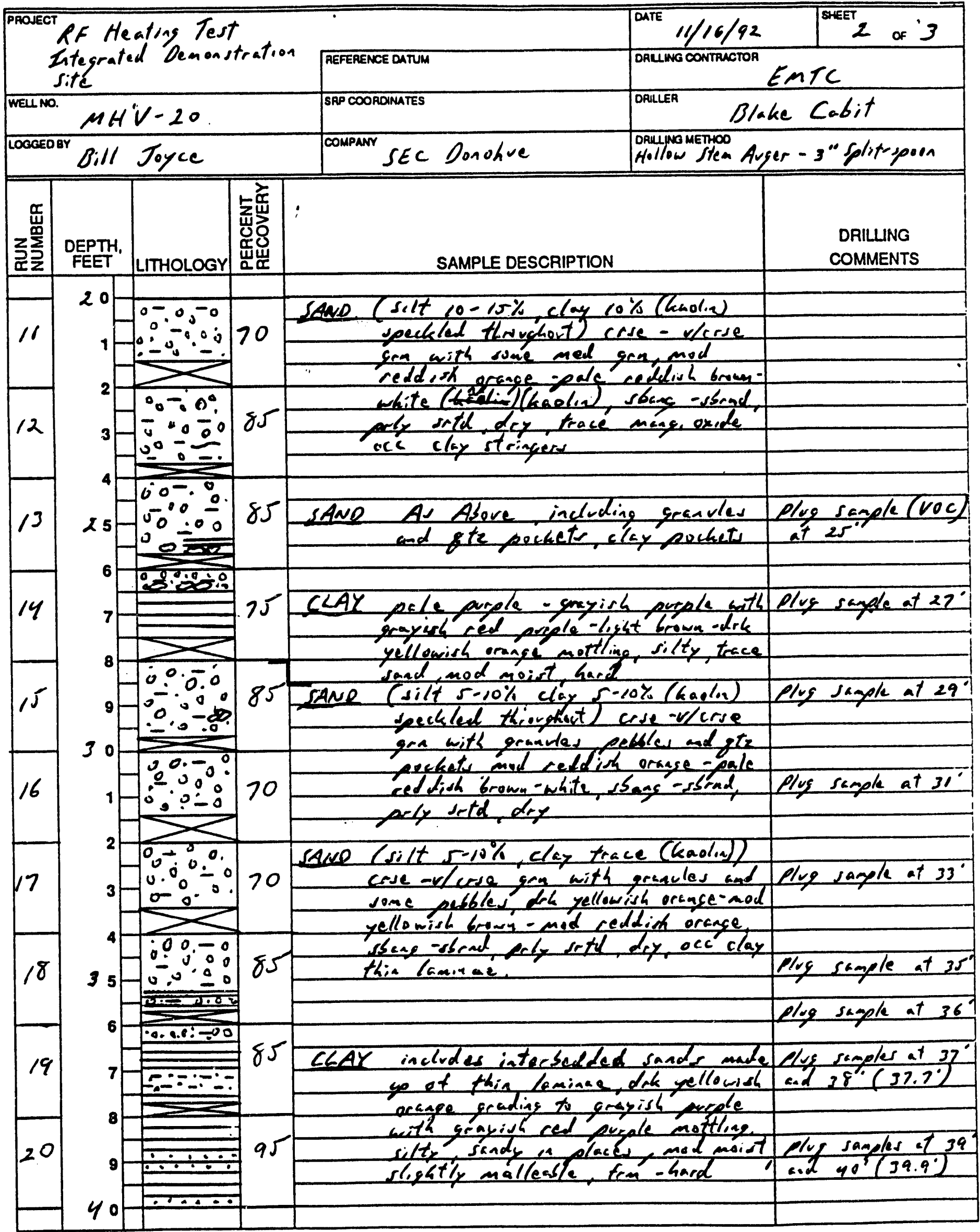


FIELD GEOLOGIC LOG

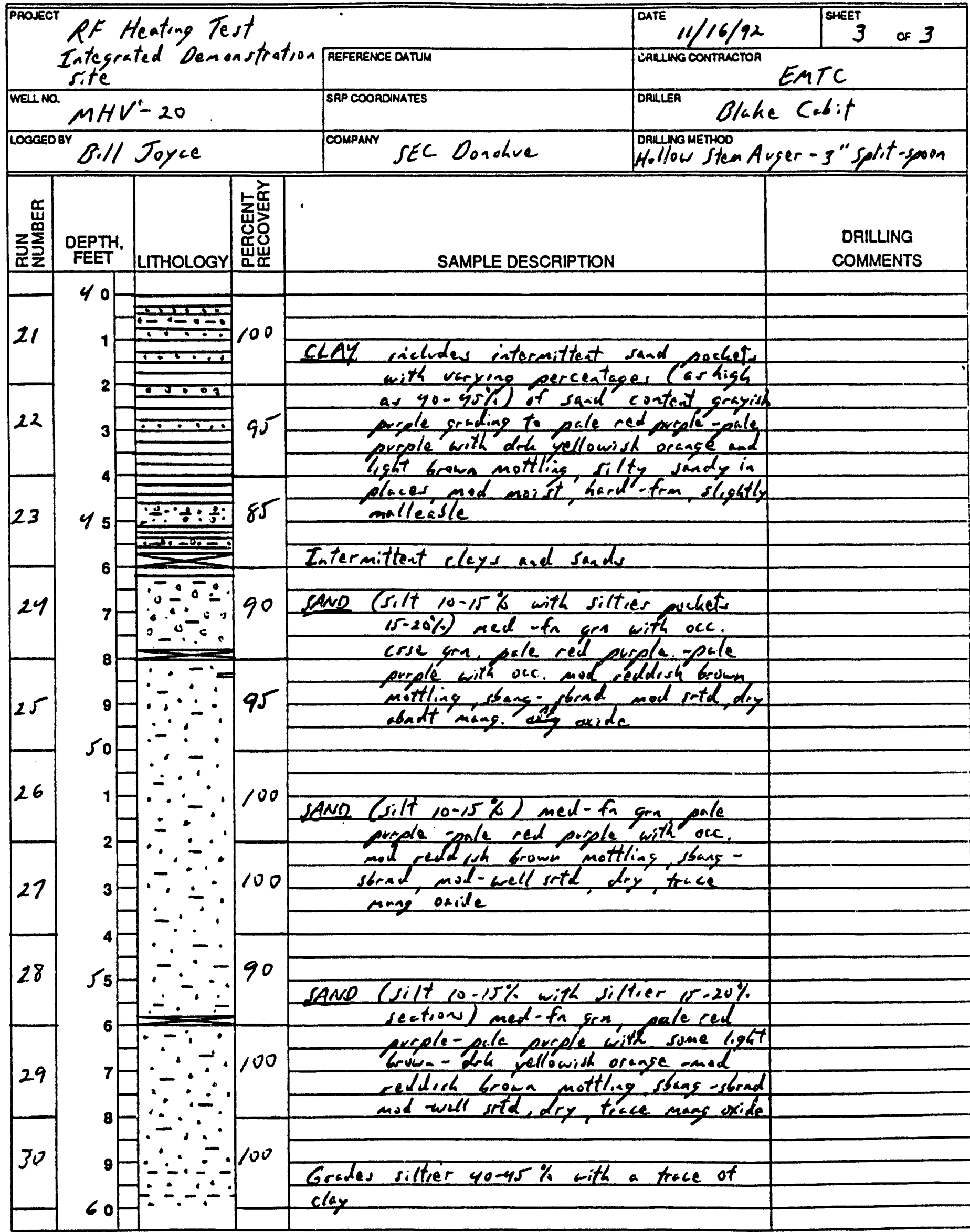


DRILLING SUBCONTRACTOR LA9TC DRILLER Terry ficasón

DATE OF WELL INSTALLATION $11 / 13 / 92$ TECH. O.S./CO. name B.11 Jujce/SEC Donohve

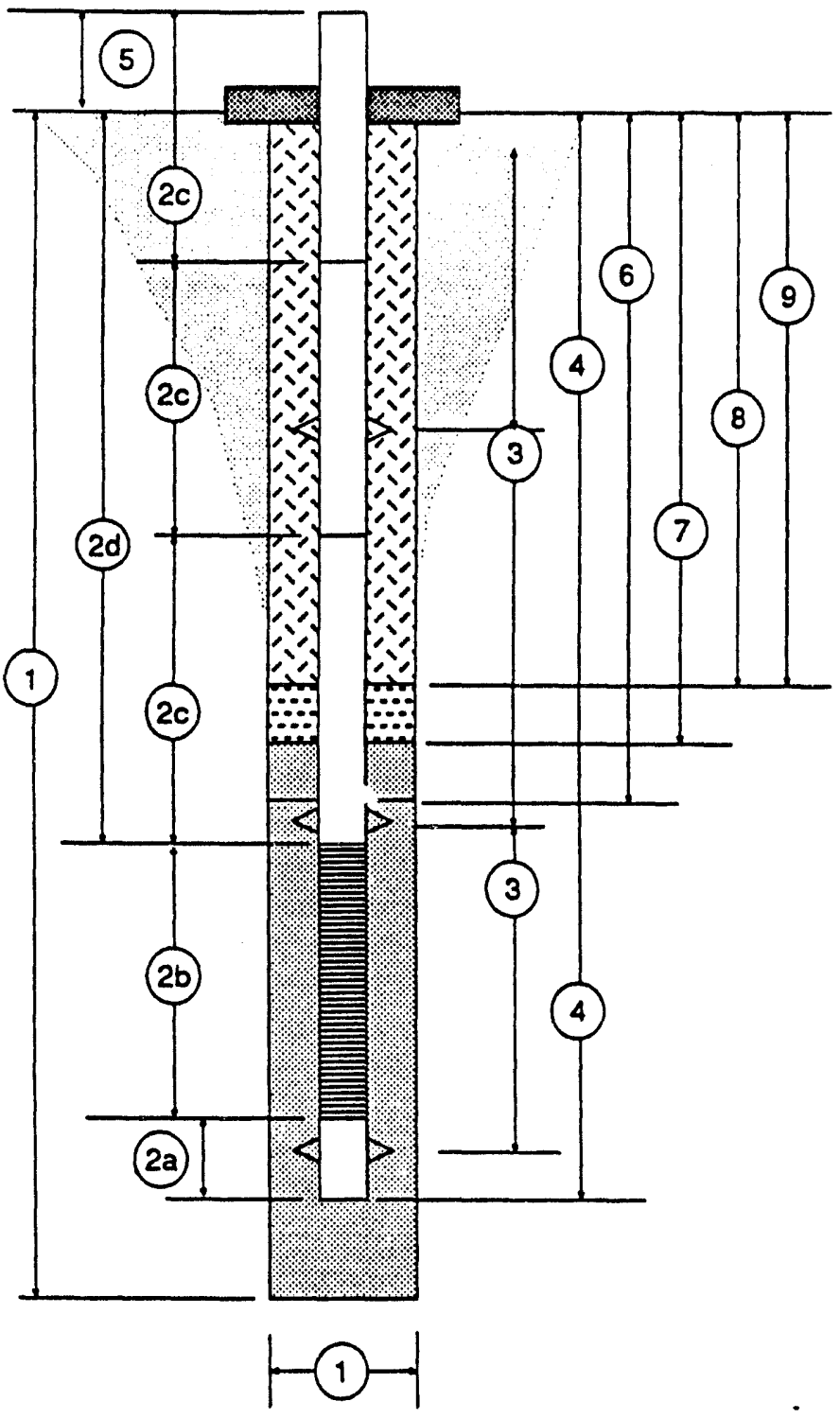

WELL NUMBER $M N^{\prime} V \cdot 2 / \angle$ SRS COORDINATES

SANITARY SEAL ELEVATION

NOTE: ALL MEASUREMENTS

ARE FROM GROUND

OF BORING - MEASUREMENTS

TO NEAREST 0.1 FOOT.

(d) Depth to Top of Screen__ 48,00

3) Depths to Centralizers _ Not re suired

4) Total Depth of Installed Well

52.9

5) Casing Stick Up (Standard 2.5' A.G.S.)

6) Depth to Top of Filter Pack 46.3

7) Depth to Top of Fine Sand Seal Not reguired 9) nonth !n Ton at Roninnito Soal Not.. reguired

9) Thickness of Grout $2 \cdot 5^{\prime}$ 
DRILLING SUBCONTRACTOR EMTC DRILLER Brake Cabit

DATE OF WELL INSTALLATION $11 / 16 / 12$ TECH. O.S.ICO. NAME Bill Toyce / SEC Donchue

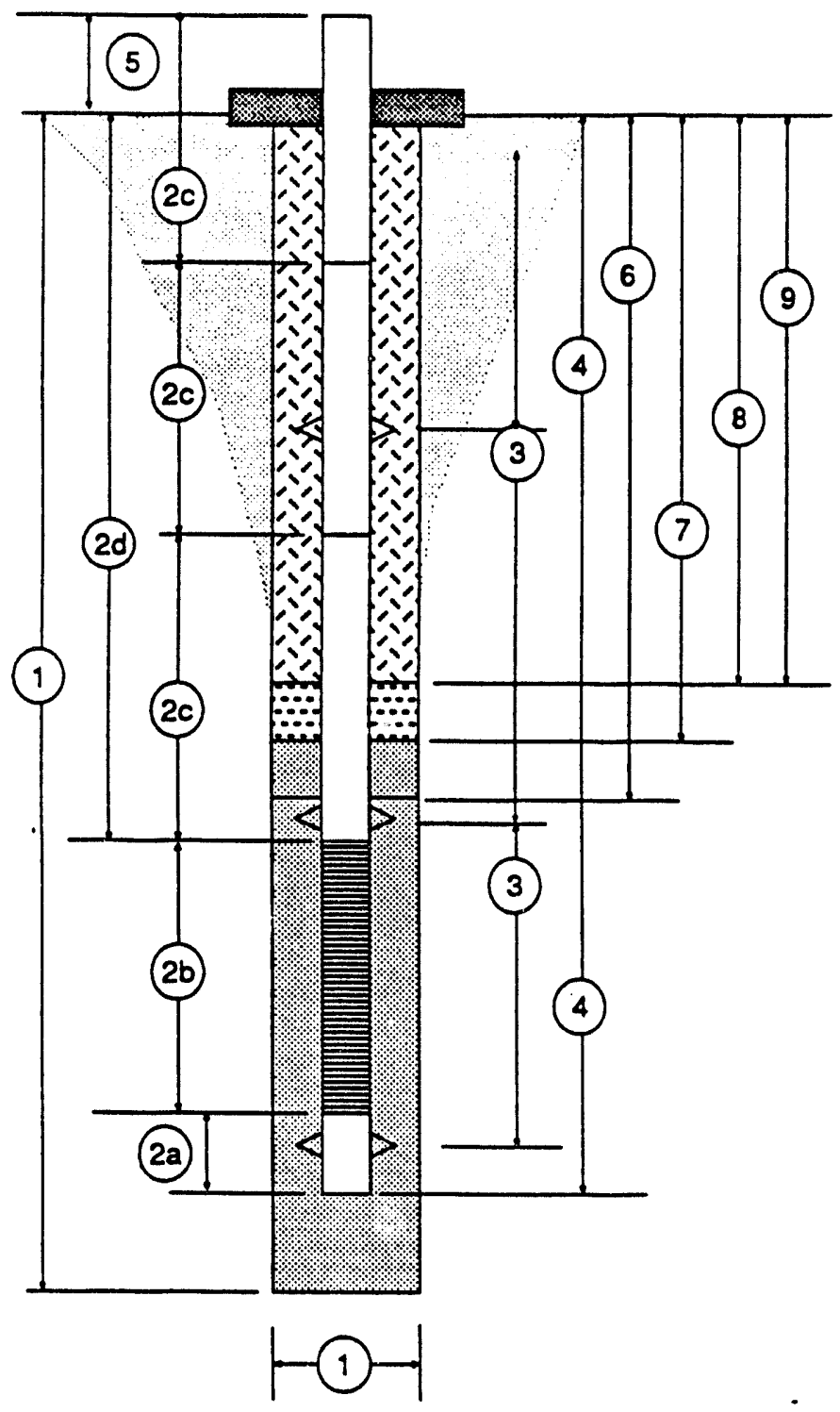

WELL NUMBER MHV-2,B SRS COORDINATES

SANITARY SEAL ELEVATION

NOTE: ALL MEASUREMENTS ARE FROM GROUND SURFACE AT START OF BORING - MEASUREMENTS TO NEAREST 0.1 FOOT.

$$
\text { fird Ge..t }
$$

$$
\text { stage }
$$

1) Total Drilled DepthHole Diameter $43,8^{\circ} / 81 / 4 "$

2) Casing/Screen Tally (Measured to Nearest 0.01 Foot)

(a) Piug Length

$0.02^{\circ}$

(b) Screen Length $1.90^{\circ}$

(c) Casing Joint Lengths (Measured in Uphole Sequence From Top of Screen) $29.90^{\circ}$ 2) $9.92^{\circ}$ 3) $9.88^{\circ}$ 4) $8.30^{\circ}$

(d) Depth to Top of Screen $38.00^{\circ}$

3) Depths to Centralizers Not reguired

4) Total Depth of Installed Well $42.9^{\circ}$

5) Casing Stick Up ('otandard 2.5' A.G.S.) 1.6

6) Depth to Top of Finer Pack 36.7

7) Depth to Top of Fine Sand Seal Not reguired

9) Depth to Ton c! Donicnits Seal Not reguired.

9) Thickness of Grout 


\section{MONITORING WELL CONSTRUCTION DIAGRAM}

DRILLING SUBCONTRACTOR EMTC

DRILLER

Blake Cabit

DATE OF WELL INSTALLATION $12 / 03 / 92$

TECH. O.s.lco. name Bill Joyce/sEC Doruhve

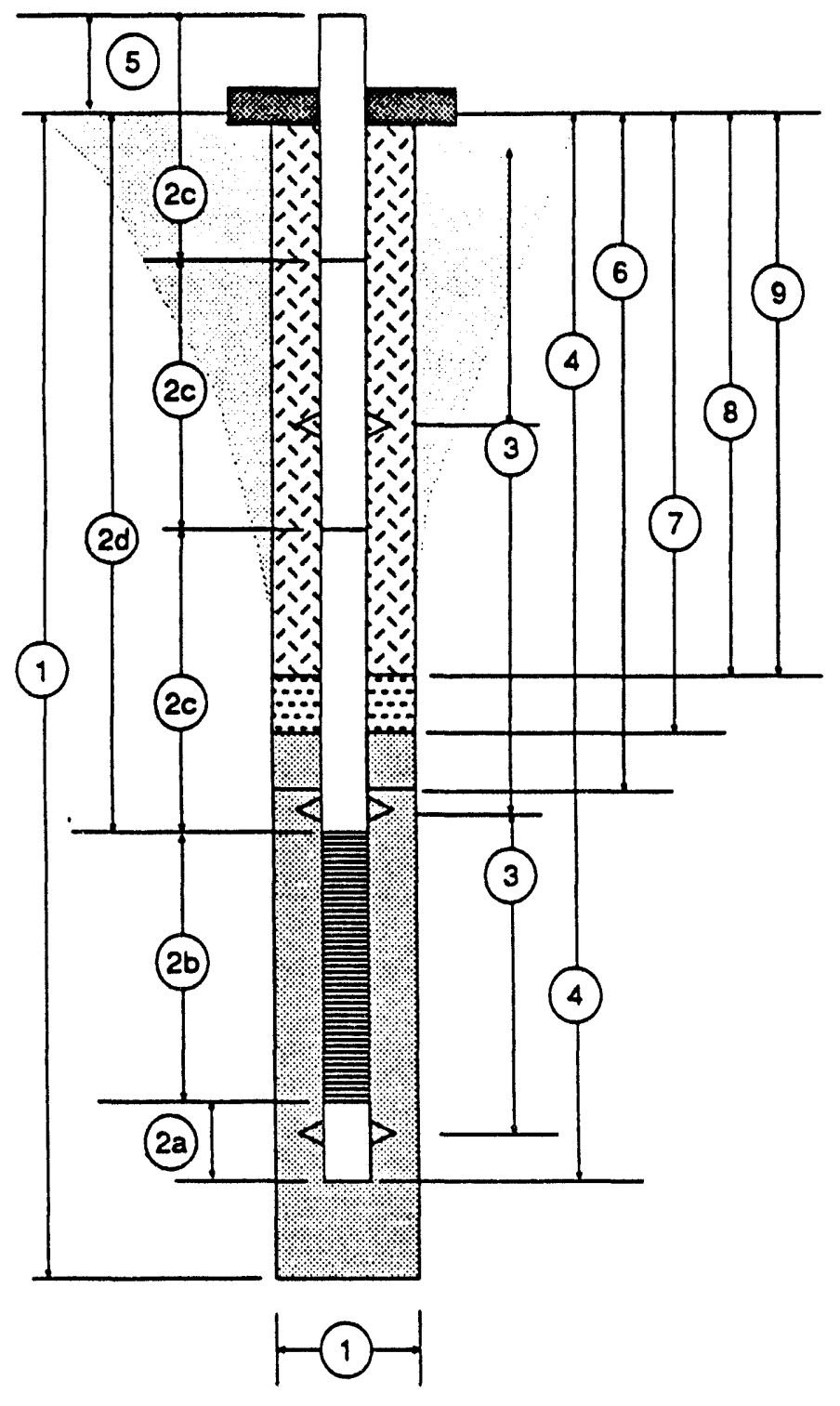

WELL NUMBER MHV-2/C

SRS COORDINATES

SANITARY SEAL ELEVATION

NOTE: ALL MEASUREMENTS

ARE FROM GROUND

SURFACE AT START

OF BORING - MEASUREMENTS

TO NEAREST 0.1 FOOT.

1) Total Drilled Depth/Hole Diameter $\begin{gathered}\text { Sccoad Gro.t } \\ \text { T4aye } \\ 34.3\end{gathered} / 8 \% "$

2) Casing/Screen Tally (Measured to Nearest 0.01 Foot)

(a) Plug Length

$0.04^{\prime}$

(b) Screen Length

4.90 (c) Casing Joint Lengths (Measured in Up-
hole Sequence From Top of Screen)
2) $9.90^{\circ}$
3) $8.19^{\circ}$

(d) Depth to Top of Screen

$28.00^{\circ}$

3) Depths to Centralizers Not reguired

4) Total Depth of Installed Well $32.9^{\circ}$

5) Casing Stick Up (Standard 2.5' A.G.S.) $1.7^{\circ}$

6) Depth to Top of Fitter Pack $26.5^{\circ}$

7) Depth to Top of Fine Sand Seal Not reguired

8) Dะzin :

Int reg.t.red

9) Thickness of Grout

$26.5^{\circ}$ 
PAGE

of 2

SEC DONOHUE

CLIENT WSRC
CALCULATION SHEET

LOCATION SR JOB NO. G. 4346 BY Bill Joyce DATE $12 / \mathrm{cij}^{-1} / \mathrm{i}^{2}$ CHECKED BY DATE

From.

Bottom

Casing

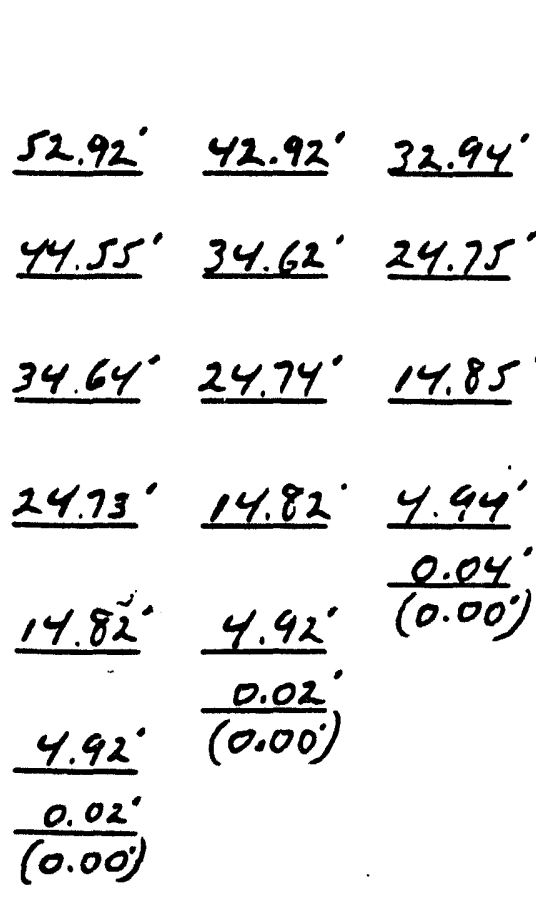

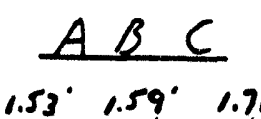

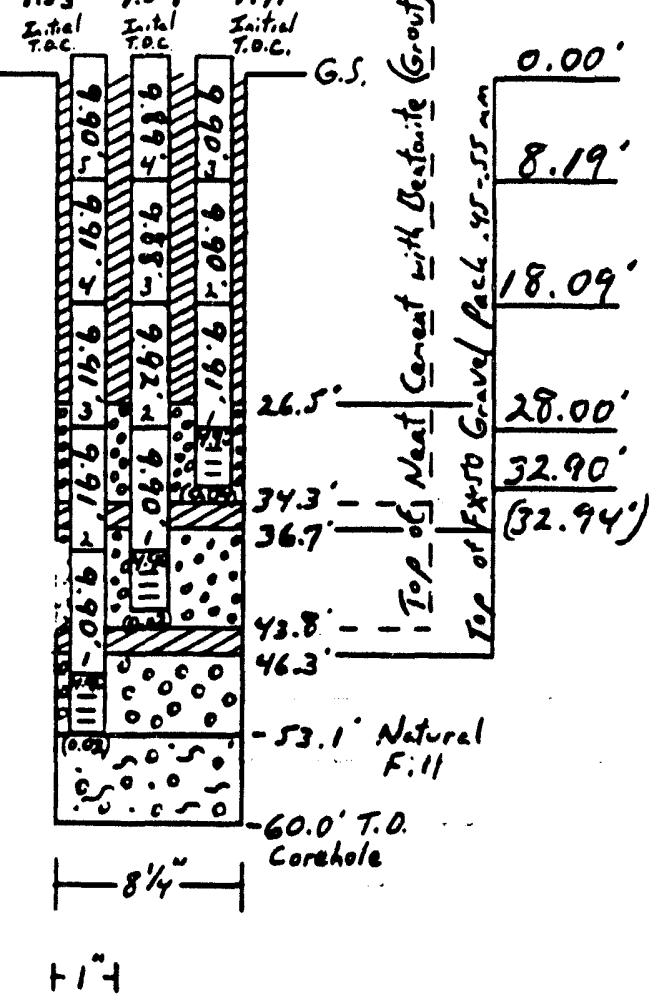

from

Grovel

Ser face
Grave/ Pack

Based on Practical

Application in the Field of

1. bag Coarse Sand l=1.2 $\mathrm{ft}^{3}$

bang Gorge $=0.6 \mathrm{ft}^{3}$

b as Mined : $1.3 \mathrm{ft}^{\circ}$

$0.00^{\circ} 0.00^{\circ}$ A. Theoretical

Coarse Sand -2.0 bes

Grout - 0.6 bags

$\frac{\text { Actual }}{\text { Sand }-2.5 \mathrm{bas}}$

Coarse Sand -2.5 bes
Grout -0.7 bags

$28.10^{\circ} \quad 28.19$

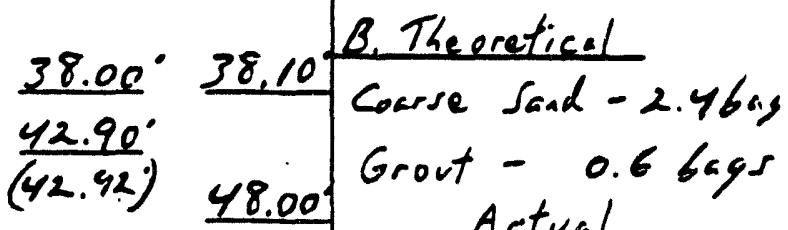

$\frac{\text { Actual }}{\text { Sand - 2.5 haj }}$

Grout - 0.7 bags

C Theoretical

Coarse Sand -2.3 bs,

Grout - 7.2 bags

Actual

Coarse Sand - 2 bags

Grout - 6.7 bugs 


$$
\text { PAGE } 2 \text { OF } 2
$$

SEC DONAHUE

CLIENT WSRC
CALCULATION SHEET

LOCATION

SeAS

JOB NO. G4346

SUBJECT RE Heating Test - MHV - 21

Pedometers Casing Tally

BY Bill Joyce DATE $12 / 03 / 92$ CHECKED BY DATE

$\frac{\text { Casing }}{\text { Tally }} \frac{A}{\frac{A}{9.90^{\circ}}} \frac{\begin{array}{l}\text { Screen } \\ \text { Zones }\end{array}}{\frac{A}{48.0^{\circ}-53.0}}$

2) $9.91^{\circ}$

3) $9.91^{\circ}$

4) 9.91

5) $\frac{9.90^{\circ}}{49.53^{\circ}}$

$-48.00^{\circ}$ Top of Casing

1.53 stick-up

1) $\frac{B}{9.90^{\circ}}, \frac{B}{38.0^{\circ}-43.0^{\circ}}$

2) $9.92^{\circ}$

3) $9.88^{\circ}$

4) $\frac{9.89^{\circ}}{39.59}$

$-38.00^{\circ}$ Top of Cawing $1.59^{\prime}$ stick -up

Materials

List $(A, B C)$

$\frac{\text { Casing }}{1 " \text { Pvc }}$

Schedule yo

Tri-Loc Threads

Flush

Tinco Brand

$\frac{\text { Screen }}{\text { "PVC }}$

Screen / plug

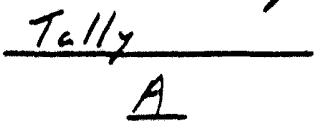

A

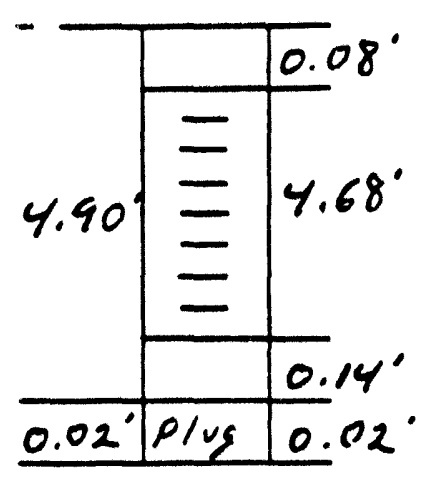

slotted

0.010 in. slot

Flush

Tri-Loc Threads

Times Brand

Sump

Not required

plug

$B$

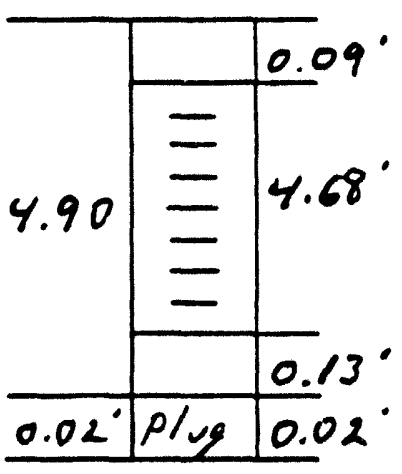

I"puc

Schedule yo

Tri-Loc Threads

Flush

Timco Brand
1) $\frac{c}{9.91^{\circ}} \quad \frac{c}{28.0^{\circ}-33.0^{\circ}}$

2) $9.90^{\circ}$

3) $\frac{9.90^{\circ}}{29.70^{\circ}}$

- 2800 Top of Casing

$1.71^{\circ}$ Stick- yo 
DRILLING SUBCONTRACTOR EMTC

DRILLER

Garry Mosre

DATE OF WELL INSTALLATION

$1 / 15 / 93$
WELL NUMBER M M $-4 V-21 T$

SRS COORDINATES

SANITARY SEAL ELEVATION TECH. O.S.ICO. NAME D.ll Joyce/SEC Donohue

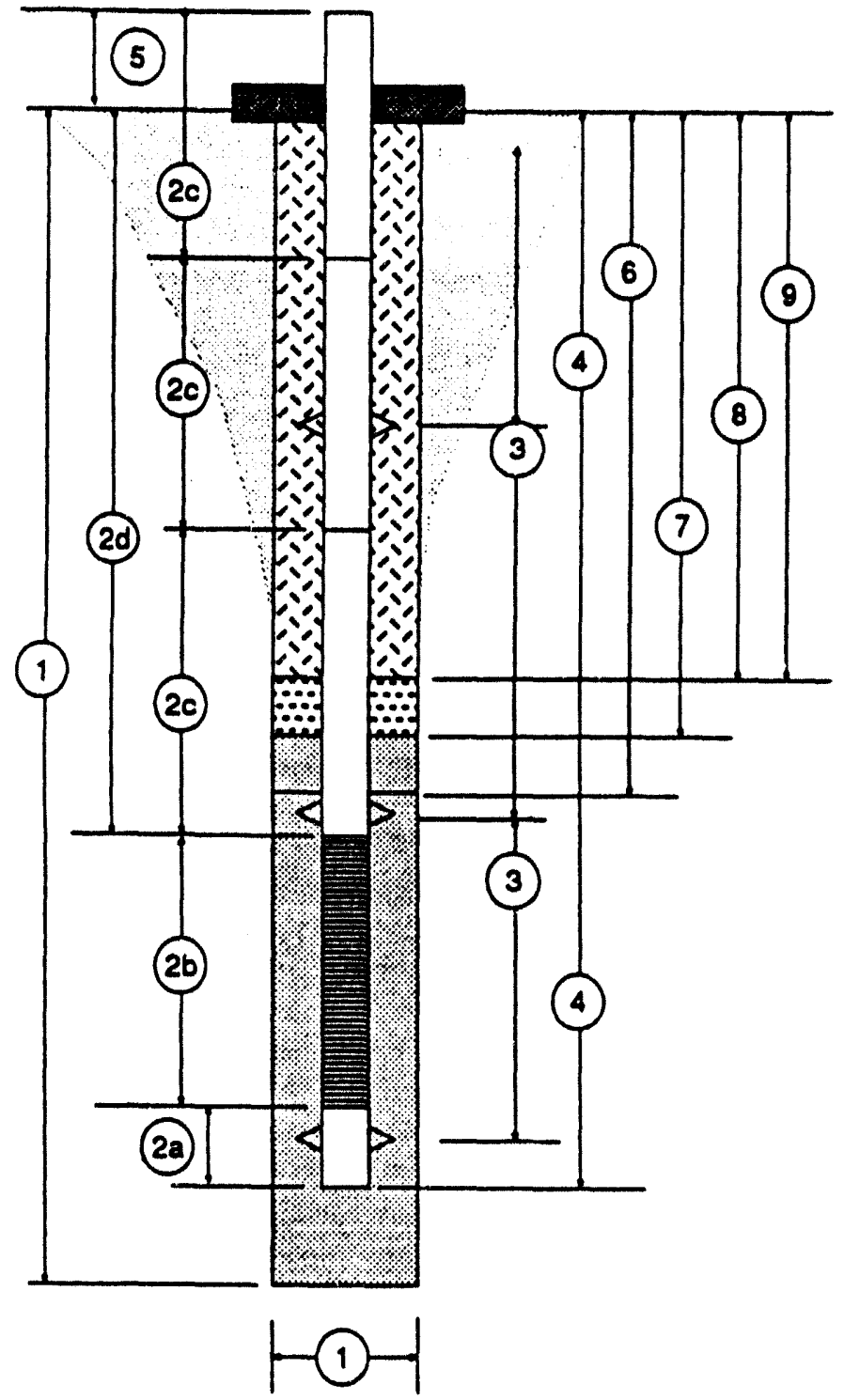

NOTE: ALL MEASUREMENTS

ARE FROM GROUND

SUAFACE AT START

OF BORING - MEASUREMENTS

TO NEAREST 0.1 FOOT.

Fiber Optics Temperature Sansors

1) Total Drilled Depth/Hole Diameter $68.0^{\circ} / 81 / 2^{\circ}$

2) Casing/Screen Tally (Measured to Nearest 0.01 Foot)

(a) Sump \& Plug Length Not regvired

(b) Scroen Longth Net cequired

(c) Casing Joint Longthis (Mossured in Uphole Soquenco From Top of Screen) 1) $10.13^{\circ}$ including theeds $2 / 10.00^{\circ}$ 3/ $10.00^{\circ}$ y) $9.99^{\circ} 5 / 9.99^{\circ}$ c) $9.99^{\circ}$ 7) $1.90^{\circ}$

Seasors Ilermecerdes: 28.00.38.00:0.1 48.00

(d) Dopth :o Topotomen Fiber eptics: $28.00^{\circ} 33.00^{\circ}$ $38.00^{\circ}, 43.00^{\circ}, 48.00^{\circ}$ and $53.00^{\circ}$. Lameence fiveraece: $15.00^{\circ}, 20.00^{\circ} 20.00^{\circ}, 30.00^{\circ}, 33.00^{\circ} 40.00^{\circ}$. 3) Depths to Contralizers NoT regrired

4) Total Depth of installed Woll $62.0^{\circ}$

5) Casing Stick Up (Standard 2.5' A.G.S.)

6) Depth to Top of Filter Pack 9.5

7) Depth 10 Top of Fine Sand Seal Not raguired

8) Depth 10 Top of Bentonine Seal Not recuired

9) Thickness of Grout $\quad 9.5^{\prime}$ 







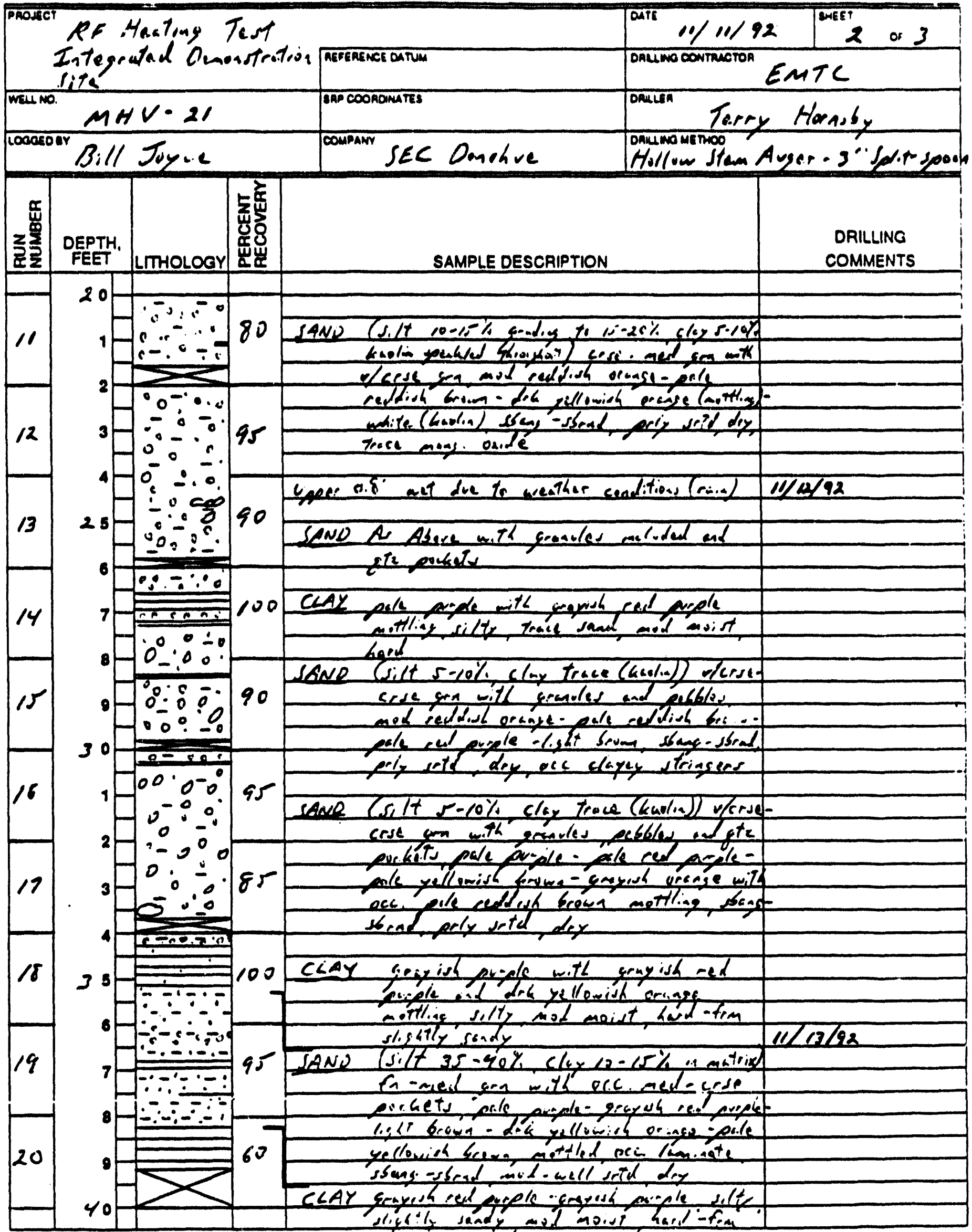


FIELD GEOLOGIC LOG

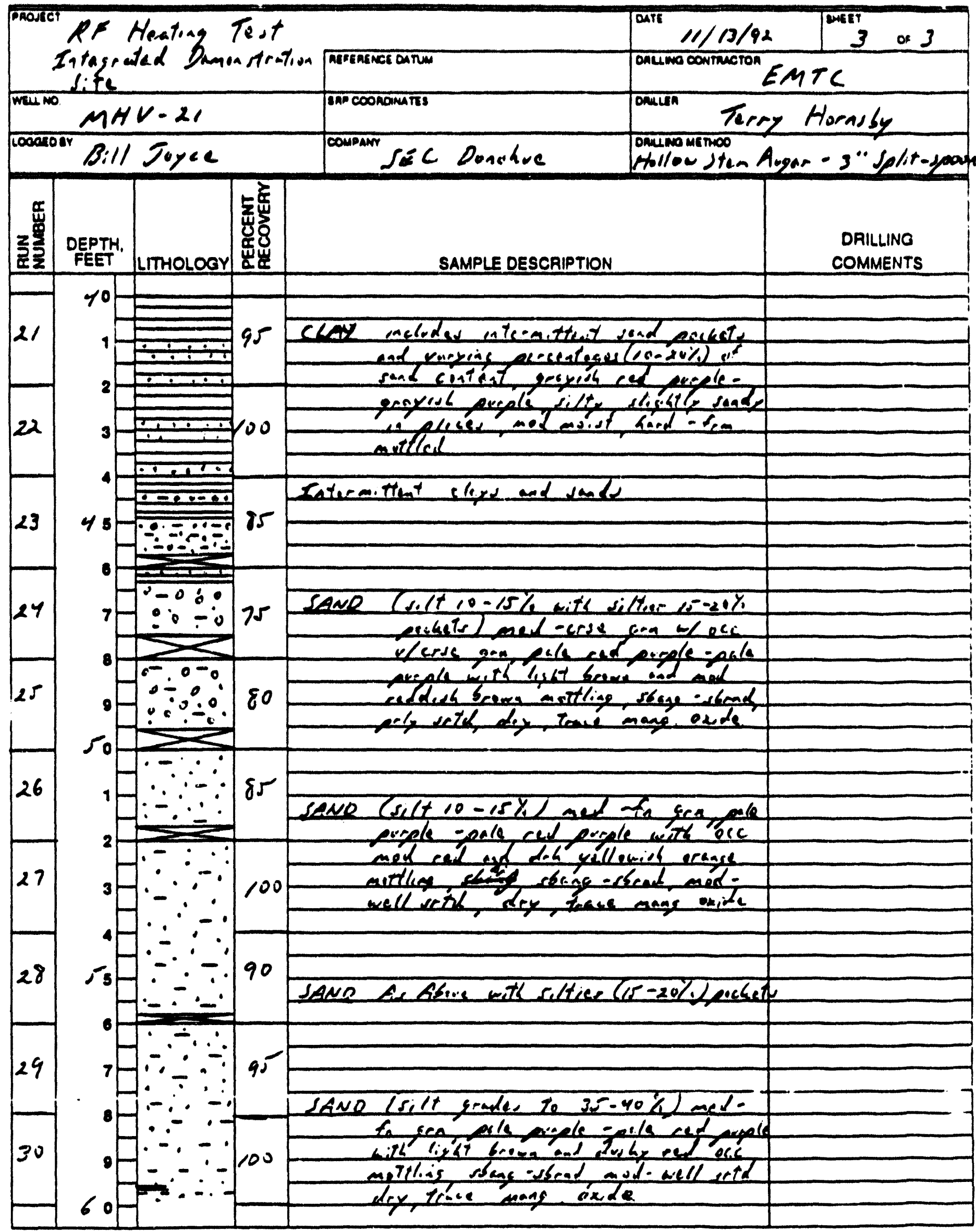


DRILLING SUBCONTRACTOR ENTE

DRILLER

Bjake Cabi,

DATE OF WELL INSTALLATION

$11 / 19 / \% 2$
WELL NUMBER MrV- $M: A$

SRS COORDINATES TECH. O.S.ICO.NAME Rell Jugece/sec Runoúve

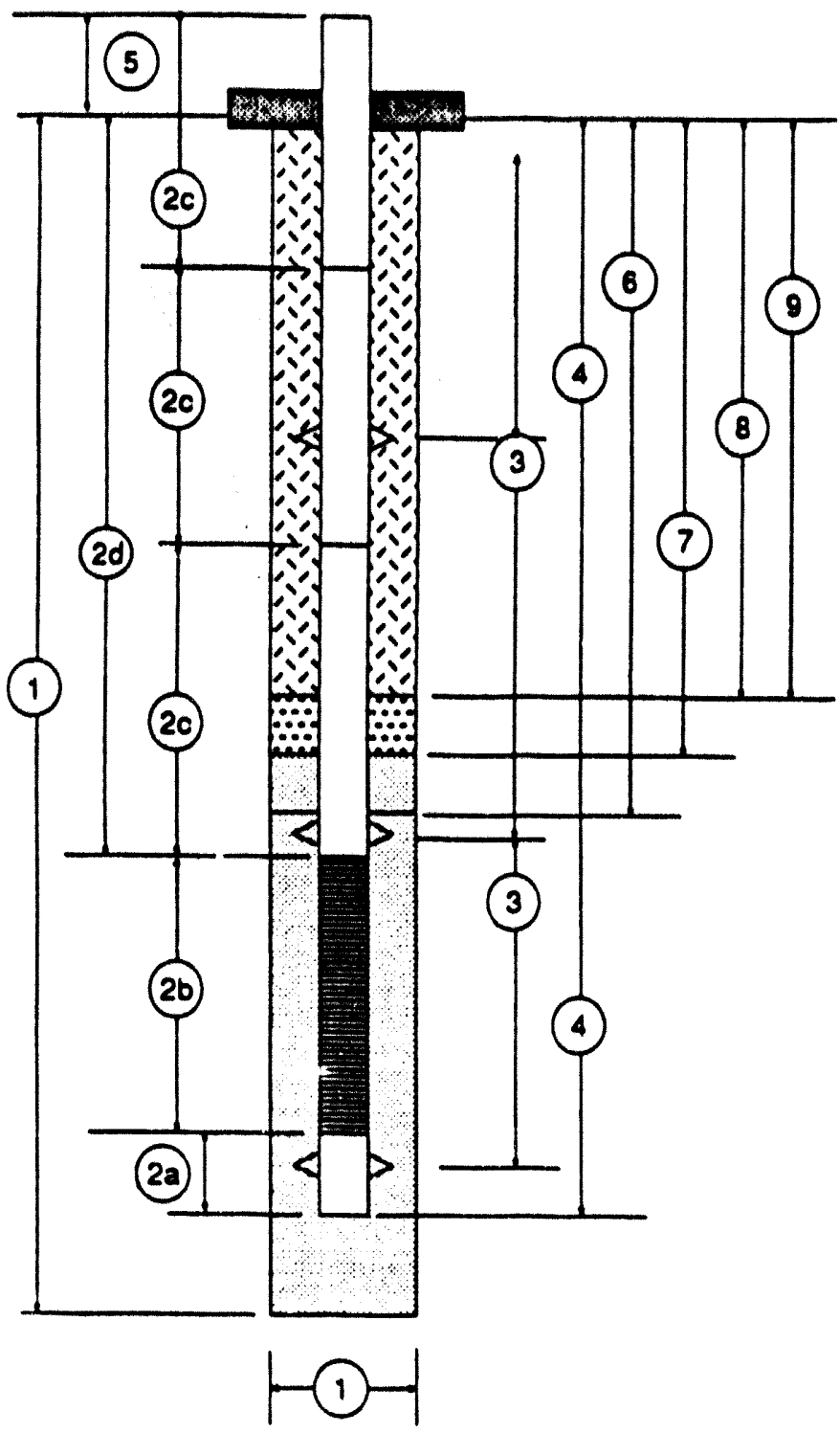

NOTE: ALL MEASUREMENTS

ARE FROM GROUND

SURFACE AT START

OF BORING - MEASUREMENTS

SANITARY SEAL ELEVATION

1) Total Drilled DepthHole Diameter $60.0^{\circ} / 81 / 4 "$

2) Casing/Scroen Tally (Measured to Nearest 0.01 Foot)

(a) Plug Longth

$0.02^{\circ}$

(b) Screen Longih 4.91

(c) Casing Joint Lengths (Moasured in Uphole Sequence from Top of Screen! \& $9.89^{\circ}$

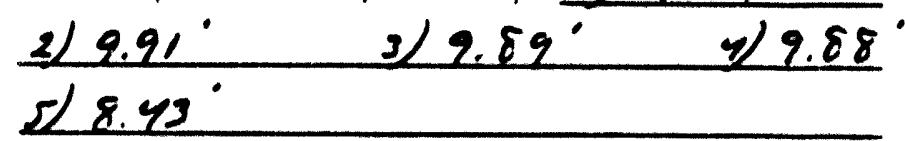

(d) Depth to Top of Screen

$48.00^{\prime}$

3) Depths to Centralizers

Not reguired

4) Total Depth of Installed Well

$52 \cdot 9^{\circ}$

5) Casing Stick Up (Standard 2.5 A.G.S.)

6) Depth 10 Top of Finer Pack $46.2^{\circ}$

7) Depth 10 Top of Fine Sand Seal Nat reguiced

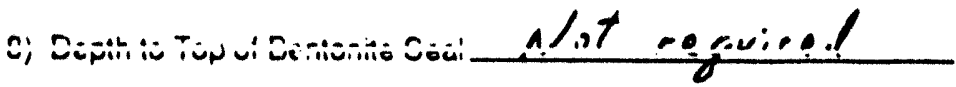

9) Thickness of Grout

$2.9^{\circ}$ 
DRILLING SUBCONTRACTOR EATC

DRILLER Bluke Cubit

DATE OF WELL INSTALLATION $12 / 03 / 92$

Tech. O.S.lco.name B.ll Joyle/ SEC frauhue

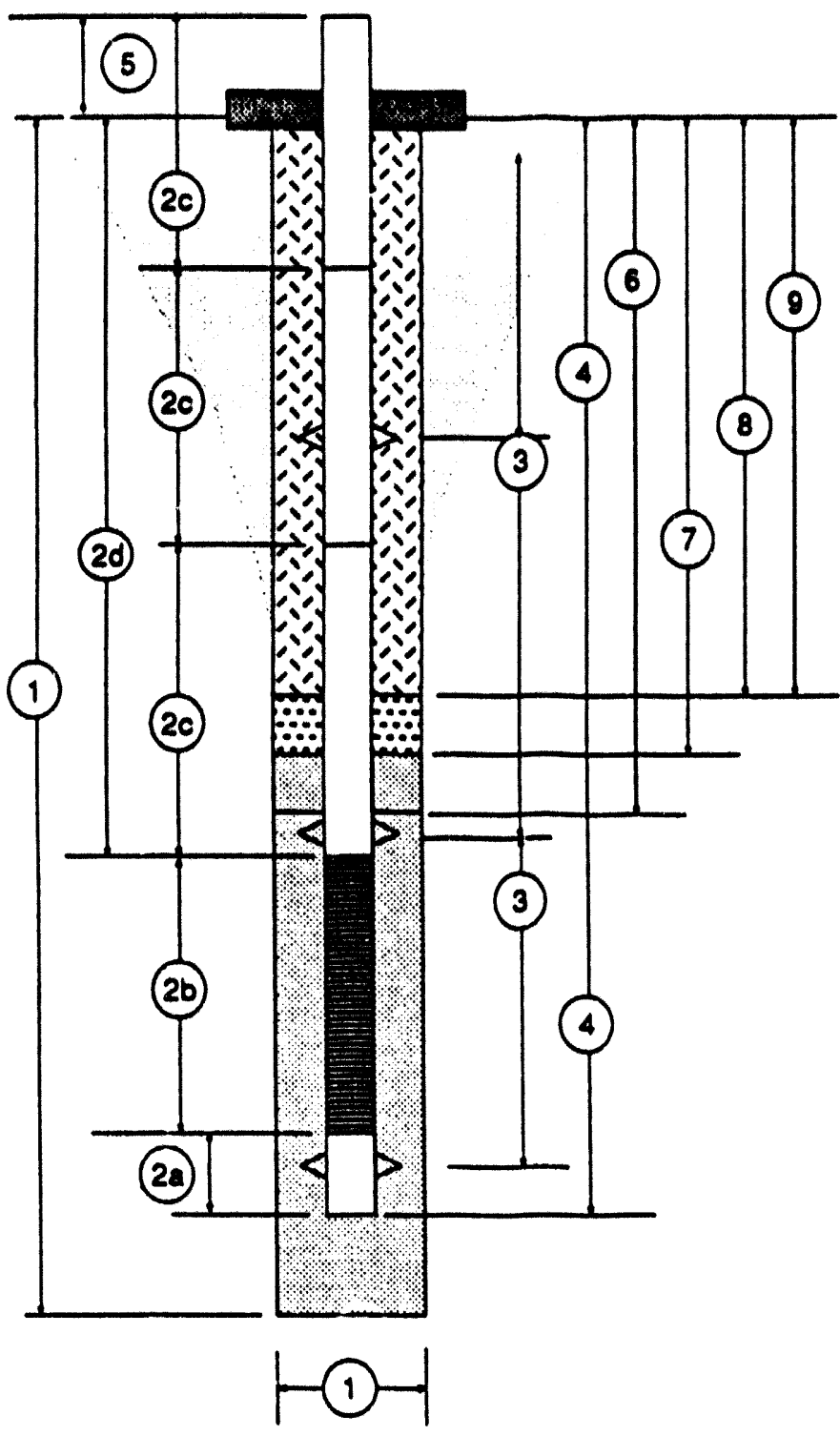

WELL NUMBER MHV-22C SRS COORDINATES

SANITARY SEAL ELEVATION

NOTE: ALL MEASUREMENTS

ARE FROM GROUND

SURFACE AT START

OF BORING - MEASUREMENTS

TO NEAREST 0.1 FOOT.

Secoad Geovt

2) Casing/Screen Tally (Measured to Nearest 0.01 Foot)

(a) Plug Length 0.02

(b) Scroen Length

$4.89^{\circ}$

(c) Casing Joint Longths (Moasured in Up. $29.88^{\circ}$

2) $9.87^{\circ} 3 / 25^{\circ}$

(d) Depth to Top of Screen

$28.00^{\circ}$

3) Depths to Centralizers Net reg viced

4) Total Depth of Installed Well

$32.9^{\circ}$

5) Casing Stick Up (Standard 2.5 A.G.S.) $1.7^{\circ}$

6) Depth to Top of Finer Pack

26.2

7) Depth 10 Top of Fine Sand Seal Not reguied

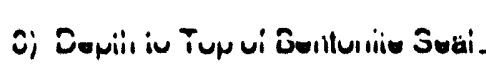

int resict

9) Thickness of Grout

$26.2^{\circ}$ 


\section{MONITORING WELL C.ONSTRUCTION DIAGRAM}

DRILLING SUBCONTRACTOR $\frac{\text { EATTC }}{\text { DRILLER } \frac{\text { B/ake CaG, }}{12 / 02 / 92}}$

WELL NUMBER MHV-2ZC

SRS COORDINATES

SANITARY SEAL ELEVATION

TECH. O.S./CO.NAME Bil/ Tijue/SEC Duauhue

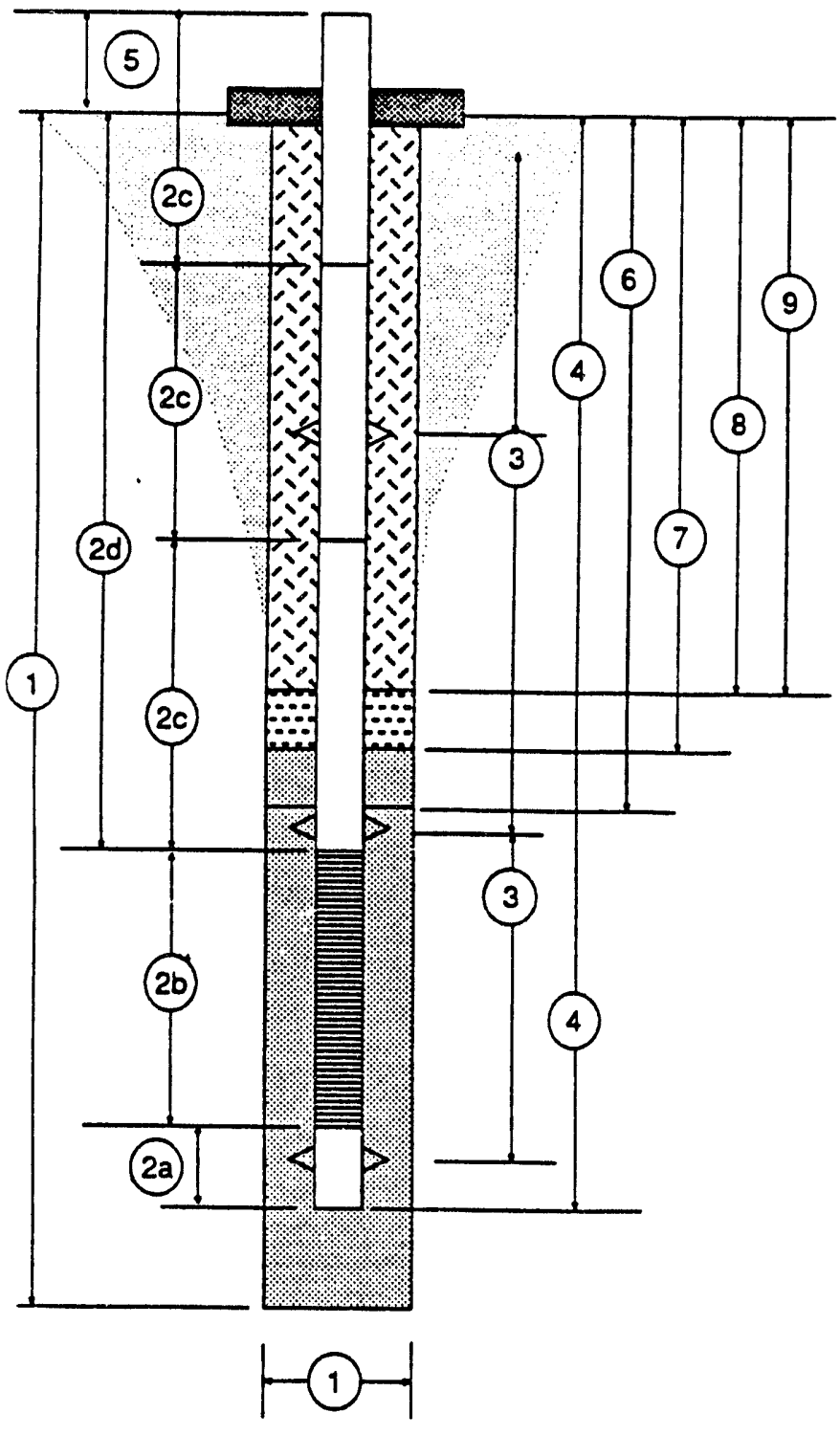

NOTE: ALL MEASUREMENTS

ARE FROM GROUND

SURFACE AT START

OF BORING - MEASUREMENTS

TO NEAREST 0.1 FOOT.

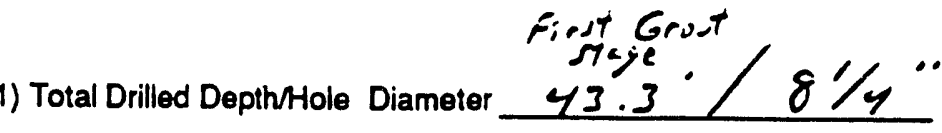

2) Casing/Screen Tally (Measured to Nearest 0.01 Foot)

(a) Plug Length

$0.02^{\circ}$

(b) Screen Length

$4.89^{\circ}$

(c) Casing Joint Lengths (Measured in Uphole Sequence From Top of Screen) 1) 9.91

2/ $9.91^{\circ}$ 3/ $9.92^{\circ}$ y/ 8.26

(d) Depth to Top of Screen

38.00

3) Depths to Centralizers Not reguired

4) Total Depth of Installed Well $42.9^{\circ}$

5) Casing Stick Up (Standard 2.5' A.G.S.) $1.7^{\prime}$

6) Depth to Top of Filter Pack 36.8 (natural soil)

7) Depth to Top of Fine Sand Seal Not reguired

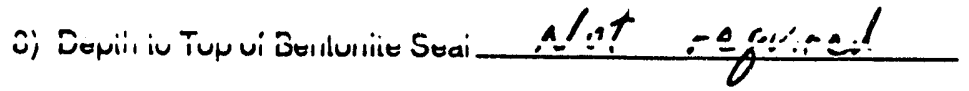

9) Thickness of Grout $1.9^{\circ}$ 
PAGE 1 OF 2

SEC DONOHUE

CLIENT WSRC

SUBJECT RF Heating Test - MHV - 2

$\mathrm{BY}^{-*}$ Bill Joyce DATE $12 / \mathrm{C3} / 42$ CHECKED BY

\section{CALCULATION SHEET}

LOCATION

SRS

JOB NO. G. 4346

Piezoneter Well Construction Diagram DATE
Bottom

Casing
From. $\quad M H V-22$
Frum

Grovad

Surface
Gravel Pack

Based on Practical

Application in the Field of bag Coarse Sand $=1.2 \mathrm{ft}^{3}$

bag Groct $=0.6 \mathrm{ft}^{3}$

Sas Mixad $=1.3 \mathrm{ft}^{3}$

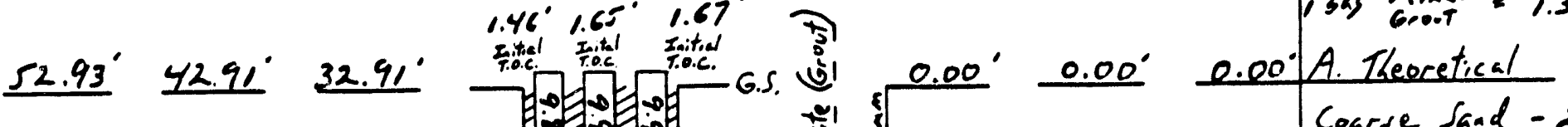

$44.5^{\circ} \quad 34.65^{\prime} \quad 24.66^{\circ}$

$34.62^{\circ} \quad 24.73^{\circ} \quad 14.79^{\circ}$

$24.73^{\circ} \quad 14.82^{\circ} \frac{4.91^{\circ}}{0.02}$

$14.82^{\circ}, 4.91^{\circ} \quad \frac{0.02}{\left(0.00^{\circ}\right.}$

$4.93^{\circ} \frac{0.02^{\circ}}{\left(0.00^{\prime}\right)}$

$\frac{0.02^{\prime}}{(0.00)}$

Coarse Sand - O Nill

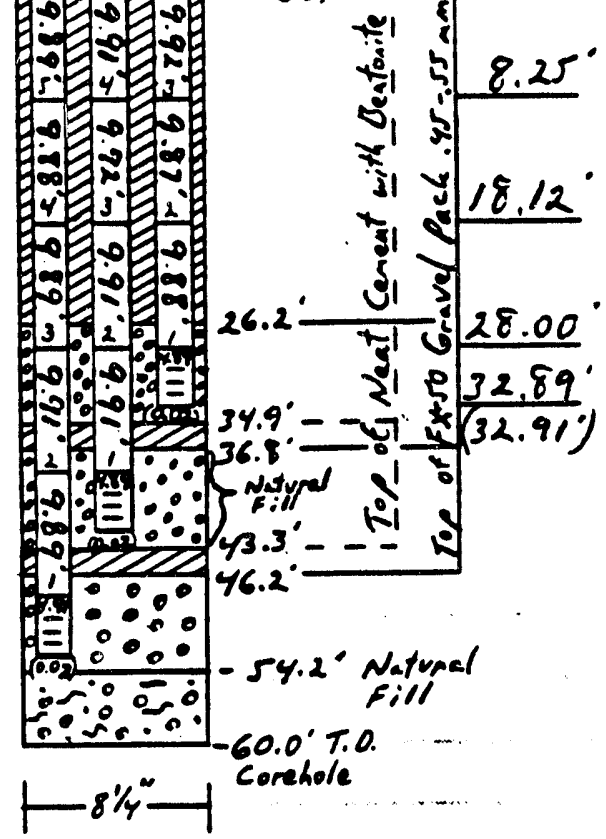

$+14$
Coarse Sand - $2.3 \mathrm{bl}$ :

Grout -0.6 bags

Actral

Coarse Sand - 3.5 ba;

Grout - 0.7 bags

$28.09^{\circ} \quad 28.20$

$38.00^{\circ} \quad 38.11^{\circ}$

$42.89^{\circ}$

$(42.9 !)$

$\frac{48.00^{\circ}}{\frac{52.91^{\circ}}{\left(52.93^{\circ}\right)}}$

Grout - $0.7 \mathrm{bags}$

c. Theoretical

Coarse Sand-2.36ay

Grout - 7.2 bags

Actual

Coarse Sand - 2.25 bs

Grout - 5.7 bags 


$$
\text { PAGE } 2 \text { of } 2
$$

SEC DONAHUE

CLIENT WSRC

SUBJECT RE Heating Test - MHV - 22
CALCULATION SHEET

LOCATION SR S

JOB NO. G4346

Piezometers Casing Tally

DATE

Screen / plug

Tally

A

$\frac{\text { Casing }}{1 " \text { PvC }}$

Schedule yo

Tri-Loc Threads

Flush

Timco Brand

$\frac{\text { Screen }}{\text { "PVC }}$

\begin{tabular}{l|l|l|}
\hline \multirow{4}{*}{$4.91^{\circ}$} & & $0.09^{\circ}$ \\
\cline { 2 - 3 } & $\equiv$ & $4.68^{\circ}$ \\
& $\equiv$ & \\
\hline & & $0.14^{\circ}$ \\
\hline $0.02^{\circ}$ & plug & $0.02^{\circ}$ \\
\hline
\end{tabular}

slotted

0.010 in. slot

Flush

Tri-Loc Threads

Times Grand

Sump

Not required

plug

"pvc

Schedule yo

Tri-Luc Threads

Flush

Timco Brand $\beta$

\begin{tabular}{l|l|l|}
\hline & & $0.08^{\circ}$ \\
\cline { 2 - 3 } $4.89^{\circ}$ & $=$ & \\
& $=$ & $4.68^{\circ}$ \\
& $=$ \\
\cline { 2 - 3 } & & $0.13^{\circ}$ \\
\hline $0.02^{\circ}$ & plug & $0.02^{\circ}$ \\
\hline
\end{tabular}

$c$

\begin{tabular}{l|l|l|}
\hline & & $0.09^{\circ}$ \\
& $=$ \\
$4.89^{\circ}$ & $\equiv$ & $4.67^{\circ}$ \\
& $\equiv$ & \\
& $=$ & $0.13^{\circ}$ \\
\cline { 2 - 3 } & & $0.02^{\circ}$ \\
\hline $0.02^{\circ}$ & $p \log$ & 0.0 \\
\hline
\end{tabular}


URILLING SUBCONTRACTOR EMTC DRILLER Stive Riesi DATE OF WELL INSTALLATION $1 / 21 / 93$ TECH. O.s.lco.name Bill Juyce/SEC Donohue

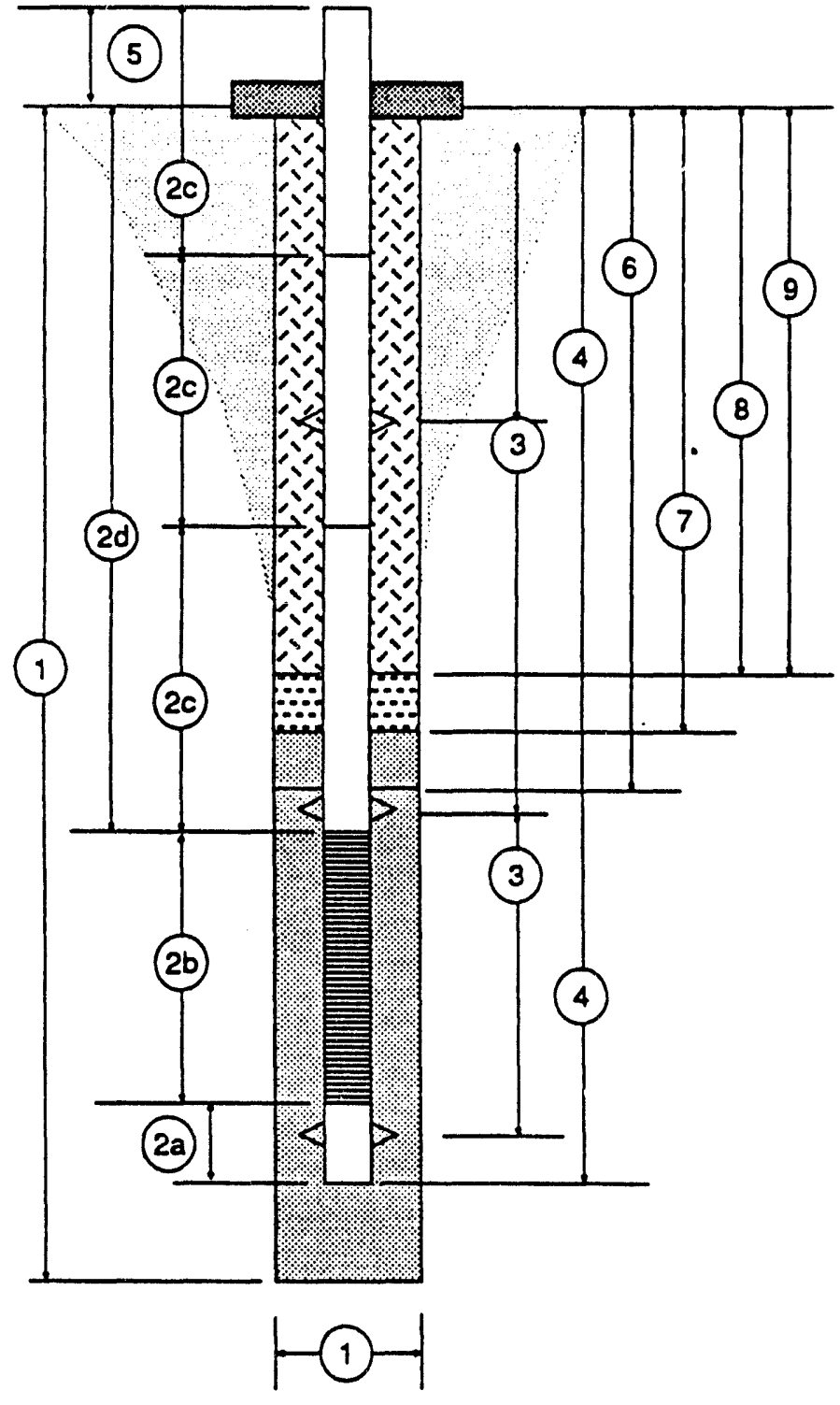

WELL NUMBER MHV-22T

SRS COORDINATES

SANITARY SEAL ELEVATION

NOTE: ALL MEASUREMENTS

ARE FROM GROUND

SURFACE AT START

OF BORING - MEASUREMENTS

TO NEAREST 0.1 FOOT.

Fiber Optics Temperature Scusors

1) Total Drilled Depth/Hole Diameter $63.0^{\circ} 8$ "

2) Casing/Screen Tally (Measured to Nearest 0.01 Foot)

(a) Sump \& Plug Length

Not reguired

(b) Screen Length Not reguired

(c) Casing Joint Lengths (Measured in Uphole Sequence From Ton of Screen) I 10.12 including threads $210.00^{\circ}$ 3) $10.00^{\circ}$ 4) $10.00^{\circ}$ 5) $10.00^{\circ}$ 6) $4.88^{\circ}$

(d) Depth to Sepoforseron Fiber optics: $28.00^{\circ}, 33.00^{\circ}$, $38.00^{\circ}$. 43.00\% $48.00^{\circ}$ and $53.00^{\circ}$. Thermocouples: $28.00^{\circ}, 38.00^{\circ}$ and $48.00^{\circ}$

3) Depths to Centralizers Not reguired

4) Total Depth of Installed Well $55.0^{\circ}$

5) Casing Stick Up (Standard 2.5' A.G.S.) 5.1

6) Depth to Top of Filter Pack 9.6

7) Depth to Top of Fine Sand Seal Not reguired

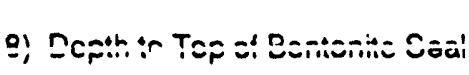
$\therefore$ if ra.iral

9) Thickness of Grout 9.6 


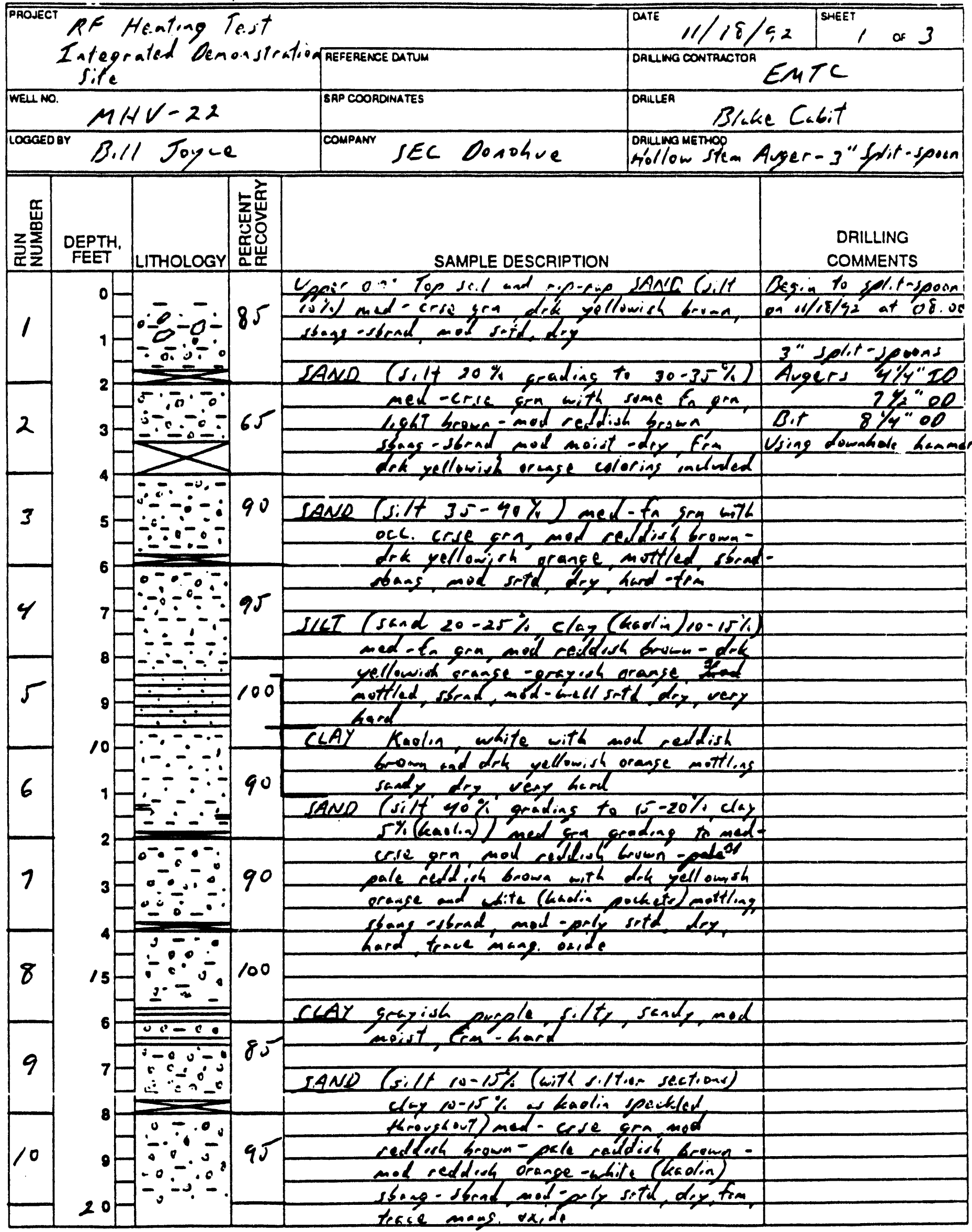


FIELD GEOLOGIC LOG

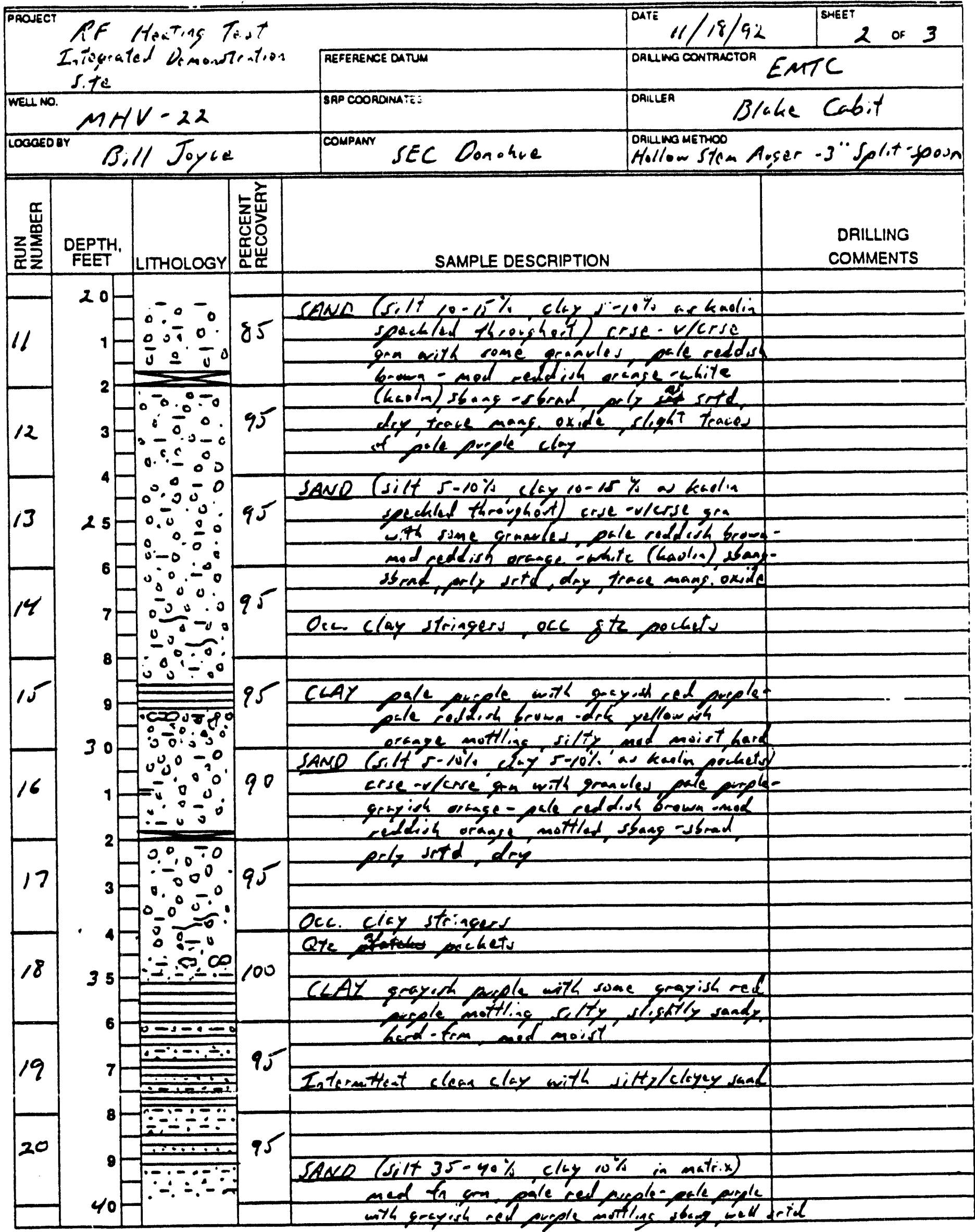


FIELD GEOLOGIC LOG

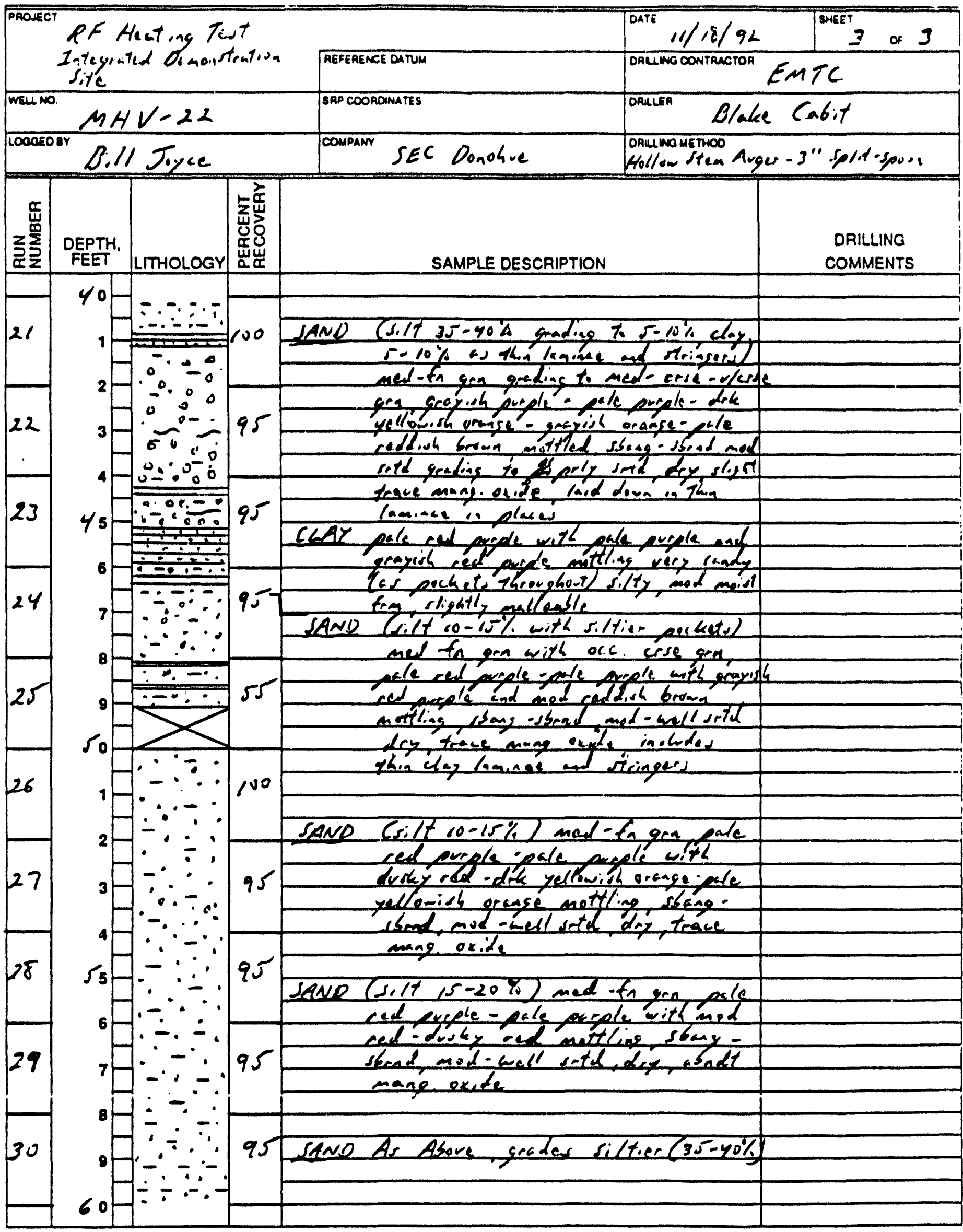


DRILLING SUBCONTRACTOR EMTC

DRILLER

Biake Cá,

DATE OF WELL INSTALLATION $11 / 20 / 92$
WELL NUMBER $M H V-23 A$ SRS COORDINATES TECH. O.S.ICO. Name Bill Joyce/SEC Do.1shue

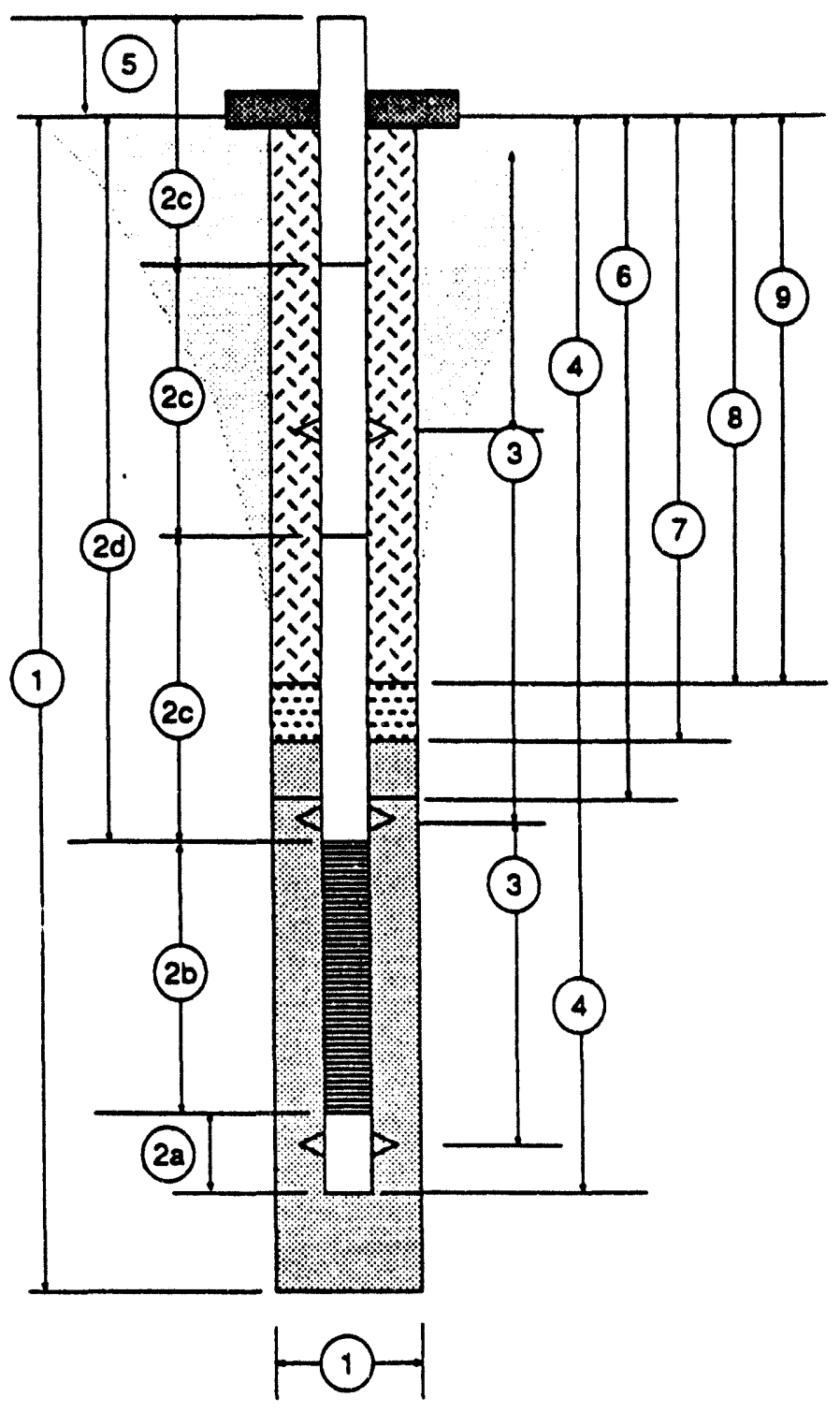

NOTE: ALL MEASUREMENTS

ARE FROM GROUND

SURFACE AT START

OF BORING - MEASUREMENTS

TO NEAREST 0.1 FOOT.

1) Total Drilled Depth/Hole Diameter $60.0^{\circ} / 814^{\circ}$

2) Casing/Screen Tally (Morsured to Nearest 0.01 Foot)

(a) Suespe Plug Longth $\quad 0.03$

(b) Screen Length 4.89

(c) Casing Joint Lengths (Measured in Uphole Sequence From Top of Screen) 2) $9.90^{\circ}$ 3) $9.90^{\circ}$ 5) $8.43^{\circ}$

(d) Depth to Top of Screen

$48.00^{\circ}$

3) Depths to Centralizers

Not reguired

4) Total Depth of Installed Well

52.9

5) Casing Stick Up (Standard 2.5' A.G.S.) $1.2^{\circ}$

6) Depth to Top of Fitter Pack $46.5^{\circ}$

7) Depth to Top of Fine Sand Seal Not regvired

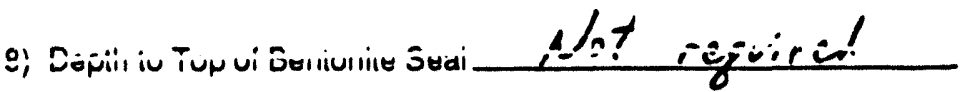
9) Thickness of Grout $2.0^{\circ}$ 
DRILLING SUBCONTRACTOR ENTC DRILLER Pluke Cab,T DATE OF WELL INSTALLATION $12 / 01 / 92$ TECH. O.S.ICO. NAME B.ll Joyce / JEC Donohue

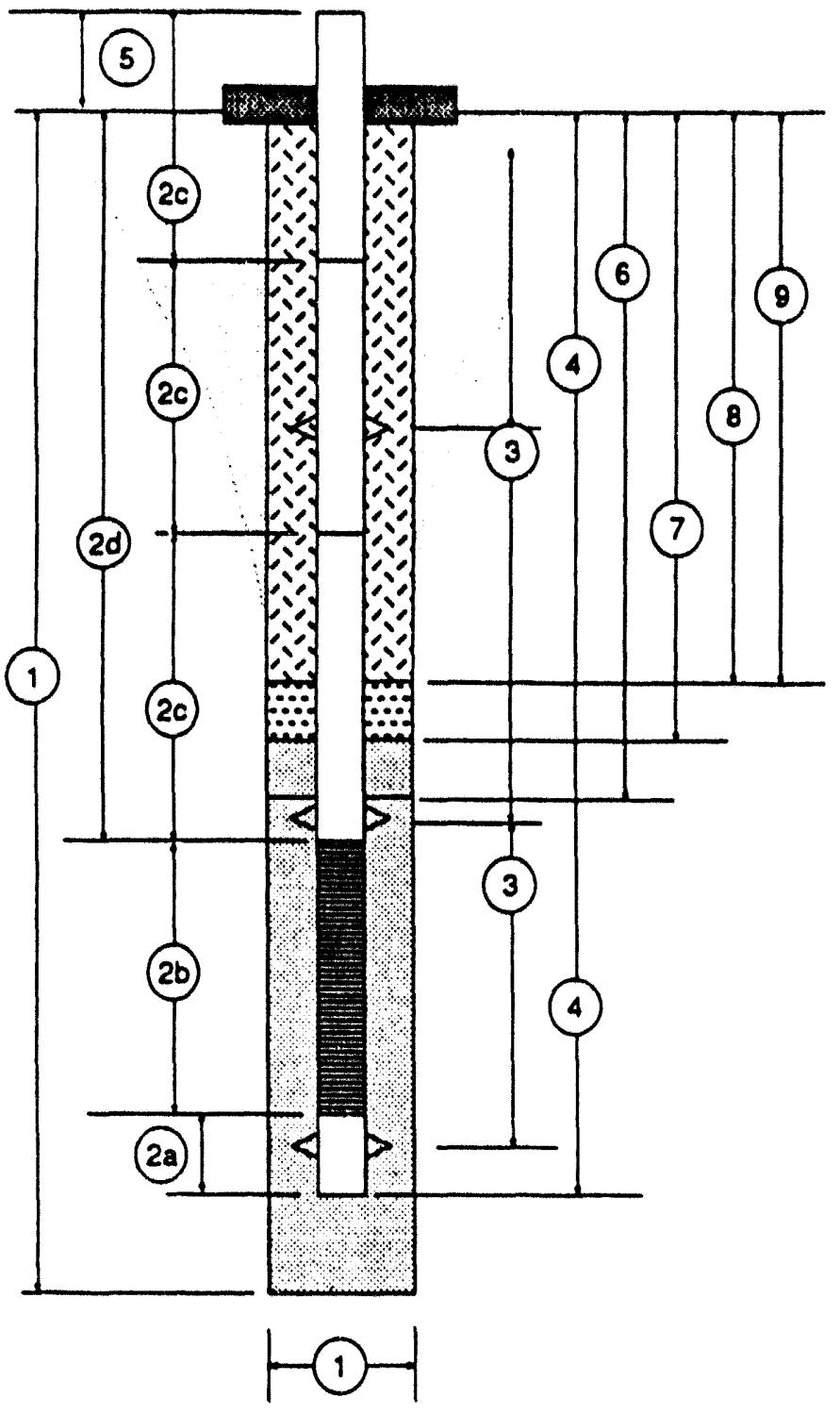

WELL NUMBER MAV- $23 B$

SRS COORDINATES

SANITAAY SEAL ELEVATION

NOTE: ALL MEASUREMENTS

ARE FROM GROUND

SUAFACE AT START

OF BORING - MEASUREMENTS

TO NEAREST 0.1 FOOT.

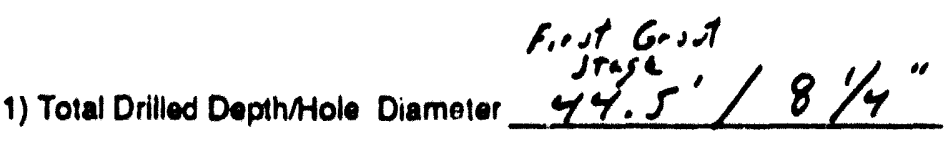

2) Casing/Screon Tally (Measured to Ne zrest 0.01 Foot)

(a) Plug Longth $0.02^{\circ}$

(b) Screen Length $\quad 4.89^{\circ}$

(c) Casing Joint Lengths (Measured in Up. 2/ $9.90^{\circ} \quad 3 / 9.90^{\circ} 4 / 8.31^{\circ}$

(d) Depth to Top of Screen

$38.00^{\circ}$

3) Depths to Centralizers Nct reguired

4) Total Depth of Installed Woll

$42.9^{\circ}$

5) Casing Stick Up (Standard 2.5' A.G.S.)

6) Depth to Top of Filler Pack

36.5

7) Depth 10 Top of Fine Sand Seal Net refviced

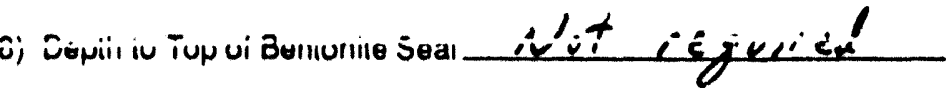

9) Thickness of Groul 1.8 
DRILLING SUBCONTRACTOR EMTC DRILLER $31 . k \mathrm{C}$ Cakit

DATE OF WELL INSTALLATION $12 / 13,9,9$ TECH. O.S.ICO. name B.ll Tuyge/SEC Dourdue

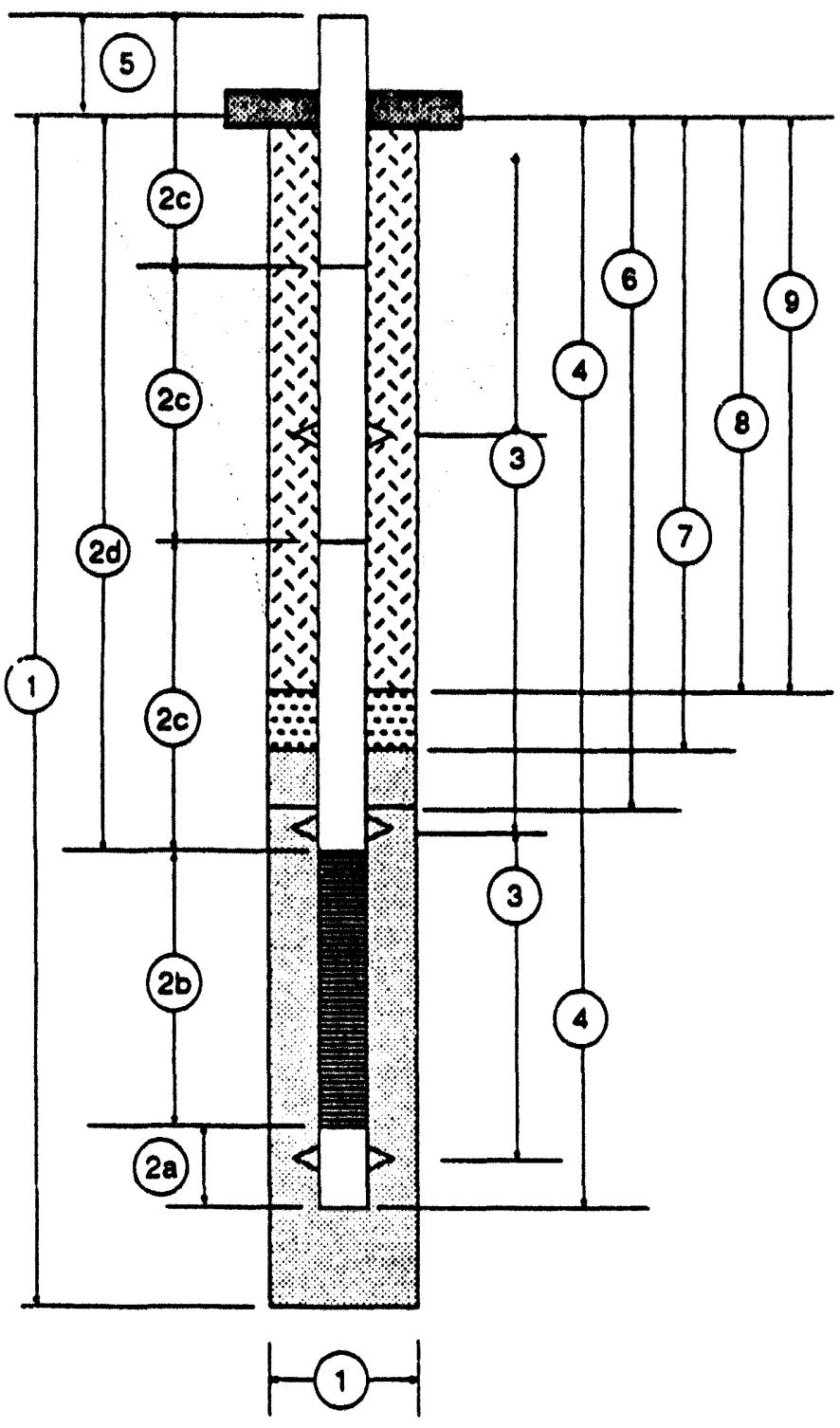

WELL NUMBER MHV-23C SRS COORDINATES

SANITARY SEAL ELEVATION
NOTE: ALL MEASUREMENTS

ARE FROM GROUND

SURFACE AT START

OF BORING - MEASUREMENTS

TO NEAREST 0.1 FOOT.

1) Total Drilled DepthMole Diamelor $\begin{gathered}\text { Second Gro.t } \\ \text { sioge } \\ 34.2 \%\end{gathered}$

2) Casing/Screen Tally (Measured to Nearest 0.01 Foot)

(a) Plug Length $0.04^{\circ}$

(b) Screen Length $4.89^{\circ}$

(c) Casing Joint Longths (Measured in Up hole Sequence From Top of Scieen) 1) $9.88^{\circ}$ 2) $9.87^{\circ} 3 / 8.25^{\circ}$

(d) Depth to Top of Screen

$28.00^{\circ}$

3) Depths 10 Contralizers Not cegreced

4) Total Depith of Installed Woll

32.9

5) Casing Stick Up (Standard 2.5' A.G.S.) $1.63^{\prime}$

6) Depth to Top of Finer Pack $25.9^{\circ}$

7) Depth to Top of Fine Sand Seal Net ceguired

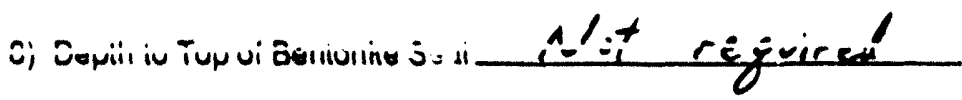

9) Thickness of Grout 25.9 
rbag lis - paoso roge - pos osones $1>726$ $5 \log 7 \cdot 6-10009$ ing e.z-pus asonos

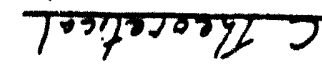
rbog L.0 - p0.19 lap s.t - pros asovos Llog.9.0- tioso Sogh.t - yos orstos $.11 .85 .003 \varepsilon$

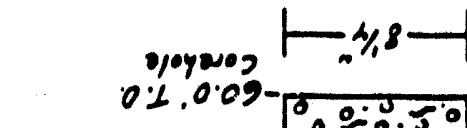
$1+1$

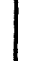
Tiगfordy

rlog 6.0 - jnoगg

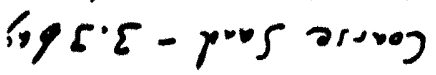

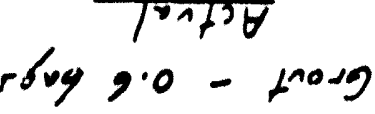
ighiz-p.y ardios $\overline{77 \cdot 82} .7187$ $.68^{2} 25$

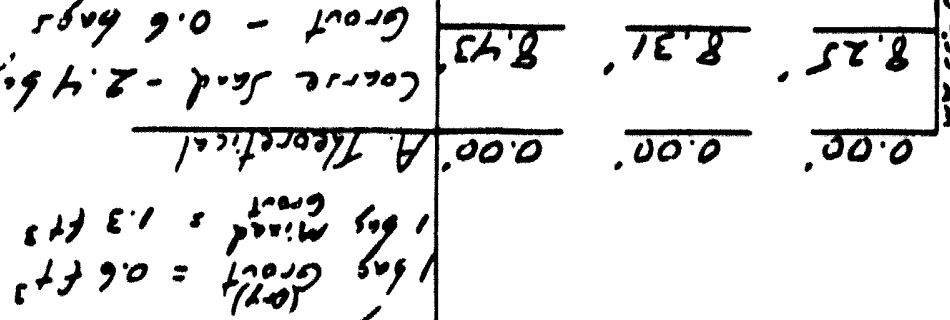

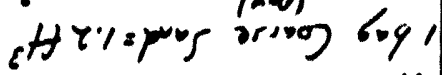

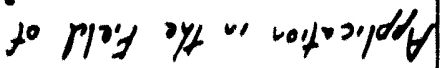
priforey wo pory

$$
\begin{aligned}
& \text { गयन्तर } \\
& \text { prasesg } \\
& \text { worg }
\end{aligned}
$$

$$
0.0 .09-
$$

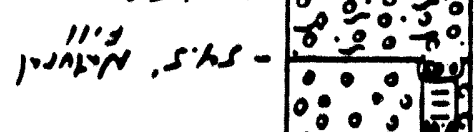

s.9h (1) iv $\frac{.00 \cdot 0)}{70.0} . \overline{76 \%}$ $\frac{(.00 \cdot 0)}{1000} \cdot 76 \% \quad .78 \%$ . $56 \mathrm{~h} . \overline{0 s h 1}, \overline{16 h \mathrm{r}}$ $. \overline{18 \% 1} . \overline{0 L h \overline{1}} . \overline{19 \mathrm{hE}}$ $. \overline{89 h t} . \overline{09 h \varepsilon} . \overline{6 h \% h}$

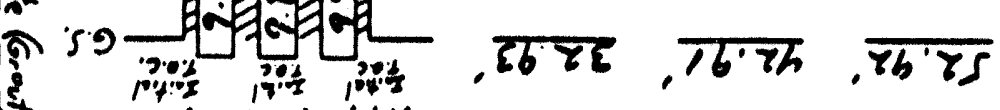
(4)
T.1\%5

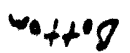
woy

$\exists \perp \forall 0$

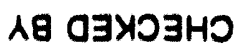

NOIL $\forall 07$

$7 \times 5 M$

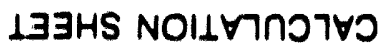




$$
\text { AnE_- } 2 \text { a } 2
$$

SEC DONAHUE.

CALCULATION SHEET

CLIENT W WSRC LOCATION SET JOB NO. G4346

subJect RE Heating Test - MHV - 23 Pezometers Canine Tally By Bill Joyce DATE $12 / 03 / 4,2$ CHECKED BY DATE

$\frac{\text { Casing }}{\text { Tally }}$

2) $9.90^{\circ}$

3) $9.90^{\circ}$

4) $9.88^{\circ}$

5) $\frac{9.90^{\circ}}{49.47^{\circ}}$

$-48.00^{\circ}$ Top of Casing $1.49^{\circ}$ stich -up

$\frac{B}{9.89^{\circ}} \cdot \frac{B}{38.0^{\circ}-43.0^{\circ}}$

2) $9.90^{\circ}$

3) $9.90^{\circ}$

4) $\frac{9.88^{\circ}}{39.57^{\circ}}$

$\frac{-38.00^{\circ}}{1.57}$. Top of Cawing $1.57^{\circ}$ stickup

1) $\frac{c}{9.88^{\circ}} \quad \frac{c}{28.0^{\circ}-33.0^{\circ}}$

2) $9.87^{\circ}$

3) $\frac{9.88^{\circ}}{29.63^{\circ}}$

$\frac{-2800^{\circ}}{1.63^{\circ} \text { 'Top of Casting - op }}$
Materials

List $(A, B C)$

$\frac{\text { Casing }}{1 " \text { Pvc }}$

schedule yo

Tri-Loc Threads

Flush

Timco Brand

$\frac{\text { Screen }}{1 \text { PVC }}$

slotted

0.010 in. slot

Flush

Tri-Loc Threads

Times Brand

Sues

Not required

plug

Schedule yo

Tri-Luc Treads

Flush

Tinct Grand
Screen / ploy

Tally

A

\begin{tabular}{l|l|l|}
\hline \multirow{4}{*}{$4.89^{\circ}$} & $=$ & $0.08^{\circ}$ \\
\cline { 2 - 3 } & $=4.68^{\circ}$ \\
& $=$ \\
\cline { 2 - 3 } & & $0.13^{\circ}$ \\
\hline $0.03^{\circ}$ & plug & $0.03^{\circ}$ \\
\hline
\end{tabular}

$\beta$

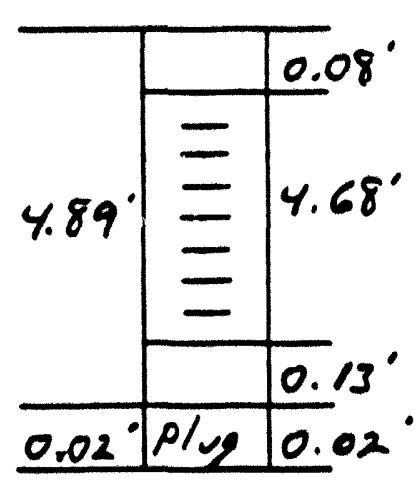

$c$

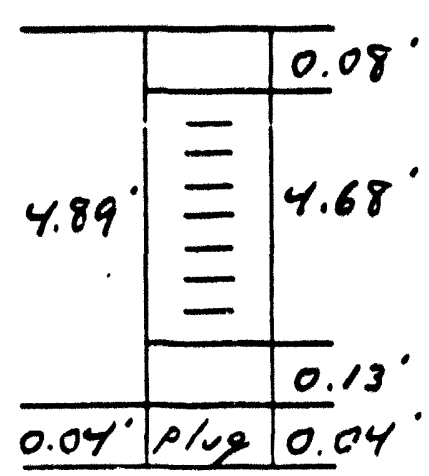


DRILLING SUBCONTRACTOR EMT:

DRILLER Steve Reesi

DATE OF IVELL INSTALLATION

$1 / 21 / 93$

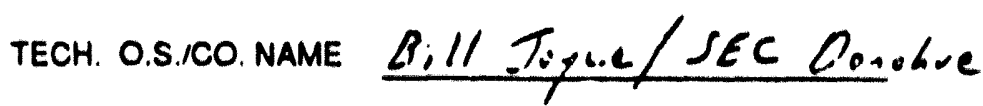

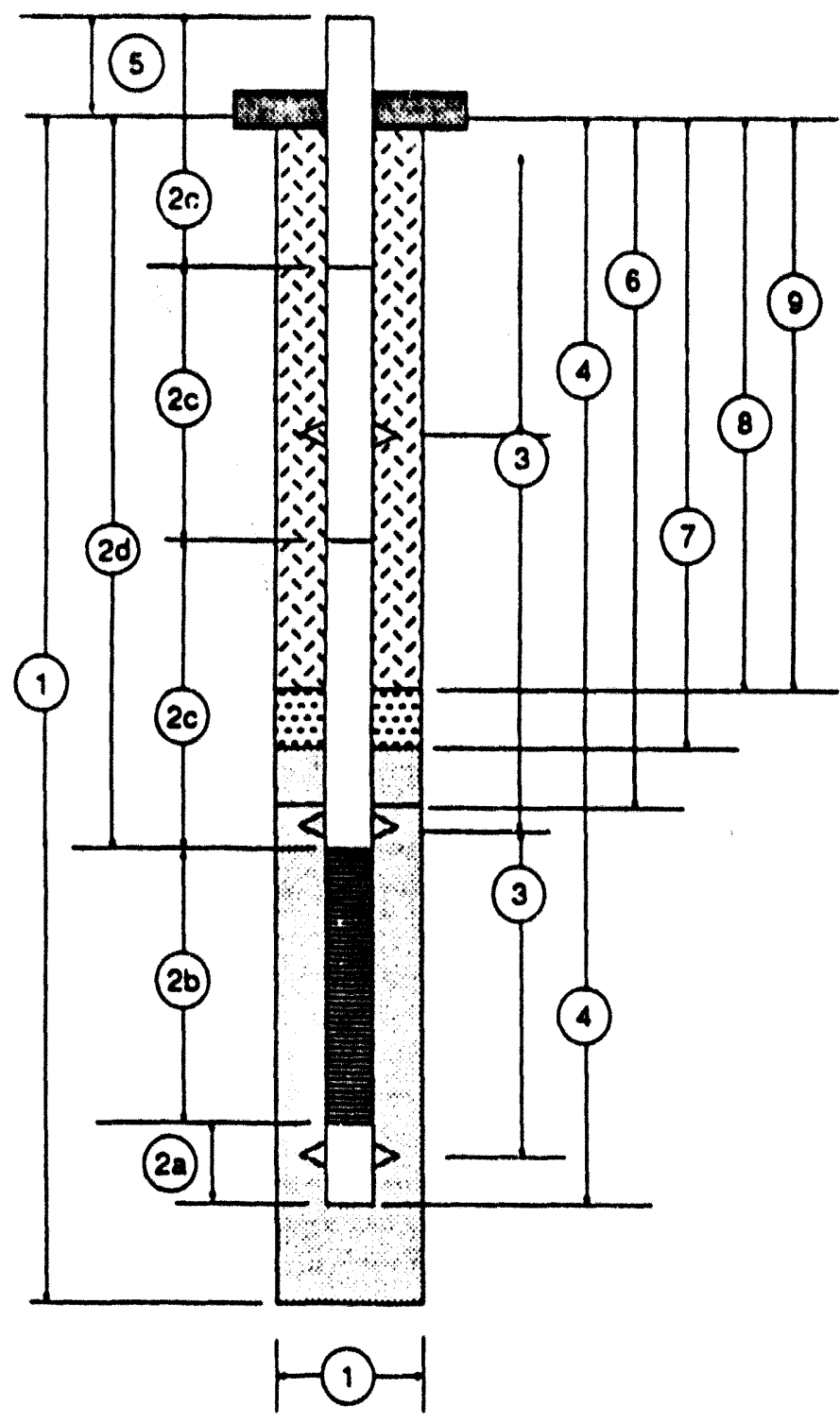

WELL NUMBEA $M H V \cdot 237$ SAS COORDINATES SANITARY SEAL ELEVATION

NOTE: ALL MEASUREMENTS ARE FROM GROUND SUPFACE AT START OF BORING - MEASUREMENTS TO NEAREST $0.1 \mathrm{FOC}^{-}$ Fiber Gptics Temperatore Sossors 1) Total Drilled DepthAlole Diameter $63.0^{\circ} / 8 \%{ }^{\circ}$ 2) Casing/Screon Tally (Moasured to Nearest 0.01 Foot)

(a) Sump \& Plug Longth Nut reguired

(B) Scroen Longth Not cegeired (c) Casing Joint Lengths (Moasurad in Up-
holo Sequenco From Top of Screon) $10.12^{\circ}$ including thread is $10.00^{\circ}$ \$/10.00 $\quad 4 / 10.00^{\circ} \quad 5 / 10.00^{\circ}$ 6) $4.88^{\circ}$

(d) Dopth 10 Sepopors

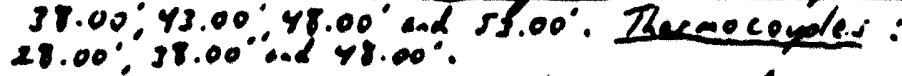

3) Dopths 10 Centralizers alet pegrired

4) Total Depth of Installed Well $55.0^{\circ}$

5) Casing Stick Up (Standard 2.5' A.G.S.) _

6) Depth 10 Top of Finer Pack $9.1^{\prime}$

7) Depth 10 Top of Fine Sand Seal Not regured

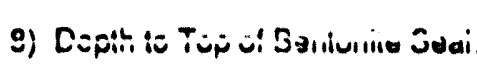
itt igyicen

9) Thickness of Grout $9.1^{\circ}$ 
FIELD GEOLOGIC LOG

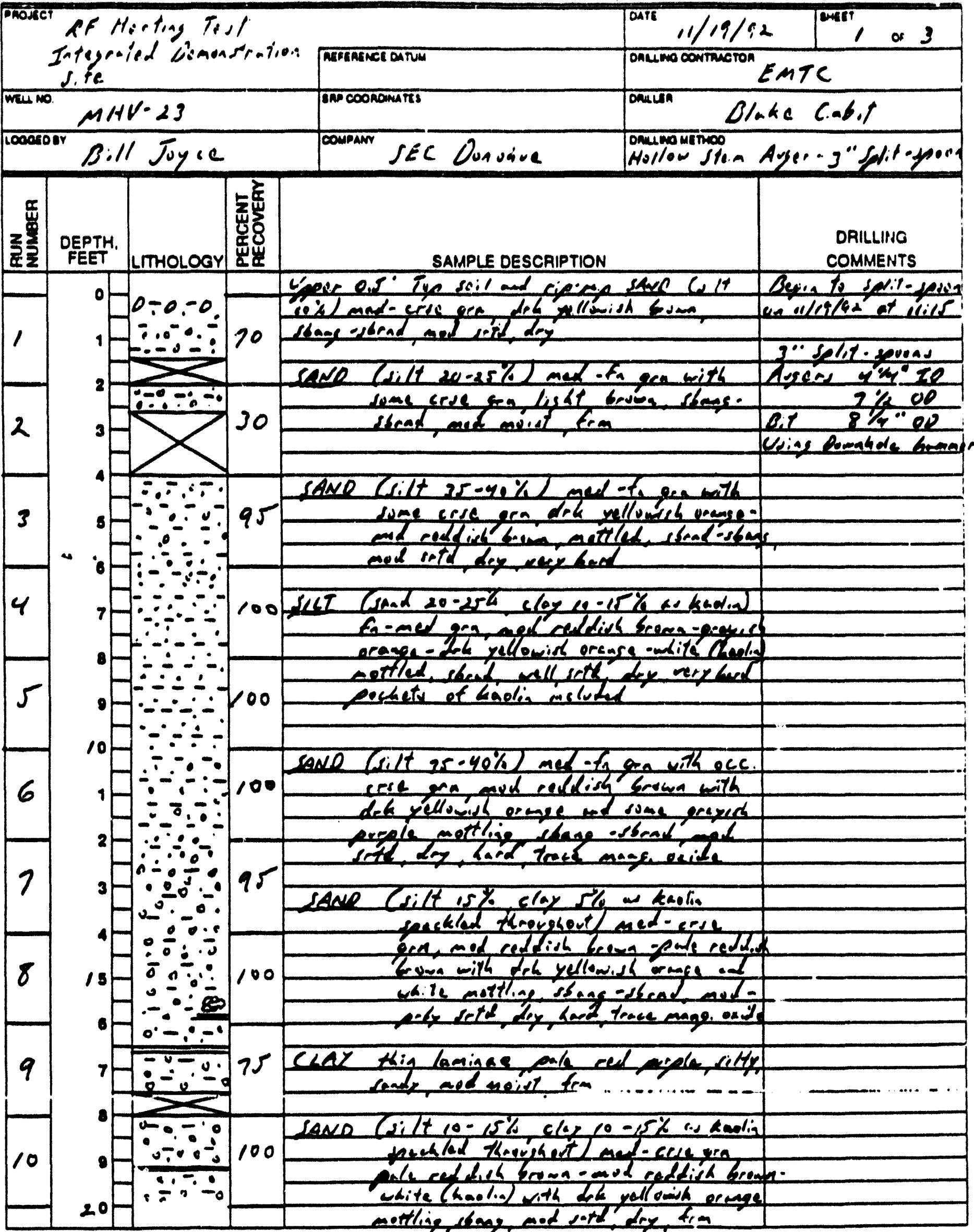


FIELD GEOLOGIC LOG

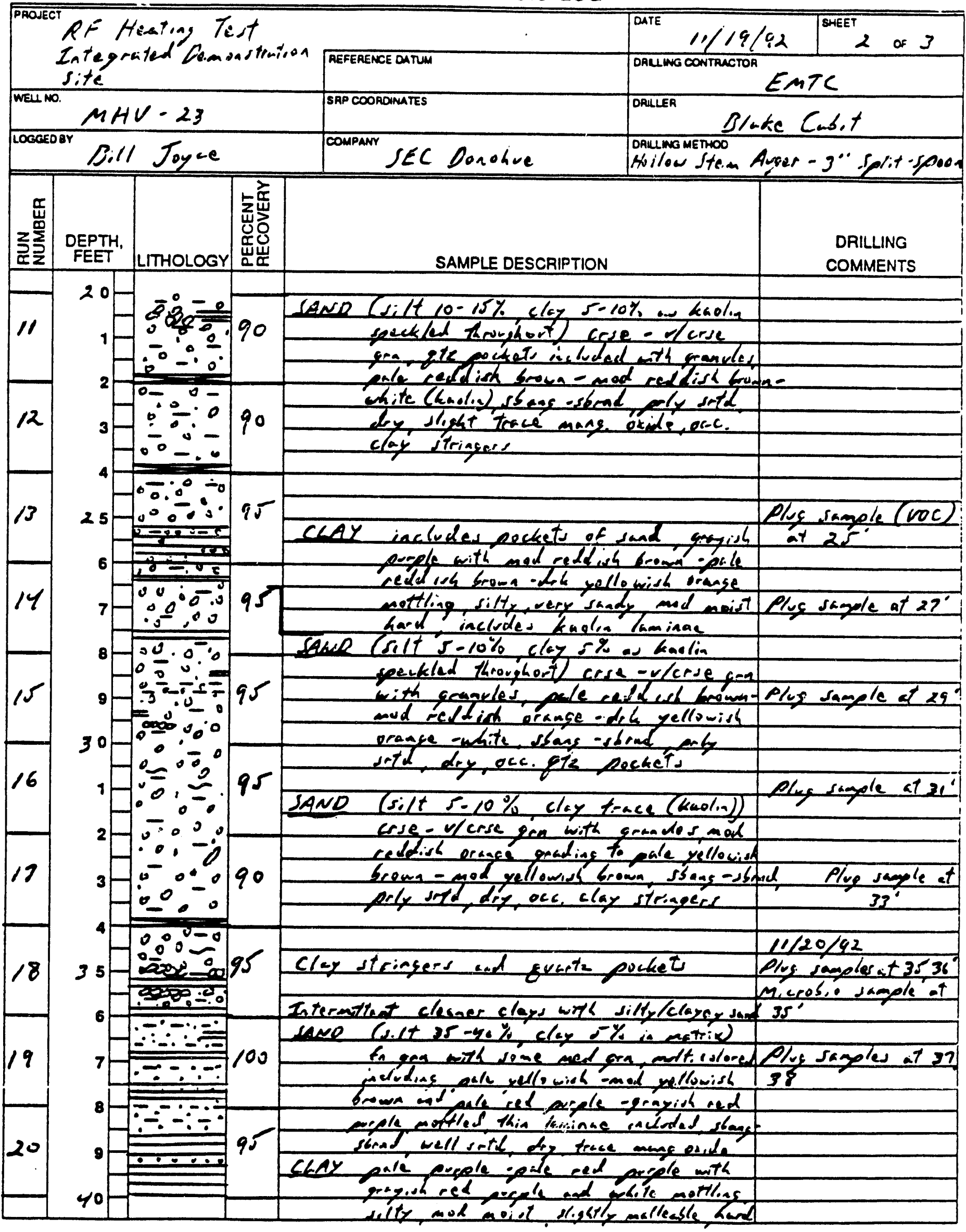


OSR 30.9

FIELD GEOLOGIC LOG

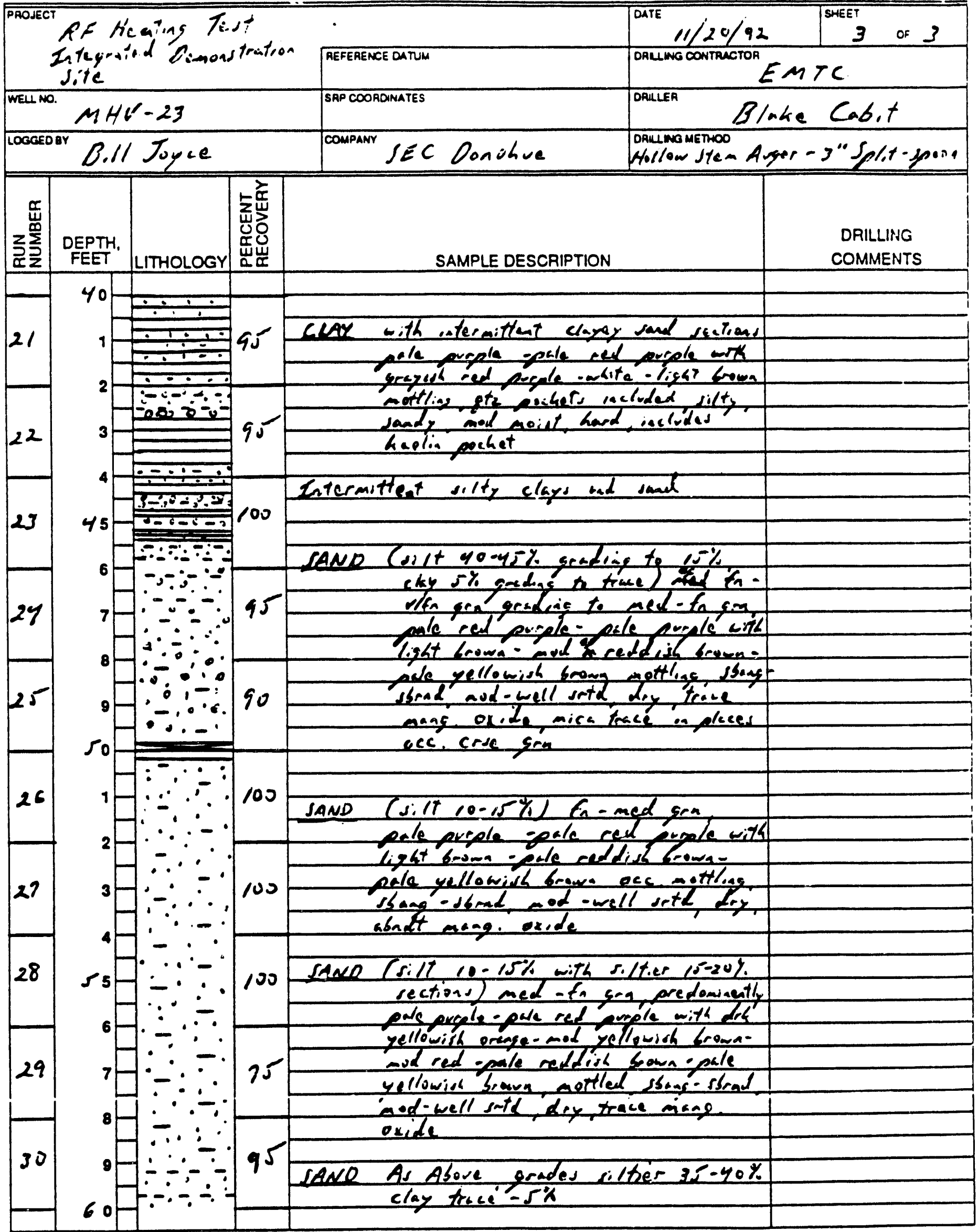




\section{MONITORING WELL CONSTRUCTION DIAGRAM}

DRILLING SUBCONTRACTOR EMTC.

DRILLER

Blake Cabit

DATE OF WELL INSTALLATION $11 / 17 / 92$

TECH. O.S.ICo.name Dill Joyce/SEC Donukue

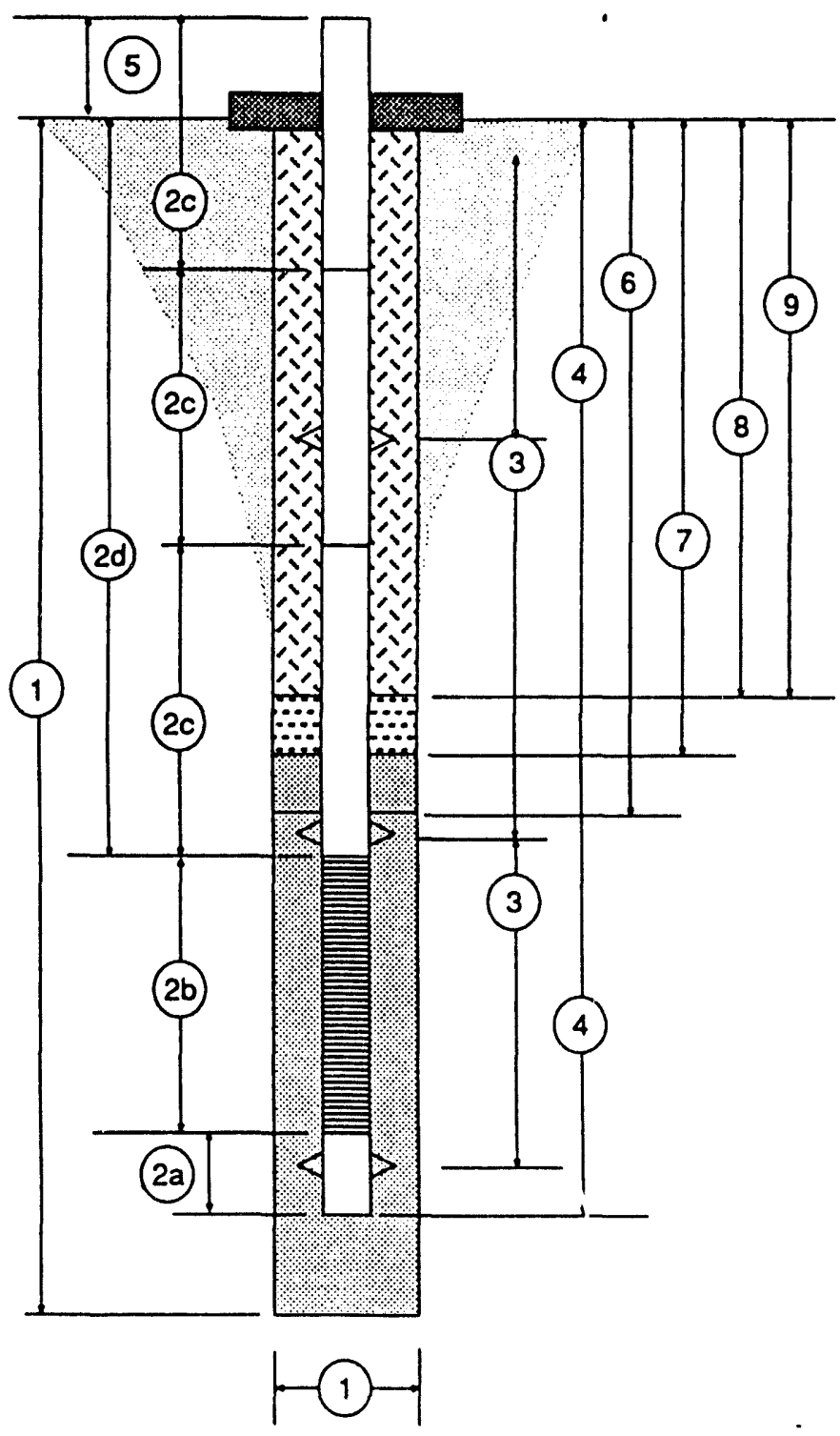

WELL NUMBER_MHV-24A

SRS COORDINATES

SANITARY SEAL ELEVATION
NOTE: ALL MEASUREMENTS

ARE FROM GROUND SURFACE AT START OF BORING - MEASUREMENTS TO NEAREST 0.1 FOOT.

1) Total Drilled Depth/Hole Diameter $60.0^{\prime} / 81 / 4 \%$

2) Casing/Screen Tally (Measured to Nearest 0.01 Foot)

(a) Plug Length $0.03^{\circ}$

(b) Screen Length $4.90^{\circ}$

(c) Casing Joint Lengths (Measured in Up- 1) $9.87^{\circ}$
hole Sequence From Top of Screen) 2) $9.87^{\circ}$ 3) 9.90 4) $9.89^{\circ}$ 5) $8.47^{\circ}$

(d) Depth to Top of Screen_ $48.00^{\prime}$

3) Depths to Centralizers Not ceguired

4) Total Depth of Installed Well

52.9

5) Casing Stick Up (Standard 2.5' A.G.S.) _ 1.4

6) Depth to Top of Filter Pack $46.3^{\prime}$

7) Depth to Top of Fine Sand Seal __ Not reguired

8) Depth to Top of Bentonite Seal__Not regvired

9) Thickness of Grout 1.9 
DRILLING SUBCONTRACTOR EMTC DRILLER Blake Cabit

DATE OF WELL INSTÁLLATION $11 / 19 / 92$ TECH. O.S.ICO. NAME Bill Joyce/SEC Donohue

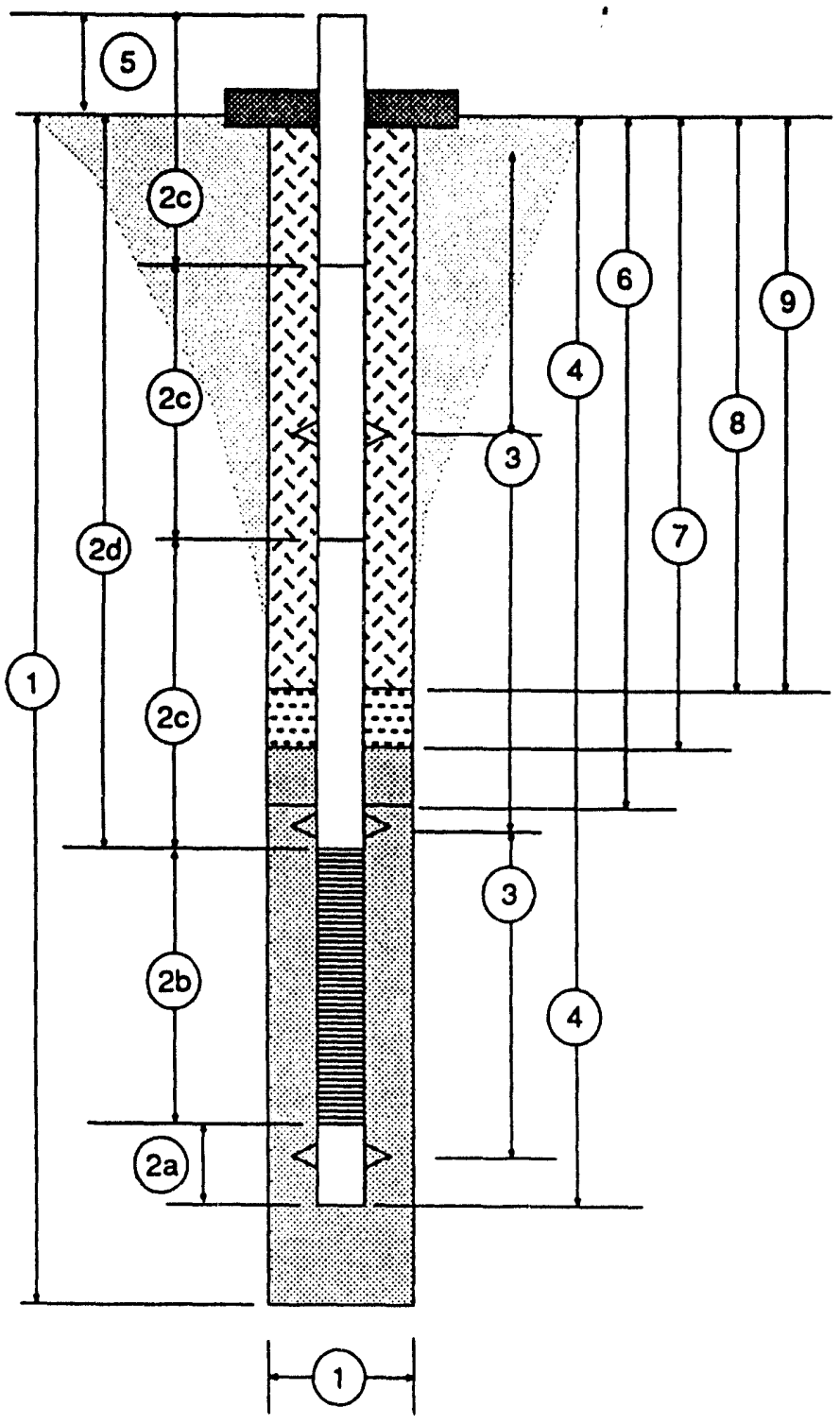

WELL NUMBER MHV - 24B SRS COORDINATES

SANITARY SEAL ELEVATION

NOTE: ALL MEASUREMENTS ARE FROM GROUND SURFACE AT START OF BORING - MEASUREMENTS TO NEAREST 0.1 FOOT.

First Grout
stege
$44.4^{\circ} / 81 / 4$ 2) Casing/Screen Tally (Measured to Nearest 0.01 Foot)
(a) Plug Length
$0.02^{\circ}$

(b) Screen Length

$4.89^{\circ}$

(c) Casing Joint Lengths (Measured in Uphole Sequence From Top of Screen) 1) $9.90^{\circ}$

2) $9.88^{\circ}$ 3) $9.89^{\circ}$ 4) 8.33

(d) Depth to Top of Screen

$38.00^{\circ}$

3) Depths to Centralizers Not reguired

4) Total Depth of Installed Well $42.9^{\circ}$

5) Casing Stick Up (Standard 2.5' A.G.S.) /. 1.6

6) Depth to Top of Filter Pack $36.0^{\circ}$

7) Depth to Top of Fine Sand Seal Not reguired

8) Depth to Top of Bentonite Seal Nut regvired

9) Thickness of Grout $3.0^{\circ}$ 
DRILLING SUBCONTRACTOR EMTC DRILLER Blake Cabit DATE OF WELL INSTALLATION $12 / 01 / 92$ TECH. O.S./CO. NAME Bill Joyce/SEC Donohve

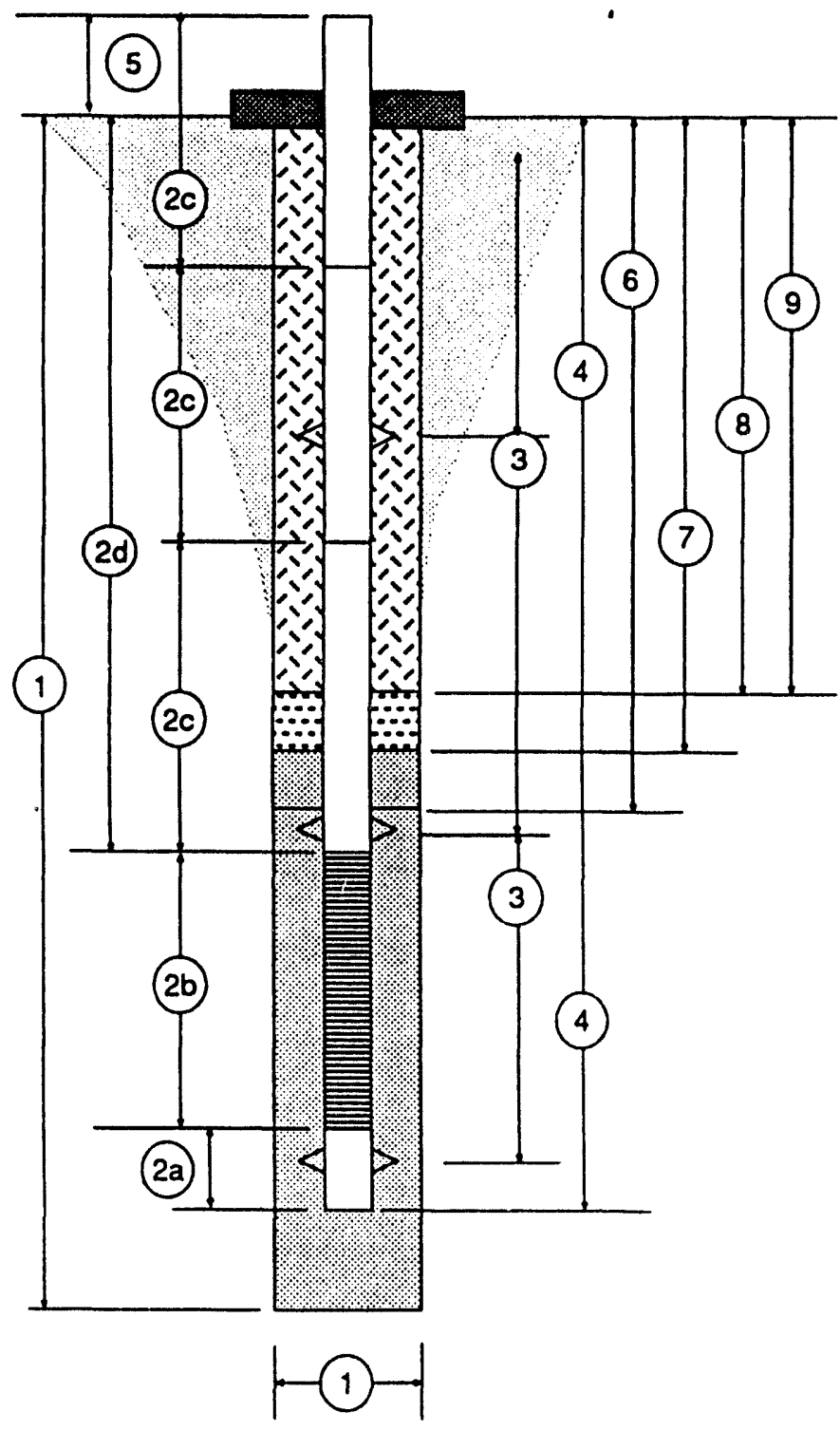

WeLL NUMBER MHV-24C SRS COORDINATES

SANITARY SEAL ELEVATION

NOTE: ALL MEASUREMENTS

ARE FROM GROUND SURFACE AT START OF BORING - MEASUREMENTS TO NEAREST 0.1 FOOT.
1) Total Drilled Depth/Hole Diameter $\begin{gathered}\text { Second Grout } \\ \text { stage } \\ 33.0^{\circ}\end{gathered}$

2) Casing/Screen Tally (Measured to Nearest 0.01 Foot)

(a)

(b) Screen Length

$4.89^{\circ}$

(c) Casing Joint Lengths (Measured in Uphole Sequence From Top of Screen) 1) $9.88^{\circ}$ 2) 9.89
3) 8.23

$28.00^{\prime}$

3) Depths to Centralizers Not reguired

4) Total Depth of Installed Well $32.9^{\circ}$

5) Casing Stick Up (Standard 2.5' A.G.S.) $1.2^{\prime}$

6) Depth to Top of Filter Pack 26.6

7) Depth to Top of Fine Sand Seal Not regvired

8) Depth to Top of Bentonite Seal Not reguired

9) Thickness of Grout $26.6^{\circ}$ 
PAge

of 2

SEC DONOHUE

CLIENT WSRC
CALCULATION SHEET

LOCATION JAS JOB NO. G. 4346 BY Bill Joyce DATE $12 / 01 / 92$ CHECKED BY DATE

From.

Cotton

Casing

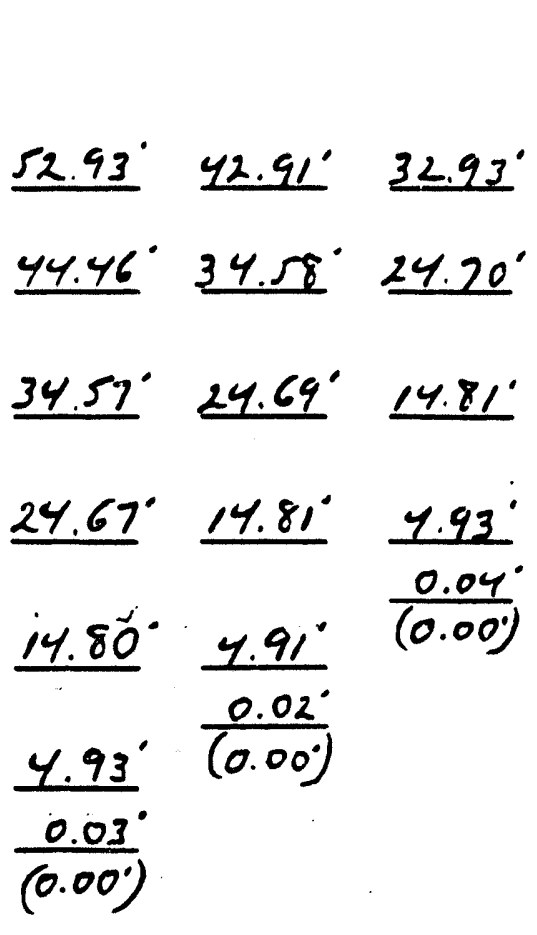

from

Ground

Surface
Gravel Pack

Based on Practical

Application in the Field of

1 ba, Coarse Sand $=1.2 \mathrm{Ht}^{3}$

bag Grot $=0.6 \mathrm{ft}^{3}$

Thus Miked $=1.3 \mathrm{ft}^{3}$
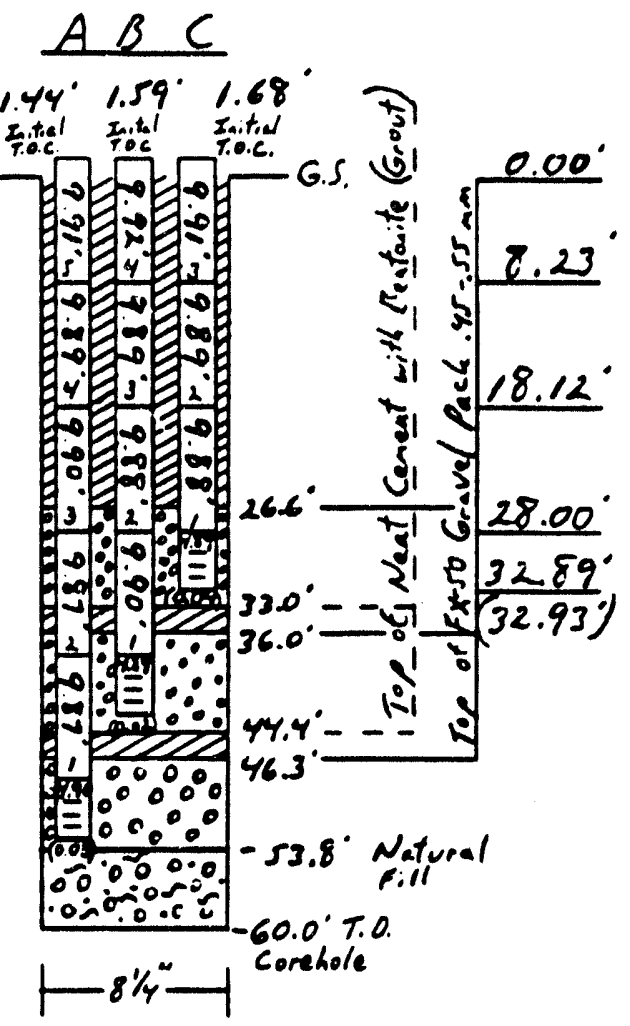

A. Theoretical
Coarse Sand - $2.2 \mathrm{bag}$
Grout - 0.6 bags

Actual

Coarse Sand - $3.3 \mathrm{bag}$ Grout -0.7 bags

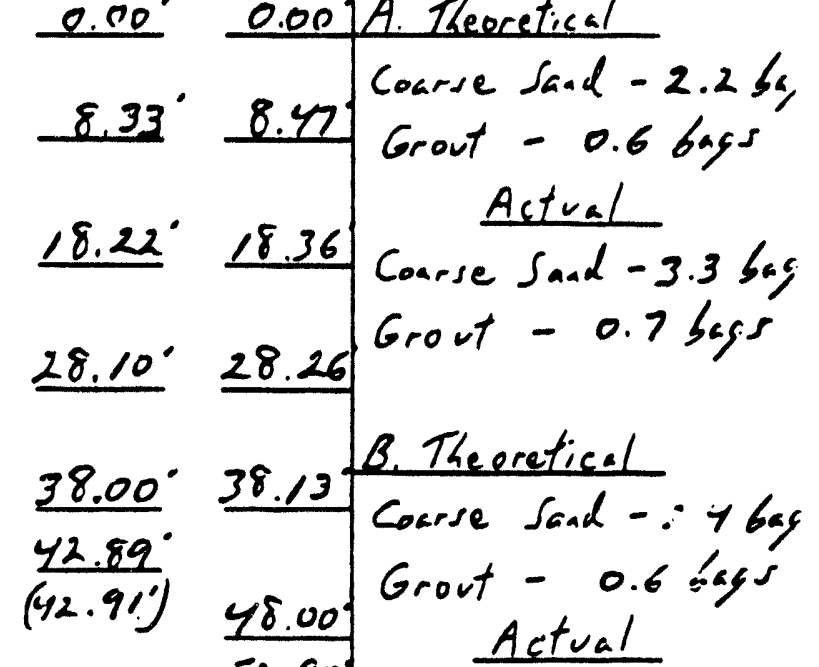

Actual
Coarse Sand - 3 bags Grout - 0.7 bags $^{\circ}$

c. Theoretical

Coarse Sand -2.3 bay

Grout - 7.2 bags

Actual

Course Sand -2 lags Grout - 6.7 bags 


$$
\text { PACE } 2 \text { O } 2
$$

SEC DONOHUE

CLIENT WSRC
CALCULATION SHEET

LOCATION

SRA

JOB NO. G4346

SUBJECT RE Heating Test - MHV - 24

Pezometers Canine Tally

CHECKED BY

DATE

Screen / Plug

Tally

A

$\frac{\text { Casing }}{\text { "PvC }}$

Schedule yo

Tri-Loc Threads

Flush

Tinco Brand

$\frac{\text { Screen }}{\text { "PVC }}$

\begin{tabular}{l|l|l|}
\hline \multirow{4}{*}{$4.90^{\circ}$} & & $0.09^{\circ}$ \\
\cline { 2 - 3 } & $\equiv$ & \\
& & \\
& & \\
& & $0.69^{\circ}$ \\
\cline { 2 - 3 } & & $0.14^{\circ}$ \\
\hline $0.03^{\circ}$ & plug & $0.03^{\circ}$ \\
\hline
\end{tabular}

slotted

0.010 in. slot

Flush

Tri-Loc Threads

Timco Brand

Sump

Not required

plug

$\beta$

\begin{tabular}{l|l|l|}
\hline & & $0.04^{\circ}$ \\
\cline { 2 - 2 } & $\equiv$ & \\
$4.89^{\circ}$ & $\equiv$ & $4.72^{\circ}$ \\
& $\equiv$ & \\
\hline & & $0.13^{\circ}$ \\
\hline $0.02^{\circ}$ & plug & $0.02^{\circ}$ \\
\hline
\end{tabular}

Schedule yo

Tri-Loc Treads

Flush

Timco Brand

s.

3) $\frac{9.91^{\circ}}{29.68^{\circ}}$

$-2800^{\circ}$ Top of Casing

$1.68^{\circ}$ Stick-yp

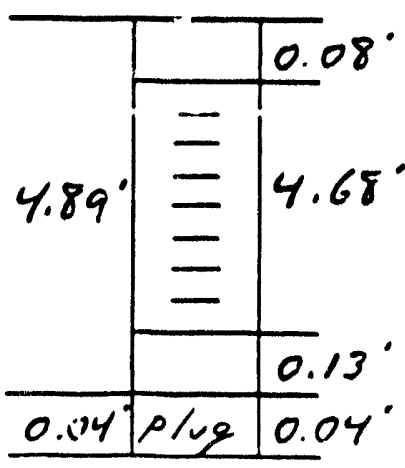




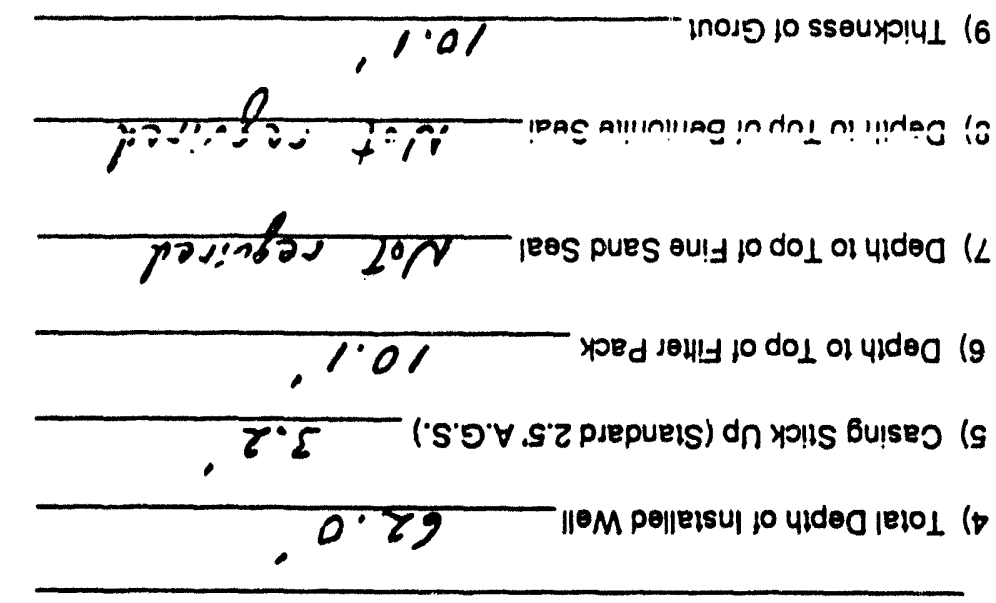

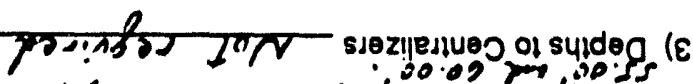

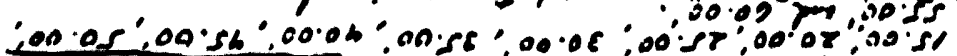

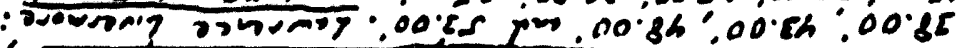

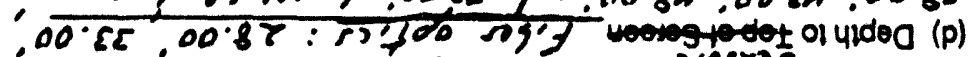

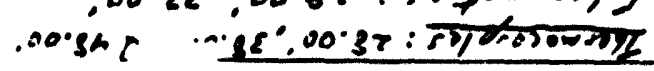

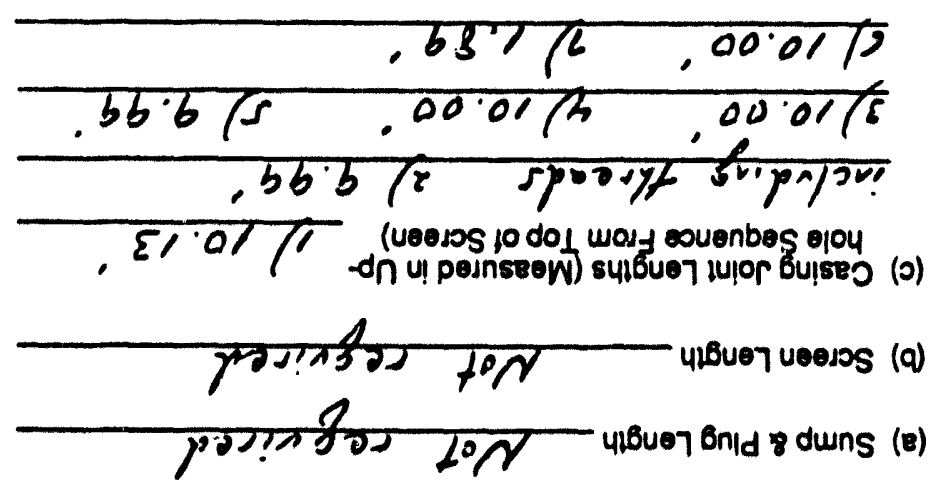

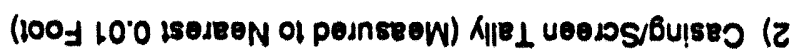

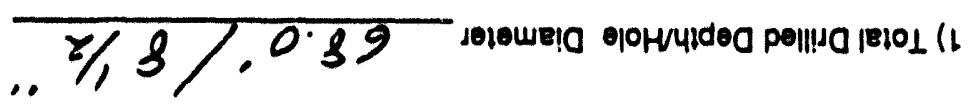

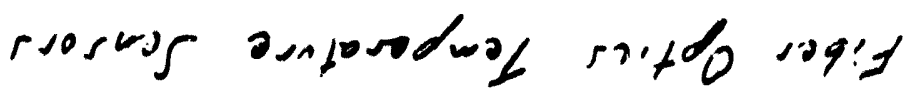

$$
\begin{aligned}
& 100 \text { - 1.0 1S } 154 \forall 3 N \text { OI }
\end{aligned}
$$

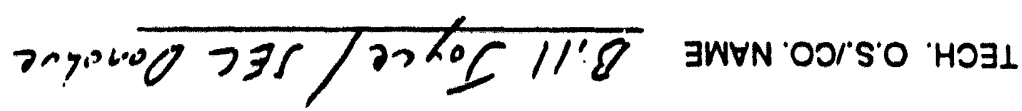

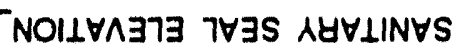
SBLVNIOYOOJ SYS $\therefore 4 T \cdot 1 / 4$ y

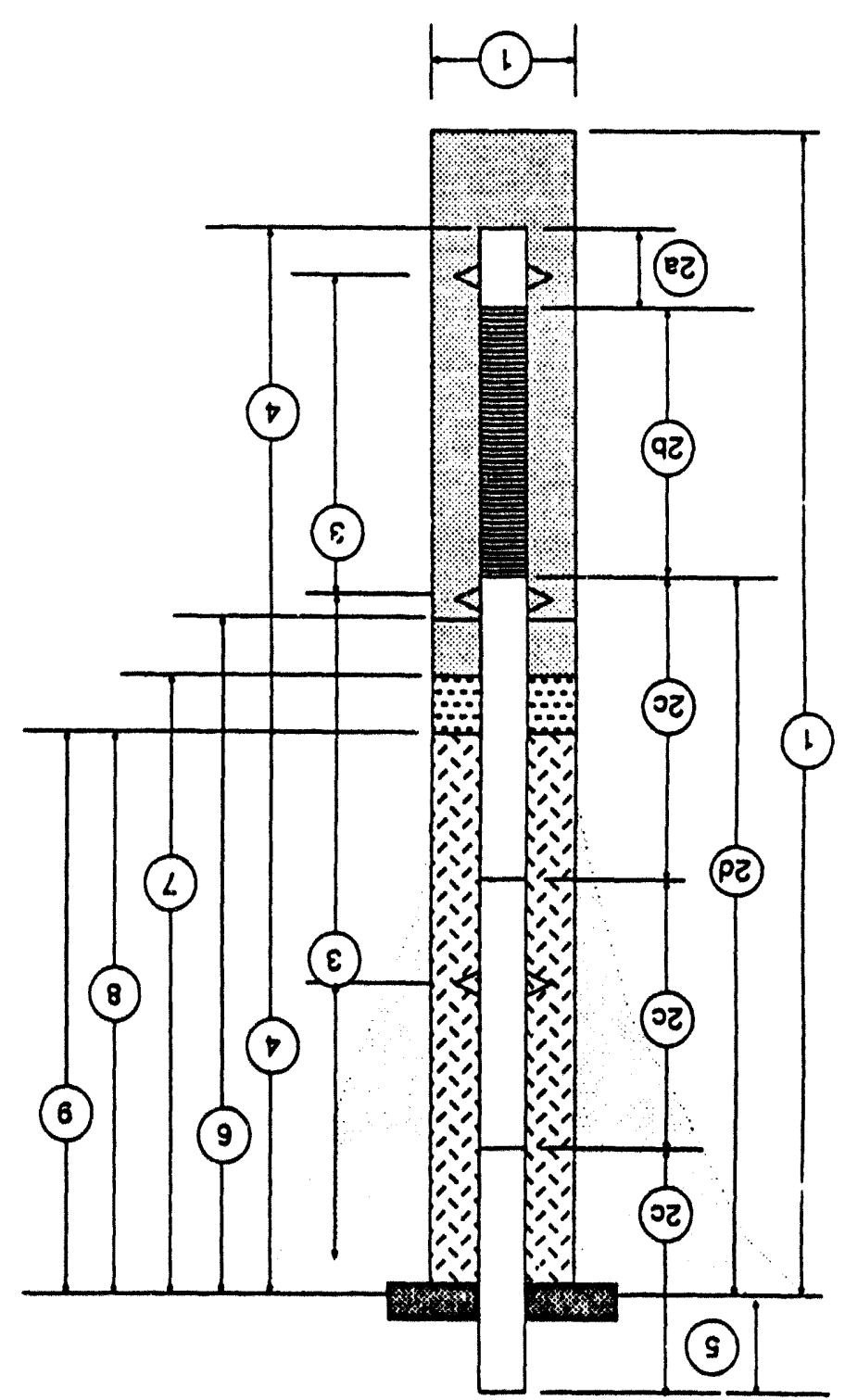

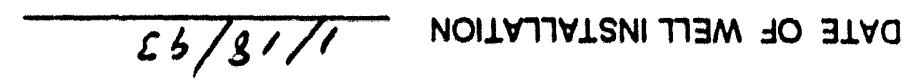
Trazy 7nats y37าוषם

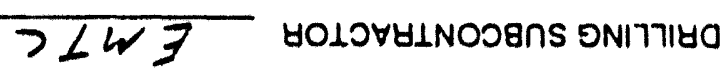


OsR 30.9

FIELD GEOLOGIC LOG

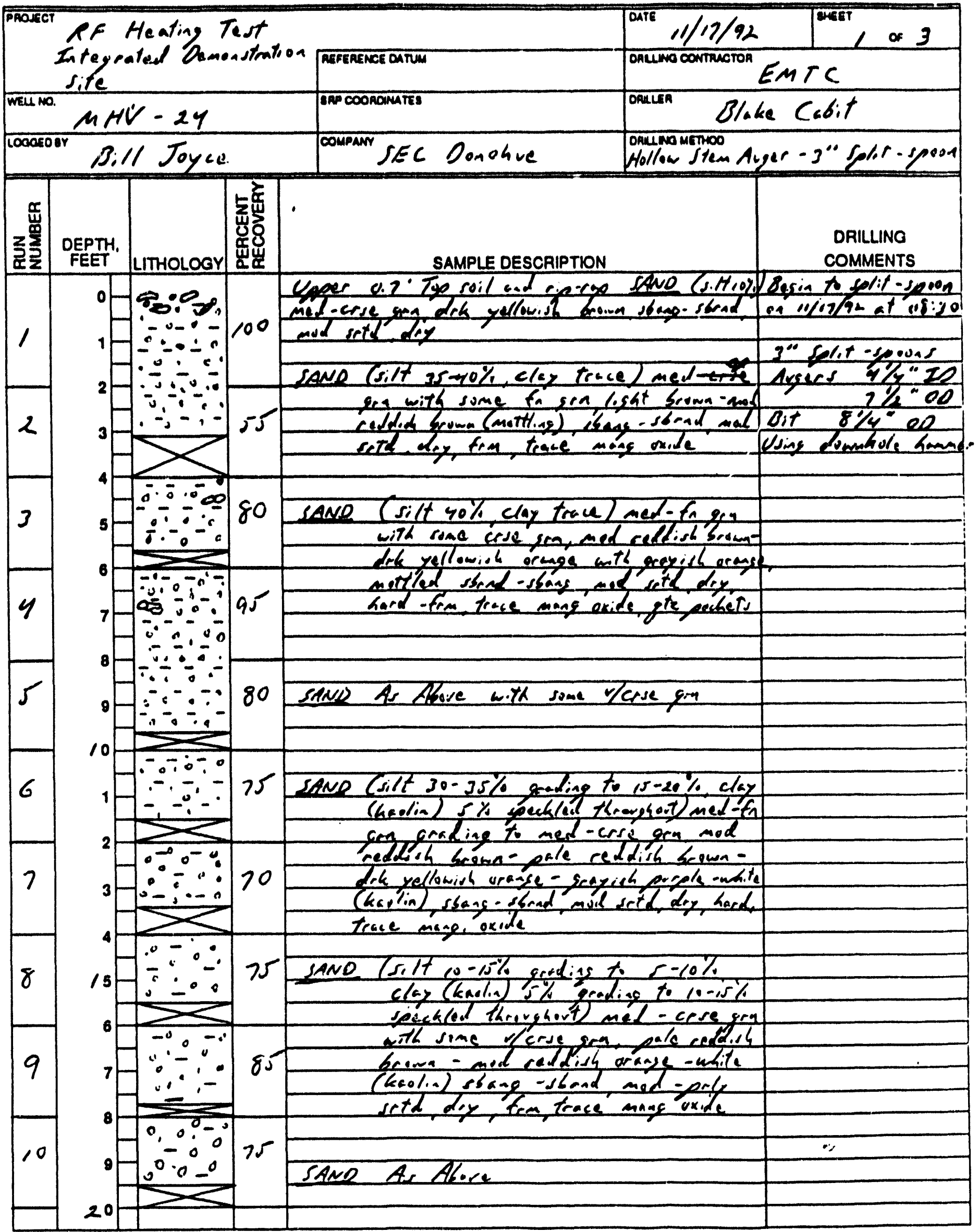


FIELD GEOLOGIC LOG

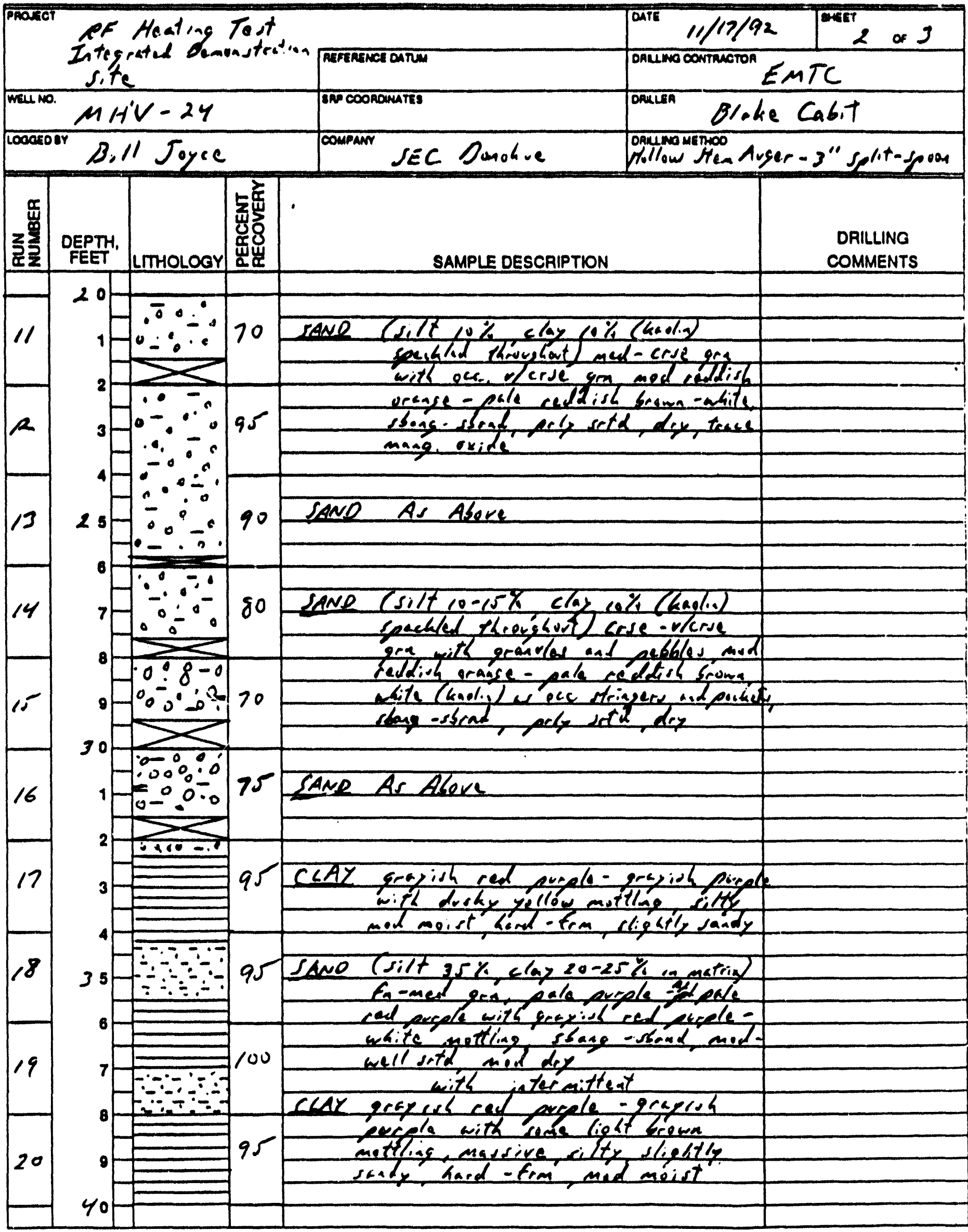




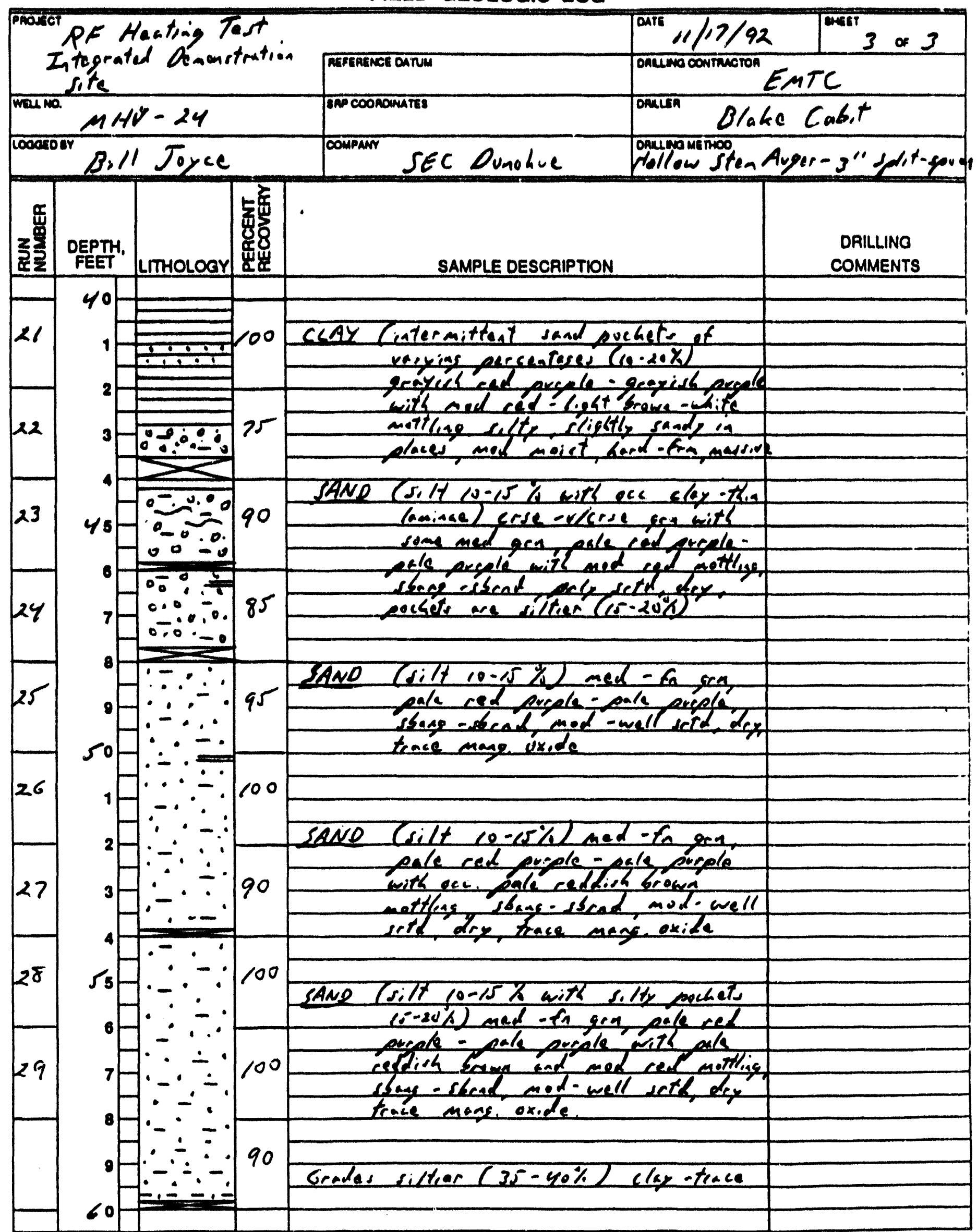


DAILLING SUBCONTRACTOR EMTE DRILLER Terry Horasery DATE OF WELL INSTALLATION $11 / 10 / 92$ tech. O.s.ico. name B.ll Tey.e/SEC Doughue

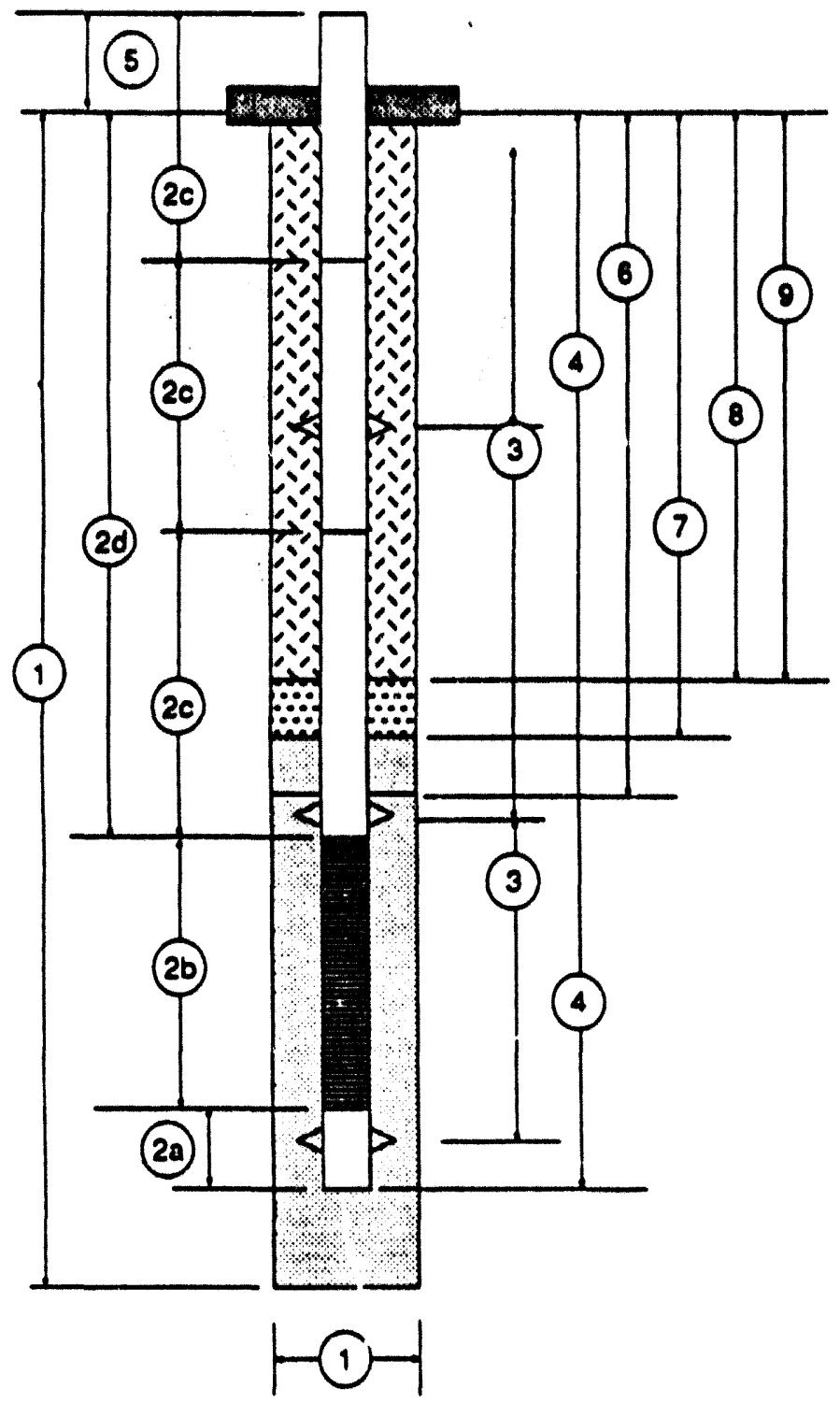

WELL NUMBER MHV. 25A SRS COORDINATES

SANITAAY SEAL ELEVATION
NOTE: ALL MEASUREMENTS ARE FROM GROUND SURFACE AT START OF BORING - MEASUREMENTS TO NEAREST 0.1 FOOT

1) Total Drillod DopthHolo Diametor $60.0^{\circ} / 81 / 4 "$

2) Casing/Scroen Tally (Mosesured to Nearest 0.01 Foot)

(a)

(B) Screen Length

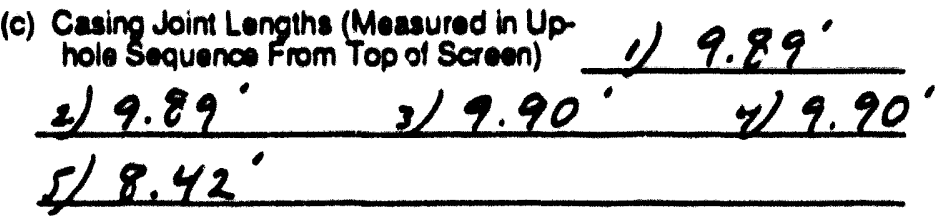

(d) Depth to Top of Screen 48.00

3) Depths to Contralizers Not reguired

4) Total Depth of Installed Woll 52.9

5) Casing Stick Up (Standard 2.5' A.G.S.)

6) Depth to Top of Finer Pack 46.6

7) Depth to Top of Fine Sand Seal Not reguired

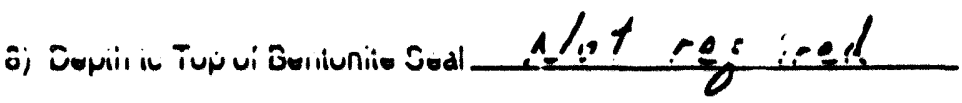

9) Thicknoss of Grout 2.4 
DRILLING SUBCONTRACTOR ENTC

DRILLER

Terey Hoensby

DATE OF WELL INSTALLATION

$11 / 11 / 92$

TECH. O.S.ICO. NAME B.ll Tuzce/rece lionohue

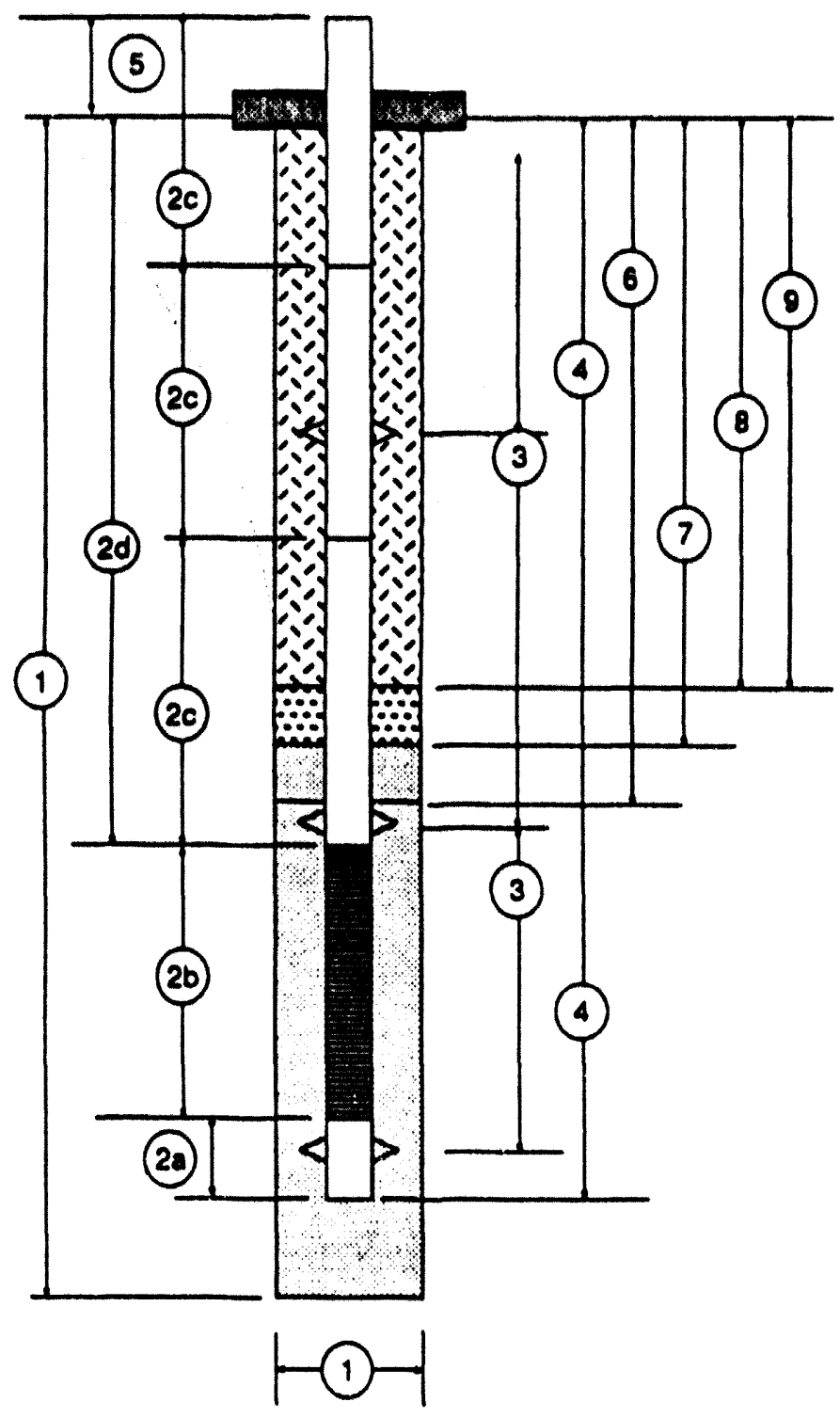

WELL NUMBER $M N V \cdot 25 B$

SRS COORDINATES

SANITARY SEAL ELEVATION

NOTE: ALL MEASUREMENTS

ARE FROM GROUND

SURFACE AT START

OF BOAING - MEASUREMENTS

TO NEAREST 0.1 FOOT.

\section{Find Growt}

1) Tolal Drilled DepthHolo Diametor $44.2, / 81 / 4 "$

2) Casing/Screon Tally (Measurnd to Noarest 0.01 Foot)

(a) Plug Length

$0.03^{\circ}$

(B) Screen Length $4.90^{\circ}$

(c) Casing Joint Lengths (Measured in Up. hole Sequence from Top of Screen), 29.89 2 $9.89^{\circ} 9.88^{-32} 4 / 8.34^{\circ}$

(d) Depth to Top of Screen

$38.00^{\circ}$

3) Depths to Contralizers Not reguired

4) Total Depth of Installed Well 42.9

5) Casing Stick Up (Standard 2.5 A.G.S.) $1.6^{\circ}$

6) Depth 10 Top of Filler Pack $36.5^{\circ}$

7) Depth 10 Top of Fine Sand Seal Not required

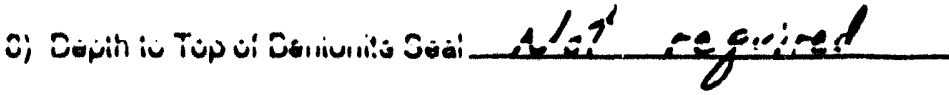

9) Thickness of Grout $2.8^{\circ}$ 
DRILLING SUBCONTRACTOR EN, E DRILLER Terey tiorn.b.e DATE OF WELL INSTALLATION $11 / 13 / 92$ TECH. O.S./CO. name B.l/ Juyced SEC Duquhue

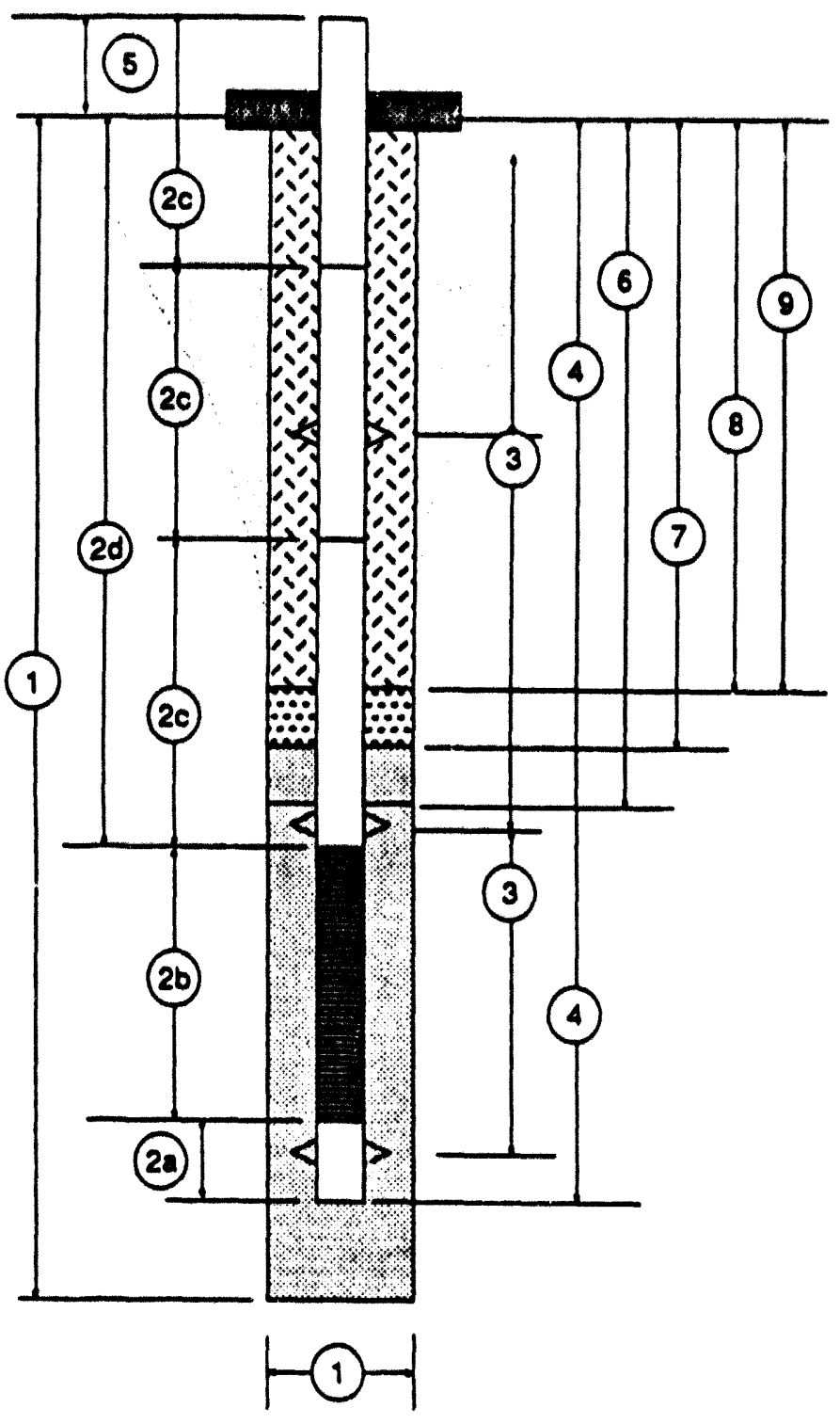

WELL NUMBER $M+1-25 L$ SRS COORDINATES

SANITARY SEAL ELEVATION

NOTE: ALL MEASUREMENTS ARE FAOM GROUND SUAFACE AT START OF BORING - MEASUREMENTS TO NEAREST 0.1 FOOT.

\section{Sesuid Geot}

1) Total Drilled DepthHolo Diamater $\begin{array}{r}199.7^{\prime} / 81 / 4 " \\ \hline\end{array}$

2) Casing/Sereen Tally (Measured to Noareat 0.01 Foot)

(a) Sumplug Lengh _ $0.02^{\prime}$

(b) Screon Longth $4.89^{\circ}$

(c) Casing Joint Longths (Mossurod in Uphole Sequence from Top of Screen) 1) $9.89^{\circ}$ 2) $9.89^{\circ}$ i $8.22^{\circ}$

(d) Depth to Top of Screen

$28.00^{\circ}$

3) Depths to Centralizers Not reguired

4) Total Depth of Installed Well $32.9^{\circ}$

5) Casing Stick Up (Siandard 2.5' A.G.S.) $1.2^{\circ}$

6) Depth to Top of Fitter Pack $26.7^{\circ}$

7) Depth to Top of Fine Sand Seal Not reguired

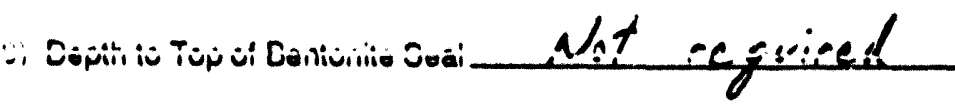

9) Thickness of Grout $26.7^{\circ}$ 
PAGE 1 OF 2

\section{SEC DONOHUE Calculation SheEt}

CLIENT WSRC LOCATION

SRS

JOB NO. G. 4346

SUBJECT RF Heating Test - MHV - 25 Piezometer Well Construction Dicgram

BY Bill Joyce DATE $11 / 13 / 92$ CHECKED BY DATE

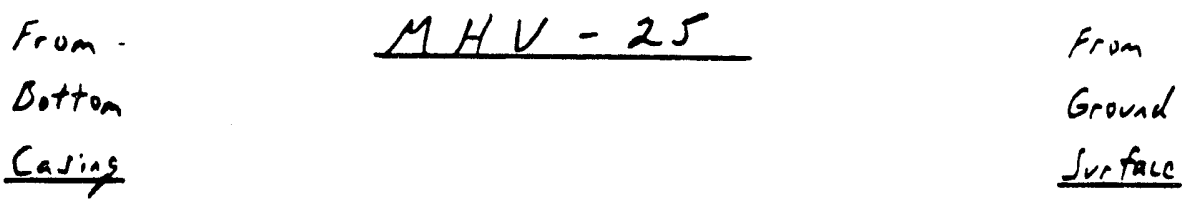

Grave/ Pack

Based on Practical

Application in the Field of

1 bag Coarse Sand $=1.2 \mathrm{ft}^{3}$

1 bag Grout $=0.6 \mathrm{ft}^{3}$

Sas Mined $=1.3 \mathrm{ft}^{3}$

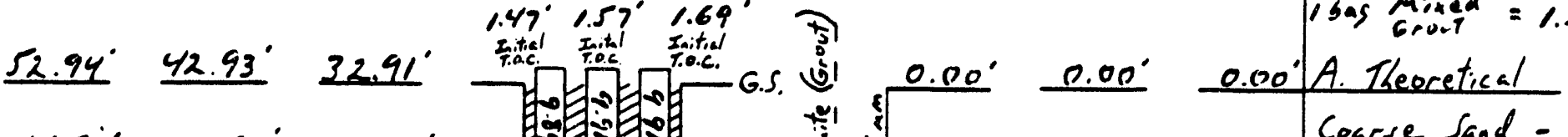

$44.52^{\circ} \quad 34.59^{\circ} \quad 24.69^{\circ}$

$34.62^{\circ} \quad 24.71^{\circ} \quad 14.80^{\circ}$

$24.72^{\circ} \quad 14.82^{\circ} \quad 4.91^{\circ}$

$14.83^{\circ}, 4.93^{\circ} \frac{0.02^{\circ}}{(0.00)^{\circ}}$

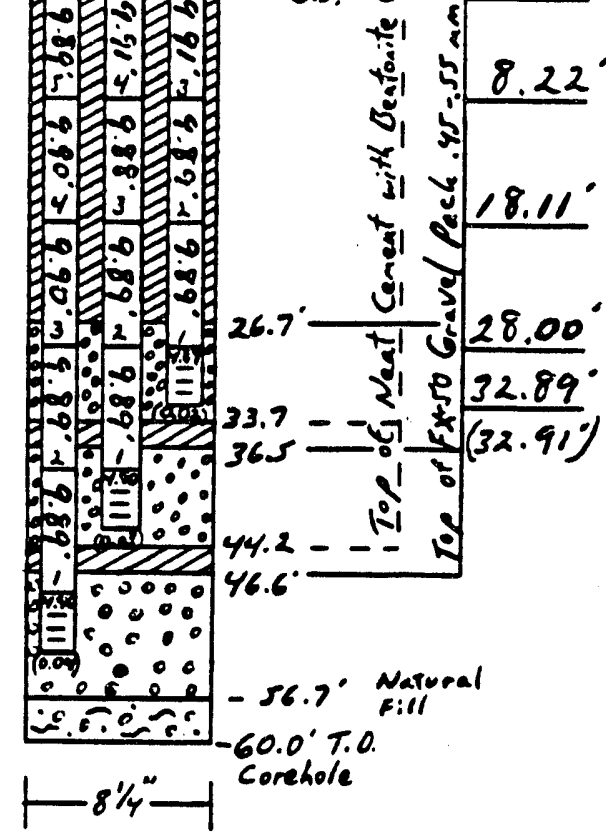

$8.34^{\circ} 8.42$

Coarse Sand - 3.1 bag

Grout - 0.6 bags

Actual

$18.22^{\circ} \quad 18.32$

Coarse Sand - 3.5 bas

Grout - 0.7 bags

$\begin{array}{ll}\frac{28.11^{\circ}}{38.00^{\circ}} & 28.22 \\ \frac{32.90^{\circ}}{\left(42.93^{\circ}\right)} & \frac{38.00^{\circ}}{} \\ \frac{52.90^{\circ}}{(52.94)}\end{array}$

B. The oretical

Course Saad - 2.4 bag.

Grout - 0.6 bags

$4.94^{\circ}\left(0.00^{\circ}\right)$

$\frac{0.04^{\circ}}{\left(0.00^{\circ}\right)}$

$$
\begin{aligned}
& \frac{\text { Actual }}{\text { Coarse Sand - } 2.5 \text { bag }} \\
& \text { Grout - } 0.7 \text { bags } \\
& \text { C Theeretical } \\
& \text { Coarse Sand - } 2.3 \text { bas } \\
& \text { Grout - } 7.2 \text { bags } \\
& \text { Actual } \\
& \text { Coapse Sand - } 1 \text { bag } \\
& \text { Grout - } 5.7 \text { bags }
\end{aligned}
$$

$+14$ 
PAge 2 OF 2

SEC DONOHUE

CLIENT WSRC
CALCULATION SHEET

LOCATION

SRA

JOB NO. G4346

SUBJECT RF Heating Test - MHV - 25

Pedometers Casing Tally

BY Bill Joyce DATE $11 / 13 / 92$ CHECKED BY

DATE

$\frac{\text { Casing }}{\text { Tally }} \frac{\text { Screen }}{\frac{A}{9.89^{\circ}}} \cdot \frac{A}{48.0^{\circ}-53.0}$

2) $9.89^{\circ}$

3) $9.90^{\circ}$

4) $9.90^{\circ}$

5) $\frac{9.89^{\circ}}{49.47^{\circ}}$

$-48.00^{\circ}$ Top of Casing

$1.47^{\circ}$ stick-up

$\frac{B}{9.89^{\circ}} \cdot \frac{B}{38.0^{\circ}-43.0^{\prime}}$

2) $9.89^{\circ}$

3) $9.88^{\circ}$

4) $\frac{9.91^{\circ}}{39.59^{\circ}}$

$\frac{-38.00^{\circ}}{159^{\circ}}$ Top of Cawing

Materials

List $(A, B C)$

Screen / plug

$\frac{T_{a} / l y}{A}$

$\frac{\text { Casing }}{1 " \text { Pvc }}$

Schedule yo

Tri-Loc Threads

Flush

Timco Brand

$\frac{\text { Screen }}{1 \text { PVC }}$

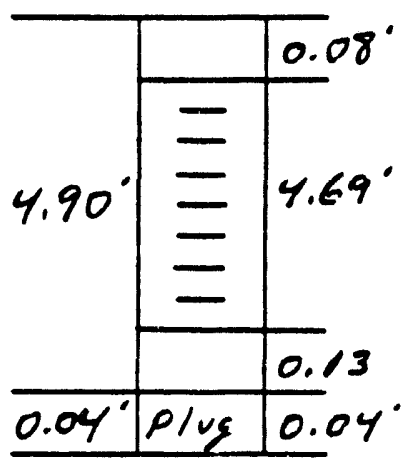

slotted

$0.010 \mathrm{in}$. slot

Flush

Tri-ioc Threads

Since Brand

Sump

Not required

plug

$\beta$

\begin{tabular}{l|l|l|}
\hline & & $0.07^{\circ}$ \\
\cline { 2 - 3 } & $=$ & \\
$4.90^{\circ}$ & $\equiv$ & $4.69^{\circ}$ \\
& $\equiv$ & \\
& & \\
\cline { 2 - 3 } & & $0.14^{\circ}$ \\
\hline $0.03^{\circ}$ & plug & $0.03^{\circ}$ \\
\hline
\end{tabular}

Schedule yo

Tri-Loc Threads

Flush

Timco Brand

3) $\frac{9.91^{\circ}}{29.69^{\circ}}$

- 28.00 $0^{\circ}$ Top of Casing

$1.69^{\circ}$ St.ck-yp

c

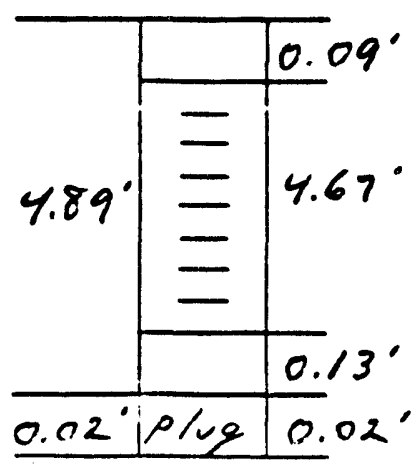




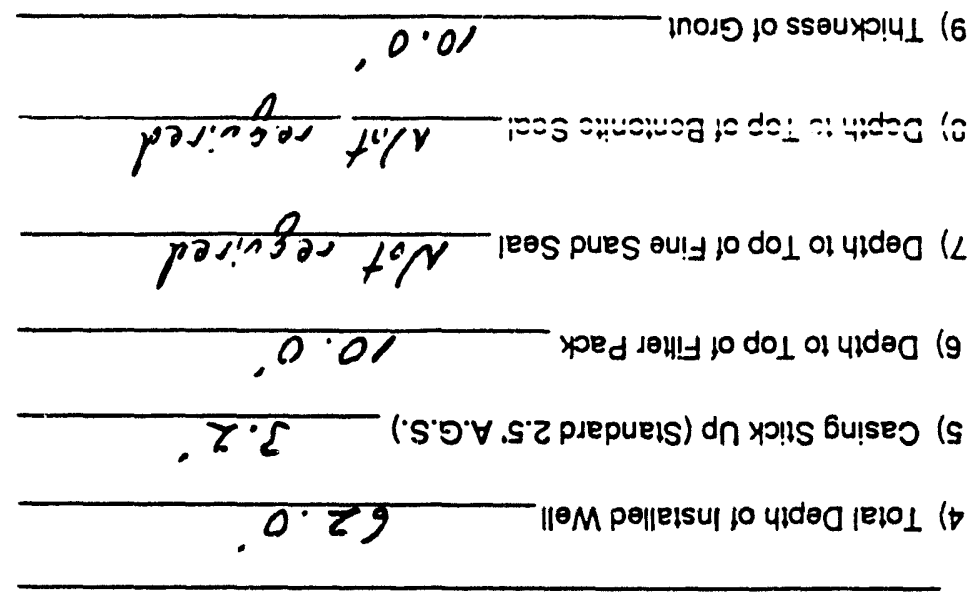

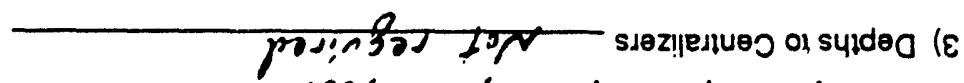
$\because 00.09$ 7:0.00.55:.000.05:.0054:00.04:00.58:00.06

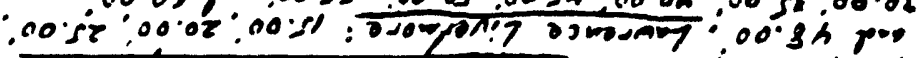

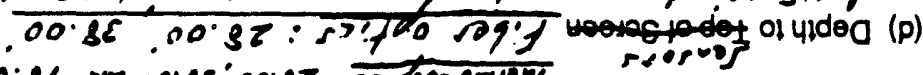

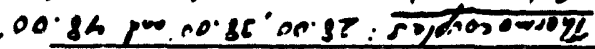

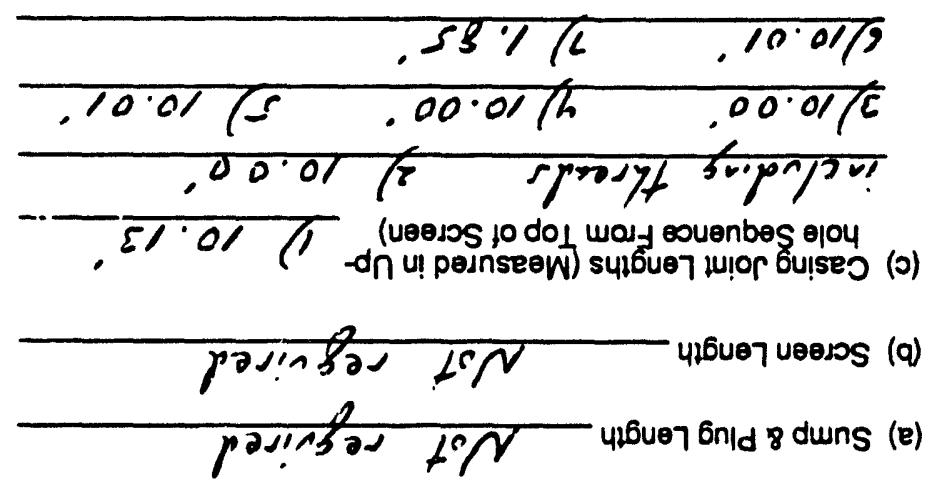

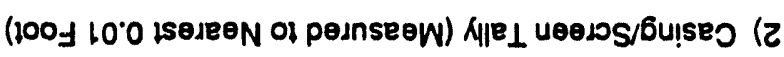

$$
\begin{aligned}
& \text {. }
\end{aligned}
$$

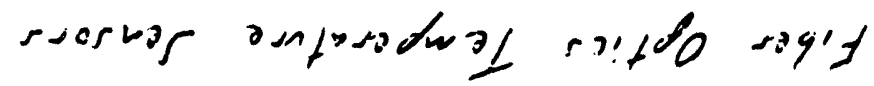

$$
\begin{aligned}
& 100 \exists 1.0 \text { LS } 18 \forall \exists N \text { OL }
\end{aligned}
$$

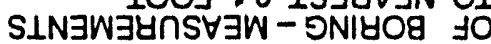

$$
\begin{aligned}
& \perp \forall \forall \perp S \perp \forall \quad \exists O \forall J \forall \cap S \\
& \text { aNnOy WOH } \exists \forall \forall
\end{aligned}
$$

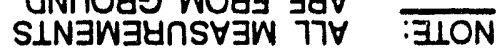
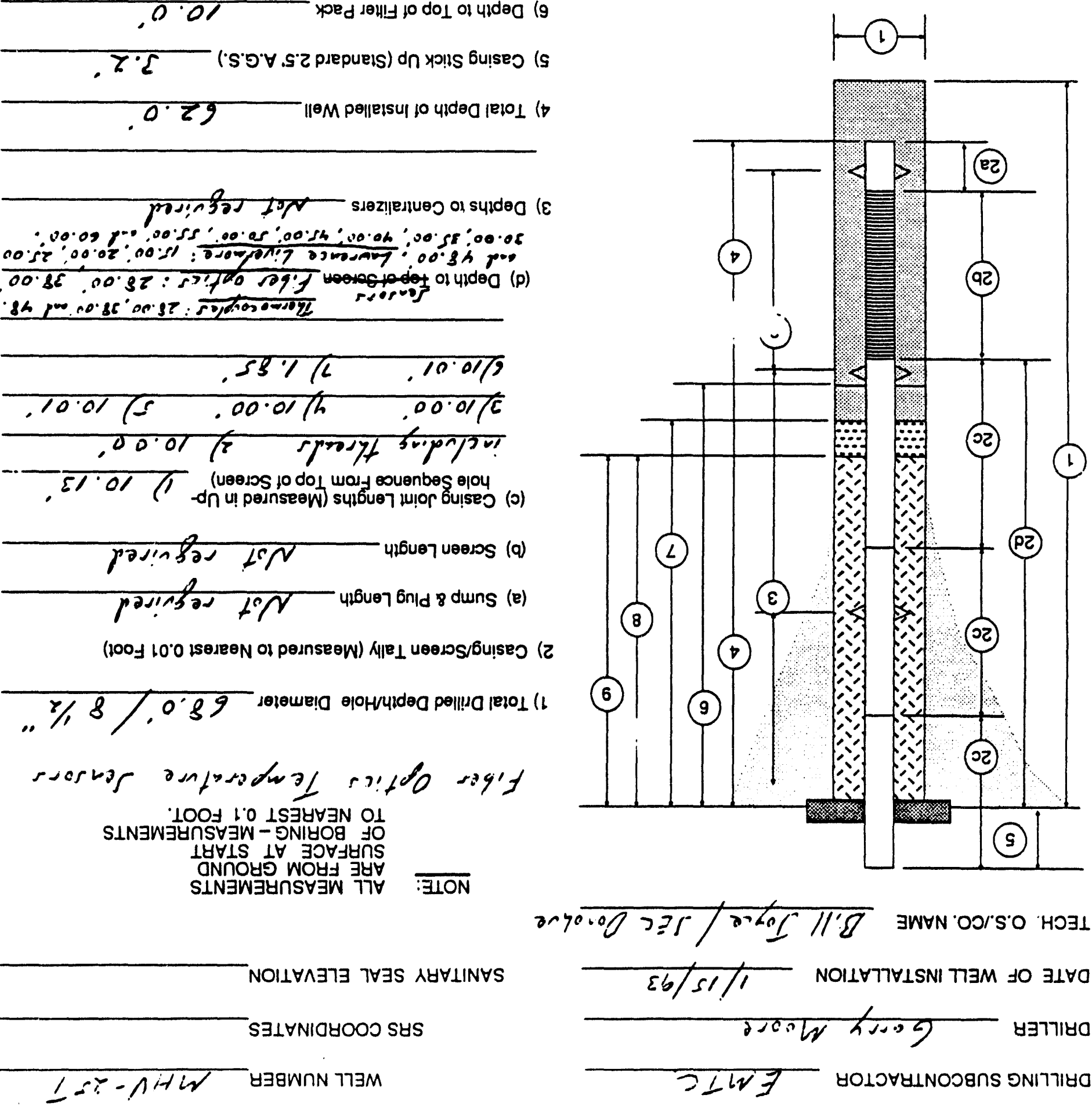

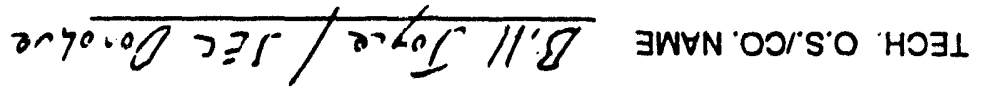

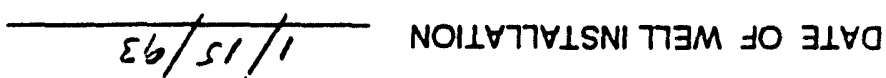

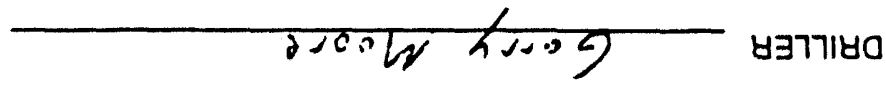

$$
\begin{aligned}
& \text { วเปข }
\end{aligned}
$$




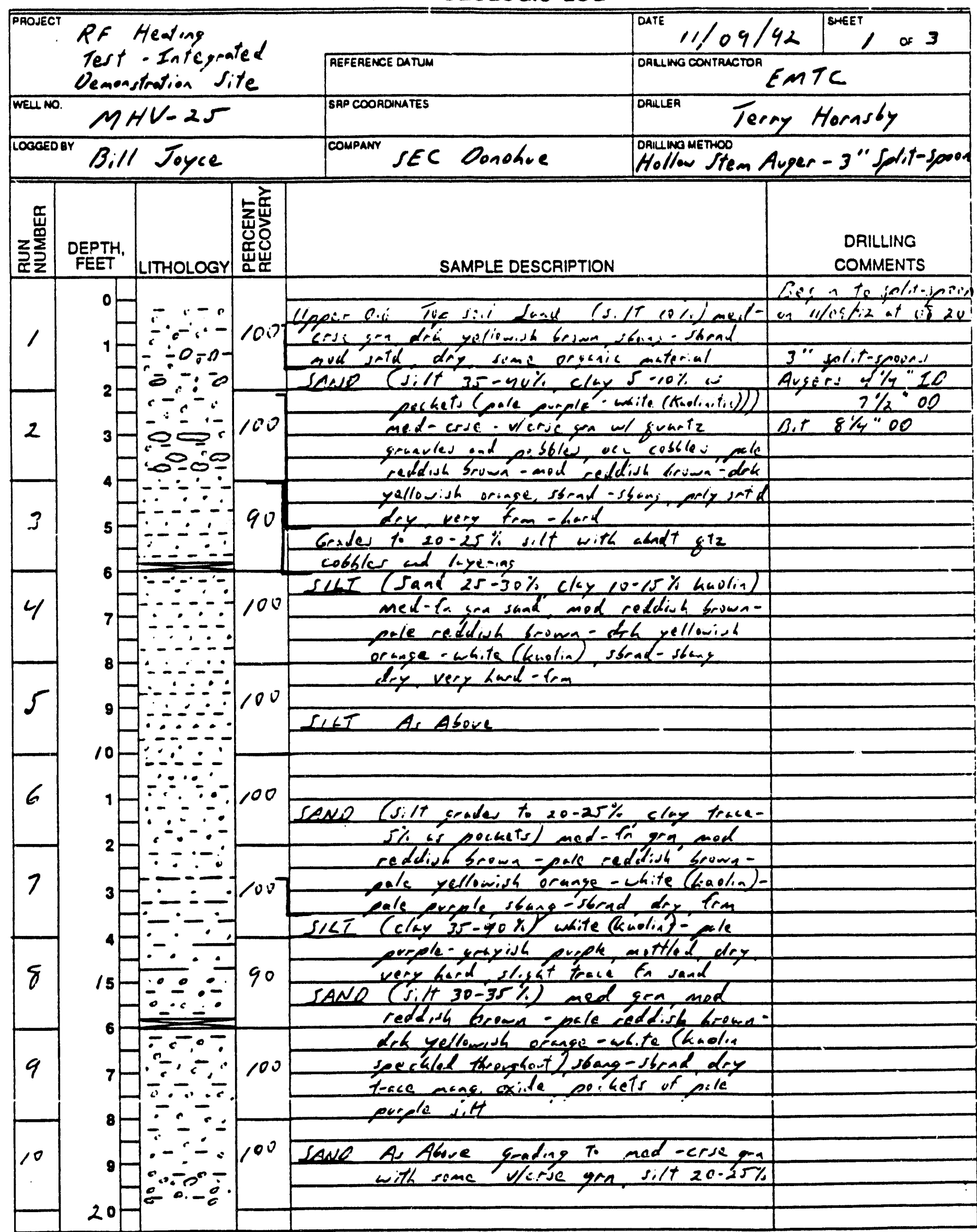




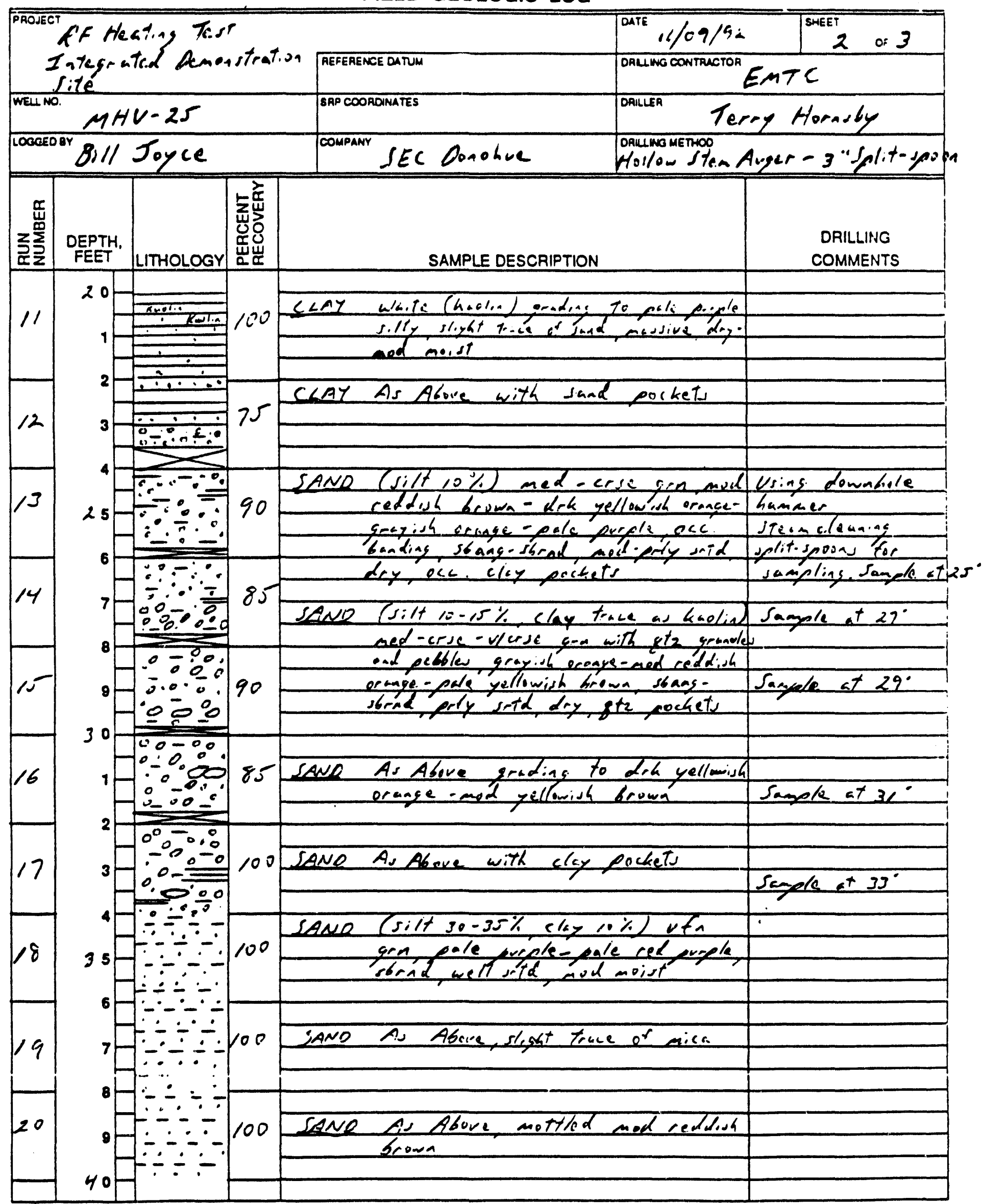




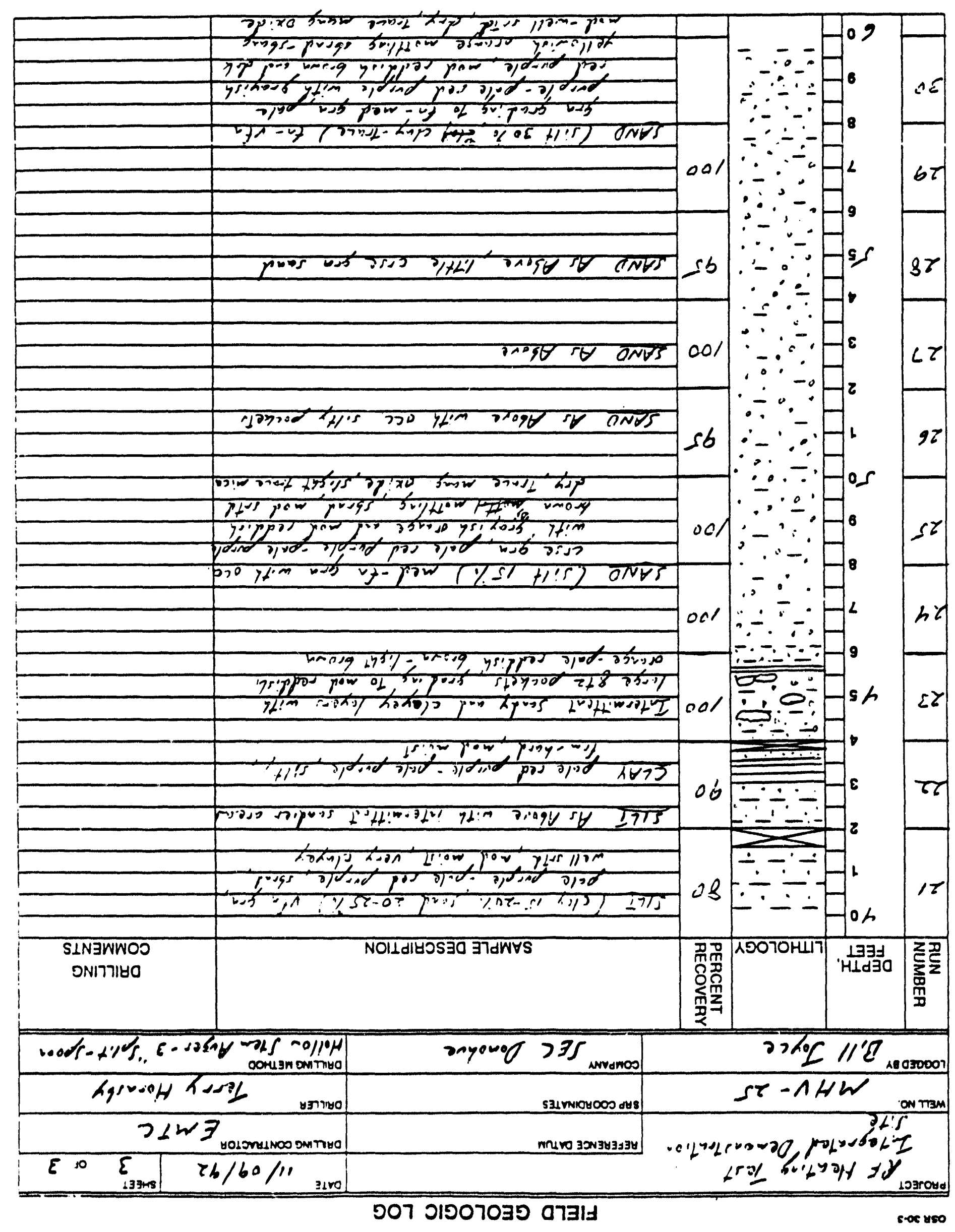


DRILLING SUBCONTRACTOR

EMTC

DRILLER

$$
\text { Tirey hionsicy }
$$

DATE OF WELL INSTALLATION

$11 / 11 / 92$

TECH. O.S.ICO. NAME Bili, Toyie/SEC fuavhue

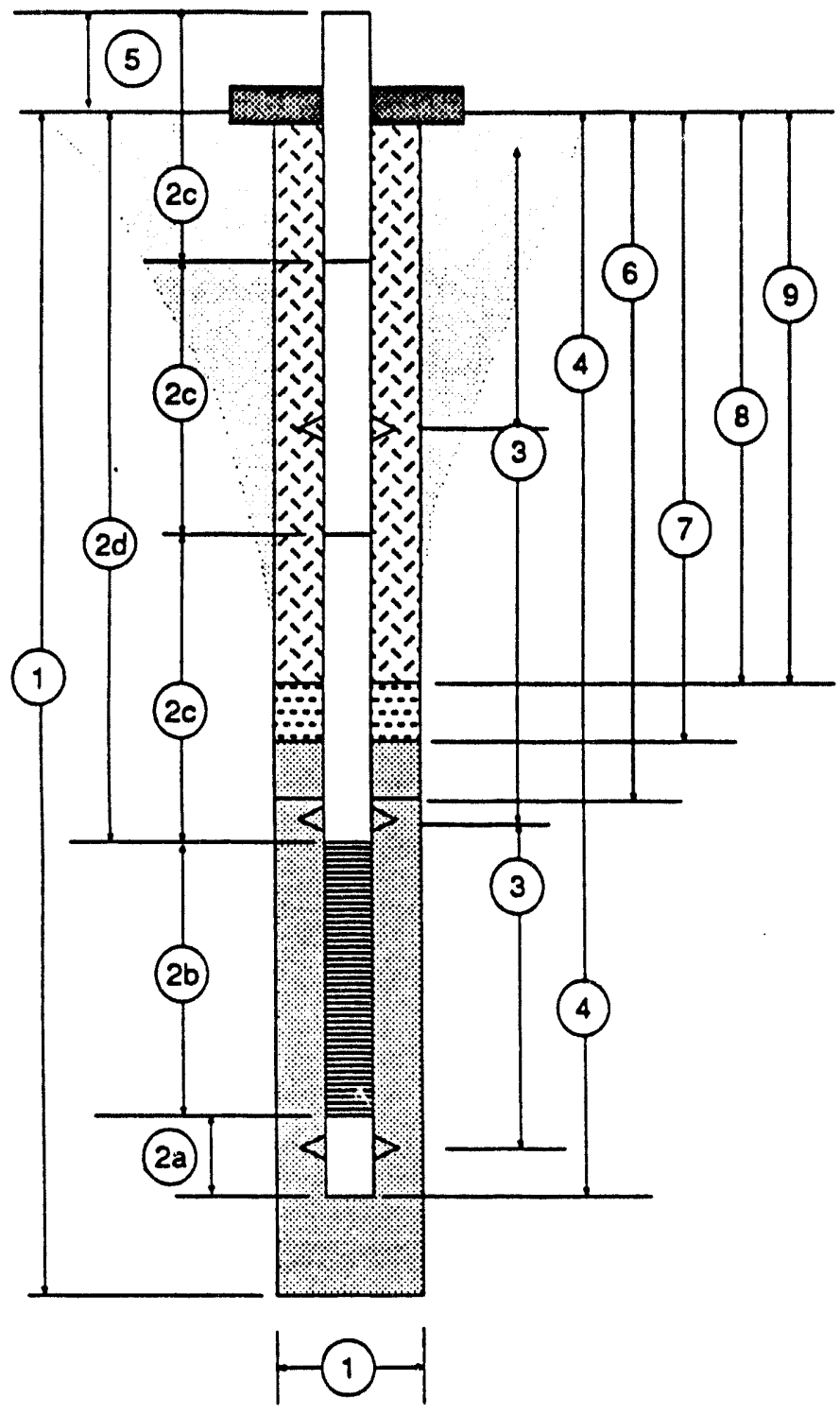

WELL NUMBEF__. $11 \mu V^{\prime} \cdot 26 A$ SRS COORDINATES

SANITARY SEAL ELEVATION

NOTE: ALL MEASUREMENTS

ARE FROM GROUND

SURFACE AT START

OF BORING - MEASUREMENTS

TO NEAREST 0.1 FOOT.

1) Total Drilled Depth/Hole Diameter $60.0^{\circ} / 81 / 4$

2) Casing/Screen Tally (Measured to Nearest 0.01 Foot)

(a) Plug Length 0.02

(b) Screen Length 4.91

(c) Casing Joint Lengths (Measured in Uphole Sequence From Top of Screen) $1 / 9.90^{\circ}$ 2) $9.93^{\prime}$ 3) $9.89^{\circ}$ 4) $9.92^{\circ}$ 5) $8.36^{\circ}$

(d) Depth to Top of Screen $48.00^{\circ}$

3) Depths to Centralizers Not regrired

4) Total Depth of Installed Well $52.9^{\prime}$

5) Casing Stick Up (Standard 2.5 A.G.S.) $1.6^{\circ}$

6) Depth to Top of Filter Pack 46.6

7) Depth to Top of Fine Sand Seal_Nct re evired

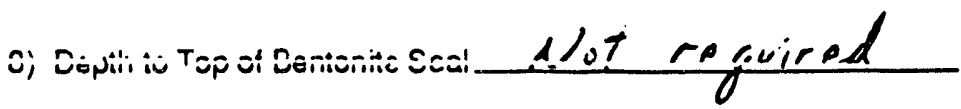

9) Thickness of Grout $2.5^{\circ}$ 

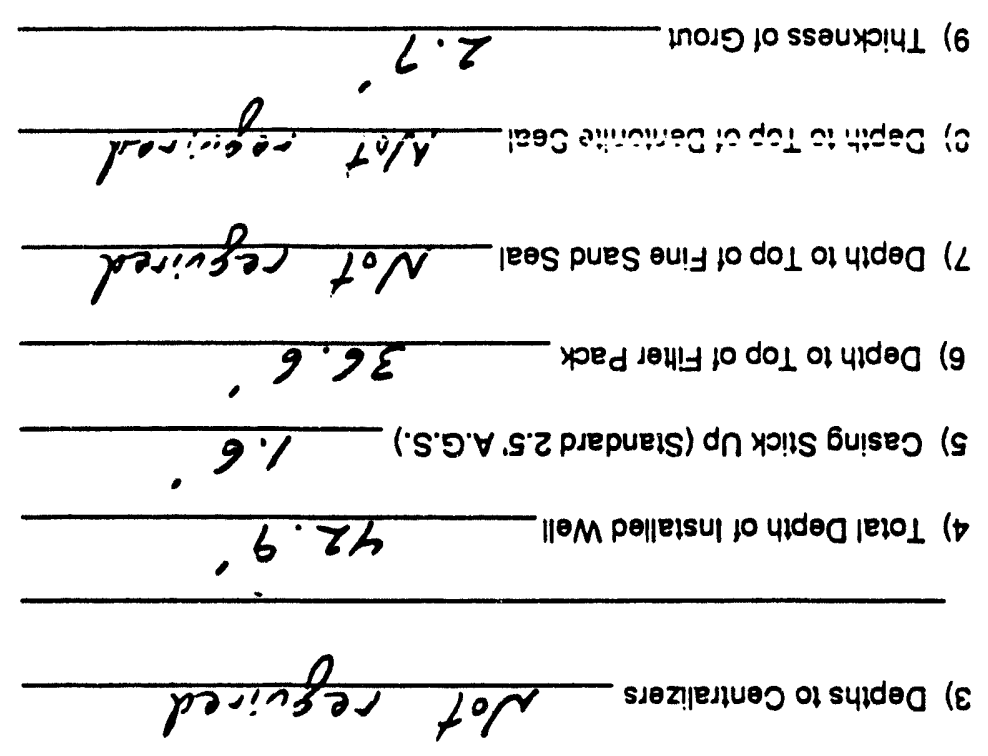

$$
.00 . \mathrm{sE} \text { veers } 10 \mathrm{do1} 01 \text { 41de0 (p) }
$$

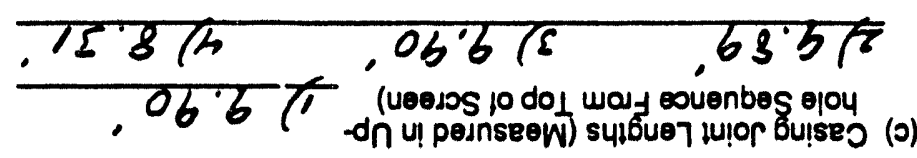

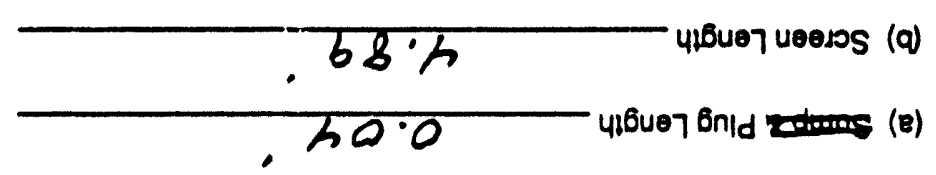

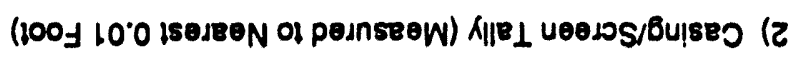

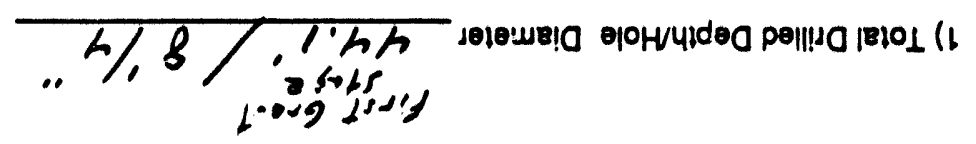

. 10091.0 1S $15 \forall \forall \exists N$ OL

SLNヨWJURS $\perp \forall \forall \perp S \perp \forall \exists O \forall J \forall \cap S$ aNnOUS WOHJ $\exists \forall \forall$

SIN

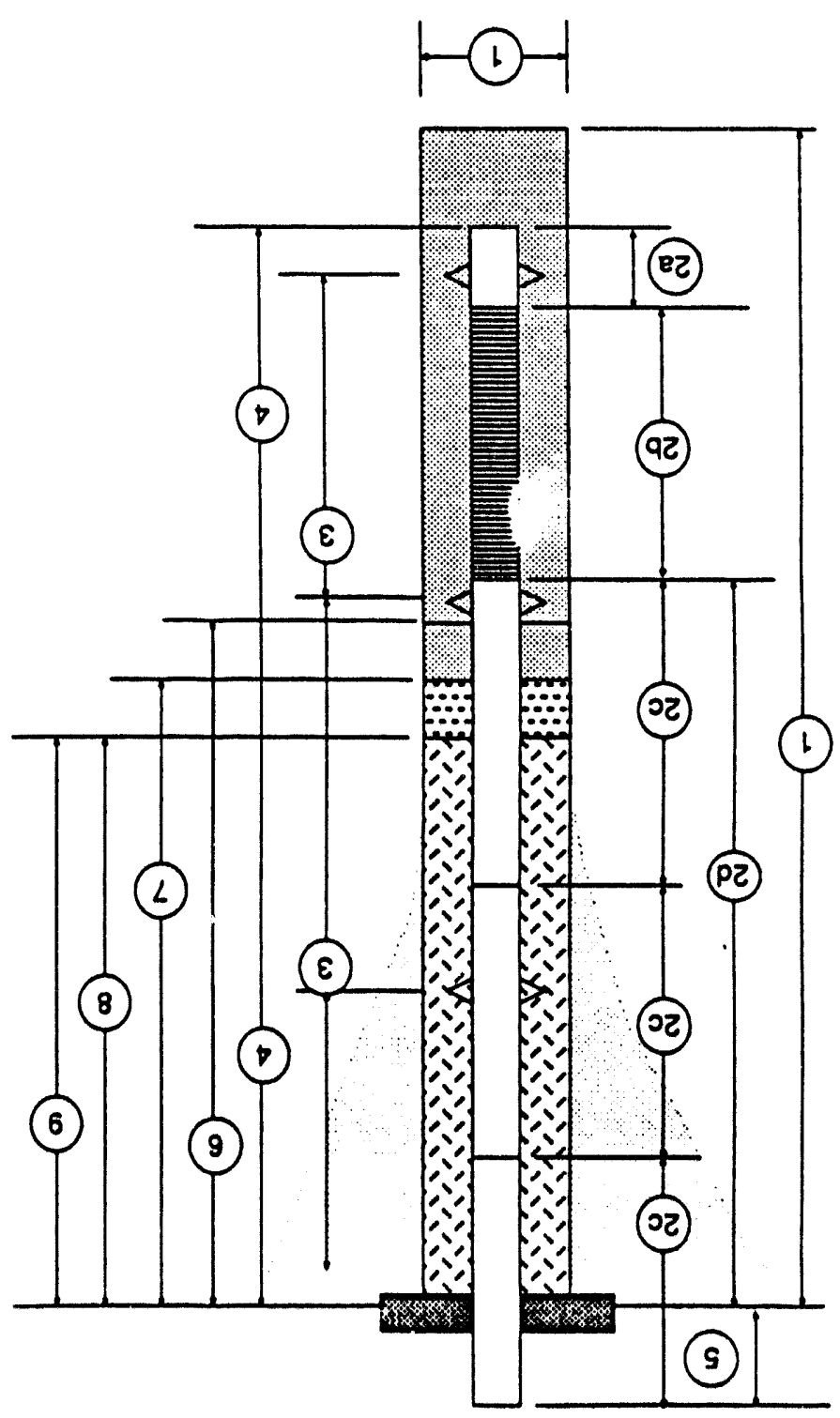

$\rightarrow$ yovoll $332 /$ कat.
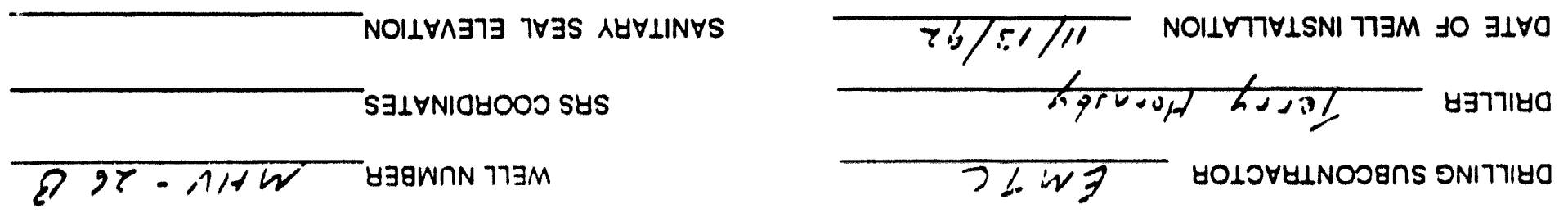
DRILLING SUBCONTRACTOR EMTC DRILLER Slake Cab,t DATE OF WELL INSTALLATION $11 / 16 /$ ic TeCh. O.s.ico.name Bill Jugle/SEC Donohue

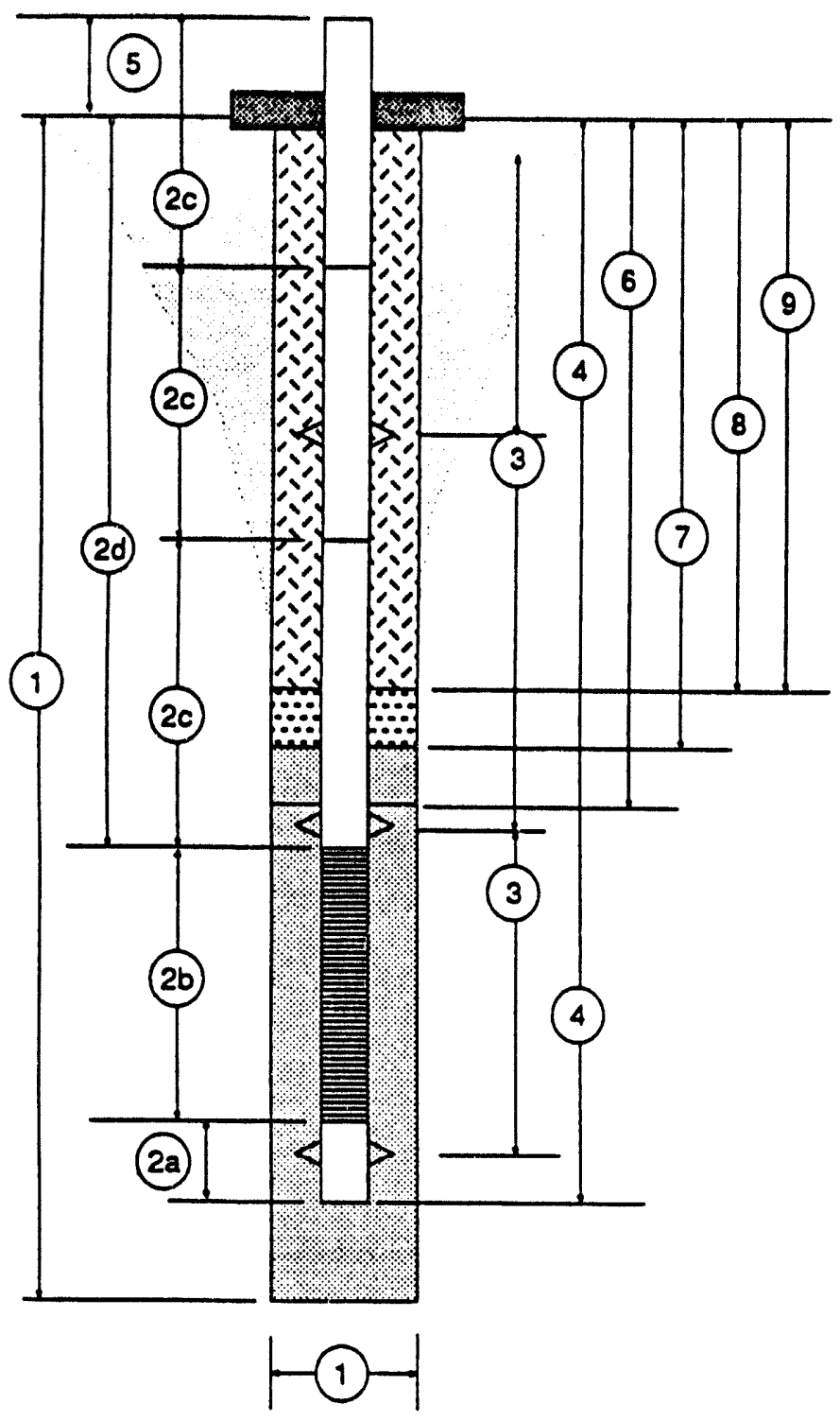

WELL NUMBER MHV.26C SRS COORDINATES

SANITARY SEAL ELEVATION 
PAQE 1 O 2

\section{SEC DONOHUE}

CALCULATION SHEET

CLIENT

WSRC LOCATION SRS JOB NO. G.4346 SUBUECT RF Heating Test - MHV - 26 Piezometer Well Construction Dicgeam BY" Bill Joyce DATE $11 / 16 /: 2$ CHECKED BY DATE

From

Cottion

Casing
$M H V-26$
Frum

Grovad

Srefrace
$\frac{A B C}{1.55^{\circ} 1.59^{\circ} 1.70^{\circ}}$

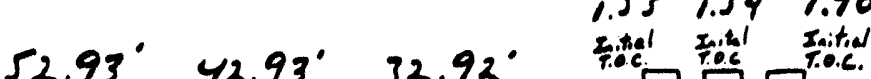

$32.93^{\circ} \quad 12.93^{\circ} 32.92^{\circ}$
$44.52^{\circ} 34.62^{\circ} 24.72^{\circ}$
$34.65^{\circ} 24.72^{\circ} 14.83^{\circ}$

$24.76^{\circ} 14.83^{\circ} 4.92^{\circ}$

$14.83^{\circ} 4.93^{\circ} \frac{0.02}{\left(0.00^{\circ}\right)}$

$4.93^{\circ} \cdot \frac{0.04}{(0.00)}$

$\frac{0.02^{\circ}}{\left(0.00^{\circ}\right)}$

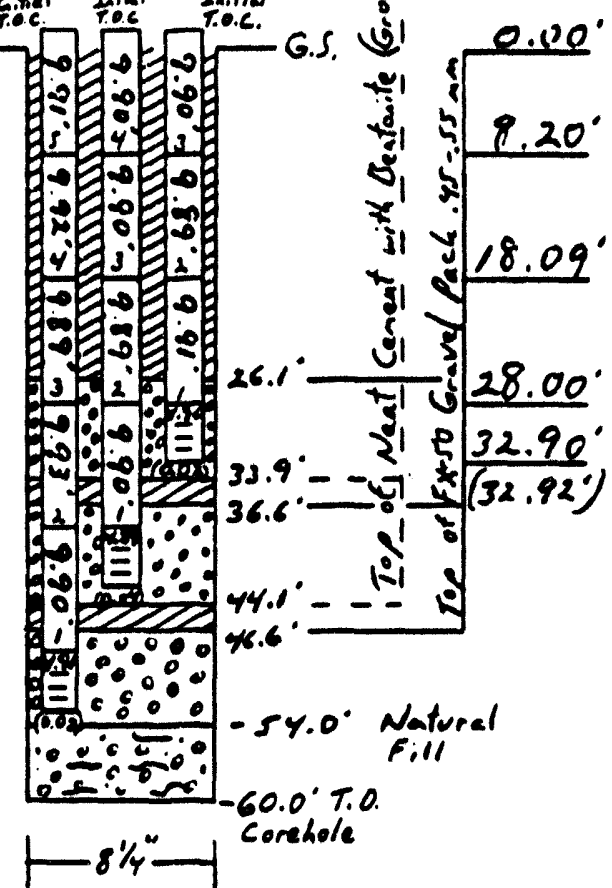

$+1+1$
Gravel Pack

Bused on Practical

Application in the Field of 1 bag Coarse Sards $1.2 \mathrm{ft}^{\circ}$ $\checkmark$ bag Grout $=0.6 \mathrm{ft}^{3}$ l bas Mixed $=1.3 \mathrm{ft}^{3}$ $0.00^{\circ} 0.00^{\circ}$ A. Theocetical Coarse Sand - 2.3 ba, Grout - 0.6 bagst Coarse Sand - 3 bay Grout - 0.7 bagd $28.10^{\prime} 28.17$

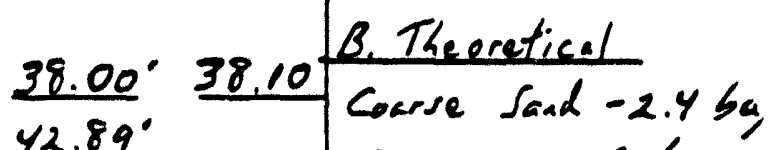
$\frac{42.89^{\circ}}{\left(42.93^{\circ}\right)}$ Grout - 0.6 bags' (42.93\% 18.00 Actual $\frac{52.91}{(52.93)}$ Coarse Sand - 2 bag. Grout - 0.7 bass cheocetical Coarse Sand-2.3 6c Grout - 1.2 bags Actual Coarse Saded - 2.3 bas Grout - 5.7 bags 


$$
\text { PAGE } 2 \text { Of } 2
$$

SEC DONAHUE

CLIENT WSRC
CALCULATION SHEET

LOCATION

SET

JOB NO. G4346

subJect RE Heating Test - MHV - 26 feezometers Casing Tally

BY B. ll Joyce DATE $11 / 16 / 92$ CHECKED BY

DATE

Screen / plug

Tally

A

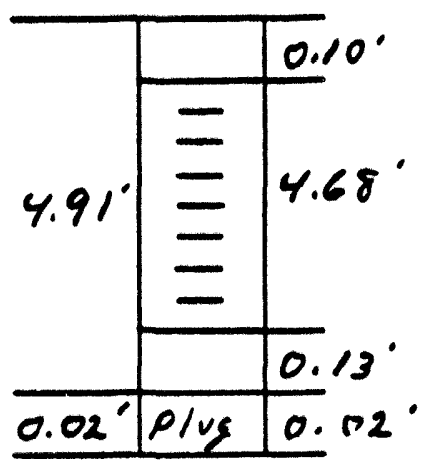

$\frac{\text { Screen }}{\text { "PVC }}$

slotted

$0.010 \mathrm{in}$. slot

Flush

…Loc Threads

Times Brand

Sump

Not required

plug

B

\begin{tabular}{l|l|l|}
\hline & & $0.07^{\circ}$ \\
\cline { 2 - 3 } $4.89^{\circ}$ & $\equiv$ & \\
& & $4.69^{\circ}$ \\
& $=$ & \\
\cline { 2 - 3 } & & 0.13 \\
\hline 0.04 & plug & 0.04 \\
\hline
\end{tabular}

Schedule yo

Tri-Loc Treads

Flush

Timco Brand c

\begin{tabular}{l|l|l|}
\hline & & $0.09^{\circ}$ \\
\cline { 2 - 2 } $4.90^{\circ}$ & $\equiv$ & $4.68^{\circ}$ \\
& $\equiv$ & \\
& $=$ & \\
\cline { 2 - 3 } & $0.13^{\circ}$ \\
\hline $0.022^{\circ}$ & plug & $0.02^{\circ}$ \\
\hline
\end{tabular}


DRILLING SUBCONTRACTOR EMTC DRILLER Grany Aquire / live fiese DATE OF WELL INSTALLATION $1 / 22 / 93$ TECH. O.S.ICO. name B.ll Toy.e/SEC Dimohue

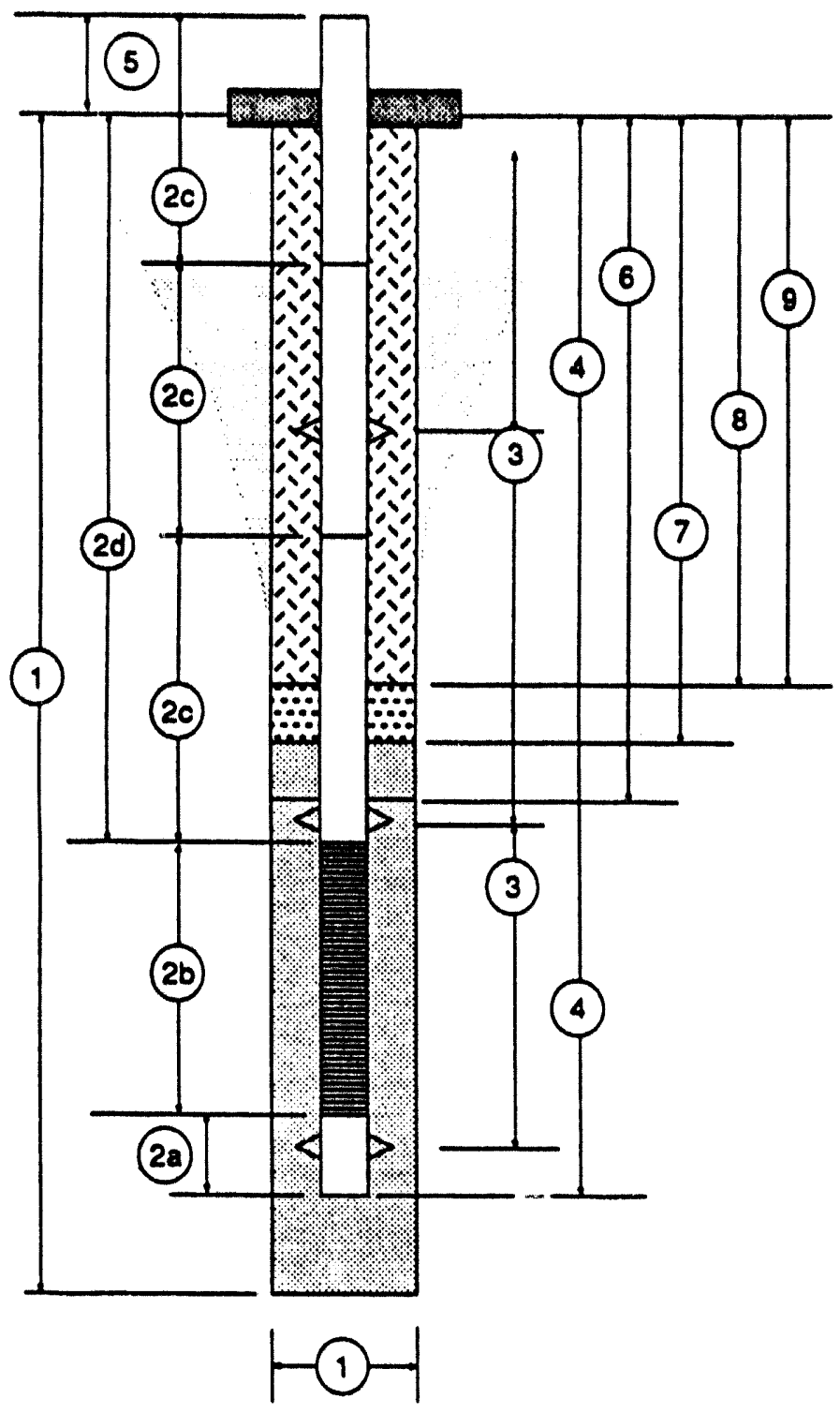

WELL NUMBER MH'- $26^{\top}$ SRS COORDINATES

SANITARY SEAL ELEVATION

NOTE: ALL MEASUREMENTS ARE FROM GROUND SURFACE AT START OF BORING - MEASUREMENTS TO NEAREST 0.1 FOOT.

Fihes Optics Timporature Sinsors

1) Total Drillod Depth/Hole Diameter $53.0^{\prime} / 81 / 2{ }^{\circ \prime}$ 2) Casing/Screen Tally (Messured to Nearest 0.01 Foot)

(a) Sump \& Plug Longth Not reguined

(b) Screen Length Nut reguiced

(c) Casing Joint Lengths (Measured in Uphole Sequenco from Top of Screen) $1 / 10.13^{\circ}$ including theads 2/ $10.00^{\circ}$ 3/ $10.00^{\circ}$ y/ $10.00^{\circ}$ 5/ $9.87^{\circ}$

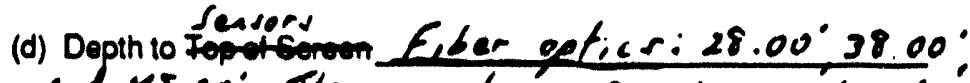
and 48.00 3) Depths to Contralizers Not ceguiced

4) Total Depth of Installed Well $50.0^{\circ}$

5) Casing Stick Up (Standard 2.5' A.G.S.) $2.7^{\circ}$

6) Depth to Top of Fither Pack $9.1^{\circ}$

7) Depth to Top of Fine Sand Seal Not reguired

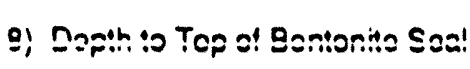
Not reguired

9) Thickness of Grout $9.1^{\circ}$ 


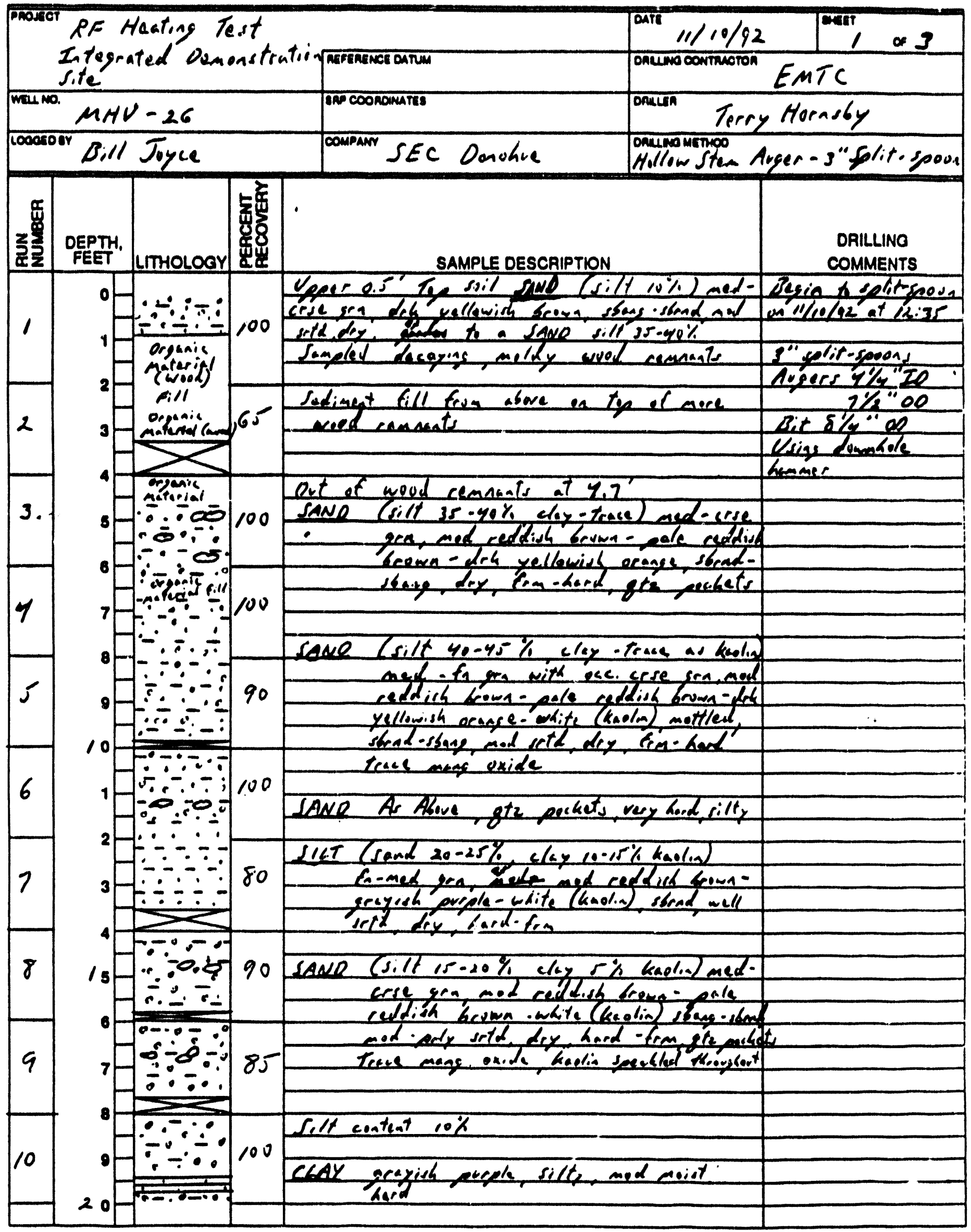




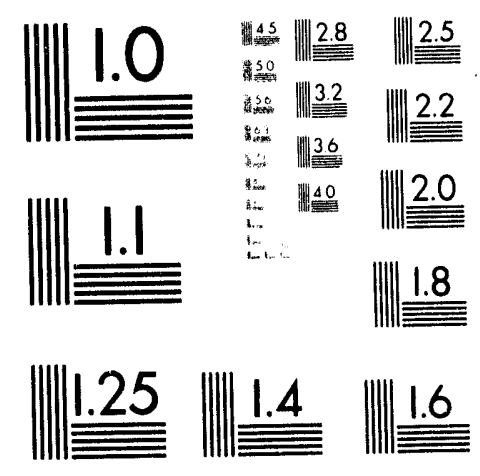




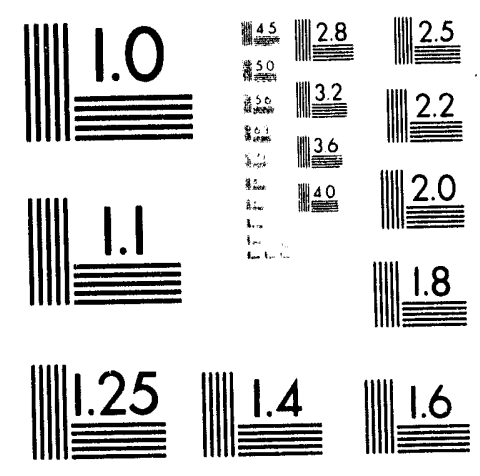



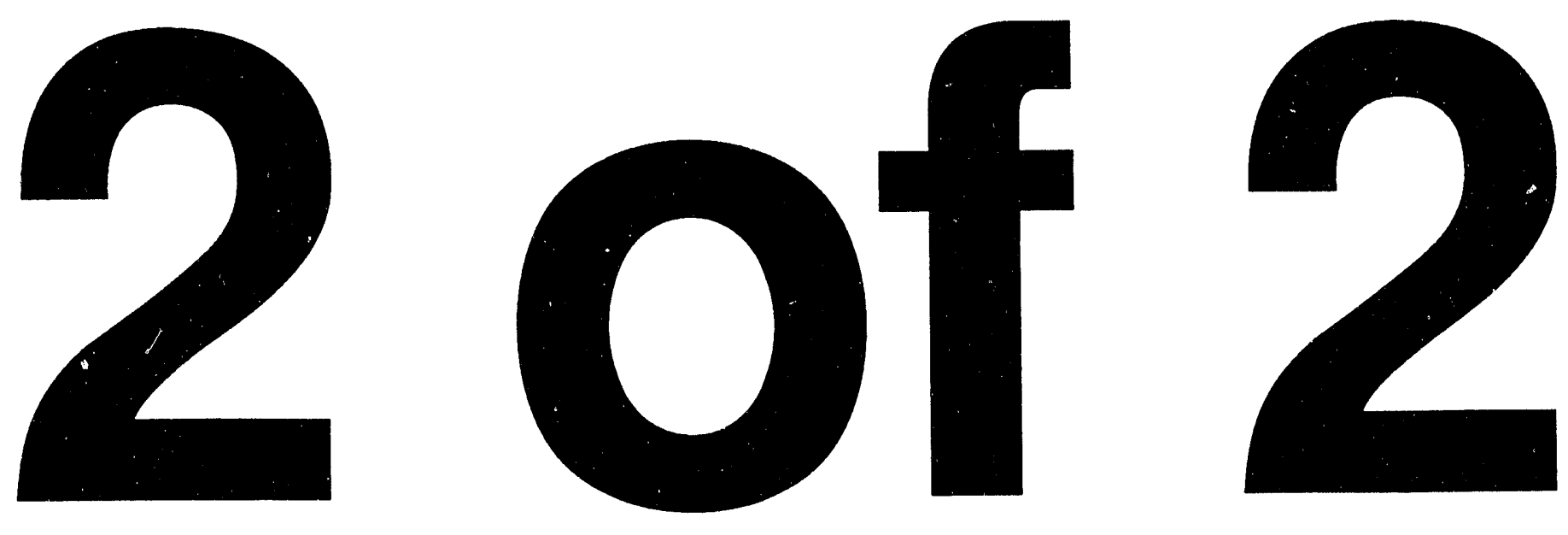
FIELD GEOLOGIC LOG

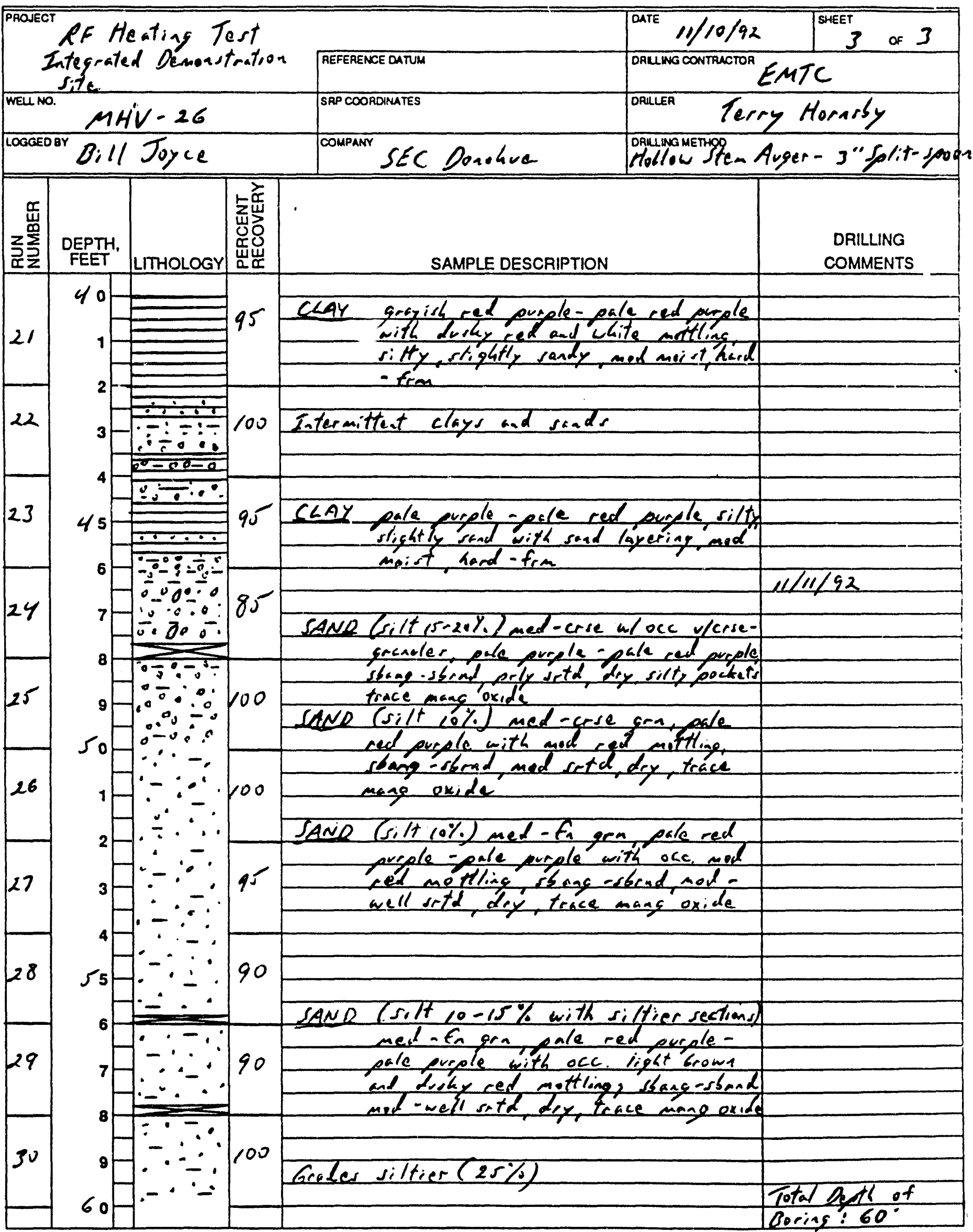


(i) is : $10 \cdot 5$
(lick 12.89

MONITORING WELL CONSTRUCTION DIAGRAM

DRILLING SUBCONTRACTOR E ET

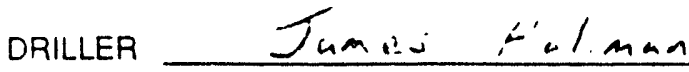

DATE OF WELL INSTALLATION $11 / 25 / \mathrm{C}_{2}$
WELL NUMBER NiMli-i?

SR COORDINATES

SANITARY SEAL ELEVATION TECH. O.S.ICO. NAME Bell Joyce /sEc Dacha

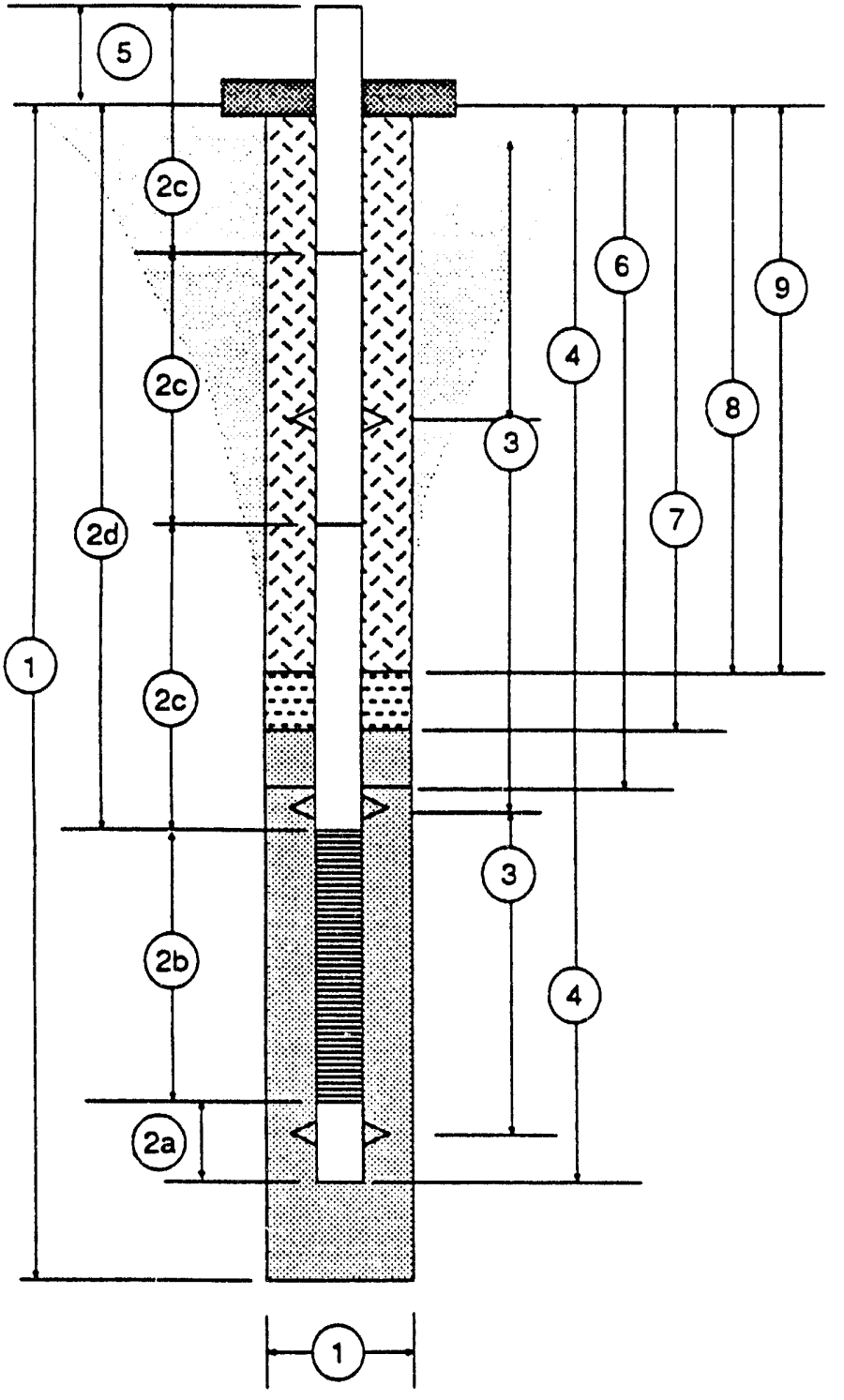

NOTE: ALL MEASUREMENTS ARE FROM GROUND SURFACE AT START OF BORING - MEASUREMENTS TO NEAREST 0.1 FOOT.

1) Total Drilled Depth/Hole Diameter $60.0^{\prime} / 8,4$

2) Casing/Screen Tally (Measured to Nearest 0.01 Foot)

(a) Plug Length $0.0 y^{\prime}$

(b) Screen Length $4.90^{\circ}$

(c) Casing Joint Lengths (Measured in Uphole Sequence From Top of Screen)

9.89

2) 9.92

3) $9.89^{\circ}$

1) 9.93

5) 8.37

(d) Depth to Top of Screen $4 \times 20^{\circ}$

3) Depths to Centralizers Alt cegicied

4) Total Depth of Install $N_{U l l} \ldots \ldots .9^{\circ}$

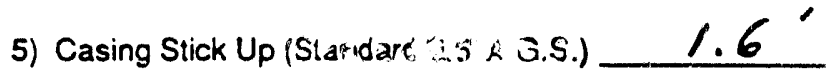

6) Depth to Top of Fine. Pa si _...

7) Depth to Top of Fine Sard it:.._Lat cegeiced

9) Depth to Ton nt Rontnnite Sal _. Not ceguireid

9) Thickness of Grout $2.0^{\circ}$ 
DAILLING SUBCONTRACTOR EMTC

DRILLER Giche Cab.t

DATE OF WELL INSTALLATION $12 / \div 2 / 42$

TECH. O.S.ICO. NAME B.ll/ Joyce/SEC Donuhve

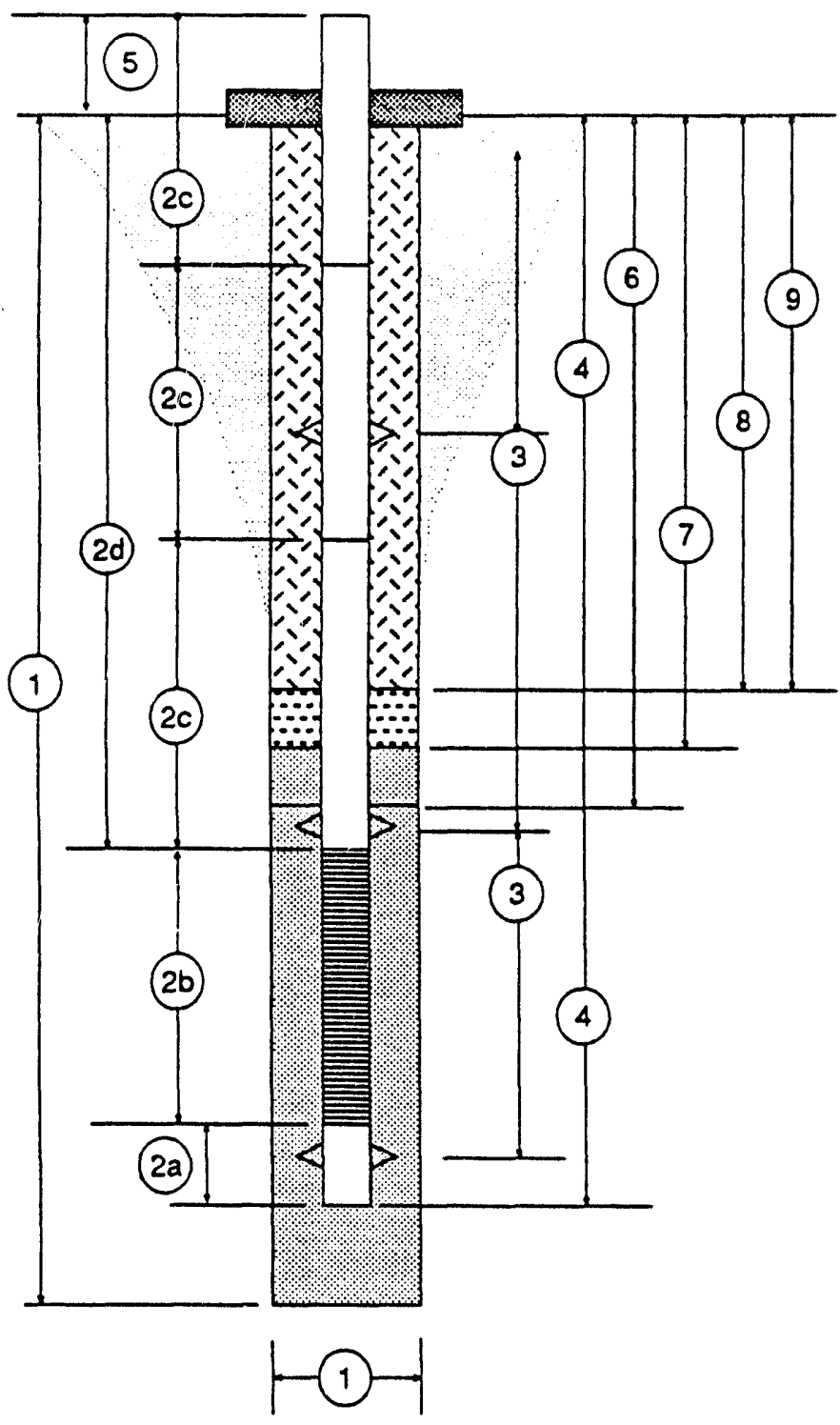

WELL NUMBER_MHL_ $\because ? 3$

SRS COORDINATES

SANITARY SEAL ELEVATION
NOTE: ALL MEASUREMENTS

ARE FROM GROUND

SURFACE AT START

OF BORING - MEASUREMENTS

TO NEAREST 0.1 FOOT.
First Grout
STage

1) Total Drilled Depth/Hole Diameter $43.9 \% 814$

2) Casing/Screen Tally (Measured to Nearest 0.01 Foot)

(a) Plug Length 0.02

(b) Screen Length

4.89

(c) Casing Joint Lengths (Measured in Uphole Sequence From Top of Screen) 2) $9.88^{\circ}$ 3) $9.88^{\circ}$ $\frac{1) 9.92^{\prime}}{4) 8.32^{\prime}}$

(d) Depth to Top of Screen 38.00

3) Depths to Centralizers Not reguired

4) Total Depth of Installed Well $42.9^{\circ}$

5) Casing Stick Up (Standard 2.5' A.G.S.)

6) Depth to Top of Filter Pack 36.2

7) Depth to Top of Fine Sand Seal Not regriced

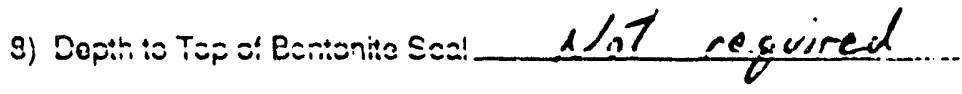

9) Thickness of Grout 2.3 
DRILLING SUBCONTRACTOR EATC

DRILLER

Biake Cab.T

DATE OF WELL INSTALLATION $12 / 03 / i_{2}$
WELL NUMBER_. $M$ H

SRS COORDINATES

SANITARY SEAL ELEVATION

tech. O.s.lco.name Bill Toyce / rec Conuáve

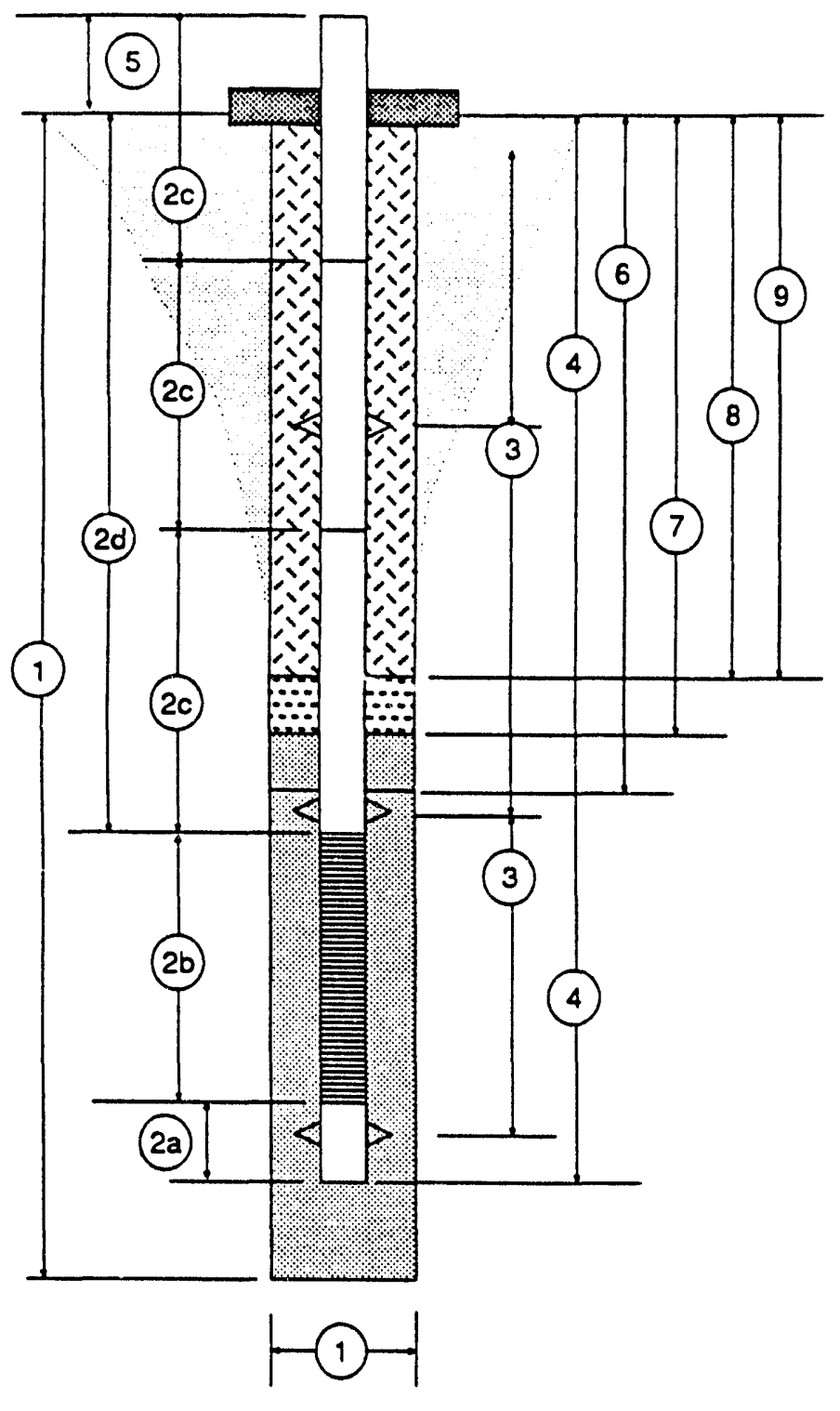

NOTE: ALL MEASUREMENTS

ARE FROM GROUND

SURFACE AT START

OF BORING - MEASUREMENTS

TO NEAREST 0.1 FOOT.

1) Total Drilled Depth/Hole Diameter $\begin{gathered}\text { Secand Grort } \\ \text { Stage } \\ 33.9 \%\end{gathered}$

2) Casing/Screen Tally (Measured to Nearest 0.01 Foot)

(a) Seres Plug Length

$0.02^{\circ}$

(b) Screen Length

$4.90^{\circ}$

(c) Casing Joint Lengths (Measured in Up-

hole Sequence From Top of Screen) 129.88 2) $9.89^{\circ}$ 3) 8.23

(d) Depth to Top of Screen

$28.00^{\circ}$

3) Depths to Centralizers _. Not reguired

4) Total Depth of Installed Well

$32.9^{\circ}$

5) Casing Stick Up (Standard 2.5' A.G.S.)

6) Depth to Top of Fitter Pack $26.1^{\circ}$

7) Depth to Top of Fine Sand Seal Not reguired

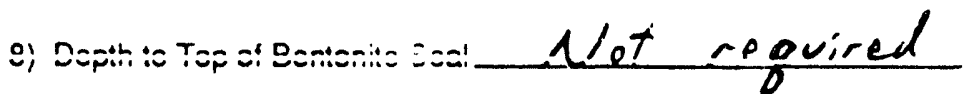

9) Thickness of Grout 26.1 
PAGE 1 OF 2

SEC DONAHUE

CALCULATION SHEET

CLIENT WSRC LOCATION SR JOB NO. G. 4346 SUBJECT RF Heating Test - MHV - 27 Piezometer Well Construction Diagram BY" Bill Joyce DATE $12 / 03 / 92$ CHECKED BY DATE

From

Bottom

Casing

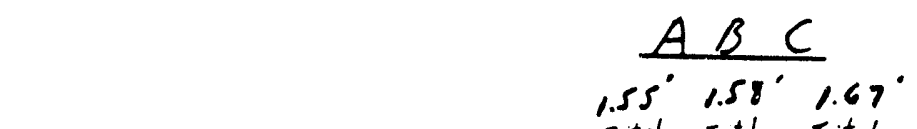

$$
M H V-27
$$

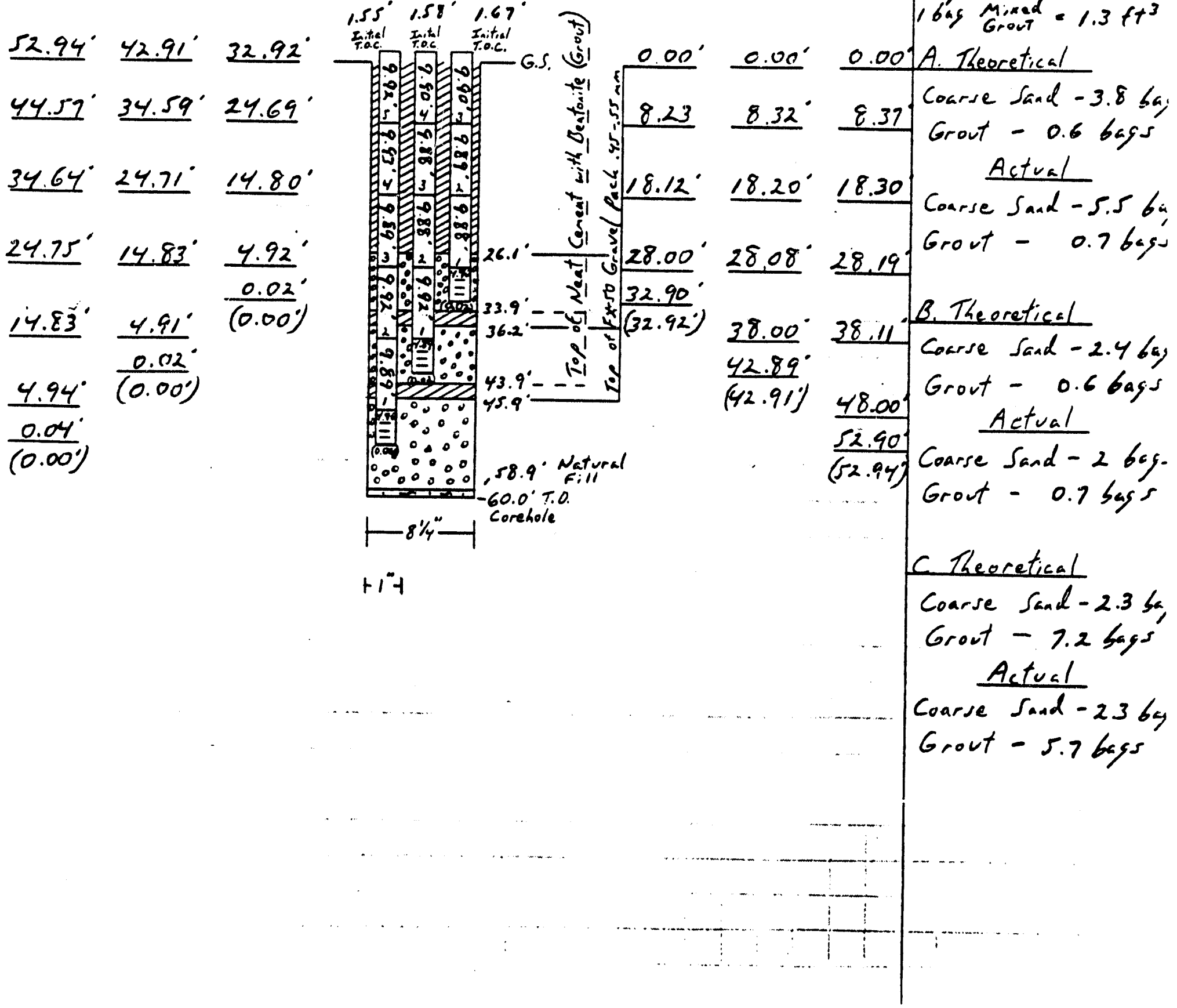

from

Ground

Surface
Gravel Pack

Based on Practical

Application in the Field of ital Coarse Sand $=1.2 \mathrm{ft}^{3}$

bag Grot t $=0.6 \mathrm{ft}^{3}$

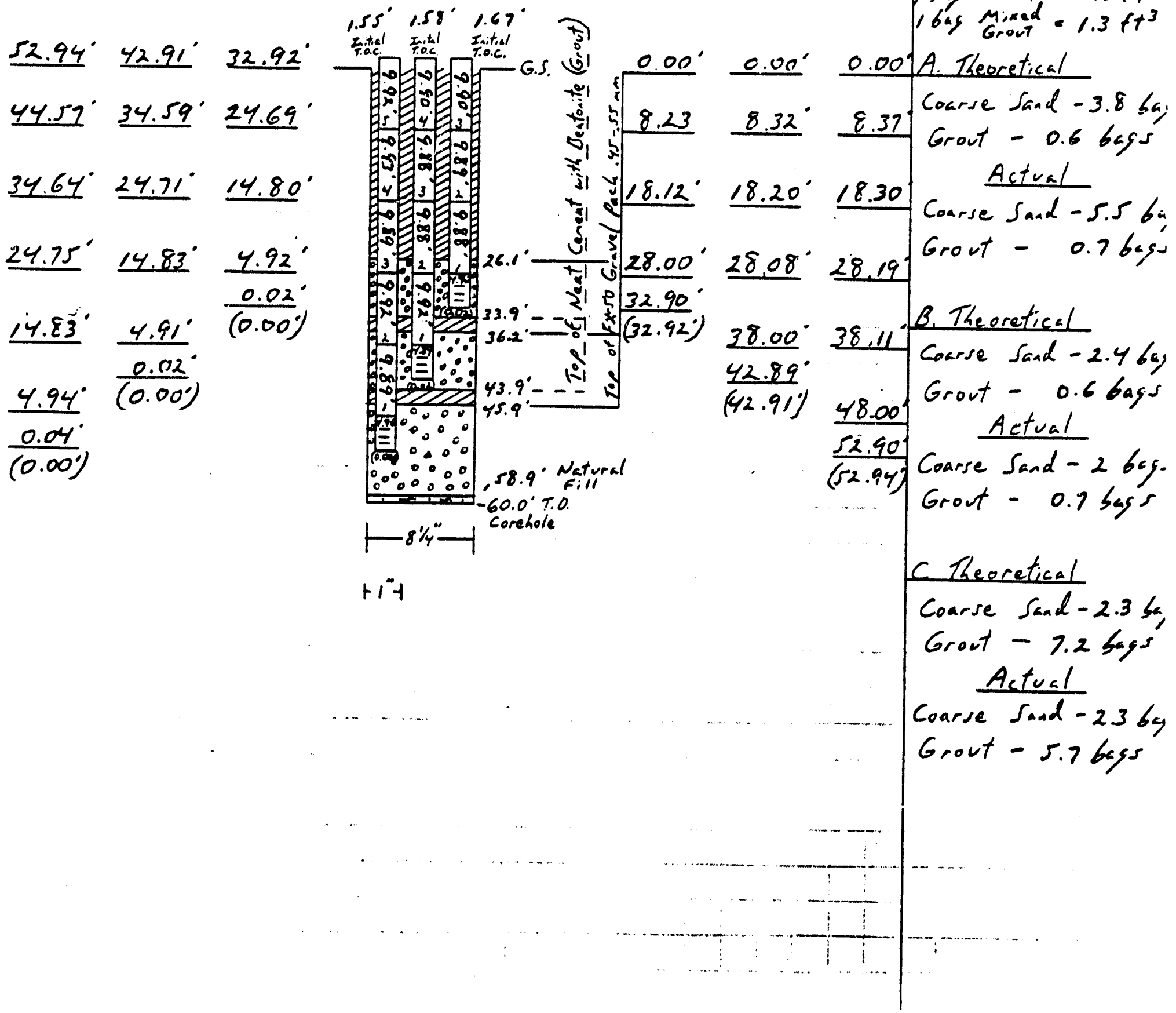


PAGE 2 of 2

SEC DONOHUE

CLIENT

WSRC
JOB NO. G4346

Piezometers Casing Tally DATE

Screen/Plig

Tally

A.

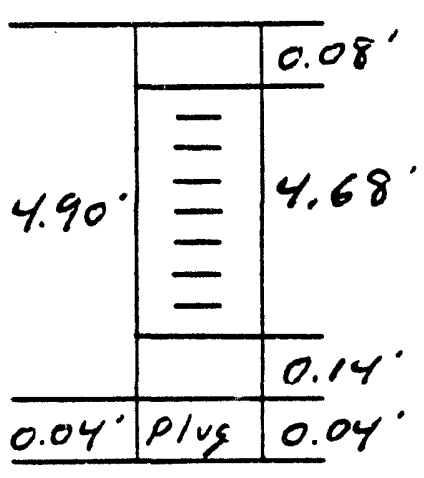

$\frac{\text { Screen }}{1 " \text { PVC }}$

slotted

0.010 in. slot

Flush

Tri-Loc Threads

Timco Grand

Sump

Not required

plug

B

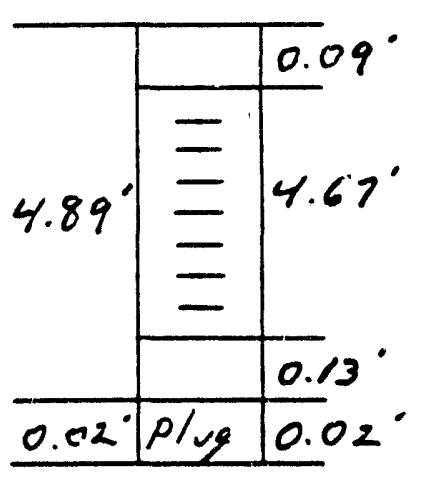

Schedule yo

Tri-Luc Treads

Flush

Tinco Brand c

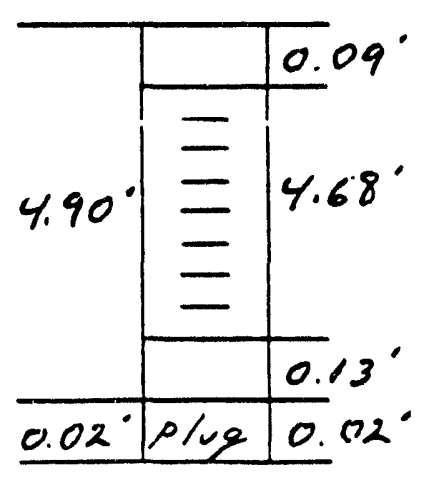




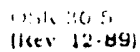

MONITORING WELL CONSTRUCTION DIAGRAM

DRILLING SUBCONTRACTOR EMT

DRILLER Garry Moire/ Steve Reese

DATE OF WELL INSTALLATION $1 / 22 / 93$
WELL NUMBER MトVー27T

SRO COORDINATES

SANITARY SEAL ELEVATION

NOTE: ALL MEASUREMENTS

ARE FROM GROUND

SURFACE AT START

OF BORING - MEASUREMENTS

TO NEAREST 0.1 FOOT.

tech. os. con name Bill Joyce / SEC Donuhue

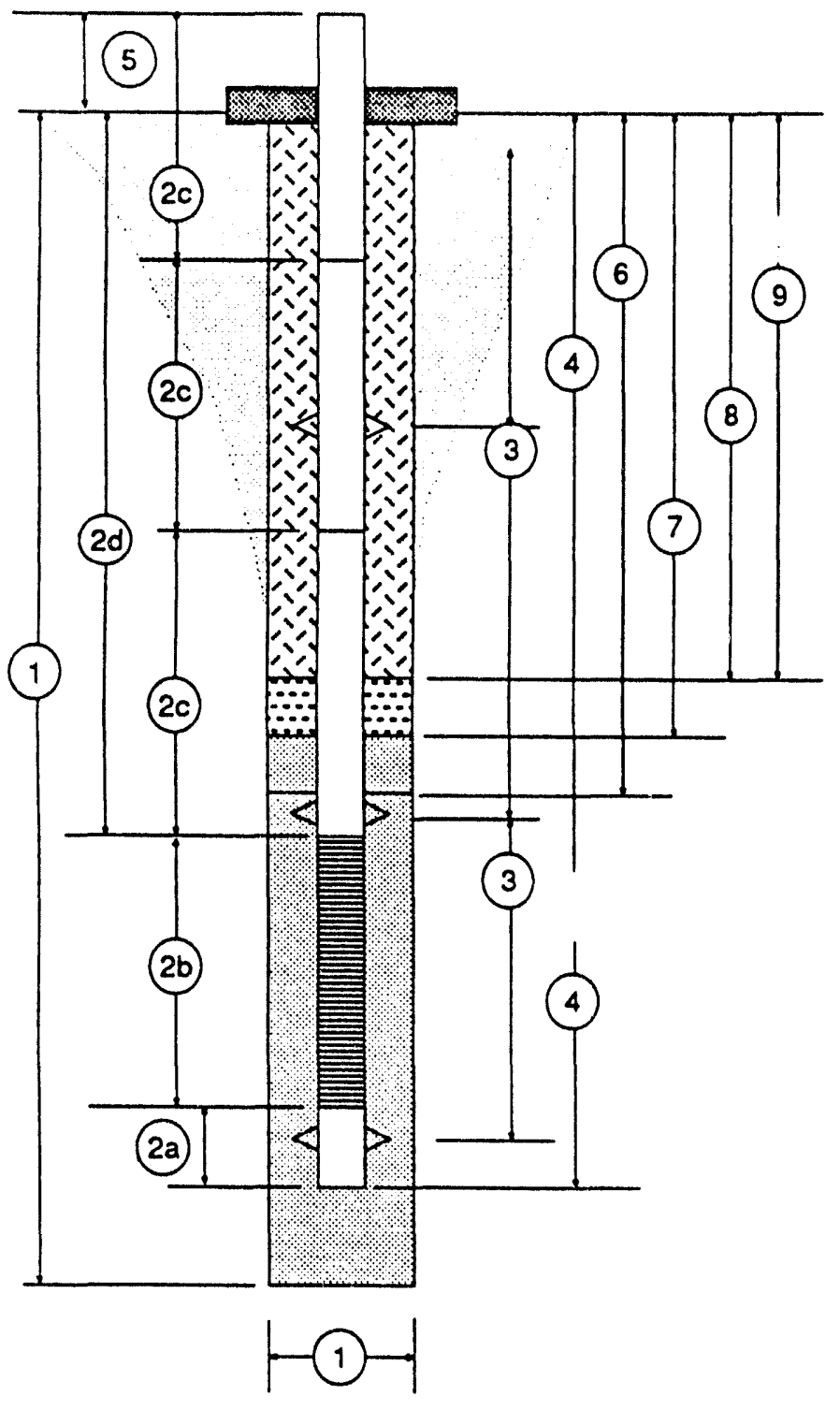

Finer Optics Temperature Sensors

1) Total Drilled Depth Hole Diameter $58.0^{\circ} / 8 \%$

2) Casing/Screen Tally (Measured to Nearest 0.01 Foot)

(a) Sump \& Plug Length Not required

(b) Screen Length Not re quire

(c) Casing Joint Lengths (Measured in Uphole Sequence From Top of Screen) _ 10.13 including threads of 10.00 3) 10.00 4) 9.99 5) 9.88

(d) Depth to fopopen Fiber optics: $28.00^{\circ}, 38.00^{\circ}$ and 48.00: Thermocouples: $28.00^{\circ}, 38.00^{\circ}$ and 3) Depths to Centralizers Not cegrired

4) Total Depth of Installed Well $50.0^{\circ}$

5) Casing Stick Up (Standard 2.5' A.G.S.) $2.6^{\prime}$

1. Depth to Top of Filter Pack 9.5

7) Depth 10 Top of Fine Sand Seal__ Not required

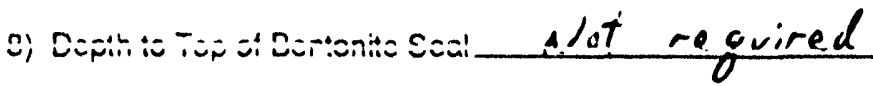

9) Thickness of Grout

$9.5^{\circ}$ 
FIELD GEOLOGIC LOG

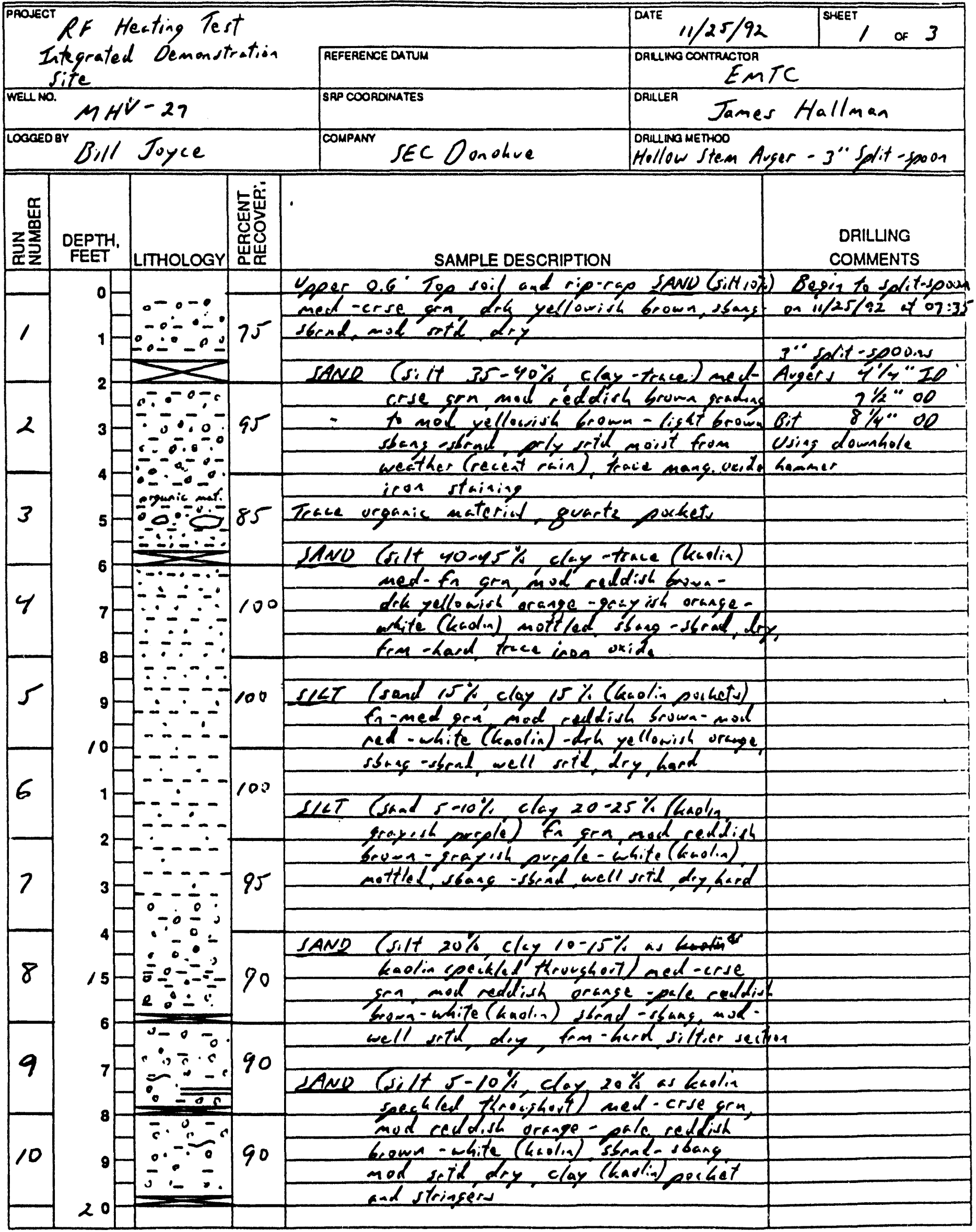


FIELD GEOLOGIC LOG

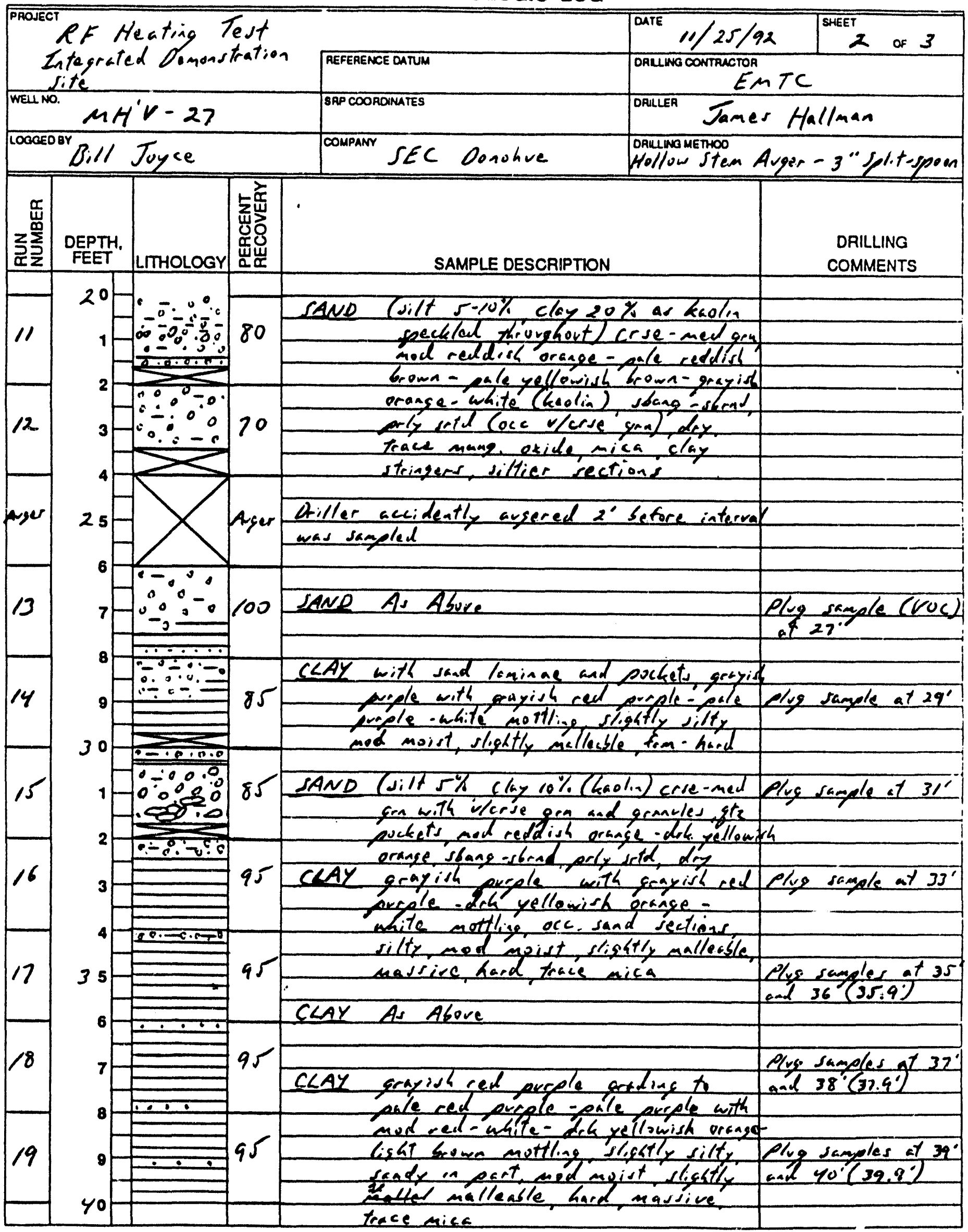


FIELD GEOLOGIC LOG






\section{MONITORING WELL CONSTRUCTION DIAGRAM}

DRILLING SUBCONTRACTOR ENTC

DRILLER Eialse Cab.

WELL NUMBER $\Lambda^{\prime} \kappa^{\prime} / 2 \leq . \Lambda$

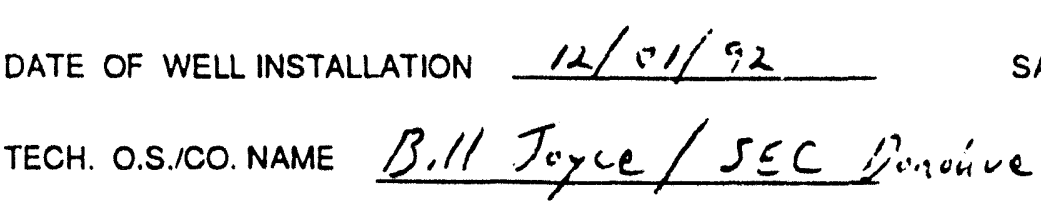

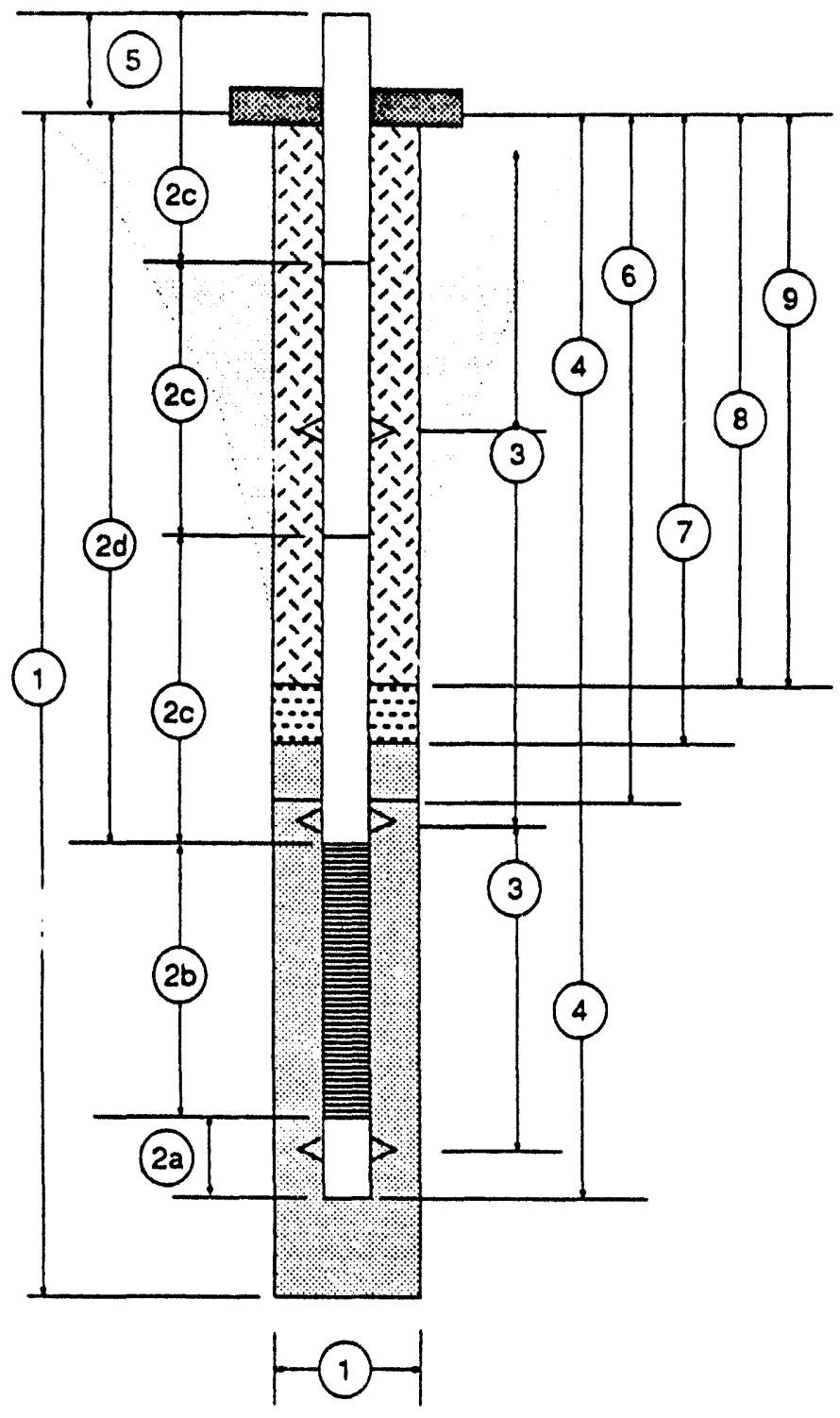

NOTE: ALL MEASUREMENTS

ARE FROM GROUND

SURFACE AT START

OF BORING - MEASUREMENTS

TO NEAREST 0.1 FOOT.

1) Total Drilled Depth/Hole Diameter_ $60.0^{\circ} / 81 / 4 "$

2) Casing/Screen Tally (Measured to Nearest 0.01 Foot)

(a) Plug Length

$0.02^{\circ}$

(b) Screen Length

4.89

(c) Casing Joint Lengths (Measured in Uphole Sequence From Top of Screen) 1) $9.90^{\circ}$ 2) $9.90^{\circ}$ 3) $9.89^{\circ} 4 / 9.88^{\circ}$ 5) 8.43

(d) Depth to Top of Screen

$48.00^{\circ}$

3) Depths to Centralizers

Not reguired

4) Total Depth of Installed Well

$52.9^{\circ}$

5) Casing Stick Up (Standard 2.5' A.G.S.)

$1.5^{\prime}$

6) Depth to Top of Fiter Pack

$46.2^{\prime}$

7) Depth to Top of Fine Sand Seal

Not reguired

8! Dopth ! Tsant gon!nnito Soal_ Not reguired.

9) Thickness of Grout 2.3 
DRILLING SUBCONTRACTOR EMTC DRILLER Bianc Cabi:
WELL NUMBER_M $M L^{\prime}=\varepsilon \mathscr{E}$ SRS COORDINATES

SANITARY SEAL ELEVATION TeCH. O.S.ICO.name Bill Toy-e/SEC Oonohue

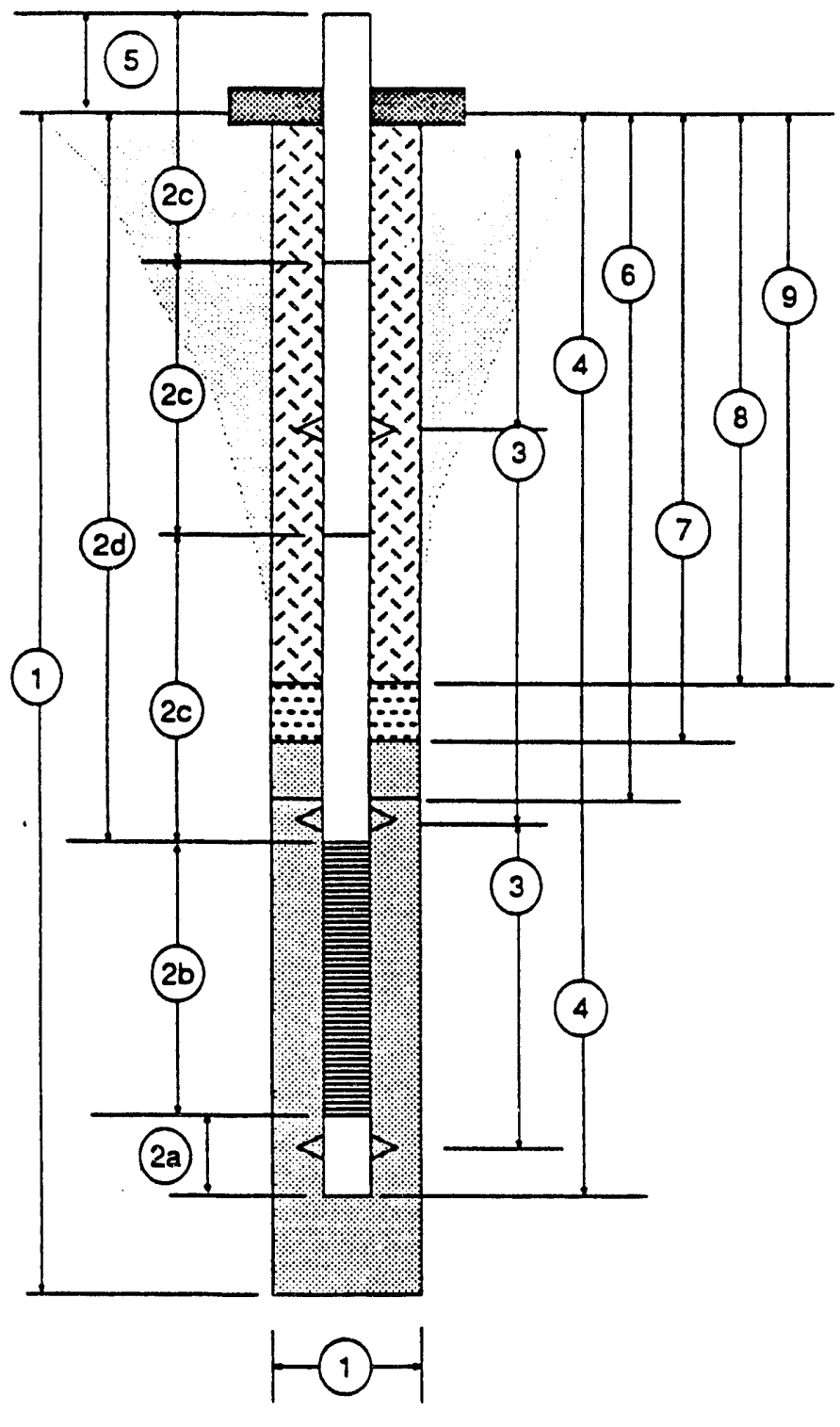

NOTE: ALL MEASUREMENTS

ARE FROM GROUND

SURFACE AT START

OF BORING - MEASUREMENTS

TO NEAREST 0.1 FOOT.

First Gru.t
Stage
$43.9^{\circ} / 81 / 4 "$

2) Casing/Screen Tally (Measured to Nearest 0.01 Foot)

(a) Plug Length

0.02

(b) Screen Length

$4.90^{\circ}$

(c) Casing Joint Lengths (Measured in Uphole Sequence From Top of Screen) 1) $9.91^{\prime}$ 2) $9.90^{\circ}$ 3) $9.90^{\circ}$ 4) $8.29^{\circ}$

(d) Depth to Top of Screen $38.00^{\circ}$

3) Depths to Centralizers Not reguired

4) Total Depth of Installed Well $42.9^{\prime}$

5) Casing Stick Up (Standard 2.5' A.G.S.) $1.6^{\circ}$

6) Depth to Top of Filter Pack $34.9^{\prime}$

7) Depth to Top of Fine Sand Seal Not reguired

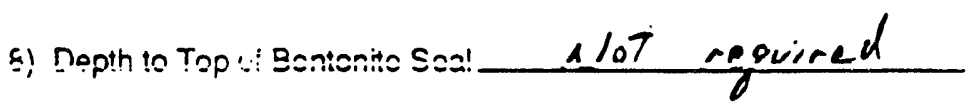

9) Thickness of Grout $1.5^{\circ}$ 
DRILLING SUBCONTRACTOR EMTC

DRILLER Biake Cabit

DATE OF WELL INSTALLATION $12 / 03 / 92$ TECH. O.S.ICO. name D.ll Joycel/SEC Donchue

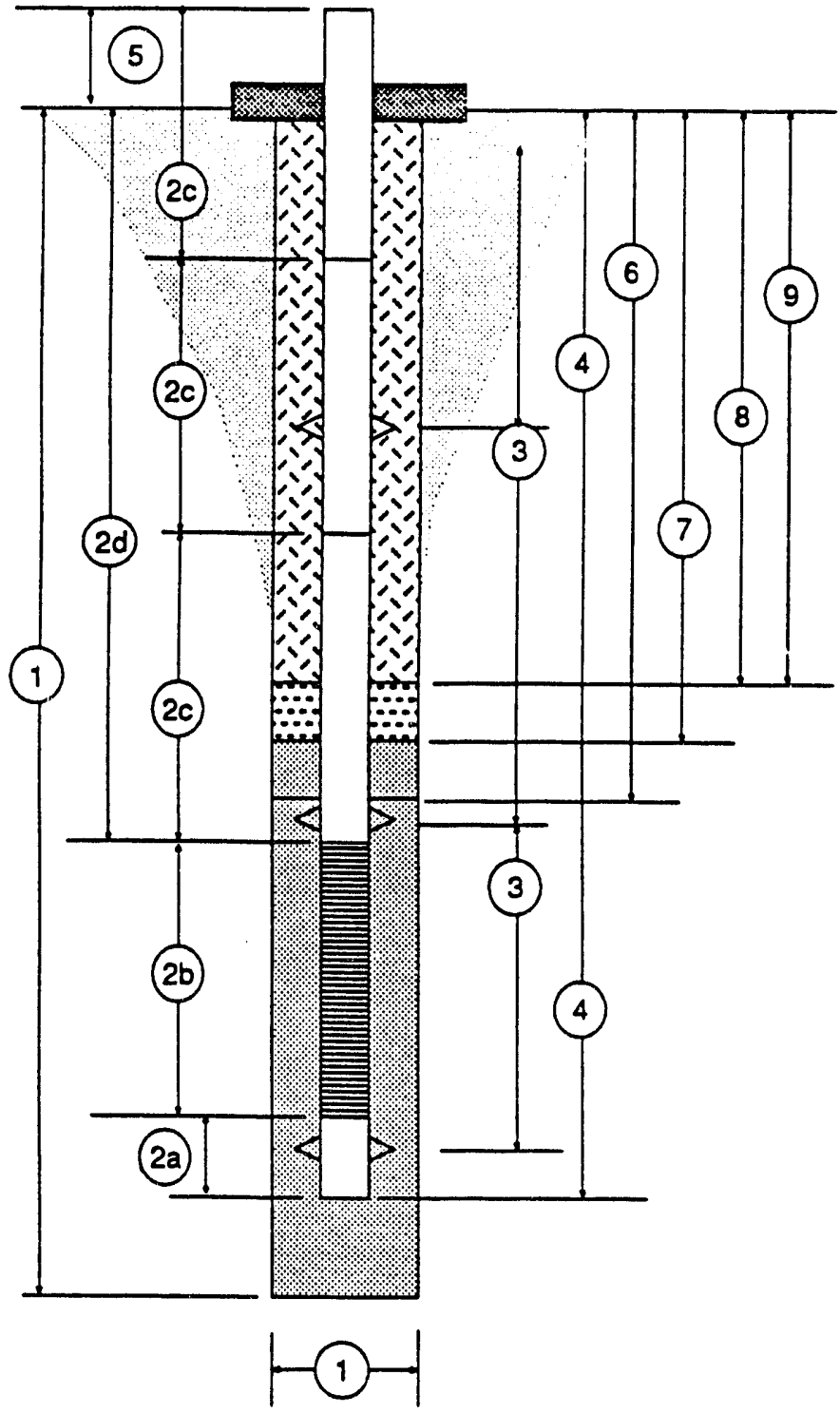

WELL NUMBER MTHV.28C

SRS COORDINATES

SANITARY SEAL ELEVATION

NOTE: ALL MEASUREMENTS

ARE FROM GROUND

SURFACE AT START

OF BORING - MEASUREMENTS

TO NEAREST 0.1 FOOT.

1) Total Drilled DepthHole Diameter $\begin{gathered}\text { Secand Gront } \\ \text { stume } \\ 33.4 / 81 / 4 "\end{gathered}$

2) Casing/Screen Tally (Measured to Nearest 0.01 Foot)

(2) Plug Length

0.02

(b) Screen Length

4.89

(c) Casing Joint Lengths (Measured in IJphole Sequence From Top of Screen) 1) $9.91^{\circ}$ 2) $9.89^{\circ}$ 3) $8.20^{\circ}$

(d) Depth to Top of Screen 28.00

3) Depths to Centralizers Not reguired

4) Total Depth of Installed Well $32.9^{\prime}$

5) Casing Stick Up (Standard 2.5' A.G.S.) 1.7

6) Depth to Top of Fitter Pack 25.6

7) Depth to Top of Fine Sand Seal _Not required

8) Donth in Ton of Bentonite Sea! Alot re guirced

9) Thickness of Grout $25.6^{\prime}$ 
PAGE

of 2

SEC DONAHUE

CALCULATION SHEET

CLIENT WSAC LOCATION SR JOB NO. G. 4346 SUBJECT RF Heating Test - MHV - 28 Piezometer Well Construction Diagram $\mathrm{BY}^{--}$Bill Joyce DATE $12 / 03 / 42$ CHECKED BY DATE

From.

Bottom

Casing

$$
M H V-28
$$

From

Ground

Surface
Gravel Pack

Based on Practical

Application in the field of

1 bag Coarse Sand $=1.2 \mathrm{ft}^{\circ}$

bag Gory t $=0.6 \mathrm{ft}^{3}$

bog Mixed $=1.3 \mathrm{ft}$ :

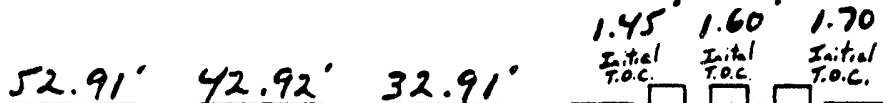

$0.00^{\prime} 0.00^{\circ} 0.001$ A. Theoretical $44.48^{\circ} 34.63^{\circ} 24.71^{\circ}$

$34.60^{\circ} \quad 24.73^{\circ} \quad 14.82^{\circ}$

$24.1^{\circ}$
$14.81^{\circ}$$\frac{14.83^{\circ}}{4.92^{\circ}} \frac{4.91^{\circ}}{\frac{0.02}{0.00^{\circ}}}$

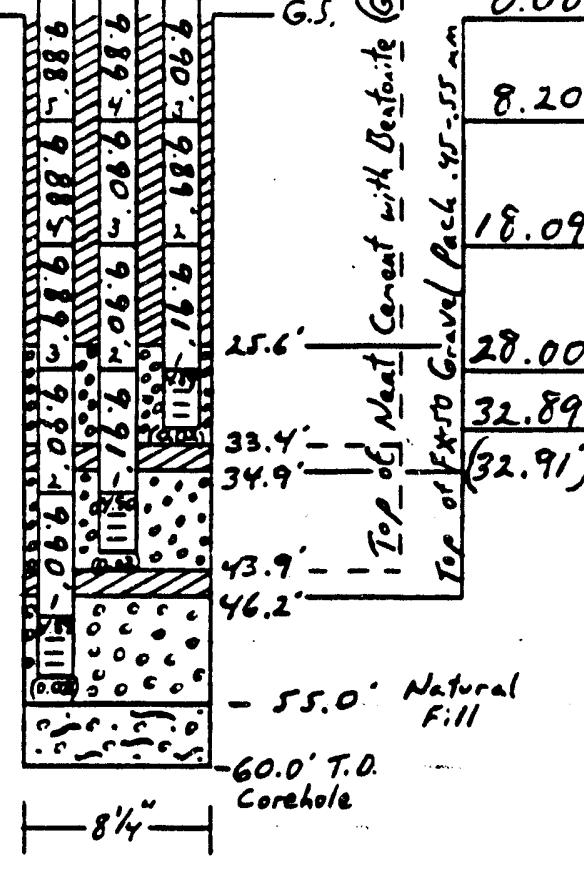

$+14$ Coarse Sand - 2.66 . Grout - 0.6 bags

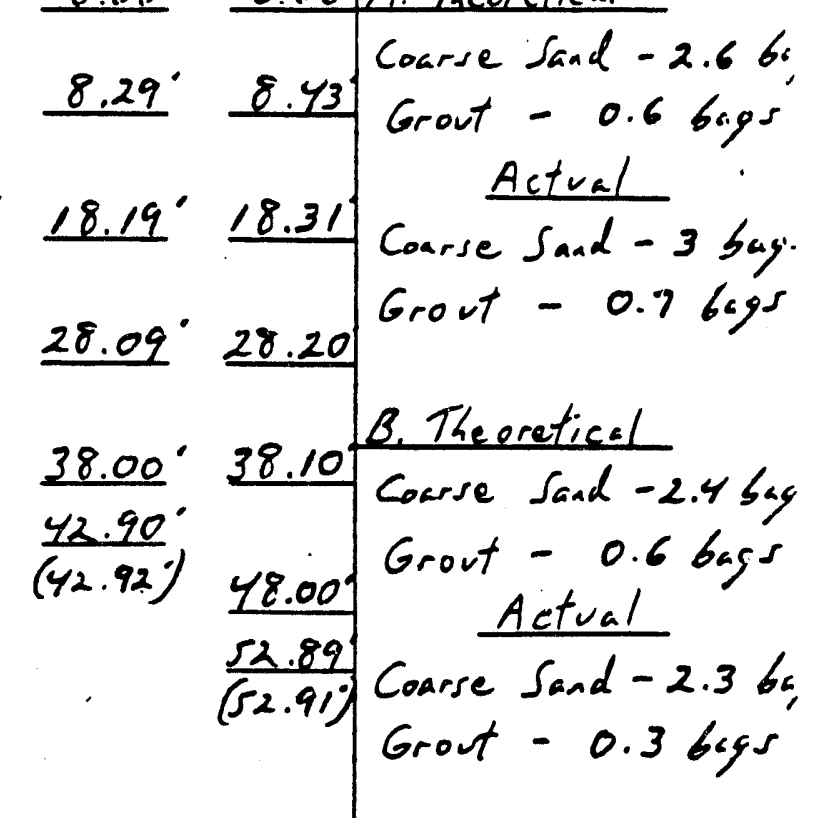

c Theoretical Coarse Sand - 2.3 b: Grout - 7.2 bags Actual Coarse Sand - 2.5 bay Grout - 4.7 bags 
PAGE 2 OF 2

SEC DONAHUE

CALCULATION SHEET

CLIENT WSRC LOCATION SR S JOB NO. G 4346 SUBJECT RF Heating Test - MHV - $2 \hat{8}$ Piezometers Casing Tally BY Bill Joyce DATE $12 / 03 / 9,2$ CHECKED BY DATE

$\frac{\text { Casing }}{\text { Tally }} \frac{\begin{array}{l}\text { Screen } \\ \text { 1) } \frac{A}{9.90^{\circ}}\end{array}}{\frac{A}{48.0^{\circ}-53.0^{\circ}}}$

2) $9.90^{\circ}$

3) 9.89

4) 9.88

5) $\frac{9.88^{\circ}}{49.45^{\circ}}$

- 48.00 Top of Casing

$1.45^{\circ}$ stickup

1) $\frac{B}{9.91^{\circ}} \quad \frac{B}{38.0^{\circ}-43.0^{\circ}}$

2) $9.90^{\circ}$

3) $9.90^{\circ}$

4) $\frac{9.89^{\circ}}{39.60^{\circ}}$

$-38.00^{\circ}$ Top of Cawing $1.60^{\circ}$ Stickup

1) $\frac{C}{9.91^{\circ}} \quad \frac{C}{28.0^{\circ}-33.0^{\circ}}$

2) $9.89^{\circ}$

3) $\frac{9.90^{\circ}}{29.70^{\circ}}$

- 28.00' Top of Casing $1.70^{\circ}$ sticks
Materials

List $(A, B C)$

$\frac{\text { Casing }}{\text { "PvC }}$

Schedule yo

Tri-Loc Threads

Flush

Tinco Brand

$\frac{\text { Screen }}{\text { "PVC }}$

slotted

$0.010 \mathrm{in}$. slot

Flush

Tri-Loc Threads

Times Brand

Sump

Not required

plug

I"puc

Schedule yo

Tri-Luc Treads

Flush

Timco Brand
Screen / Plug

Tally

A

\begin{tabular}{l|l|l}
\hline & & $0.09^{\circ}$ \\
\cline { 2 - 3 } & & \\
$4.89^{\circ}$ & $\equiv$ & $4.66^{\circ}$ \\
& $\equiv$ & \\
\cline { 2 - 3 } & & $0.14^{\circ}$ \\
\hline $0.02^{\circ}$ & $\rho \operatorname{lug}$ & $0.02^{\circ}$ \\
\hline
\end{tabular}

B

\begin{tabular}{l|l|l}
\hline & & $0.08^{\circ}$ \\
& $=$ & \\
$4.90^{\circ}$ & $=$ & $4.69^{\circ}$ \\
& $=$ & \\
& & \\
\cline { 2 - 2 } & $0.13^{\circ}$ \\
\hline 0.02 & plug & 0.02 \\
\hline
\end{tabular}

c

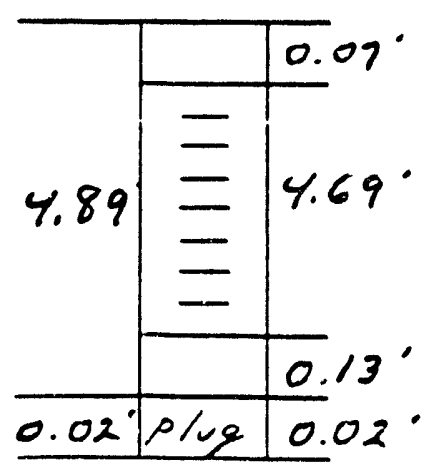


DRILLING SUBCONTRACTOR $E M T C$

DRILLER Garry Miore DATE OF WELL INSTALLATION $1 / 15 / 93$ TECH. O.S.ICO.NAME D.ll Toyce/SEC Donohoe

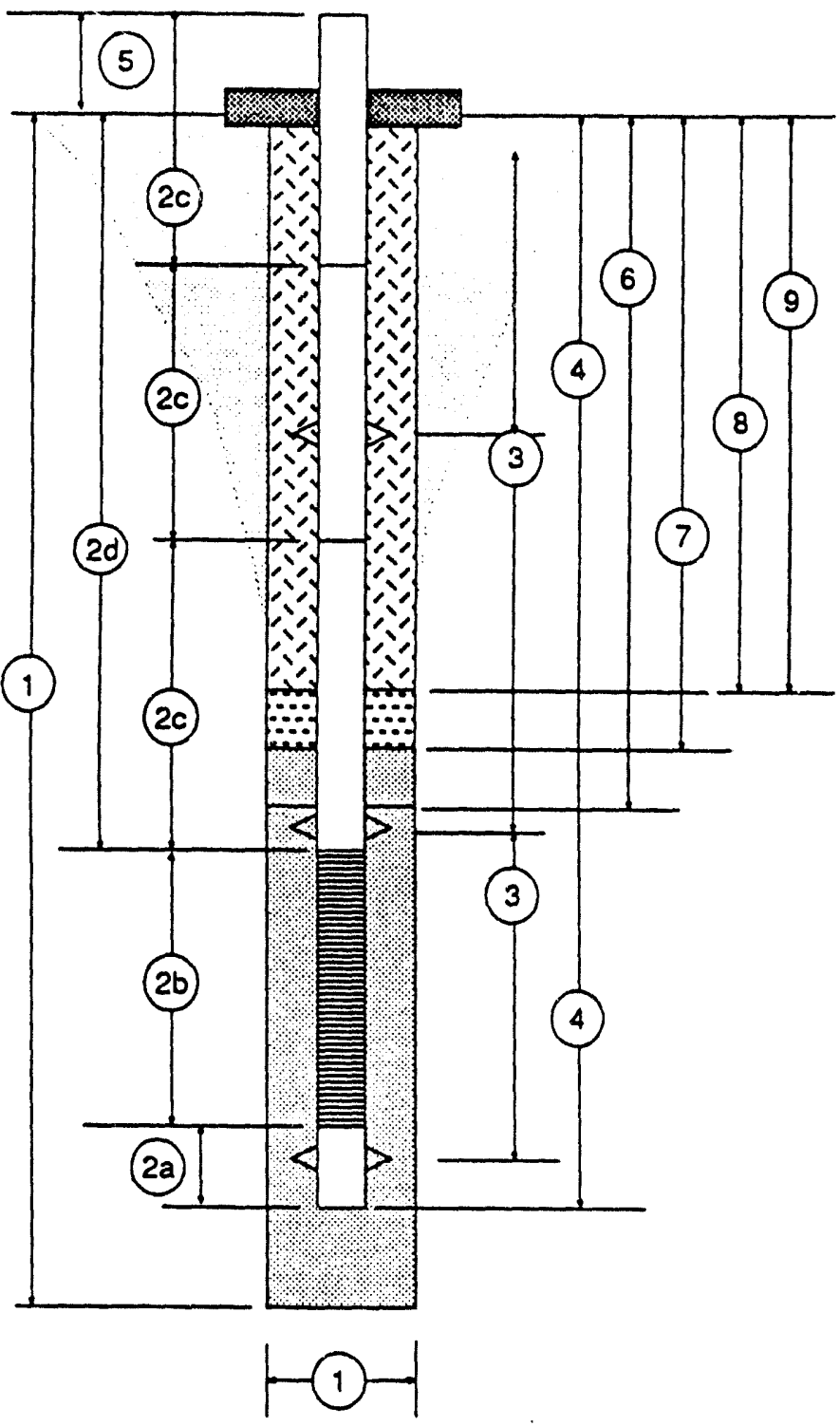

WELL NUMBER M MUV $=\varepsilon T$ SRS COORDINATES

SAINITAFIY SEAL ELEVATION
NOTE: ALL MEASUREMENTS

ARE FROM GROUND

SURFACE AT START

OF BORING - MEASUREMENTS

TO NEAREST 0.1 FOOT.

Fiber Optices Temperature Seasors

1) Total Drilled Depth/Hole Cameter $58.0^{\prime} / 81 / 2 "$

2) Casing/Screen Tally (Measured to Nearest 0.01 Foot)

(a) Sump \& Plug Length

Net reguired

(b) Screen Length Not ceguired

(c) Casing Joint Lengths (Measured in Uphole Sequence From Top of Screen) 1) $10.12^{\circ}$ including thio.ads 2) $10.00^{\circ}$ 3) $10.00^{\circ}$ \& $10.00^{\circ} 5 / 2.88^{\circ}$

(d) Depth to Seapors Fiber optics: $28.00^{\circ} 38.00^{\circ}$

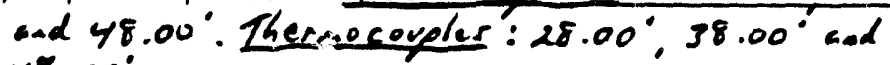
$48.00^{\circ}$

3) Depths to Centralizers Not reguired

4) Total Depth of Installed Well 50.0

5) Casing Stick Up (Standard 2.5' A.G.S.) Z Z.6

6) Depth to Top of Filfer Pack $10.5^{\prime}$

7) Depth to Top of Fine Sand Seal Not regriced

D) Depiil iu Tup vi ōerllunite jear ítit reguireá

9) Thickness of Grout $10.5^{\circ}$ 


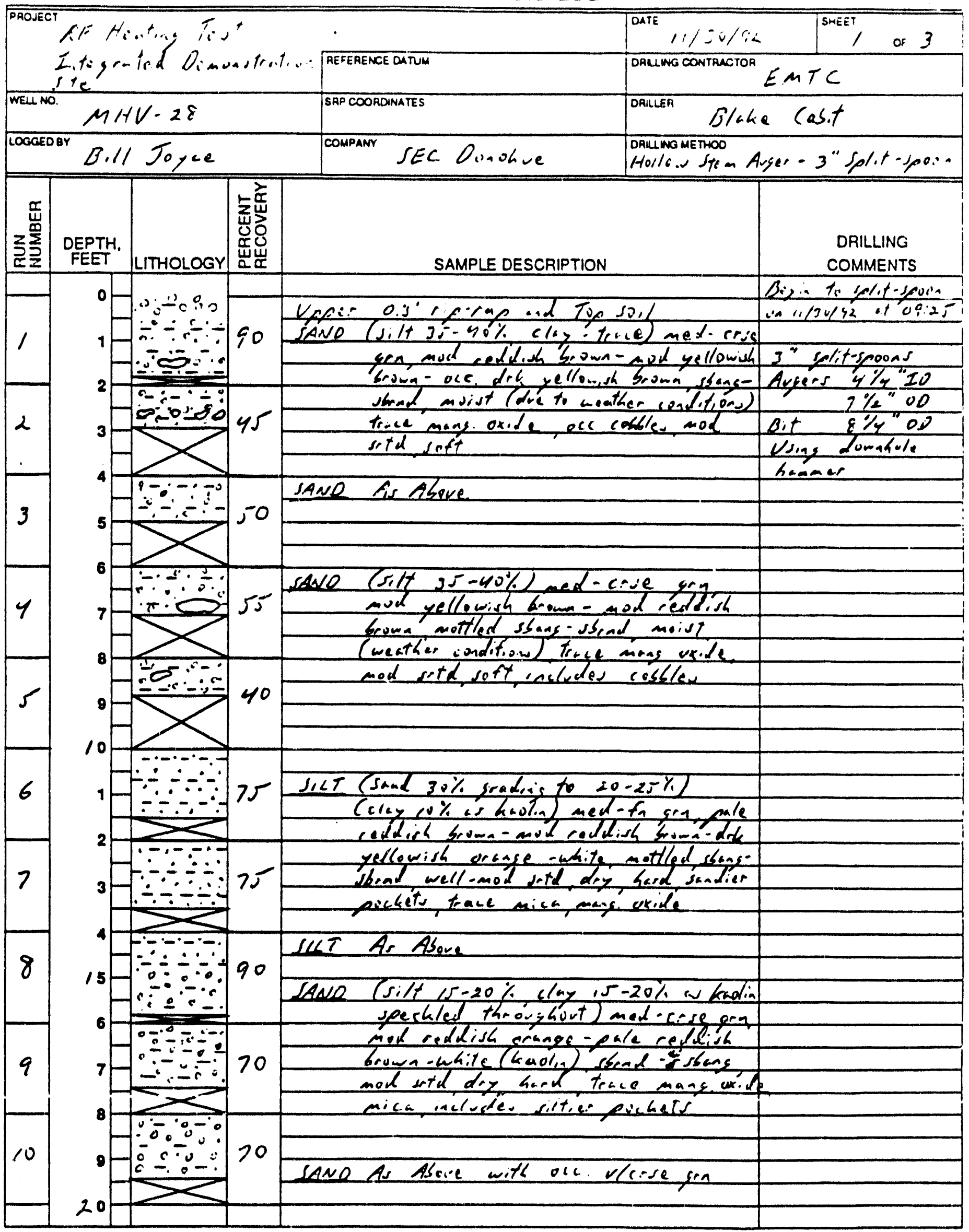


FIELD GEOLOGIC LOG

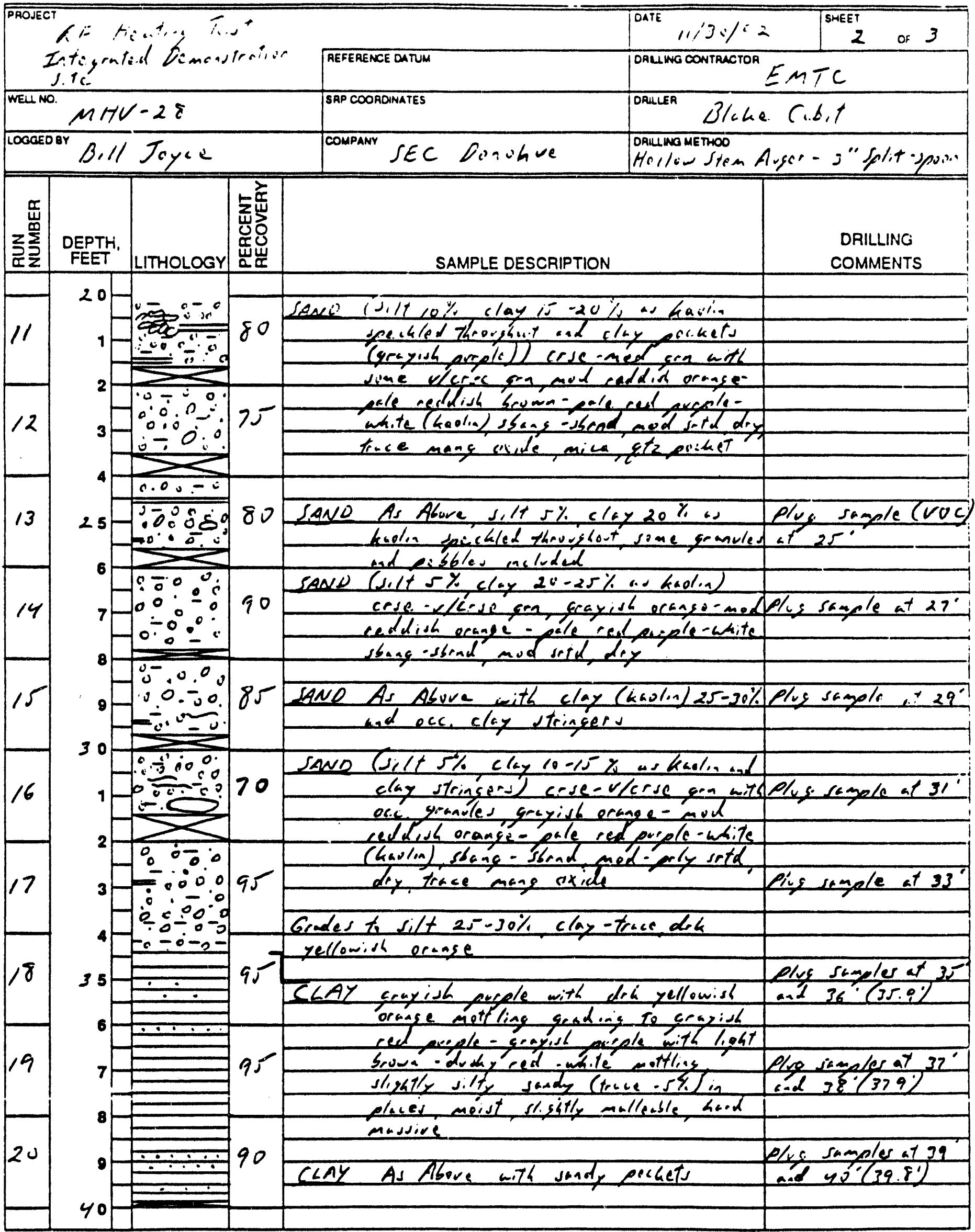


FIELD GEOLOGIC LOG

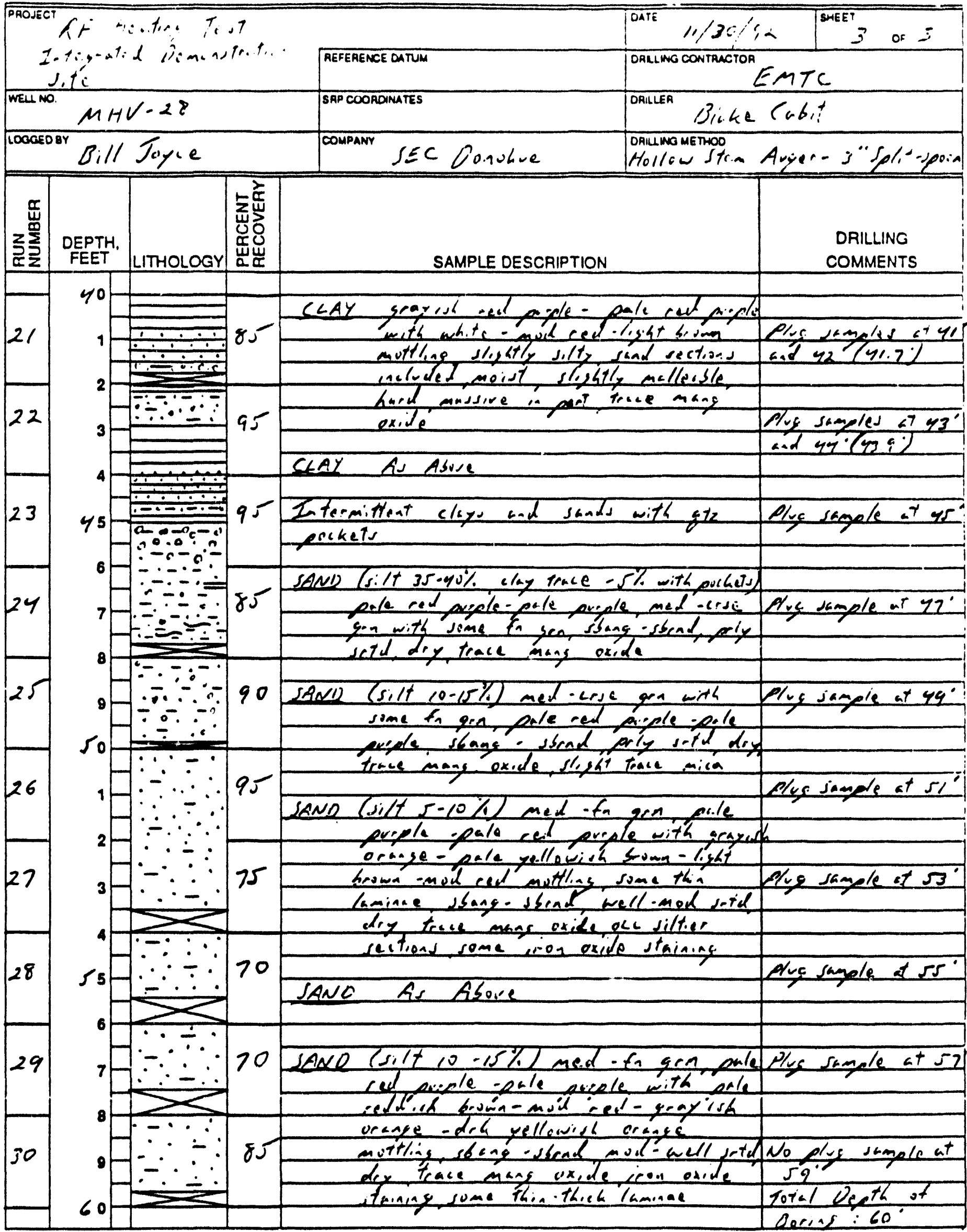


DRILLING SUBCONTRACTOR ENTE

DRILLER STIGL: C Cab,-

DATE OF WELL INSTALLATION $11 / 24 / 92$
WELL NUMBER $M \sim V \cdot 2: A$

SRS COORDINATES

SANITARY SEAL ELEVATION

TECH. O.S.ICO. NAME B.ll Joywe / SEC Conohue

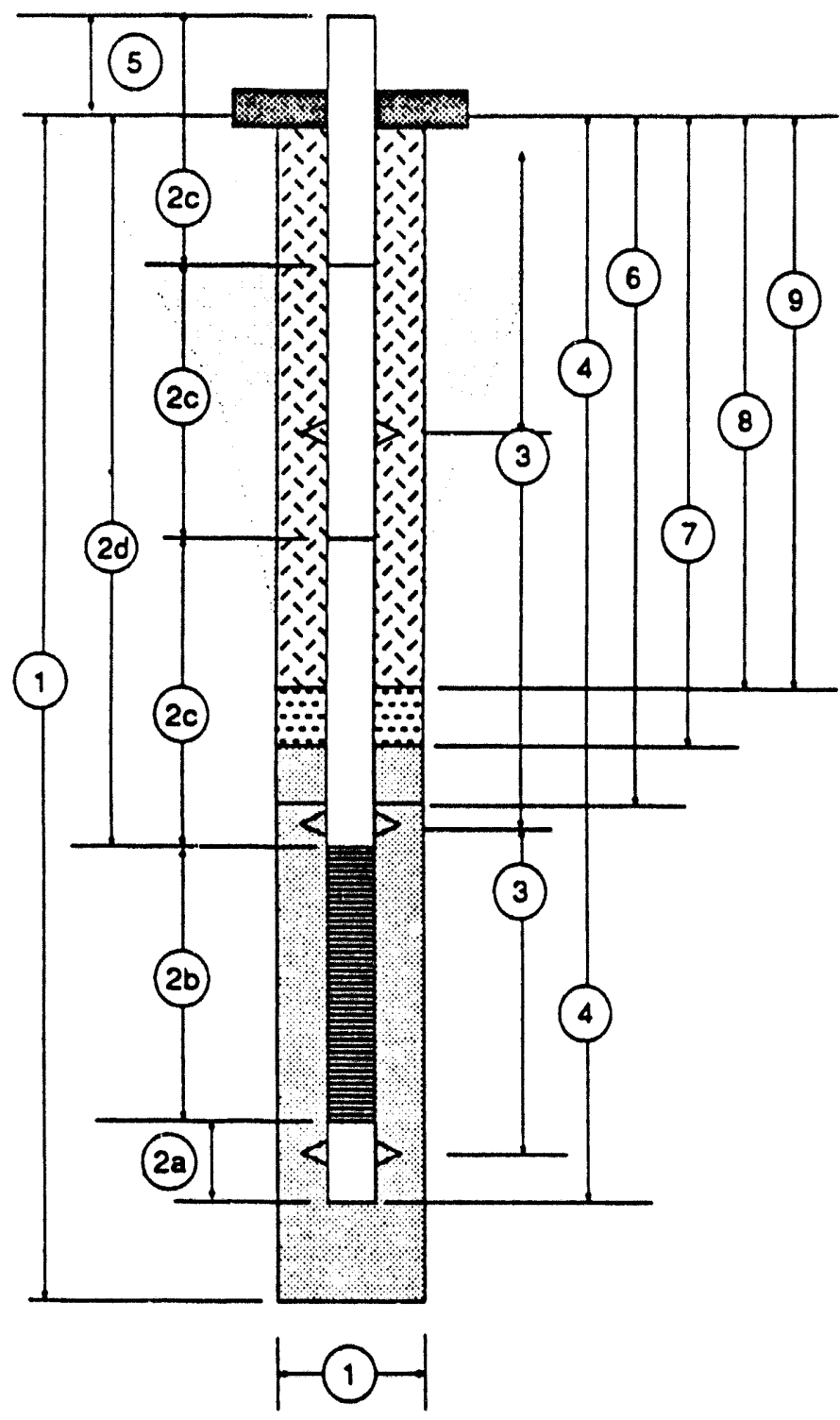

NOTE: ALL MEASUREMENTS

ARE FROM GROUND

SURFACE AT START

OF BORING - MEASUREMENTS

TO NEAREST 0.1 FOOT.

1) Tolal Drilled Depth/Hole Diameter $60.0^{\circ} / 81 / 4 "$

2) Casing/Screen Tally (Measured to Nearest 0.01 Foot)

(a) Plug Length $0.02^{\prime}$

(b) Screen Length

$4.91^{\circ}$

(c) Casing Joint Lengths (Measured in Up.

hole Sequence from Top of Screen) I) $9.89^{\circ}$

2) $9.89^{\circ}$ 3) $9.91^{\circ}$ 4) $9.92^{\circ}$ 5) $8.39^{\circ}$

(d) Depth to Top of Screen

$48.00^{\circ}$

3) Depths to Centralizers Not reguired

4) Total Depth of Installed Well

$52.9^{\circ}$

5) Casing Stick Up (Standard 2.5. A.G.S.)

6) Depth to Top of Filter Pack

$46.7^{\circ}$

7) Depth to Top of Fine Sand Seal

Not required

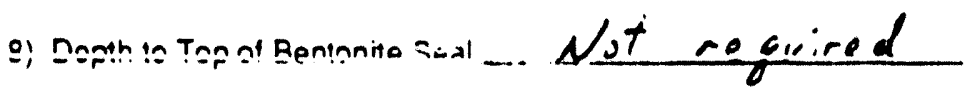

9) Thickness of Grout 2.2 


\section{MONITORING WELL CONSTRUCTION DIAGRAM}

DRILLING SUBCONTRACTOR $\leq M T C$ DRILLER Blake Cab:-

DATE OF WELL INSTALLATION $1 / 2 / \mathrm{Oa} / 92$ TECH. O.S.ICO.name Bill Joyce / IEC lPonohue

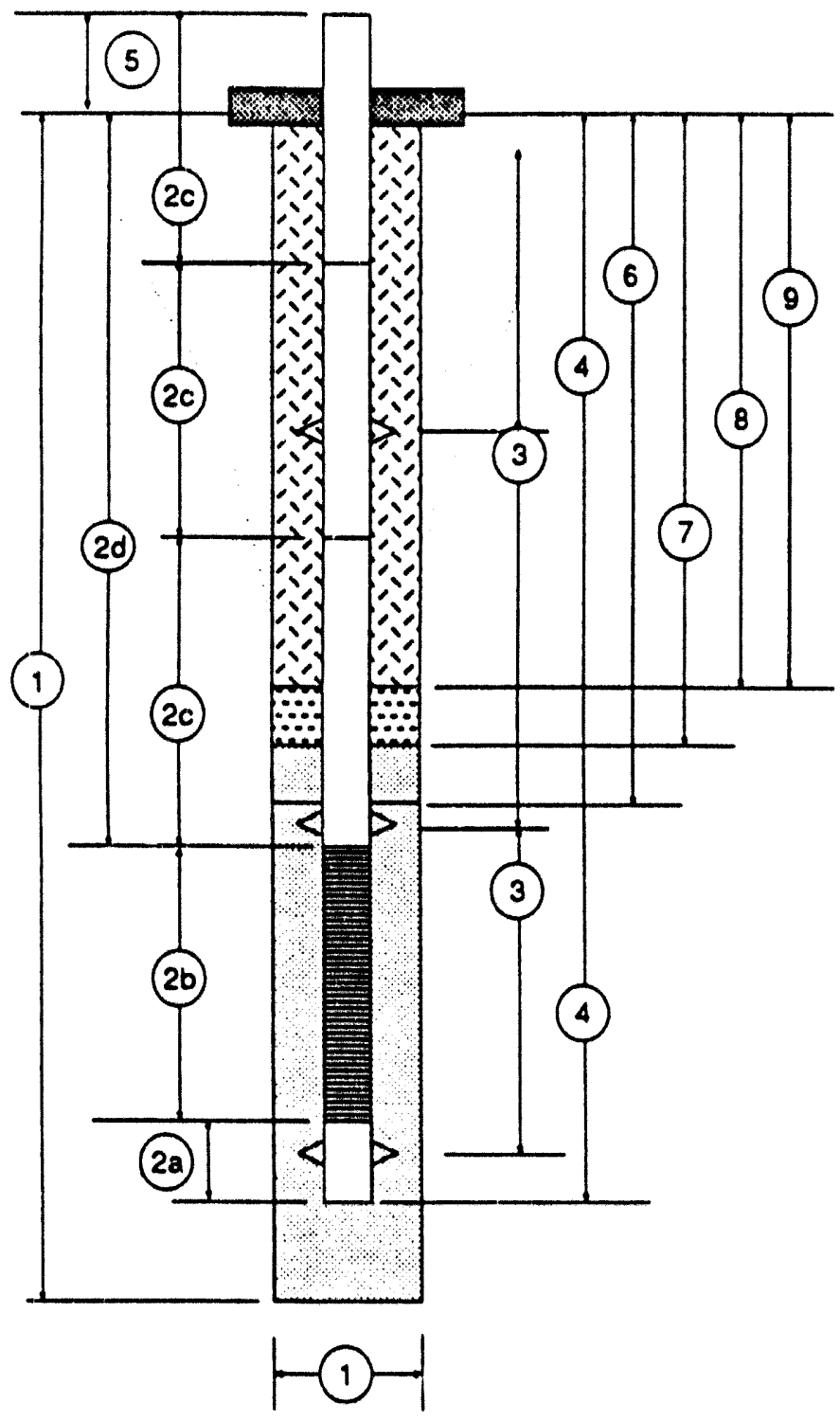

WELL NUMBER MLL'293 SRS COORDINATES

SANITARY SEAL ELEVATION
NOTE: ALL MEASUREMENTS

ARE FROM GROUND

SURFACE AT START

OF BORING - MEASUREMENTS

TO NEAREST 0.1 FOOT.

\begin{tabular}{l} 
First Gru.t \\
1) Total Drilled Depth/Hole Diameter $44.5 \%$ \\
\hline
\end{tabular}

2) Casing/Screen Tally (Measured to Nearest 0.01 Foot)
(a) Plug Longth
0.02
(b) Screen Length
$4.90^{\circ}$
(c) Casing Joint Lengths (Measured in Up- hole Sequence from Top of Screen) 1) $9.90^{\circ}$ 2) $9.89^{\circ}$ 3) $9 . 8 8 ^ { \circ } 4 \longdiv { 8 . 3 3 ^ { \circ } }$

(d) Depth to Top of Screen $38.00^{\circ}$

3) Depths to Centralizers Not required

4) Total Depth of Installed Well $42.9^{\circ}$

5) Casing Stick Up (Standard 2.5' A.G.S.) $1.6^{\circ}$

6) Depth to Top of Filler Pack 36.3

7) Depth 10 Top of Fine Sand Seal Nut ceguired

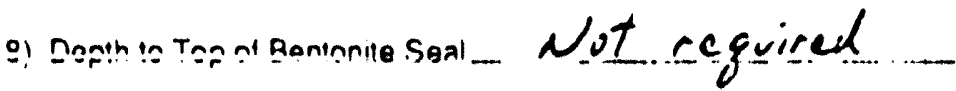

9) Thickness of Groul $2.2^{\circ}$ 
DRILLING SUBCONTRACTOR EATTE

DRILLER

$$
\text { Bloke Cab.t }
$$

DATE OF WELL INSTALLATION $12 / 03 / 92$

TeCh. O.S.ICo. name Bill Tryce/SEC Donohue

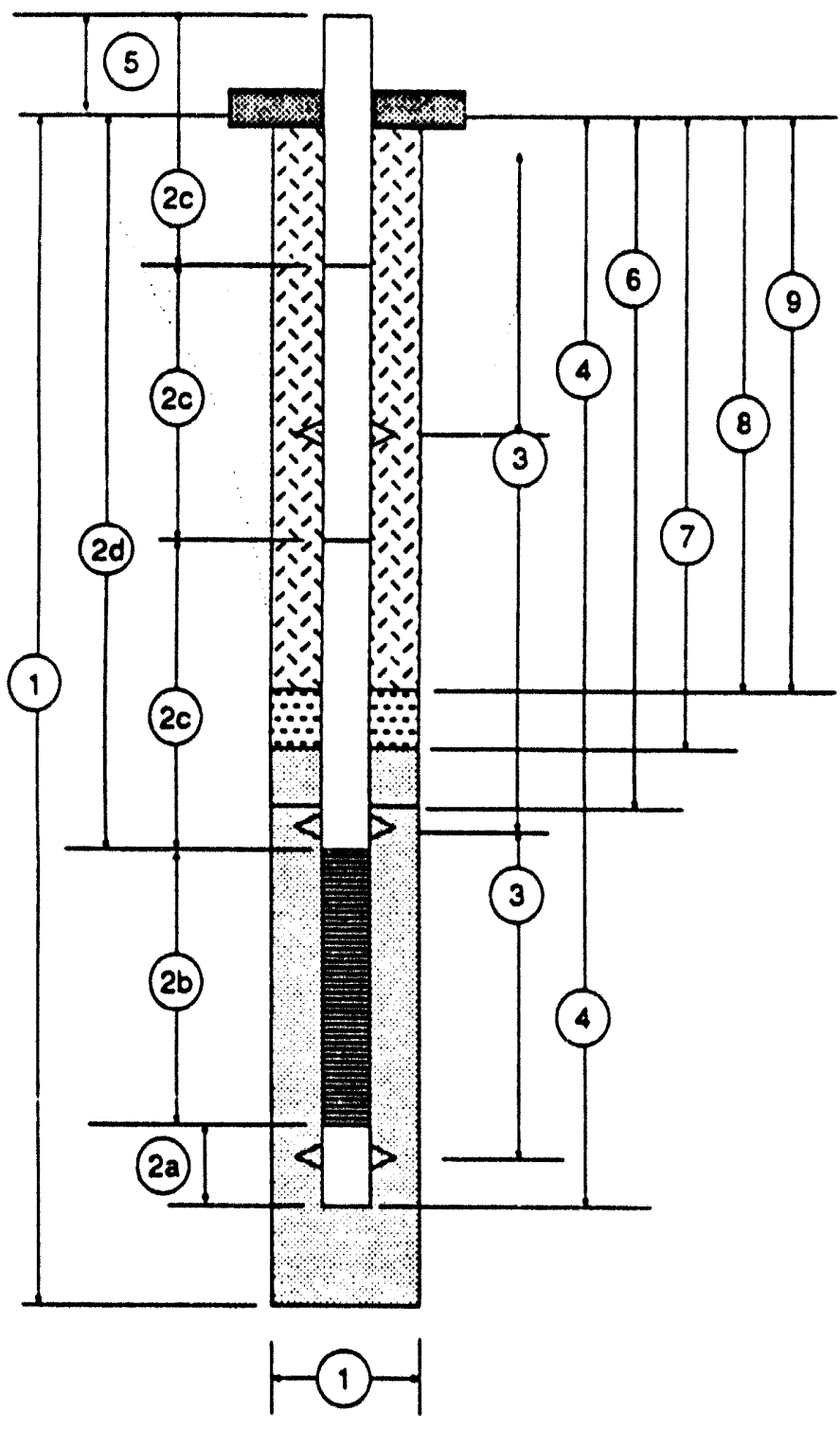

WELL NUMBER MLV $-29 C$ SRS COORDINATES

SANITARY SEAL ELEVATION
NOTE: ALL MEASUREMENTS

ARE FROM GROUND

SURFACE AT START

OF BORING - MEASUREMENTS

TO NEAREST 0.1 FOOT.

1) Total Drilled DepthHole Diameter $\begin{gathered}\text { Secued Great } \\ \text { stage } \\ 34.1 / 81 / 40\end{gathered}$

2) Casing/Sereen Tally (Measured to Nearest 0.01 Foot)

(a) Plug Length $0.04^{\prime}$

(b) Screen Length $4.89^{\circ}$

(c) Casing Joint Lengths (Measured in Uphole Sequence From Top of Srreen)

2) $9.92^{\circ} 3 / 8.16$

(d) Depth to Top of Screen

28.00

3) Dopths to Centralizers Not reguired

4) Total Depth of Installed Well

$32.9^{\circ}$

5) Casing Stick Up (Standard 2.5' A.G.S.) $1.8^{\circ}$

6) Depth to Top of Fither Pack $25.8^{\circ}$

7) Depth 10 Top of Fine Sand Seal Not regrired

Q) Danth oc Tapon Bontanito caal_Not reguired

9) Thickness ol Groul 25.8 
par 1 or 2

SEC DONOHUE

CLIENT _ WSRC

SUBUECT RF Heating Test - MHY $=29$

BY Bill Joyce DATE $12 / 03 / i 2$

CALCULATION SHEET

LOCATION

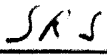

JOB NO. G.4346

Pezometer wall Construction Diagram

CHECKED BY

DATE

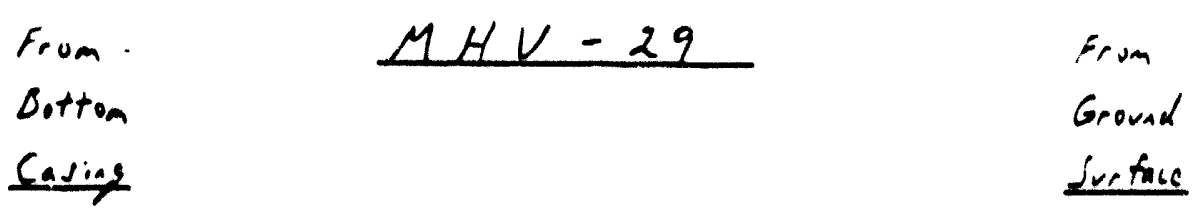

Dottom

casing

Sertace
Gravel Pack

Based on Practical

Application in the Field of

1 hay Coarse Sand $=1.2 \mathrm{ft}^{\circ}$

$\checkmark$ bag Grout $=0.6 \mathrm{ft}^{3}$

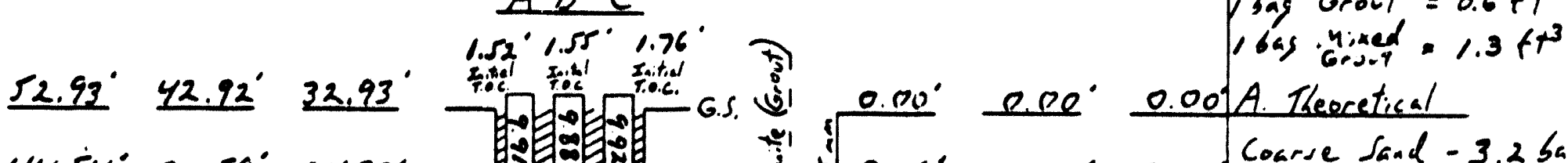

$44.54^{\circ} 34.59^{\circ} 24.27^{\circ}$

$34.62^{\circ} 24.71^{\circ} 14.85^{\circ}$

$24.71^{\circ} \quad 14.82^{\circ} \quad \frac{4.93^{\circ}}{0.04^{\circ}}$

$14.82^{\circ} \quad 4.92^{\circ}\left(0.00^{\circ}\right)$

$4.93^{\circ} \cdot \frac{0.02}{\left(0.00^{\prime}\right.}$

$\frac{0.02^{\circ}}{(0.00 \%)}$
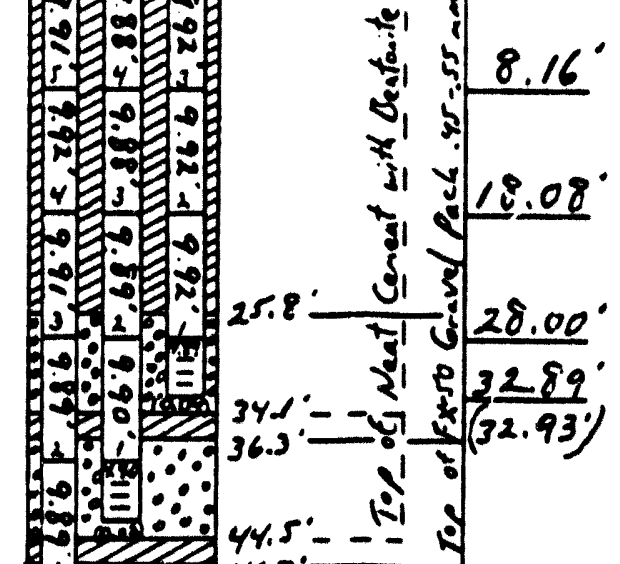

8,10000

Axy 0.000

$B=100000000$

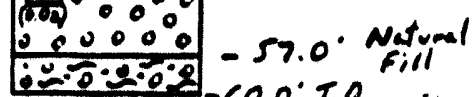

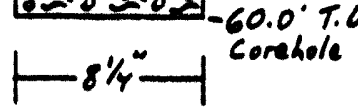

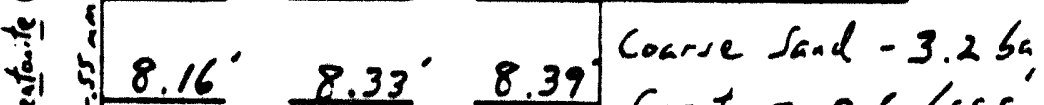

8. $8.16 \quad 8.33 \quad 8.39$

II

y11 $18.08 \quad 18.21^{\circ} \quad 18.31$

$28.10^{\circ} \quad 28.22$

$38.00^{\circ} 38.11$ $\because 90^{\circ}$

$(42.92 \%)$

48.00

52.91

$(\sqrt{2} .93)$

Coarse Saad -2.4 be,

$+11$

Grout - $0.6 \mathrm{bags}^{\prime}$ Actual

Coarse Sand - 4 bay.

Grout - 0.7 bags

B. The erefical Grout - 0.6 bugs Actual

Coarse Sand - $2.8 \mathrm{ba}$, Grout -0.7 bags

C Theoretical Coarse Sand- $2.3 \mathrm{G}$ Grout - 7.2 bugs Actual

Coarse Sand - 2.8 ba Grout - 4.7 bags 


$$
\text { PAGE } 2 \text { of } 2
$$

SEC DONOHUE

CALCULATION SHEET

CLIENT WSRC LOCATION SET JOB NO. G4346

SUBJECT RE Heating Test - MHV - 29 Pezometers Casing Tally By Bill Joyce DATE $12 / 03 / 92$ CHECKED BY DATE

$\frac{\text { Casing }}{\text { Tally }} \frac{\text { Screen }}{\text { 1) }} \frac{A .89^{\circ}}{\frac{A}{48.0^{\circ}-53.0^{\circ}}}$

2) $9.89^{\circ}$

3) $9.91^{\circ}$

4) $9.92^{\circ}$

5) $\frac{9.91^{\circ}}{49.52^{\circ}}$

- 48.00 Top of Casing

1.52 'stic heep

1) $\frac{B}{9.90^{\circ}} \quad \frac{B}{38.0^{\circ}-43.0^{\circ}}$

2) $9.89^{\circ}$

3) $9.88^{\circ}$

4) $\frac{9.88^{\circ}}{39.55^{\circ}}$

$-38.00^{\circ}$. Top of Cuing $1.55^{\circ}$ stick -up

1) $\frac{C}{9.92^{\circ}} \cdot \frac{C}{28.0^{\circ}-33.0^{\circ}}$

2) $9.92^{\circ}$

3) $\frac{9.92^{\circ}}{29.76^{\circ}}$

- 28.00 Top of Casing $1.76^{\circ}$ stick- op
Materials

List $(A B C)$

$\frac{\text { Casing }}{1 " P V C}$

Schedule yo

Tri-Loc Threads

Flush

Tinco Brand

$\frac{\text { Screen }}{\text { "PVC }}$

slotted

0.010 in. slot

Flush

Tri-Loc Threads

Times Brand

Sump

Not required

plug

Ip oe

Schedule yo

Tri-Loc Treads

Flush

Timco Brand
Screen / plug

Tally

A

\begin{tabular}{l|l|l|}
\hline \multirow{4}{*}{$4.91^{\circ}$} & & $0.10^{\circ}$ \\
\cline { 2 - 3 } & $\equiv$ & \\
& & $4.67^{\circ}$ \\
& & \\
\cline { 2 - 3 } & & $0.14^{\circ}$ \\
\hline 0.02 & plug & 0.02 \\
\hline
\end{tabular}

$B$

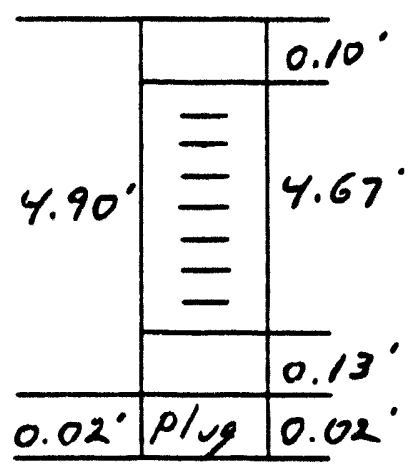

C

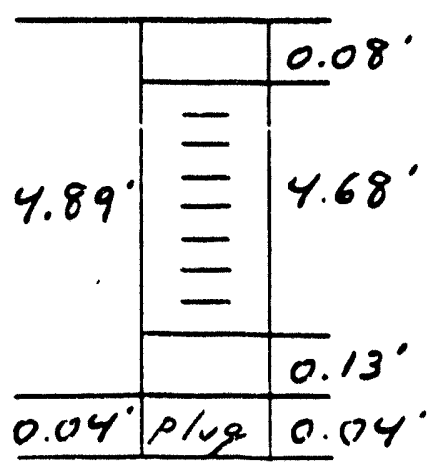


DRILLING SUBCONTRACTOR EMTC DRILLER Eirixe $C a b, t$

DATE OF WELL INSTALLATION $12 / \mathrm{CZ} / 92$ TECH. O.S.ICO.NAME Dill Joyce/SEC Monohue

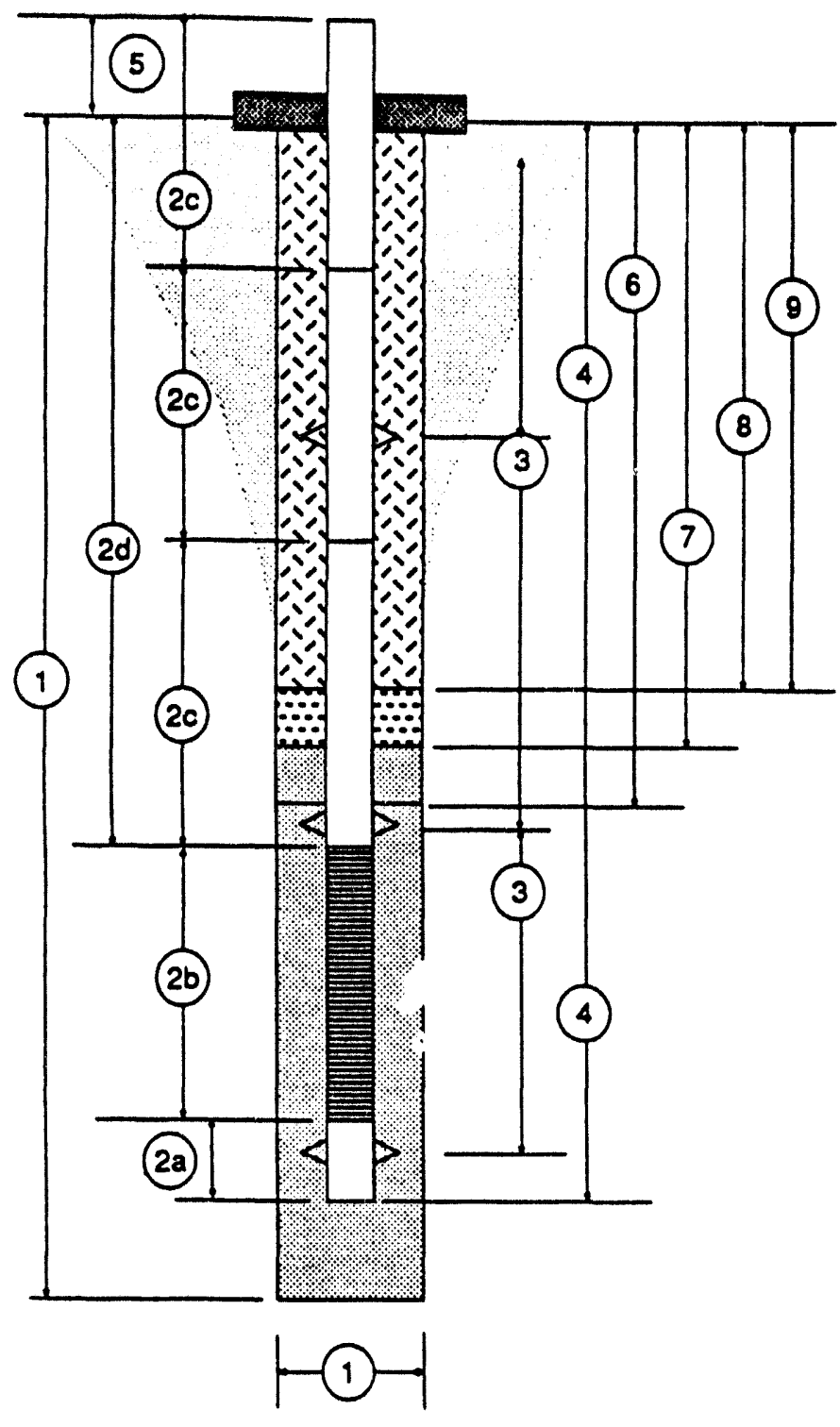

WELL NUMBER $M \angle V-20 T$ SRS COORDINATES SANITARY SEAL ELEVATION NOTE: ALL MEASUREMENTS ARE FROM GROUND SUAFACE AT START OF BORING - MEASUREMENTS TO NEAREST 0.1 FOOT.

Fibor Optics Temperature Seasors 1) Total Drilled Depth/Hole Diameter $57.0^{\circ} / 81 / 4$ "

2) Casing/Screen Tally (Measured to Nearest 0.01 Foot)

(a) Sump \& Plug Longth _.Not required

(b) Screen Length Not reguired (c) Casing Joint Lengths (Measured in Up
hole Sequenco From Top of Scroen) $10.16^{\circ}$
with threads $2 / 10.02^{\circ} 3 / 10.03^{\circ}$
$\begin{array}{ll}\text { 4) } 10.02^{\circ} & \text { s) } 9.77^{\circ}\end{array}$ 4) $10.02^{\circ}$ 5) $9.72^{\circ}$

(d) Depth to Iepolforoen $28.00^{\circ}, 38.00^{\circ}, 48.00^{\circ}$

3) Depths to Centralizers Not required

4) Total Depth of Installed Well 50. $0^{\prime}$

5) Casing Stick Up (Standard 2.5 A.G.S.) _ $2.3^{\circ}$

6) Depth to Top of Filter Pack $8.5^{\circ}$

7) Depth to Top of Fine Sand Seal Nut reguired

8) Depth to Top of Bentonite S:al Net required

9) Thickness of Grout $8 . \mathrm{r}^{\prime}$ 


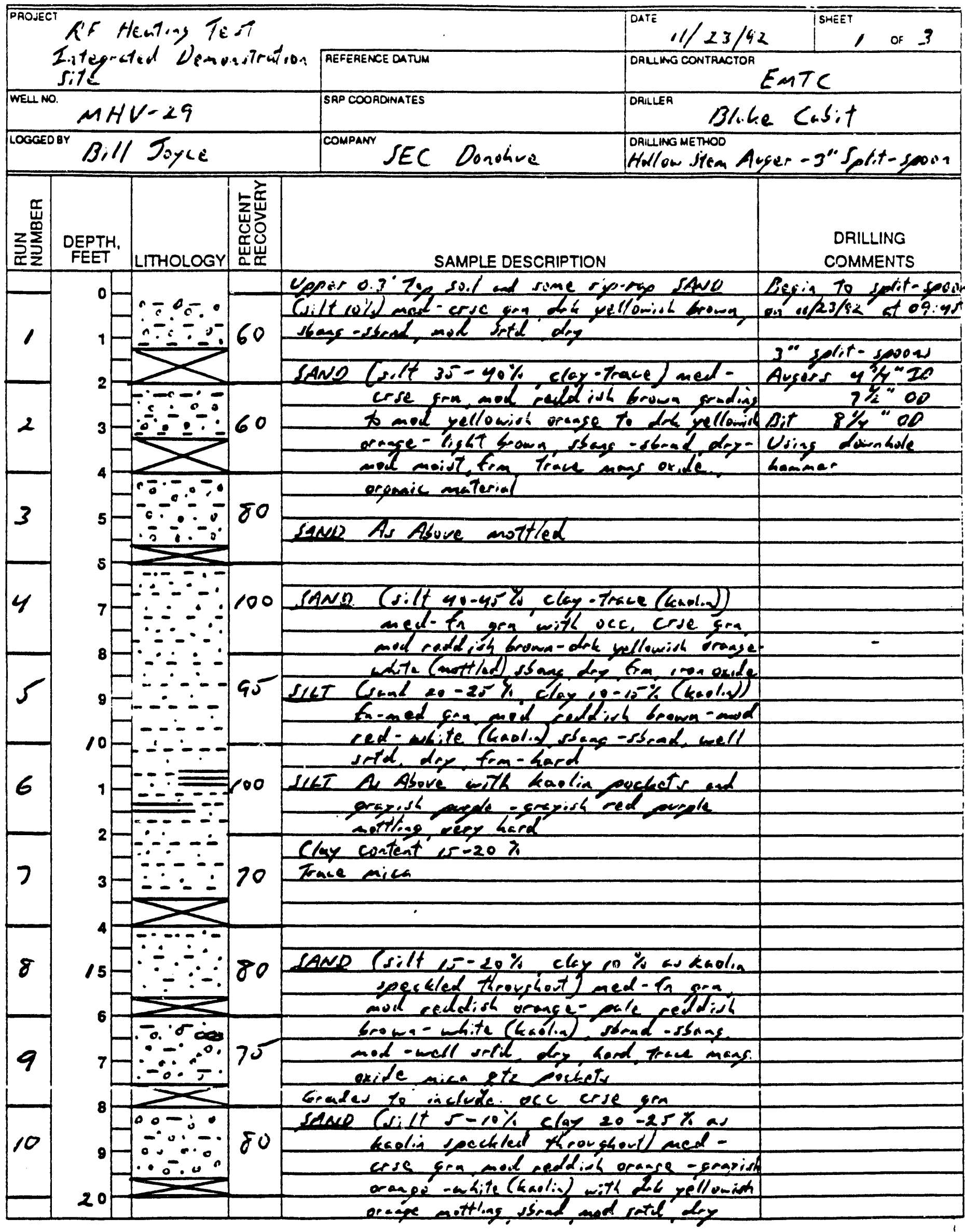


OSR 30.3

FIELD GEOLOGIC LOG

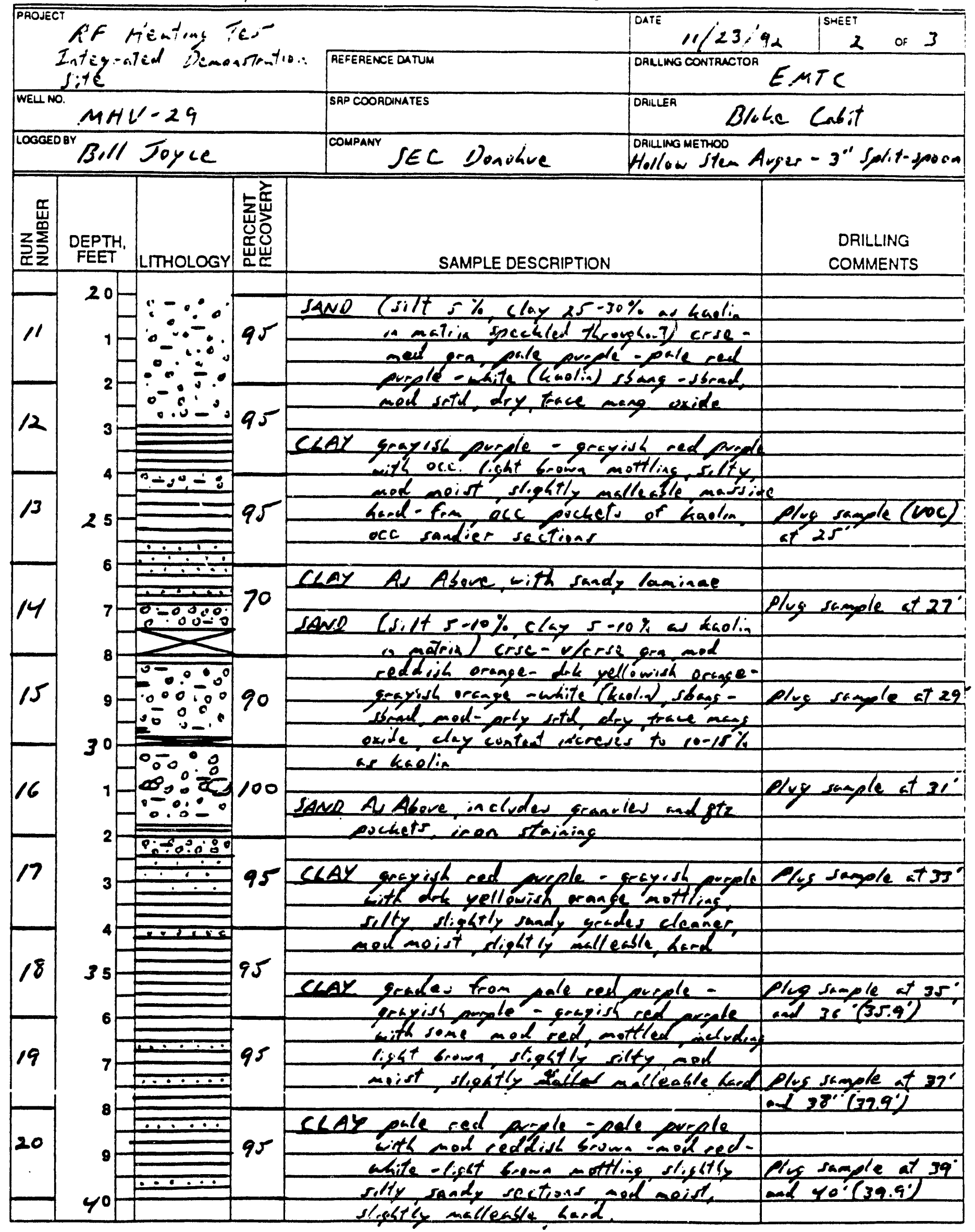


OSR so-s

FIELD GEOLOGIC LOG

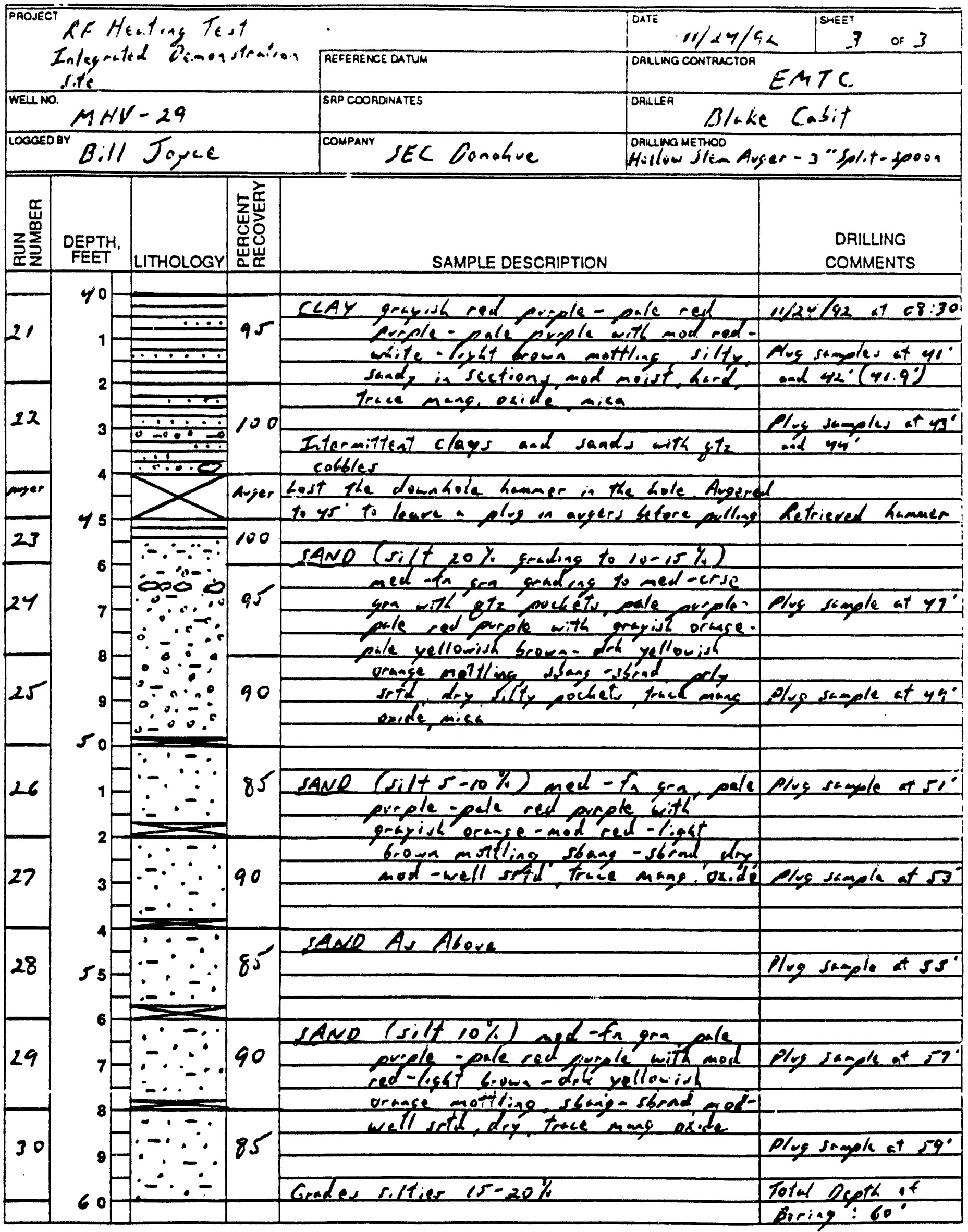


ORILING SUBCONTRACTOR Graves

DRILER $B$. Cunningham

DATE OF WELL INSTALATON $05 / 13 / 92$

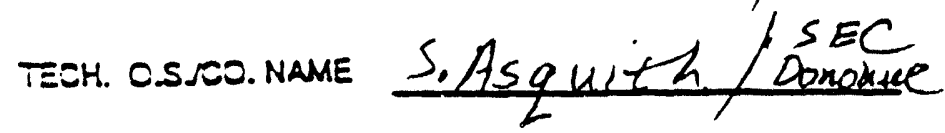

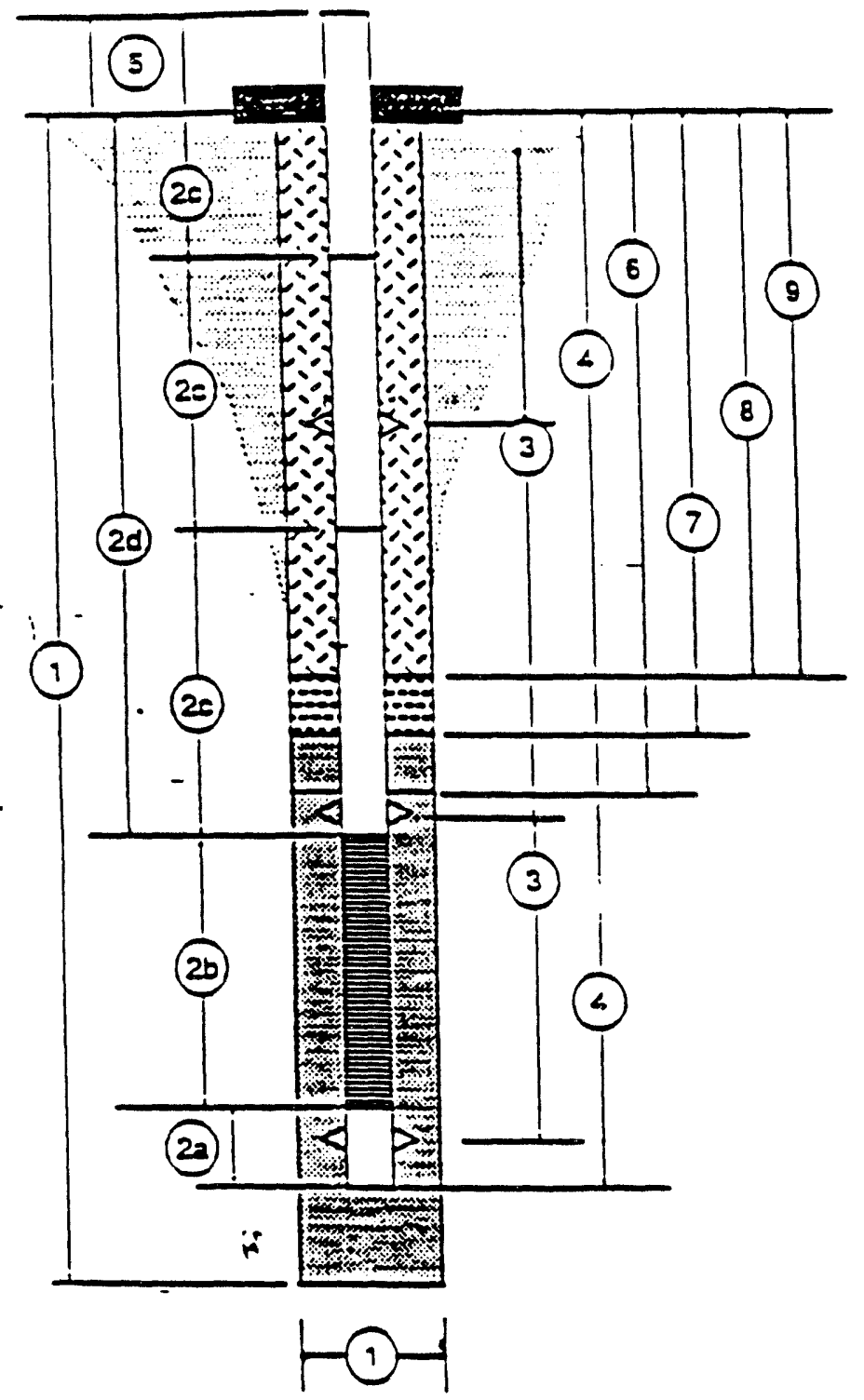

Below screen

Filter Pack: 149.2 to 162.8

Bentonite Seal: 162.8 to 167.7

Fx 50 sand: 167.7 to 210.0
WELL NUMBER AHTT- $16 C$

SRS COORDINATES $102,430,85 \mathrm{~N}, 48,672,81 \mathrm{E}$

SANTARY SEAL EIEVATION

LOUDLLVEPPE

NOTE: ALL MEASUREMENTS

AAE FAOM GAOUND

OF BORING - MEASUREMENTS

TO NEAREST 0.1 FOOT.

1) Total Driled Deothntole Diamearer 210.0'/12".

2) Casmorsecoen Tally (Measured wo Noaresr 0.01 Foer)

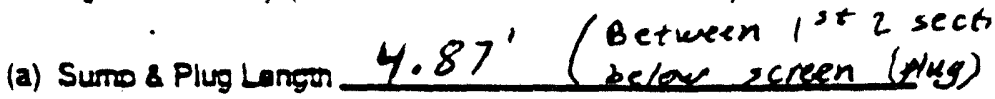

(b) Screen Langen 4,83

- -

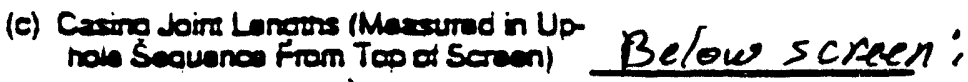
$0.46^{\prime}$ (plug), $19.77^{\prime}, 19.77^{\prime}, 4.87^{\prime}$ Above screen: 10.03', $19.74^{\prime}$ $\frac{19.75^{\prime}, 20.24^{\prime}, 19.75^{\prime}, 19.74^{\prime},}{20.24^{\prime}, 20.244^{\prime}, 6.77^{\prime}}$

(a) Dean wo Tap of Scroon $154,0^{\prime}$

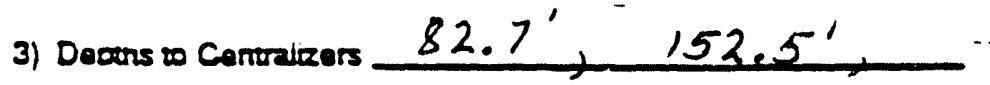
$160.3^{\prime}, 202.2^{\prime}$

4) Tearal Dexen of instailod Woll $203.7^{\prime}$

5) Casing Stiox Up (Stancerd 25 AG.S.) $2.5^{\prime}$

ต) Dapen wo Too of Fithor Pax 149.2

$\pi$ Deoth wo Too of Fine Sard Sal $147,5^{\prime}$

8) Deon wo Too ol Bemenne Seal_ $137,4^{\prime}$

Depths to Tor of Tomegraphy Probes:

1) Thioxness of Grour

$137.0^{\prime}$

(Depths in parenthosies were measured when installed on casing.

All other depths are calculated from measured interva/s between cable splices) $(200.0), 186.7,173.4,160.1 /(159.8), 146.8,133.5,120.2,106.9,93.6$, $80.3,67.0,53.7,40.4,27.1 .12 .8$ ก.w (A.w) 
FIELD GEOLOGIC LOG

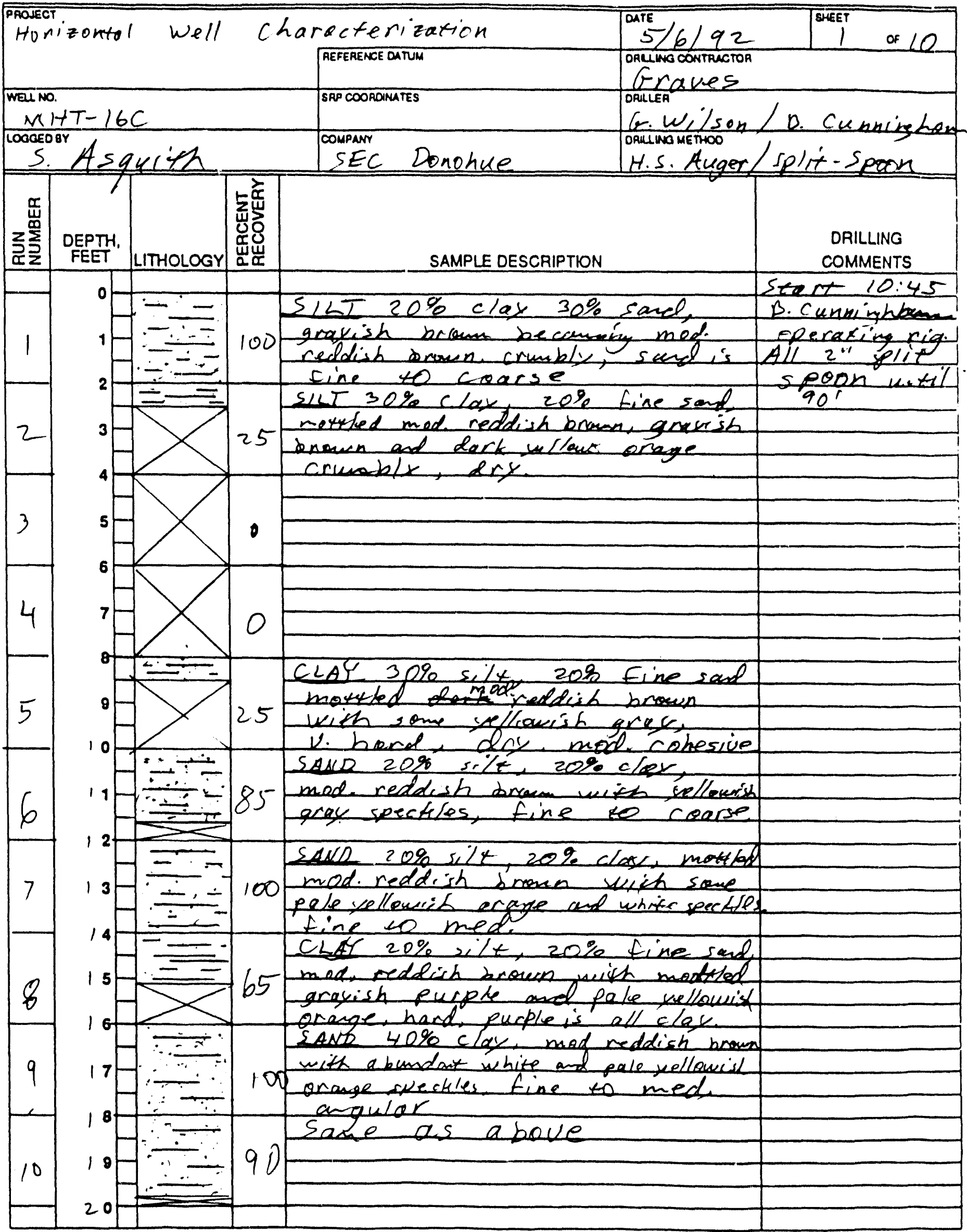




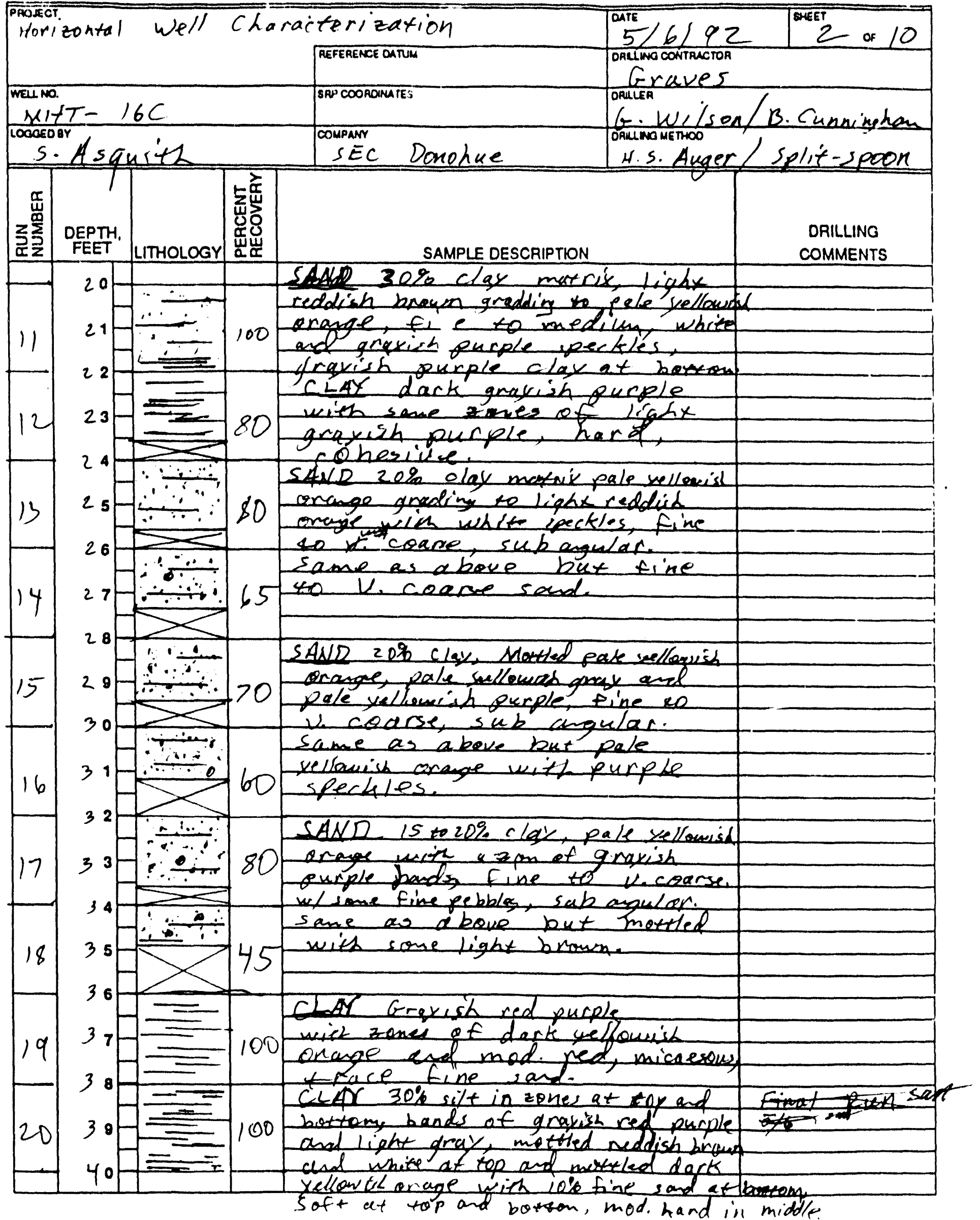


FIELD GEOLOGIC LOG

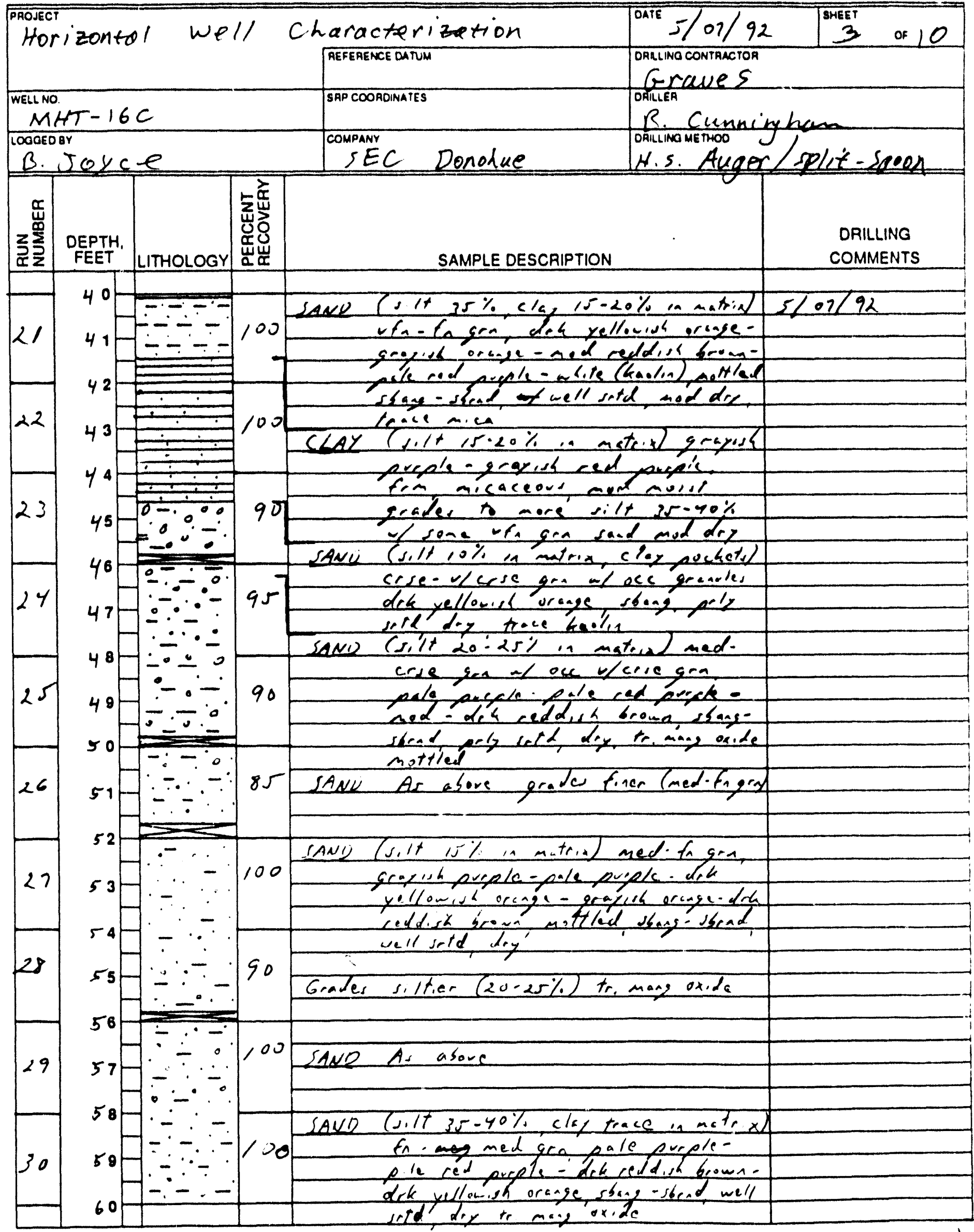


OSR $30 \cdot 3$

FIELD GEOLOGIC LOG

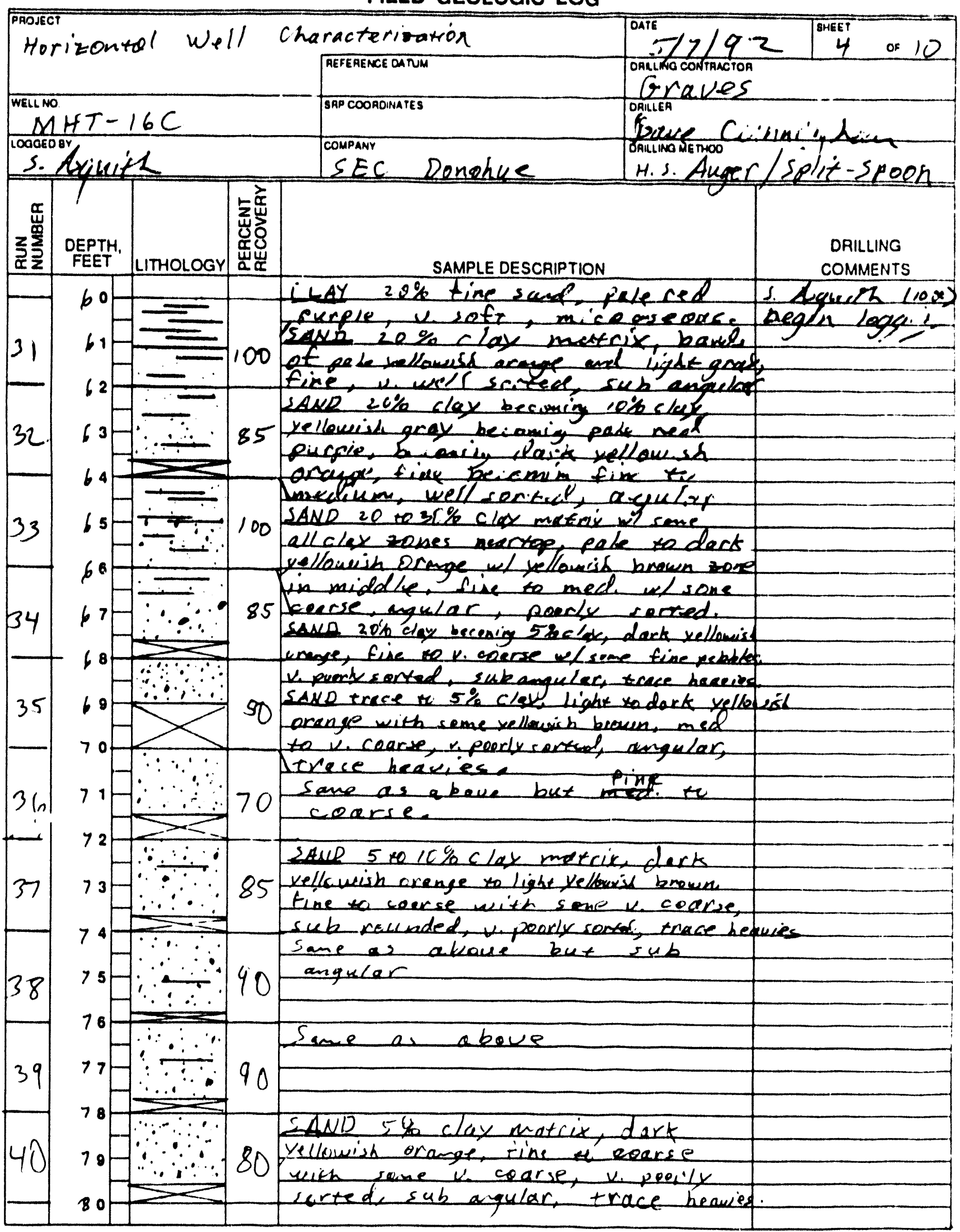


FIELD GEOLOGIC LOG

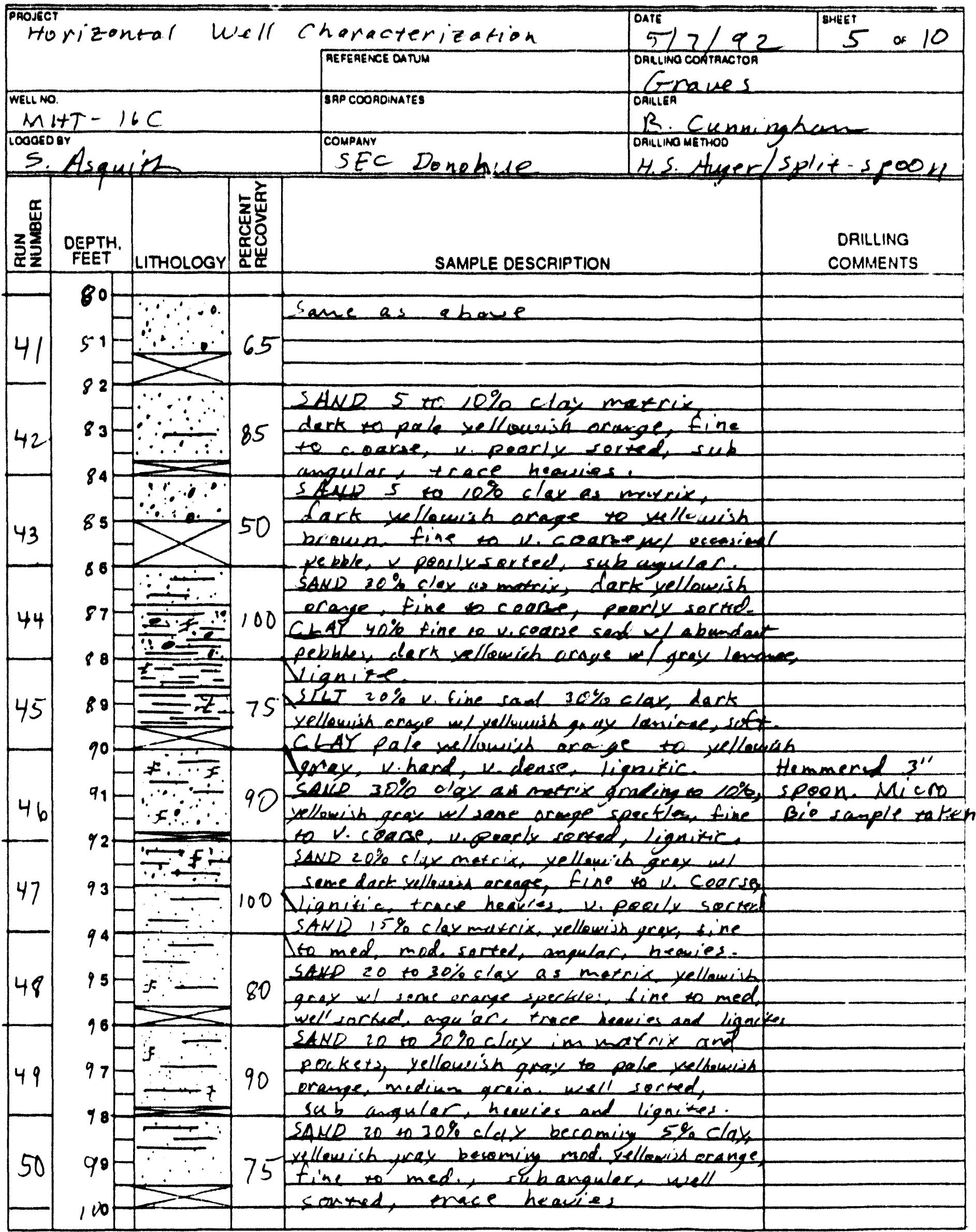




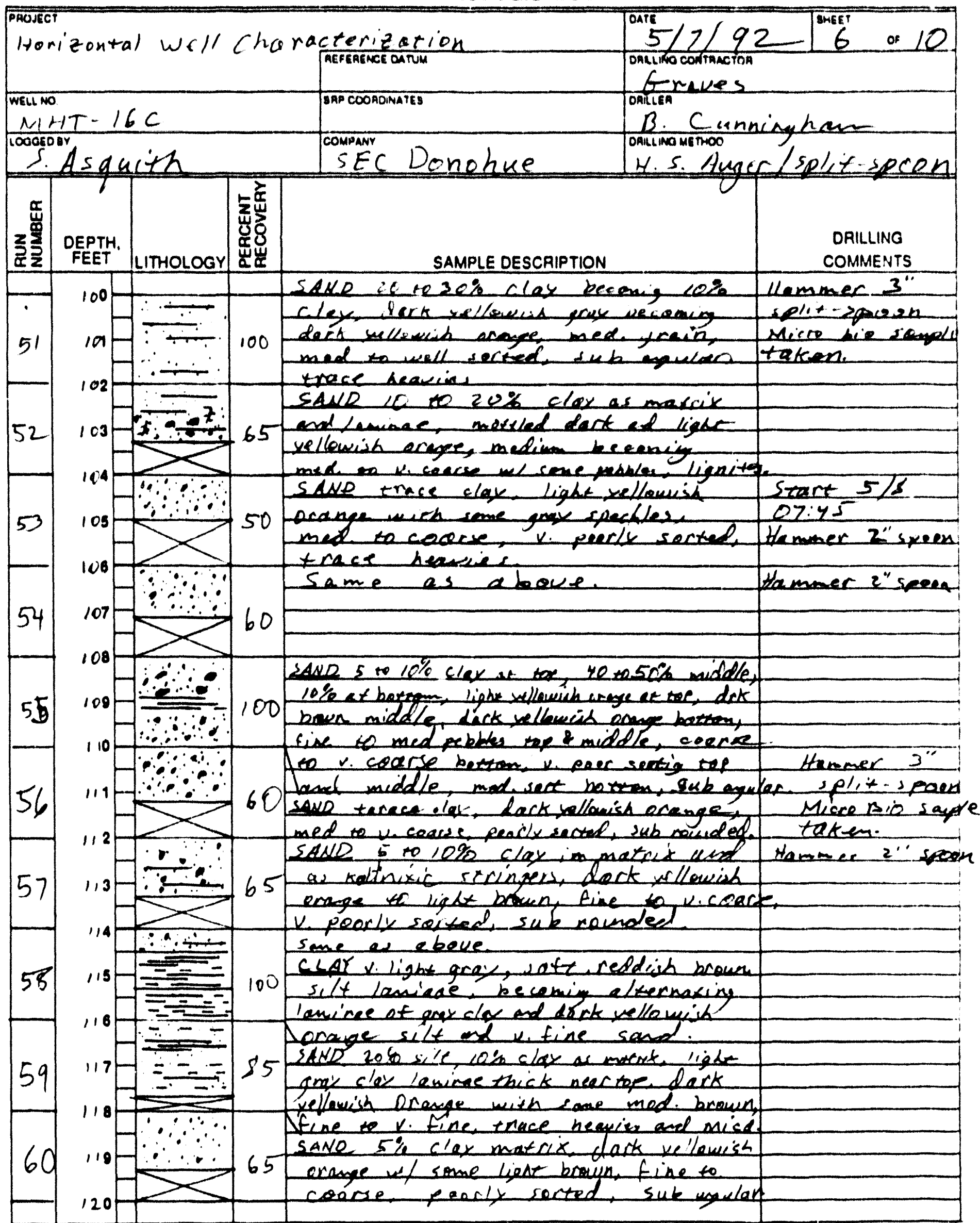




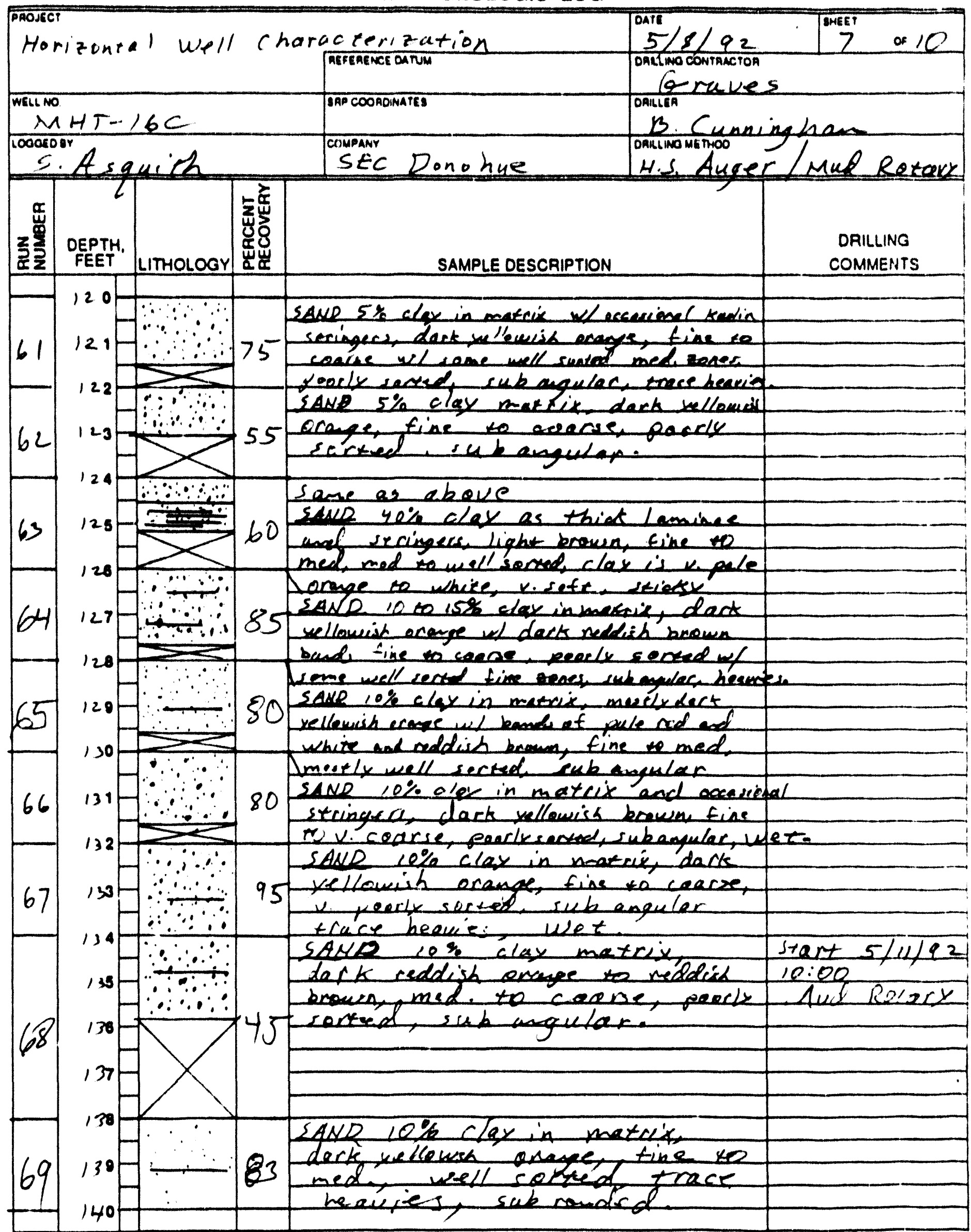




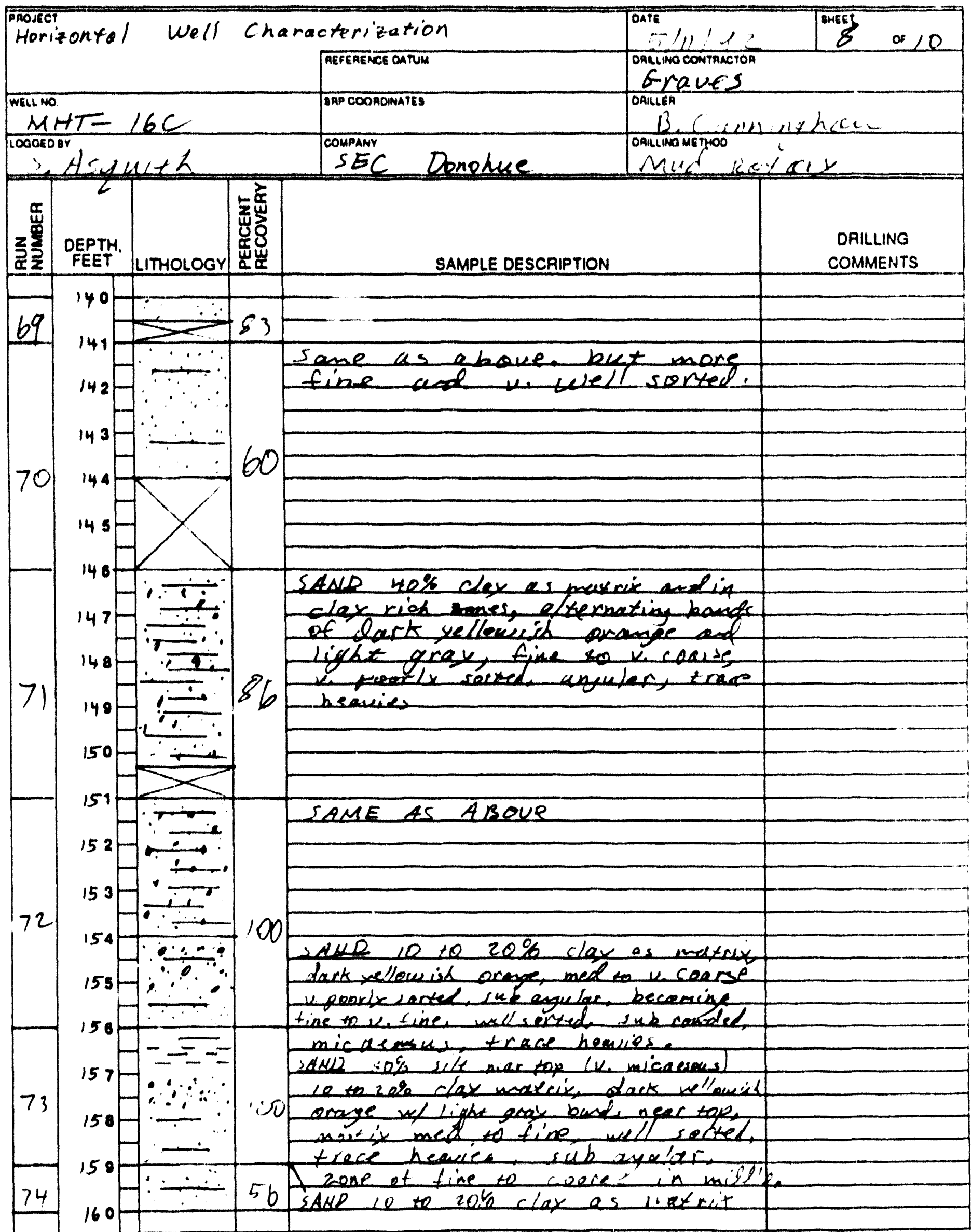









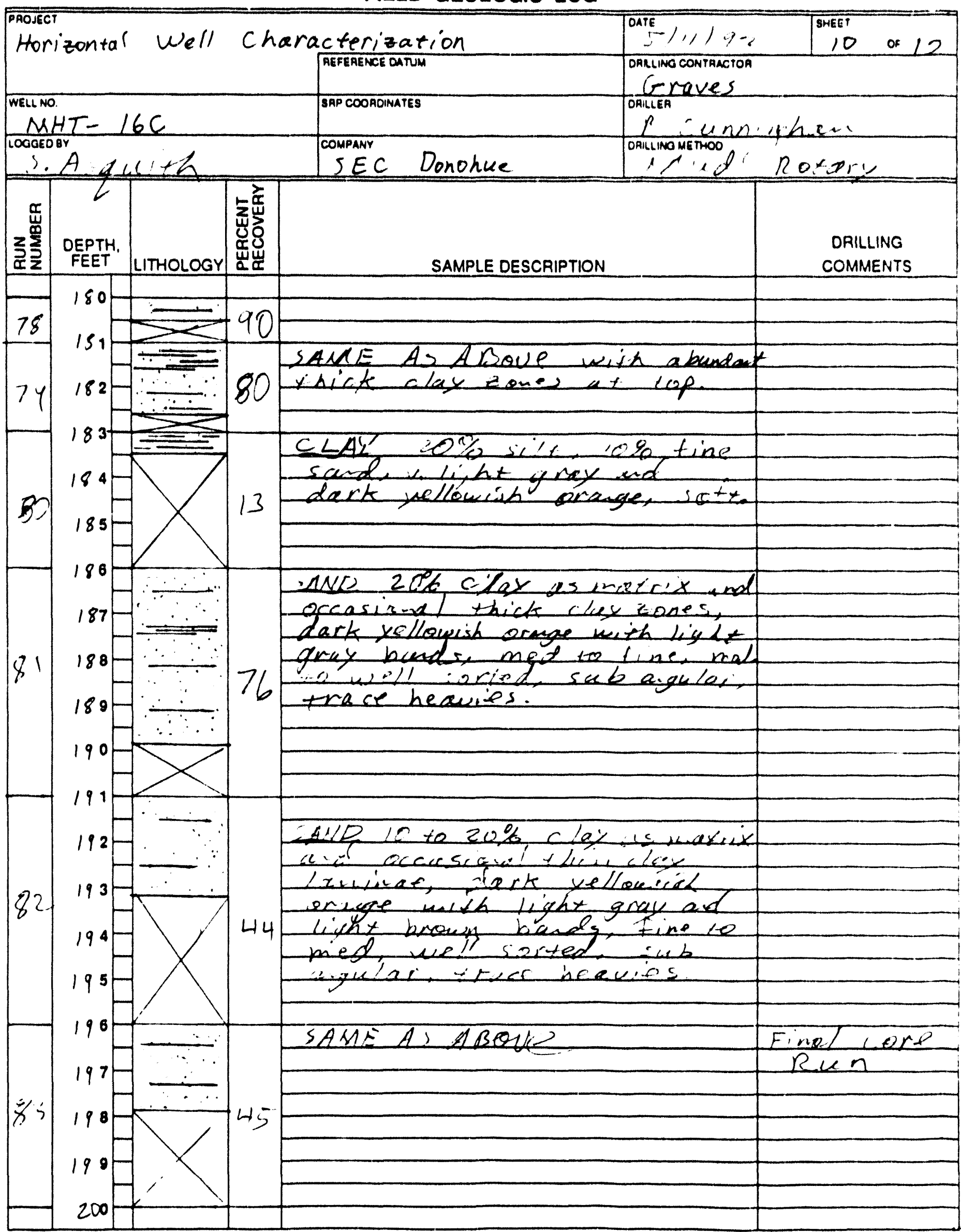




\section{Appendix II-Moisture Content or Soil Water from Six Sediment Cores}


Init $\quad n$

I.D. Date (feet) Gravel Sand Clay

\begin{tabular}{|c|c|c|c|}
\hline MHV 21 & $11 / 12$ & 25 & 1 \\
\hline MHV 21 & $11 / 12$ & 25 & 1 \\
\hline MHV 21 & $11 / 12$ & 27 & 5 \\
\hline MHV 21 & $11 / 12$ & 27 & 5 \\
\hline MHV 21 & $11 / 12$ & 29 & 10 \\
\hline MHV 21 & $11 / 12$ & 29 & 10 \\
\hline MHV 21 & $11 / 12$ & 31 & 5 \\
\hline MHV 21 & $11 / 12$ & 31 & 5 \\
\hline MHV 21 & $11 / 12$ & 33 & 5 \\
\hline MHV 21 & $11 / 12$ & 33 & 5 \\
\hline MHV 21 & $11 / 12$ & 35 & 0 \\
\hline MHV 21 & $11 / 12$ & 35 & 0 \\
\hline MHV 21 & $11 / 12$ & 36 & 0 \\
\hline MHV 21 & $11 / 12$ & 36 & 0 \\
\hline MHV 21 & $11 / 13$ & 37 & 0 \\
\hline MHV 21 & $11 / 13$ & 37 & 0 \\
\hline MHV 21 & $11 / 13$ & 38 & 0 \\
\hline MHV 21 & $11 / 13$ & 38 & 0 \\
\hline MHV 21 & $11 / 13$ & 39 & 0 \\
\hline MHV 21 & $11 / 13$ & 39 & 0 \\
\hline MHV 21 & $11 / 13$ & 40 & 0 \\
\hline MHV 21 & $11 / 13$ & 40 & 0 \\
\hline MHV 21 & $11 / 13$ & 41 & 0 \\
\hline MHV 21 & $11 / 13$ & 41 & 0 \\
\hline MHV 21 & $11 / 13$ & 42 & 0 \\
\hline MHV 21 & $11 / 13$ & 42 & 0 \\
\hline MHV 21 & $11 / 13$ & 43 & 0 \\
\hline MHV 21 & $11 / 13$ & 43 & 0 \\
\hline MHV 21 & $11 / 13$ & 44 & 0 \\
\hline MHV 21 & $11 / 13$ & 44 & 0 \\
\hline MHV 21 & $11 / 13$ & 45 & 3 \\
\hline MHV 21 & $11 / 13$ & 45 & 3 \\
\hline MHV 21 & $11 / 13$ & 47 & 0.1 \\
\hline MHV 21 & $11 / 13$ & 47 & 0.1 \\
\hline MHV 21 & $11 / 13$ & 49 & 0 \\
\hline MHV 21 & $11 / 13$ & 49 & 0 \\
\hline MHV 21 & $11 / 13$ & 51 & 0 \\
\hline
\end{tabular}

FINAL

t1 2

dry Initial vial

$\%$

$20 \mathrm{SD}$

20 SD

15 SD

$15 \mathrm{SD}$

20 SD

$20 \mathrm{SD}$

20 SD

20 SD

$15 \mathrm{SD}$

15 SD

$80 \mathrm{CL}$

$80 \mathrm{CL}$

65 SDCL

$65 \mathrm{SDCL}$

\section{$65 \mathrm{SDCL}$}

$65 \mathrm{SDCL}$

70 SDCL

$70 \mathrm{SDCL}$

$80 \mathrm{CL}$

$80 \mathrm{CL}$

$80 \mathrm{CL}$

$80 \mathrm{CL}$

$85 \mathrm{CL}$

$85 \mathrm{CL}$

$85 \mathrm{CL}$

$85 \mathrm{CL}$

$90 \mathrm{CL}$

$90 \mathrm{CL}$

$90 \mathrm{CL}$

$90 \mathrm{CL}$

57 SDCL

$57 \mathrm{SDCL}$

25 CLSD

25 CLSD

20 SD

20 SD

10 SD

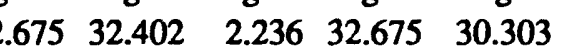
$\begin{array}{llllll}33.591 & 33.142 & 3.059 & 33.591 & 30.308\end{array}$ $\begin{array}{llllll}34.922 & 33.785 & 4.122 & 34.922 & 30.232\end{array}$ $\begin{array}{llllll}36.013 & 34.649 & 4.938 & 36.013 & 30.393\end{array}$ $\begin{array}{llllll}36.054 & 35.511 & 5.308 & 36.054 & 30.475\end{array}$ $\begin{array}{llllll}36.507 & 35.802 & 5.805 & 36.507 & 30.350\end{array}$ $\begin{array}{llllll}35.874 & 35.332 & 5.159 & 35.874 & 30.444\end{array}$ $\begin{array}{llllll}35.247 & 34.792 & 4.479 & 35.247 & 30.541\end{array}$ $\begin{array}{llllll}34.496 & 34.095 & 4.008 & 34.496 & 30.288\end{array}$ $\begin{array}{llllll}36.694 & 35.979 & 6.050 & 36.694 & 30.287\end{array}$ $\begin{array}{llllll}36.074 & 34.824 & 5.065 & 36.074 & 30.384\end{array}$ $\begin{array}{llllll}36.473 & 35.183 & 5.462 & 36.473 & 30.366\end{array}$ $\begin{array}{llllll}36.695 & 35.414 & 5.783 & 36.695 & 30.272\end{array}$ $\begin{array}{lllll}35.801 & 34.733 & 5.046 & 35.801 & 30.221\end{array}$ $\begin{array}{llllll}38.769 & 37.258 & 7.442 & 38.769 & 30.572\end{array}$ $\begin{array}{llllll}37.267 & 36.013 & 6.133 & 37.267 & 30.507\end{array}$ $\begin{array}{llllll}36.081 & 34.842 & 5.013 & 36.081 & 30.449\end{array}$ $\begin{array}{llllll}36.805 & 35.387 & 5.674 & 36.805 & 30.422\end{array}$ $\begin{array}{llllll}37.137 & 35.713 & 5.709 & 37.137 & 30.716\end{array}$ $\begin{array}{llllll}35.221 & 34.052 & 4.284 & 35.221 & 30.353\end{array}$ $\begin{array}{llllll}37.043 & 35.626 & 5.981 & 37.043 & 30.354\end{array}$ $\begin{array}{llllll}38.392 & 36.845 & 7.262 & 38.392 & 30.357\end{array}$ $\begin{array}{llllll}37.535 & 36.278 & 6.376 & 37.535 & 30.531\end{array}$ $\begin{array}{llllll}37.200 & 35.963 & 6.205 & 37.200 & 30.377\end{array}$ $\begin{array}{llllll}36.494 & 35.487 & 5.696 & 36.494 & 30.295\end{array}$ $\begin{array}{llllll}36.011 & 34.784 & 5.075 & 36.011 & 30.323\end{array}$ $\begin{array}{llllll}38.177 & 36.698 & 6.887 & 38.177 & 30.551\end{array}$ $\begin{array}{llllll}35.884 & 34.713 & 4.906 & 35.884 & 30.393\end{array}$ $\begin{array}{llllll}35.629 & 34.511 & 5.001 & 35.629 & 30.069\end{array}$ $\begin{array}{llllll}36.948 & 35.634 & 6.051 & 36.948 & 30.240\end{array}$ $\begin{array}{llllll}36.558 & 35.476 & 5.768 & 36.558 & 30.249\end{array}$ $\begin{array}{llllll}36.425 & 35.843 & 5.661 & 36.425 & 30.473\end{array}$ $\begin{array}{llllll}36.055 & 35.448 & 5.347 & 36.055 & 30.405\end{array}$ $\begin{array}{llllll}36.975 & 36.270 & 6.288 & 36.975 & 30.335\end{array}$ $\begin{array}{llllll}37.688 & 36.905 & 6.409 & 37.688 & 30.888\end{array}$ $\begin{array}{lllll}36.427 & 35.884 & 5.818 & 36.427 & 30.338\end{array}$ $\begin{array}{llllll}36.927 & 35.454 & 5.906 & 36.927 & 30.285\end{array}$ moist Sat $\begin{array}{lrll}\text { g } & \text { wgt } & & \text { cc } \\ 2.37 & 6 & 0.376 & 1.36\end{array}$

$\begin{array}{llll}3.28 & 7 & 0.376 & 1.86\end{array}$

$\begin{array}{llll}4.69 & 12 & 0.370 & 2.47\end{array}$

$\begin{array}{llll}5.62 & 12 & 0.370 & 2.96\end{array}$

$\begin{array}{llll}5.58 & 5 & 0.376 & 3.22\end{array}$

$\begin{array}{llll}6.16 & 6 & 0.376 & 3.52\end{array}$

$\begin{array}{llll}5.43 & 5 & 0.376 & 3.13\end{array}$

$\begin{array}{llll}4.71 & 5 & 0.376 & 2.72\end{array}$

$\begin{array}{llll}4.21 & 5 & 0.370 & 2.41\end{array}$

$\begin{array}{llll}6.41 & 6 & 0.370 & 3.63\end{array}$

$\begin{array}{llll}5.69 & 11 & 0.454 & 3.55\end{array}$

$\begin{array}{llll}6.11 & 11 & 0.454 & 3.83\end{array}$

$\begin{array}{llll}6.42 & 10 & 0.435 & 3.91\end{array}$

$\begin{array}{llll}5.58 & 10 & 0.435 & 3.41\end{array}$

$\begin{array}{llll}8.20 & 9 & 0.435 & 5.03\end{array}$

$\begin{array}{llll}6.76 & 9 & 0.435 & 4.14\end{array}$

$\begin{array}{llll}5.63 & 11 & 0.441 & 3.43\end{array}$

$\begin{array}{llll}6.38 & 11 & 0.441 & 3.88\end{array}$

$\begin{array}{llll}6.42 & 11 & 0.454 & 4.01\end{array}$

$\begin{array}{lllll}6.64 & 11 & 0.454 & 4.14\end{array}$

$\begin{array}{llll}4.87 & 12 & 0.454 & 3.01\end{array}$

$\begin{array}{llll}6.69 & 11 & 0.454 & 4.20\end{array}$

$\begin{array}{llll}8.04 & 10 & 0.461 & 5.16\end{array}$

$\begin{array}{llll}7.00 & 9 & 0.461 & 4.53\end{array}$

$\begin{array}{llll}6.82 & 9 & 0.461 & 4.41\end{array}$

$\begin{array}{llll}6.20 & 8 & 0.461 & 4.05\end{array}$

$\begin{array}{llll}5.69 & 11 & 0.467 & 3.65\end{array}$

$\begin{array}{lllll}7.63 & 10 & 0.467 & 4.96\end{array}$

$\begin{array}{llll}5.49 & 11 & 0.467 & 3.53\end{array}$

$\begin{array}{llll}5.56 & 10 & 0.467 & 3.60\end{array}$

$\begin{array}{lllll}6.71 & 10 & 0.424 & 4.01\end{array}$

$\begin{array}{llll}6.31 & 9 & 0.424 & 3.82\end{array}$

$\begin{array}{llll}5.95 & 5 & 0.383 & 3.47\end{array}$

$\begin{array}{llll}5.65 & 5 & 0.383 & 3.28\end{array}$

$\begin{array}{llll}6.64 & 5 & 0.376 & 3.82\end{array}$

$\begin{array}{llll}6.80 & 6 & 0.376 & 3.89\end{array}$

$\begin{array}{llll}6.09 & 4 & 0.363 & 3.45\end{array}$
S

Q $>75 \%<75$

sand\& sand\&

sat. Vw/Vt gravel gravel $\%$

\begin{tabular}{|c|c|c|}
\hline 27 & 0.10 & 27 \\
\hline 32 & 0.12 & 32 \\
\hline 62 & 0.23 & 62 \\
\hline 62 & 0.23 & 62 \\
\hline 22 & 0.08 & 22 \\
\hline 27 & 0.10 & 27 \\
\hline 23 & 0.09 & 23 \\
\hline 22 & 0.08 & 22 \\
\hline 23 & 0.08 & 23 \\
\hline 27 & 0.10 & 27 \\
\hline 39 & 0.18 & NS \\
\hline 37 & 0.17 & NS \\
\hline 38 & 0.16 & NS \\
\hline 36 & 0.16 & NS \\
\hline 35 & 0.15 & NS \\
\hline 35 & 0.15 & NS \\
\hline 41 & 0.18 & NS \\
\hline 41 & 0.18 & NS \\
\hline 39 & 0.18 & NS \\
\hline 39 & 0.18 & NS \\
\hline 43 & 0.19 & NS \\
\hline 37 & 0.17 & NS \\
\hline 33 & 0.15 & NS \\
\hline 30 & 0.14 & NS \\
\hline 30 & 0.14 & NS \\
\hline 27 & 0.12 & NS \\
\hline 36 & 0.17 & NS \\
\hline 32 & 0.15 & NS \\
\hline 35 & 0.17 & NS \\
\hline 33 & 0.16 & NS \\
\hline 39 & 0.16 & NS \\
\hline 33 & 0.14 & NS \\
\hline 22 & 0.08 & 22 \\
\hline 24 & 0.09 & 24 \\
\hline 25 & 0.09 & 25 \\
\hline 27 & 0.10 & 27 \\
\hline 22 & 0.08 & 22 \\
\hline
\end{tabular}




\begin{tabular}{|c|c|c|c|c|c|c|c|c|c|c|c|c|c|c|c|c|c|c|c|}
\hline $\begin{array}{l}\text { Well } \\
\text { I.D. }\end{array}$ & Date & $\begin{array}{l}\text { Depth } \\
\text { (feet) }\end{array}$ & $\begin{array}{l}\text { Percent } \\
\text { Gravel }\end{array}$ & $\begin{array}{l}\text { Percent } \\
\text { Sand }\end{array}$ & $\begin{array}{c}\text { Percent } \\
\text { Clay }\end{array}$ & & $\begin{array}{c}\text { FINAL } \\
\mathbf{t} 1 \\
\mathrm{~g}\end{array}$ & $\begin{array}{l}2 \\
\mathrm{~g}\end{array}$ & $\begin{array}{c}\text { dry } \\
\text { avg } \\
\text { g }\end{array}$ & $\begin{array}{c}\text { Initial } \\
\text { total } \\
\mathbf{g}\end{array}$ & $\begin{array}{l}\text { vial } \\
\text { \&top } \\
\text { g }\end{array}$ & $\begin{array}{c}\text { moist } \\
\mathrm{g}\end{array}$ & $\begin{array}{c}\text { Init } \\
\% \\
\text { Sat } \\
\text { wgt }\end{array}$ & $\mathbf{n}$ & $\begin{array}{c}\text { bulk } \\
\text { vol } \\
\text { cc }\end{array}$ & $\begin{array}{c}S \\
\text { percent } \\
\text { sat. } \\
\%\end{array}$ & $\begin{array}{c}Q \\
\mathrm{Vw} / \mathrm{Vt}\end{array}$ & $\begin{array}{l}>75 \% \\
\text { sand\& } \\
\text { gravel }\end{array}$ & $\begin{array}{c}<75 \\
\text { sand\& } \\
\text { gravel }\end{array}$ \\
\hline HV 21 & $11 / 13$ & 51 & 0 & 90 & 10 & SD & 35.932 & 35.492 & 5.283 & 35.932 & 30.429 & 5.50 & 4 & 0.363 & 3.14 & 19 & 0.07 & 19 & \\
\hline HV 21 & $11 / 13$ & 53 & 0 & 90 & 10 & SD & 35.916 & 35.512 & 5.271 & 35.916 & 30.443 & 5.47 & 4 & 0.363 & 3.13 & 18 & 0.06 & 18 & \\
\hline HV 21 & $11 / 13$ & 53 & 0 & 90 & 10 & SD & 36.631 & 35.998 & 5.857 & 36.631 & 30.458 & 6.17 & 5 & 0.363 & 3.48 & 25 & 0.09 & 25 & \\
\hline HV 22 & $11 / 19$ & 25 & 5 & 75 & 20 & SD & 25.425 & 25.419 & 4.285 & 25.927 & 21.137 & 4.79 & 11 & 0.376 & 2.60 & 52 & 0.19 & 52 & \\
\hline AHV 22 & $11 / 19$ & 25 & 5 & 75 & 20 & SD & 26.034 & 26.026 & 4.979 & 26.690 & 21.051 & 5.64 & 12 & 0.376 & 3.02 & 58 & 0.22 & 58 & \\
\hline MHV 22 & $11 / 19$ & 27 & 5 & 75 & 20 & SD & 26.511 & 26.502 & 5.358 & 27.247 & 21.149 & 6.10 & 12 & 0.376 & 3.25 & 61 & 0.23 & 61 & \\
\hline MHV 22 & $11 / 19$ & 27 & 5 & 75 & 20 & SD & 26.525 & 26.516 & 5.361 & 27.242 & 21.160 & 6.08 & 12 & 0.376 & 3.25 & 59 & 0.22 & 59 & \\
\hline MHV 22 & $11 / 19$ & 29 & 5 & 75 & 0 & SD & 26.493 & 26.481 & 5.361 & 28.226 & 21.126 & 7.10 & 24 & 0.376 & 3.25 & 142 & 0.53 & 142 & \\
\hline MHV 22 & $11 / 19$ & 29 & 5 & 75 & 20 & SD & 25.907 & 25.897 & 4.607 & 27.146 & 21.295 & 5.85 & 21 & 0.376 & 2.80 & 118 & 0.44 & 118 & \\
\hline HV 22 & $11 / 19$ & 31 & 3 & 72 & 25 & CLSD & 26.184 & 26.174 & 5.087 & 26.763 & 21.092 & 5.67 & 10 & 0.383 & 3.12 & 49 & 0.19 & 49 & \\
\hline HV 22 & $11 / 19$ & 31 & 3 & 72 & 25 & CLSD & 26.708 & 26.699 & 5.639 & 27.391 & 21.065 & 6.33 & 11 & 0.383 & 3.46 & 52 & 0.20 & 52 & \\
\hline HV 22 & $11 / 19$ & 33 & 5 & 75 & 20 & SD & 26.597 & 26.589 & 5.463 & 27.366 & 21.130 & 6.24 & 12 & 0.376 & 3.32 & 62 & 0.23 & 62 & \\
\hline IHV 22 & $11 / 19$ & 33 & 5 & 75 & 20 & SD & 26.501 & 26.496 & 5.371 & 27.240 & 21.128 & 6.11 & 12 & 0.376 & 3.26 & 60 & 0.23 & 60 & \\
\hline AHV 22 & $11 / 19$ & 35 & 5 & 75 & 20 & SD & 27.453 & 27.442 & 6.329 & 28.591 & 21.119 & 7.47 & 15 & 0.376 & 3.84 & 79 & 0.30 & 79 & \\
\hline IHV 22 & $11 / 19$ & 35 & 5 & 75 & 20 & SD & 26.616 & 26.608 & 5.511 & 27.951 & 21.101 & 6.85 & 20 & 0.376 & 3.35 & 106 & 0.40 & 106 & \\
\hline HV 22 & $11 / 19$ & 36 & C & 25 & 75 & SDCL & 26.637 & 26.628 & 5.507 & 27.832 & 21.126 & 6.71 & 18 & 0.448 & 3.81 & 70 & 0.31 & NS & \\
\hline HV 22 & $11 / 19$ & 36 & c & 25 & 75 & SDCL & 27.799 & 27.790 & 6.795 & 29.354 & 21.000 & 8.35 & 19 & 0.448 & 4.71 & 74 & 0.33 & vS & \\
\hline HV 22 & $11 / 19$ & 37 & 0 & 25 & 75 & SDCL & 27.437 & 27.428 & 6.429 & 28.752 & 21.004 & 7.75 & 17 & 0.448 & 4.45 & 66 & 0.30 & vS & \\
\hline HV 22 & $11 / 19$ & 37 & c & 25 & 75 & SDCL & 27.685 & 27.678 & 6.724 & 29.101 & 20.958 & 8.14 & 17 & 0.448 & 4.66 & 68 & 0.30 & vS & \\
\hline HV 22 & $11 / 19$ & 38 & ( & 40 & 60 & SDCL & 25.832 & 25.824 & 4.748 & 26.876 & 21.080 & 5.80 & 18 & 0.428 & 3.17 & 77 & 0.33 & NS & \\
\hline HV 22 & $11 / 19$ & 38 & ( & 40 & 60 & SDCL & 26.122 & 26.110 & 5.098 & 27.396 & 21.018 & 6.38 & 20 & 0.428 & 3.40 & 88 & 0.38 & NS & \\
\hline HV 22 & $11 / 19$ & 39 & t & 60 & 40 & CLSD & 27.679 & 27.669 & 6.489 & 28.780 & 21.185 & 7.60 & 15 & 0.402 & 4.13 & 67 & 0.27 & NS & \\
\hline HV 22 & $11 / 19$ & 39 & C & 60 & 40 & CLSD & 25.584 & 25.575 & 4.427 & 26.378 & 21.153 & 5.23 & 15 & 0.402 & 2.81 & 71 & 0.28 & NS & \\
\hline HV 22 & $11 / 19$ & 40 & c & 60 & 40 & CLSD & 27.054 & 27.043 & 5.909 & 28.165 & 21.140 & 7.03 & 16 & 0.402 & 3.76 & 74 & 0.30 & NS & \\
\hline IHV 22 & $11 / 19$ & 40 & 0 & 60 & 40 & CLSD & 27.771 & 27.763 & 6.603 & 28.992 & 21.164 & 7.83 & 16 & 0.402 & 4.20 & 73 & 0.29 & NS & \\
\hline IHV 22 & $11 / 19$ & 41 & 0 & 70 & 30 & CLSD & 26.970 & 26.962 & 5.854 & 27.948 & 21.112 & 6.84 & 14 & 0.389 & 3.64 & 69 & 0.27 & NS & \\
\hline AHV 22 & $11 / 19$ & 41 & 0 & 70 & 30 & CLSD & 27.251 & 27.239 & 6.052 & 28.268 & 21.193 & 7.08 & 14 & 0.389 & 3.76 & 70 & 0.27 & NS & \\
\hline AHV 22 & $11 / 19$ & 42 & 0 & 75 & 25 & CLSD & 26.983 & 26.974 & 5.865 & 27.743 & 21.114 & 6.63 & 12 & 0.383 & 3.60 & 56 & 0.21 & 56 & \\
\hline HV 22 & $11 / 19$ & 42 & 0 & 75 & 25 & CLSD & 25.652 & 25.643 & 4.597 & 26.242 & 21.0 & 5.19 & 11 & 0.383 & 2.82 & 55 & 0.21 & 55 & \\
\hline HV 22 & $/ 19$ & 43 & 2 & 78 & 20 & SD & 26.889 & 26.880 & 5.656 & 27.430 & 21.229 & 6.20 & 9 & 0.376 & 3.43 & 42 & 0.16 & 42 & \\
\hline HV 22 & $11 / 19$ & 43 & 2 & 78 & 21 & SD & 27.062 & 27.055 & 5.894 & 27.793 & 21.165 & 6.63 & 11 & 0.376 & 3.58 & 55 & 0.21 & 55 & \\
\hline HV 22 & $11 / 19$ & 44 & 2 & 88 & 1 & SD & 26.962 & 26.954 & 5.725 & 27.878 & 21.233 & 6.65 & 14 & 0.363 & 3.40 & 75 & 0.27 & 75 & \\
\hline HV 22 & $11 / 19$ & 44 & & 88 & 10 & SD & 27.076 & 27.068 & 5.907 & 28.032 & 21.165 & 6.87 & 14 & 0.363 & 3.51 & 75 & 0.27 & 75 & \\
\hline IHV 22 & $11 / 19$ & 45 & 2 & 73 & 25 & CLSD & 26.990 & 26.980 & 5.925 & 28.205 & 21.060 & 7.15 & 17 & 0.383 & 3.64 & 88 & 0.34 & 88 & \\
\hline IHV 22 & $11 / 19$ & 45 & 2 & 73 & 25 & CLSD & 26.910 & 26.901 & 5.705 & 28.235 & 21.201 & 7.03 & 19 & 0.383 & 3.50 & 99 & 0.38 & 99 & \\
\hline IHV 22 & $11 / 19$ & 47 & 0 & 70 & 30 & CLSD & 26.920 & 26.912 & 5.993 & 27.713 & 20.923 & 6.79 & 12 & 0.389 & 3.72 & 55 & 0.21 & NS & \\
\hline
\end{tabular}


Well

I.D.

$\begin{array}{llrrr}\text { MHV 22 } & 11 / 19 & 47 & 0 & 70 \\ \text { MHV 22 } & 11 / 19 & 49 & 0 & 60 \\ \text { MHV 22 } & 11 / 19 & 49 & 0 & 60 \\ \text { MHV 22 } & 11 / 19 & 51 & 0 & 92 \\ \text { MHV 22 } & 11 / 19 & 51 & 0 & 92 \\ \text { MHV 22 } & 11 / 19 & 53 & 0 & 92 \\ \text { MHV 22 } & 11 / 19 & 53 & 0 & 92 \\ & & & & \\ \text { MHV 24 } & 11 / 17 & 25 & 10 & 70 \\ \text { MHV 24 } & 11 / 17 & 25 & 10 & 70 \\ \text { MHV 24 } & 11 / 17 & 27 & 5 & 75 \\ \text { MHV 24 } & 11 / 17 & 27 & 5 & 75 \\ \text { MHV 24 } & 11 / 17 & 29 & 10 & 70 \\ \text { MHV 24 } & 11 / 17 & 29 & 10 & 70 \\ \text { MHV 24 } & 11117 & 31 & 10 & 75 \\ \text { MHV 24 } & 11 / 17 & 31 & 10 & 75 \\ \text { MHV 24 } & 11 / 17 & 33 & 0 & 5 \\ \text { MHV 24 } & 11 / 17 & 33 & 0 & 5 \\ \text { MHV 24 } & 11 / 17 & 35 & 0 & 5 \\ \text { MHV 24 } & 11 / 17 & 35 & 0 & 5 \\ \text { MHV 24 } & 11 / 17 & 36 & 0 & 20 \\ \text { MHV 24 } & 11 / 17 & 36 & 0 & 20 \\ \text { MHV 24 } & 11 / 17 & 37 & 0 & 10 \\ \text { MHV 24 } & 11 / 17 & 37 & 0 & 10 \\ \text { MHV 24 } & 11 / 17 & 38 & 0 & 20 \\ \text { MHV 24 } & 11 / 17 & 38 & 0 & 20 \\ \text { MHV 24 } & 11 / 17 & 39 & 0 & 10 \\ \text { MHV 24 } & 11 / 17 & 39 & 0 & 10 \\ \text { MHV 24 } & 11 / 17 & 40 & 0 & 5 \\ \text { MHV 24 } & 11 / 17 & 40 & 0 & 5 \\ \text { MHV 24 } & 11 / 17 & 41 & 0 & 5 \\ \text { MHV 24 } & 11 / 17 & 41 & 0 & 5 \\ \text { MHV 24 } & 11 / 17 & 42 & 0 & 10 \\ \text { MHV 24 } & 11 / 17 & 42 & 0 & 10 \\ \text { MHV 24 } & 11 / 17 & 43 & 0.1 & 15 \\ \text { MHV 24 } & 11 / 17 & 43 & 0.1 & 15 \\ \text { MHV 24 } & 11 / 17 & 44 & 2 & 88 \\ & & & & \end{array}$

FINAL t1 g $27.398 \quad 27.389$

30 CLSD 40 CLSD 40 CLSD 8 SD 8 SD 8 SD

8 SD

20 SD

20 SD

20 SD

20 SD

20 SD

20 SD

15 SD

15 SD

$95 \mathrm{CL}$

$95 \mathrm{CL}$

$95 \mathrm{CL}$

$95 \mathrm{CL}$

$80 \mathrm{CL}$

$80 \mathrm{CL}$

$90 \mathrm{CL}$

$90 \mathrm{CL}$

$80 \mathrm{CL}$

$80 \mathrm{CL}$

$90 \mathrm{CL}$

$90 \mathrm{CL}$

$95 \mathrm{CL}$

$95 \mathrm{CL}$

$95 \mathrm{CL}$

$95 \mathrm{CL}$

$90 \mathrm{CL}$

90 CL

$85 \mathrm{CL}$

$85 \mathrm{CL}$

10 SD
Init $\mathbf{n}$

$\begin{array}{llllll} & 8.035 & 21.140\end{array}$ $\begin{array}{llllll}26.330 & 26.326 & 5.189 & 26.913 & 21.139\end{array}$ $\begin{array}{llllll}27.311 & 27.305 & 6.080 & 27.950 & 21.228\end{array}$ $\begin{array}{llllll}26.949 & 26.942 & 5.908 & 27.575 & 21.038\end{array}$ $\begin{array}{llllll}26.641 & 26.635 & 5.594 & 27.271 & 21.044\end{array}$ $\begin{array}{lllll}26.323 & 26.317 & 5.149 & 26.865 & 21.171\end{array}$

$\begin{array}{lllll}26.642 & 26.641 & 5.550 & 27.502 & 21.092\end{array}$ $\begin{array}{llllll}26.661 & 26.657 & 5.556 & 27.587 & 21.103\end{array}$ $\begin{array}{llllll}26.833 & 26.830 & 5.783 & 27.924 & 21.049\end{array}$ $\begin{array}{llllll}26.365 & 26.360 & 5.273 & 27.380 & 21.090\end{array}$ $\begin{array}{llllll}26.385 & 26.380 & 5.287 & 27.073 & 21.096\end{array}$ $\begin{array}{llllll}26.178 & 26.172 & 4.966 & 26.811 & 21.209\end{array}$ $\begin{array}{llllll}26.665 & 26.661 & 5.389 & 27.353 & 21.274\end{array}$ $\begin{array}{lllll}26.090 & 26.083 & 5.170 & 26.786 & 20.917\end{array}$ $\begin{array}{llllll}27.731 & 27.730 & 6.515 & 29.396 & 21.216\end{array}$ $\begin{array}{llllll}26.115 & 26.113 & 5.065 & 27.444 & 21.049\end{array}$ $\begin{array}{lllll}26.150 & 26.146 & 4.998 & 27.423 & 21.150\end{array}$ $\begin{array}{llllll}26.622 & 26.617 & 5.538 & 27.988 & 21.082\end{array}$ $\begin{array}{llllll}25.630 & 25.626 & 4.541 & 26.883 & 21.087\end{array}$ $\begin{array}{lllll}24.941 & 24.937 & 3.749 & 26.020 & 21.190\end{array}$ $\begin{array}{lllll}26.605 & 26.602 & 5.411 & 28.085 & 21.193\end{array}$ $\begin{array}{llllll}27.315 & 27.311 & 6.080 & 28.813 & 21.233\end{array}$ $\begin{array}{llllll}27.138 & 27.134 & 6.052 & 28.923 & 21.084\end{array}$ $\begin{array}{llllll}26.492 & 26.486 & 5.423 & 27.967 & 21.066\end{array}$ $\begin{array}{lllll}26.196 & 26.193 & 5.081 & 27.807 & 21.114\end{array}$ $\begin{array}{llllll}25.191 & 25.187 & 4.017 & 26.437 & 21.172\end{array}$ $\begin{array}{llllll}25.435 & 25.432 & 4.311 & 26.794 & 21.123\end{array}$ $\begin{array}{lllll}25.911 & 25.909 & 4.787 & 27.338 & 21.123\end{array}$ $\begin{array}{lllllll}27.529 & 27.525 & 6.372 & 29.121 & 21.155\end{array}$ $\begin{array}{lllll}27.281 & 27.280 & 6.160 & 28.826 & 21.121\end{array}$ $\begin{array}{llllll}26.908 & 26.906 & 5.813 & 28.684 & 21.094\end{array}$ $\begin{array}{llllll}25.235 & 25.229 & 4.164 & 26.453 & 21.068\end{array}$ $\begin{array}{llllll}26.941 & 26.937 & 5.836 & 28.308 & 21.103\end{array}$ $\begin{array}{llllll}26.595 & 26.590 & 5.605 & 27.859 & 20.988\end{array}$ $\begin{array}{lllll}26.354 & 26.348 & 5.286 & 26.983 & 21.065\end{array}$
$\%$

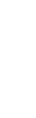

g Sat

g wgt

$\begin{array}{llll}6.90 & 9 & 0.389 & 3.88\end{array}$

$\begin{array}{llll}5.56 & 10 & 0.402 & 3.18\end{array}$

$\begin{array}{llll}5.77 & 10 & 0.402 & 3.30\end{array}$

$\begin{array}{lllll}6.72 & 10 & 0.360 & 3.59\end{array}$

$\begin{array}{lllll}6.54 & 10 & 0.360 & 3.49\end{array}$

$\begin{array}{llll}6.23 & 10 & 0.360 & 3.31\end{array}$

$\begin{array}{lllll}5.69 & 10 & 0.360 & 3.04\end{array}$

$\begin{array}{llll}6.41 & 13 & 0.376 & 3.37\end{array}$

$\begin{array}{lllll}6.48 & 14 & 0.376 & 3.37\end{array}$

$\begin{array}{lllll}6.88 & 16 & 0.376 & 3.51\end{array}$

$\begin{array}{llll}6.29 & 16 & 0.376 & 3.20\end{array}$

$\begin{array}{llll}5.98 & 12 & 0.376 & 3.21\end{array}$

$\begin{array}{llll}5.60 & 11 & 0.376 & 3.01\end{array}$

$\begin{array}{llll}6.08 & 11 & 0.370 & 3.23\end{array}$

$\begin{array}{llll}5.87 & 12 & 0.370 & 3.10\end{array}$

$\begin{array}{lllll}8.18 & 20 & 0.474 & 4.75\end{array}$

$\begin{array}{llll}6.40 & 21 & 0.474 & 3.70\end{array}$

$\begin{array}{llll}6.27 & 20 & 0.474 & 3.65\end{array}$

$\begin{array}{llll}6.91 & 20 & 0.474 & 4.04\end{array}$

$\begin{array}{llll}5.80 & 22 & 0.454 & 3.19\end{array}$

$\begin{array}{llll}4.83 & 22 & 0.454 & 2.63\end{array}$

$\begin{array}{llll}6.89 & 21 & 0.467 & 3.90\end{array}$

$\begin{array}{llll}7.58 & 20 & 0.467 & 4.38\end{array}$

$\begin{array}{llll}7.84 & 23 & 0.454 & 4.25\end{array}$

$\begin{array}{lllll}6.90 & 21 & 0.454 & 3.81\end{array}$

$\begin{array}{llll}6.69 & 24 & 0.467 & 3.66\end{array}$

$\begin{array}{lllll}5.27 & 24 & 0.467 & 2.89\end{array}$

$\begin{array}{llll}5.67 & 24 & 0.474 & 3.15\end{array}$

$\begin{array}{llll}6.22 & 23 & 0.474 & 3.49\end{array}$

$\begin{array}{lllll}7.97 & 20 & 0.474 & 4.65\end{array}$

$\begin{array}{lllll}7.71 & 20 & 0.474 & 4.50\end{array}$

$\begin{array}{lllll}7.59 & 23 & 0.467 & 4.19\end{array}$

$\begin{array}{llll}5.39 & 23 & 0.467 & 3.00\end{array}$

$\begin{array}{llll}7.21 & 19 & 0.461 & 4.15\end{array}$

$\begin{array}{lllll}6.87 & 18 & 0.461 & 3.98\end{array}$

$\begin{array}{llll}5.92 & 11 & 0.363 & 3.14\end{array}$
S

Q $>75 \%<75$

sand\& sand\&

sat. Vw/Vt gravel gravel $\%$

$\begin{array}{lll}42 & 0.17 & \text { NS }\end{array}$

$\begin{array}{lll}43 & 0.17 & \text { NS }\end{array}$

440.18 NS

$\begin{array}{lll}50 & 0.18 & 50\end{array}$

$\begin{array}{lll}50 & 0.18 & 50\end{array}$

$\begin{array}{lll}53 & 0.19 & 53\end{array}$

$\begin{array}{lll}50 & 0.18 & 50\end{array}$

$\begin{array}{llll}68 & 0.26 & 68 & S\end{array}$

$\begin{array}{llll}73 & 0.28 & 73 & S\end{array}$

$\begin{array}{llll}83 & 0.31 & 83 & S\end{array}$

$\begin{array}{llll}85 & 0.32 & 85 & S\end{array}$

$\begin{array}{llll}57 & 0.22 & 57 & \text { S }\end{array}$

$\begin{array}{llll}56 & 0.21 & 56 & S\end{array}$

$\begin{array}{llll}58 & 0.21 & 58 & S\end{array}$

$\begin{array}{llll}61 & 0.23 & 61 & S\end{array}$

$\begin{array}{llll}74 & 0.35 & \text { NS } & 74\end{array}$

$\begin{array}{llll}76 & 0.36 & \text { NS } & 76\end{array}$

$\begin{array}{llll}74 & 0.35 & \text { NS } & 74\end{array}$

$\begin{array}{llll}72 & 0.34 & \text { NS } & 72\end{array}$

$\begin{array}{llll}87 & 0.39 & \text { NS } & 87\end{array}$

$\begin{array}{llll}91 & 0.41 & \text { NS } & 91\end{array}$

$\begin{array}{llll}81 & 0.38 & \text { NS } & 81\end{array}$

$\begin{array}{llll}73 & 0.34 & \text { NS } & 73\end{array}$

$\begin{array}{llll}93 & 0.42 & \text { NS } & 93\end{array}$

$\begin{array}{llll}86 & 0.39 & \text { NS } & 86\end{array}$

$\begin{array}{llll}94 & 0.44 & \text { NS } & 94\end{array}$

$\begin{array}{llll}92 & 0.43 & \text { NS } & 92\end{array}$

$\begin{array}{llll}91 & 0.43 & \text { NS } & 91\end{array}$

$\begin{array}{llll}86 & 0.41 & \text { NS } & 86\end{array}$

$\begin{array}{llll}72 & 0.34 & \text { NS } & 72\end{array}$

$\begin{array}{llll}73 & 0.34 & \text { NS } & 73\end{array}$

$\begin{array}{llll}91 & 0.42 & \text { NS } & 91\end{array}$

$\begin{array}{llll}87 & 0.41 & \text { NS } & 87\end{array}$

$\begin{array}{llll}72 & 0.33 & \text { NS } & 72\end{array}$

$\begin{array}{llll}69 & 0.32 & \text { NS } & 69\end{array}$

$\begin{array}{rrrr}55 & 0.20 & 55 & \text { S }\end{array}$ 
Well

I.D.

Depth Percent Percent Percent

(feet) Gravel Sand Clay

$\begin{array}{lllllrl}\text { MHV 24 } & 11 / 17 & 44 & 2 & 88 & 10 & \text { SD } \\ \text { MHV 24 } & 11 / 17 & 45 & 1 & 89 & 10 & \text { SD } \\ \text { MHV 24 } & 11 / 17 & 45 & 1 & 89 & 10 & \text { SD } \\ \text { MHV 24 } & 11 / 17 & 47 & 0 & 95 & 5 & \text { SD } \\ \text { MHV 24 } & 11 / 17 & 47 & 0 & 95 & 5 & \text { SD } \\ \text { MHV 24 } & 11 / 17 & 49 & 0 & 95 & 5 & \text { SD } \\ \text { MHV 24 } & 11 / 17 & 49 & 0 & 95 & 5 & \text { SD } \\ \text { MHV 24 } & 11 / 17 & 51 & 0 & 85 & 15 & \text { SD } \\ \text { MHV 24 } & 11 / 17 & 51 & 0 & 85 & 15 & \text { SD } \\ \text { MHV 24 } & 11 / 17 & 53 & 0 & 92 & 8 & \text { SD } \\ \text { MHV 24 } & 11 / 17 & 53 & 0 & 92 & 8 & \text { SD }\end{array}$

MHB-21 4/29 MHB-21 4/29 MHB-21 4/29 MHB-21 4/29 MHB-21 4/29 MHB-21 $4 / 29$ MHB-21 4/29 MHB-21 4/29 MHB-21 4/29 MHB-21 4/29 MHB-21 4/29 MHB-21 4/29 MHB-21 4/29 MHB-21 4/29 MHB-21 4/29 MHB-21 4/29 MHB-21 4/29 MHB-21 4/29 MHB-21 4/29 MHB-21 4/29

MHB-24 4/27 MHB-24 4/27 MHB-24 4/28

$\begin{array}{rrr}25^{\prime} & 5 & 80 \\ 25^{\prime} & 5 & 80 \\ 30^{\prime} & 5 & 80 \\ 30^{\prime} & 5 & 80 \\ 34^{\prime} & 8 & 67 \\ 34^{\prime} & 8 & 67 \\ 36^{\prime} & 0 & 15 \\ 36^{\prime} & 0 & 15 \\ 38^{\prime} & 0 & 2 \\ 38^{\prime} & 0 & 2 \\ 40^{\prime} & 0 & 20 \\ 4^{\prime} & 0 & 20 \\ 42^{\prime} & 0 & 10 \\ 42^{\prime} & 0 & 10 \\ 46^{\prime} & 1 & 69 \\ 46^{\prime} & 1 & 69 \\ 50^{\prime} & 0 & 85 \\ 50^{\prime} & 0 & 85 \\ 55^{\prime} & 0 & 85 \\ 55^{\prime} & 0 & 85 \\ & & \\ 25^{\prime} & 5 & 70 \\ 25^{\prime} & 5 & 70 \\ 30^{\prime} & 5 & 70\end{array}$

15 SD

15 SD

15 SD

15 SD

25 CLSD

25 CLSD

$85 \mathrm{CL}$

$85 \mathrm{CL}$

$98 \mathrm{CL}$

$98 \mathrm{CL}$

$80 \mathrm{CL}$

$80 \mathrm{CL}$

$90 \mathrm{CL}$

$90 \mathrm{CL}$

30 CLSD

30 CLSD

15 SD

15 SD

$15 \mathrm{SD}$

15 SD

25 CLSD

25 CLSD

25 CLSD
FINAL

dry Initial vial

Init

bulk percent

$>75 \%<75$ t1 2 avg total \&top moist Sat vol sat. Vw/Nt gravel gravel $\begin{array}{lllllllll}\text { g } & \text { g } & \text { g } & \text { g } & \text { g } & \text { g } & \text { wgt } & \text { cc } & \%\end{array}$ $\begin{array}{lllllllll}26.491 & 26.483 & 5.337 & 27.310 & 21.150 & 6.16 & 13 & 0.363 & 3.17\end{array}$ $\begin{array}{llllllllll}27.371 & 27.363 & 6.237 & 28.060 & 21.130 & 6.93 & 10 & 0.363 & 3.70\end{array}$ $\begin{array}{lllllllll}26.332 & 26.327 & 5.296 & 26.832 & 21.034 & 5.80 & 9 & 0.363 & 3.14\end{array}$ $\begin{array}{llllllllll}27.644 & 27.638 & 6.523 & 28.378 & 21.118 & 7.26 & 10 & 0.357 & 3.83\end{array}$ $\begin{array}{lllllllll}25.365 & 25.357 & 4.395 & 25.833 & 20.966 & 4.87 & 10 & 0.357 & 2.58\end{array}$ $\begin{array}{lllll}26.885 & 26.879 & 5.752 & 27.443 & 21.130\end{array}$ $\begin{array}{lllll}26.722 & 26.715 & 5.664 & 27.235 & 21.055\end{array}$ $\begin{array}{lllll}26.607 & 26.602 & 5.343 & 27.108 & 21.262\end{array}$ $\begin{array}{lllll}27.109 & 27.103 & 6.068 & 27.712 & 21.038\end{array}$ $\begin{array}{lllll}26.993 & 26.986 & 5.742 & 27.434 & 21.248\end{array}$ $\begin{array}{lllll}26.872 & 26.865 & 5.671 & 27.353 & 21.198\end{array}$

$\begin{array}{llll}6.31 & 9 & 0.357 & 3.38\end{array}$

$\begin{array}{llll}6.18 & 8 & 0.357 & 3.32\end{array}$

$\begin{array}{llll}5.85 & 9 & 0.370 & 3.21\end{array}$

$\begin{array}{llll}6.67 & 9 & 0.370 & 3.64\end{array}$

$\begin{array}{llll}6.19 & 7 & 0.360 & 3.39\end{array}$

$\begin{array}{lllll}6.16 & 8 & 0.360 & 3.35\end{array}$

$\begin{array}{llll}72 & 0.26 & 72 & \text { S } \\ 52 & 0.19 & 52 & \text { S } \\ 44 & 0.16 & 44 & \text { S } \\ 54 & 0.19 & 54 & \text { S } \\ 51 & 0.18 & 51 & \text { S } \\ 47 & 0.17 & 47 & \text { S } \\ 44 & \mathbf{0 . 1 6} & \mathbf{4 4} & \text { S } \\ \mathbf{4 2} & \mathbf{0 . 1 6} & \mathbf{4 2} & \mathbf{S} \\ \mathbf{4 5} & \mathbf{0 . 1 7} & \mathbf{4 5} & \mathbf{S} \\ 36 & \mathbf{0 . 1 3} & \mathbf{3 6} & \mathbf{S} \\ \mathbf{4 0} & \mathbf{0 . 1 4} & \mathbf{4 0} & \mathrm{S}\end{array}$

$\begin{array}{lllllllll}49.170 & 49.169 & 17.399 & 52.830 & 31.771 & 21.06 & 17 & 0.370 & 10.44\end{array}$ $\begin{array}{lllllllll}52.194 & 52.155 & 19.410 & 55.902 & 32.764 & 23.14 & 16 & 0.370 & 11.65\end{array}$ $\begin{array}{llllllllll}47.552 & 47.557 & 15.358 & 49.606 & 32.196 & 17.41 & 12 & 0.370 & 9.22\end{array}$ $\begin{array}{llllllllll}48.503 & 48.509 & 15.750 & 50.642 & 32.756 & 17.89 & 12 & 0.370 & 9.45\end{array}$ $\begin{array}{lllllllll}53.640 & 53.596 & 21.704 & 57.653 & 31.914 & 25.74 & 16 & 0.383 & 13.33\end{array}$ $\begin{array}{lllllllll}50.265 & 50.258 & 18.108 & 53.873 & 32.153 & 21.72 & 17 & 0.383 & 11.12\end{array}$ $\begin{array}{lllllllll}51.790 & 51.568 & 19.264 & 55.681 & 32.414 & 23.27 & 17 & 0.461 & 13.69\end{array}$ $\begin{array}{lllllllll}54.982 & 54.667 & 21.603 & 59.375 & 33.221 & 26.15 & 17 & 0.461 & 15.36\end{array}$ $\begin{array}{lllllllll}55.997 & 55.970 & 23.040 & 60.891 & 32.943 & 27.95 & 18 & 0.477 & 16.95\end{array}$ $\begin{array}{llllllllll}51.273 & 51.104 & 18.660 & 55.369 & 32.528 & 22.84 & 18 & 0.477 & 13.73\end{array}$ $\begin{array}{lllllllll}52.237 & 52.094 & 19.559 & 56.686 & 32.607 & 24.08 & 19 & 0.454 & 13.72\end{array}$ $\begin{array}{llllllllll}55.010 & 54.314 & 22.617 & 59.778 & 32.045 & 27.73 & 18 & 0.454 & 15.87\end{array}$ $\begin{array}{lllllllll}56.495 & 55.638 & 23.697 & 60.990 & 32.370 & 28.62 & 17 & 0.467 & 17.07\end{array}$ $\begin{array}{lllllllll}51.810 & 51.713 & 19.746 & 56.305 & 32.015 & 24.29 & 19 & 0.467 & 14.22\end{array}$ $\begin{array}{llllllllll}56.281 & 56.281 & 23.130 & 59.020 & 33.151 & 25.87 & 11 & 0.389 & 14.37\end{array}$ $\begin{array}{lllllllll}54.546 & 54.511 & 22.887 & 57.895 & 31.641 & 26.25 & 13 & 0.389 & 14.22\end{array}$ $\begin{array}{lllllllll}56.746 & 56.750 & 24.090 & 59.436 & 32.658 & 26.78 & 10 & 0.370 & 14.46\end{array}$ $\begin{array}{lllllllll}60.137 & 60.142 & 27.277 & 63.057 & 32.862 & 30.19 & 10 & 0.370 & 16.37\end{array}$ $\begin{array}{lllllllll}48.600 & 48.604 & 17.104 & 50.991 & 31.498 & 19.49 & 12 & 0.370 & 10.27\end{array}$ $\begin{array}{lllllllll}52.679 & 52.682 & 20.341 & 55.298 & 32.340 & 22.96 & 11 & 0.370 & 12.21\end{array}$

$\begin{array}{lllllllll}50.487 & 50.489 & 17.876 & 53.436 & 32.612 & 20.82 & 14 & 0.383 & 10.98\end{array}$ $\begin{array}{lllllllll}52.213 & 52.215 & 19.498 & 55.532 & 32.716 & 22.82 & 15 & 0.383 & 11.97\end{array}$ $\begin{array}{lllllllll}49.025 & 49.029 & 16.829 & 51.573 & 32.198 & 19.37 & 13 & 0.383 & 10.33\end{array}$

\begin{tabular}{|c|c|c|}
\hline 95 & 0.35 & 95 \\
\hline 87 & 0.32 & 87 \\
\hline 60 & 0.22 & 60 \\
\hline 61 & 0.23 & 61 \\
\hline 79 & 0.30 & 79 \\
\hline 85 & 0.32 & 85 \\
\hline 63 & 0.29 & NS \\
\hline 64 & 0.30 & NS \\
\hline 61 & 0.29 & NS \\
\hline 64 & 0.30 & NS \\
\hline 73 & 0.33 & NS \\
\hline 71 & 0.32 & NS \\
\hline 62 & 0.29 & NS \\
\hline 68 & 0.32 & NS \\
\hline 49 & 0.19 & NS \\
\hline 61 & 0.24 & NS \\
\hline 50 & 0.19 & 50 \\
\hline 48 & 0.18 & 48 \\
\hline 63 & 0.23 & 63 \\
\hline 58 & 0.21 & 58 \\
\hline 70 & 0.27 & 70 \\
\hline 72 & 0.28 & 72 \\
\hline 64 & 0.25 & 64 \\
\hline
\end{tabular}




\begin{tabular}{|c|c|c|c|c|c|c|c|c|c|c|c|c|c|c|c|c|c|c|c|}
\hline $\begin{array}{l}\text { Well } \\
\text { I.D. }\end{array}$ & Date & $\begin{array}{l}\text { Depth } \\
\text { (feet) }\end{array}$ & $\begin{array}{l}\text { Percent } \\
\text { Gravel }\end{array}$ & $\begin{array}{l}\text { Percent } \\
\text { Sand }\end{array}$ & $\begin{array}{c}\text { Percent } \\
\text { Clay }\end{array}$ & & $\begin{array}{c}\text { FINAL } \\
\mathbf{t 1} \\
\mathbf{g}\end{array}$ & $\begin{array}{l}\mathbf{2} \\
\mathbf{g}\end{array}$ & $\begin{array}{c}\text { dry } \\
\text { avg } \\
\text { g }\end{array}$ & $\begin{array}{c}\text { Initial } \\
\text { total } \\
\mathbf{g}\end{array}$ & $\begin{array}{c}\text { vial } \\
\text { \&top } \\
\mathbf{g}\end{array}$ & $\begin{array}{c}\text { moist } \\
\text { g }\end{array}$ & $\begin{array}{c}\text { Init } \\
\% \\
\text { Sat } \\
\text { wgt }\end{array}$ & $\mathbf{n}$ & $\begin{array}{c}\text { bulk } \\
\text { vol } \\
\text { cc }\end{array}$ & $\begin{array}{c}\text { S } \\
\text { percent } \\
\text { sat. } \\
\%\end{array}$ & Vw/Vt & $\begin{array}{l}>75 \% \\
\text { sand\& } \\
\text { gravel }\end{array}$ & $\begin{array}{l}<75 \\
\text { sand\& } \\
\text { gravel }\end{array}$ \\
\hline MHB-24 & $4 / 28$ & $30^{\prime}$ & 5 & 70 & 25 & CLSD & 53.350 & 53.353 & 20.445 & & 32.906 & 23.06 & 11 & 0.383 & 12.55 & 55 & 0.21 & 55 & $\mathbf{S}$ \\
\hline MHB-24 & $4 / 28$ & 34' & $\mathbf{0}$ & 10 & 90 & $\mathrm{CL}$ & 49.820 & 49.366 & 17.489 & 53.834 & 32.104 & 21.73 & 20 & 0.467 & 12.60 & 72 & 0.34 & NS & 72 \\
\hline MHB-24 & $4 / 28$ & $34^{\prime}$ & 0 & 10 & 90 & $\mathrm{CL}$ & 50.925 & 50.822 & 18.267 & 55.592 & 32.607 & 22.99 & 21 & 0.467 & 13.16 & 77 & 0.36 & NS & $n$ \\
\hline MHB-24 & $4 / 28$ & $36^{\prime}$ & 0 & 5 & 95 & $\mathrm{CL}$ & 49.722 & 49.625 & 16.899 & 53.721 & 32.774 & 20.95 & 19 & 0.474 & 12.33 & 69 & 0.33 & NS & 69 \\
\hline MHB-24 & $4 / 28$ & $36^{\prime}$ & 0 & 5 & 95 & CL & 51.390 & 51.150 & 19.198 & 55.809 & 32.072 & 23.74 & 19 & 0.474 & 14.01 & 68 & 0.32 & NS & 68 \\
\hline MHB-24 & $4 / 28$ & 38' & $\mathbf{0}$ & 5 & 95 & CL & 54.170 & 53.874 & 21.543 & 58.440 & 32.479 & 25.96 & 17 & 0.474 & 15.72 & 59 & 0.28 & NS & 59 \\
\hline IHB-24 & $4 / 28$ & $38^{\prime}$ & 0 & 5 & 95 & CL & 52.214 & 52.124 & 19.601 & 56.369 & 32.568 & 23.80 & 18 & 0.474 & 14.31 & 62 & 0.29 & NS & 62 \\
\hline HB-24 & $4 / 28$ & $40^{\prime}$ & 0 & 20 & 80 & CL & 46.243 & 46.247 & 13.592 & 49.035 & 32.653 & 16.38 & 17 & 0.454 & 9.54 & 64 & 0.29 & NS & 64 \\
\hline HB-24 & $4 / 28$ & $40^{\prime}$ & 0 & 20 & 80 & $\mathrm{Cl}$ & 46.997 & 46.987 & 13.960 & 50.302 & 33.032 & 17.27 & 19 & 0.454 & 9.80 & 74 & 0.34 & NS & 74 \\
\hline IHB-24 & $4 / 28$ & $42^{\prime}$ & 0 & 45 & 55 & $\mathrm{CL}$ & 48.926 & 48.929 & 15.742 & 51.496 & 33.186 & 18.31 & 14 & 0.422 & 10.38 & 59 & 0.25 & NS & 59 \\
\hline MHB-24 & $4 / 28$ & 42' & 0 & 45 & 55 & $\mathrm{Cl}$ & 49.144 & 49.138 & 16.369 & 52.423 & 32.772 & 19.65 & 17 & 0.422 & 10.79 & 72 & 0.30 & NS & 72 \\
\hline MHB-24 & $4 / 28$ & $4^{\circ}$ & 0 & 45 & 55 & SDCL & 55.199 & 55.203 & 23.535 & 58.292 & 31.666 & 26.63 & 12 & 0.422 & 15.51 & 47 & 0.20 & NS & 47 \\
\hline MHB-24 & $4 / 28$ & $46^{\circ}$ & 0 & 45 & 55 & SDCL & 56.544 & 56.549 & 24.756 & 58.903 & 31.791 & 27.11 & 9 & 0.422 & 16.32 & 34 & 0.14 & NS & 34 \\
\hline MHB-24 & $4 / 28$ & $50^{\prime}$ & $\mathbf{0}$ & 75 & 25 & CLSD & 55.023 & 55.027 & 22.188 & 57.247 & 32.837 & 24.41 & 9 & 0.383 & 13.62 & 43 & 0.16 & 43 & $\mathbf{S}$ \\
\hline MHB-24 & $4 / 28$ & $50^{\circ}$ & 0 & 75 & 25 & CLSD & 51.098 & 51.101 & 18.020 & 52.694 & 33.080 & 19.61 & 8 & 0.383 & 11.06 & 38 & 0.14 & 38 & $\mathbf{S}$ \\
\hline MHB-24 & $4 / 28$ & $55^{\prime}$ & 0 & 80 & 20 & SD & 53.255 & 53.260 & 20.347 & 56.375 & 32.911 & 23.46 & 13 & 0.376 & 12.35 & 67 & 0.25 & 67 & $\mathbf{S}$ \\
\hline MHB-24 & $4 / 28$ & $55^{\circ}$ & 0 & 80 & 20 & SD & 53.049 & 53.049 & 20.495 & 56.055 & 32.554 & 23.50 & 13 & 0.376 & 12.44 & 64 & 0.24 & 64 & $\mathbf{S}$ \\
\hline HB-28 & $4 / 26$ & & 0 & 15 & 8 & CL & 2.467 & 52.289 & 19.670 & 57.541 & 32.708 & 24.83 & 21 & 0.461 & 13.98 & 80 & 0.37 & NS & \\
\hline MHB-28 & $4 / 26$ & $25^{\prime}$ & 0 & 15 & 85 & CL & 53.055 & 52.596 & 19.673 & 57.890 & 33.153 & 24.74 & 20 & 0.461 & 13.98 & 79 & 0.36 & NS & 79 \\
\hline MHB-28 & $4 / 26$ & $30^{\prime}$ & 0 & 5 & 95 & CL & 51.219 & 51.207 & 18.357 & 54.560 & 32.856 & 21.70 & 15 & 0.474 & 13.40 & 53 & 0.25 & NS & 53 \\
\hline MHB-28 & $4 / 26$ & $\sigma^{\prime}$ & 0 & 5 & 95 & $\mathrm{CL}$ & 50.981 & 50.929 & 18.642 & 54.296 & 32.313 & 21.98 & 15 & 0.474 & 13.61 & 52 & 0.25 & NS & 52 \\
\hline MHB-28 & $4 / 27$ & & 0 & 20 & 80 & CL & 52.481 & 52.009 & 19.141 & 57.907 & 33.104 & 24.80 & 23 & 0.454 & 13.43 & 93 & 0.42 & NS & 93 \\
\hline MHB-28 & $4 / 27$ & & 0 & 20 & 80 & CL & 51.754 & 51.218 & 18.610 & 56.873 & 32.876 & 24.00 & 22 & 0.454 & 13.06 & 91 & 0.41 & NS & 91 \\
\hline MHB-28 & $4 / 27$ & $r$ & $\mathbf{0}$ & 5 & 95 & $\mathrm{Cl}$ & 55.621 & 54.729 & 22.269 & 60.659 & 32.905 & 27.75 & 20 & 0.474 & 16.25 & 71 & 0.34 & NS & 71 \\
\hline MHB-28 & $4 / 27$ & & $\mathbf{0}$ & 5 & 95 & $\mathrm{Cl}$ & 53.755 & 53.328 & 20.662 & 59.015 & 32.879 & 26.14 & 21 & 0.474 & 15.08 & 77 & 0.36 & NS & 77 \\
\hline MHB-28 & $4 / 27$ & 3 & $\mathbf{0}$ & 25 & 75 & SDCL & 52.154 & 51.697 & 20.851 & 57.178 & 31.075 & 26.10 & 20 & 0.448 & 14.45 & 81 & 0.36 & NS & 81 \\
\hline MHB-28 & $4 / 27$ & 3 & $\mathbf{0}$ & 25 & 75 & SDCL & 50.778 & 50.702 & 18.181 & 55.963 & 32.559 & 23.40 & 22 & 0.448 & 12.60 & 93 & 0.41 & NS & 93 \\
\hline MHB-28 & $4 / 27$ & 43' & 0 & 10 & 90 & $\mathrm{CL}$ & 3.102 & 53.095 & 20.904 & 58.173 & 32.195 & 25.98 & 20 & 0.467 & 15.06 & 72 & 0.34 & NS & 72 \\
\hline MHB-28 & $4 / 27$ & 43 & 0 & 10 & 90 & $\mathrm{CL}$ & 4.489 & 54.453 & 21.621 & 60.322 & 32.850 & 27.47 & 21 & 0.467 & 15.57 & 80 & 0.38 & NS & 80 \\
\hline MHB-28 & $4 / 27$ & 4 & $\mathbf{0}$ & 4 & 55 & SDCL & 57.127 & 57.132 & 24.415 & 60.026 & 32.715 & 27.31 & 11 & 0.422 & 16.09 & 43 & 0.18 & NS & 43 \\
\hline MHB-28 & $4 / 27$ & & 0 & 4 & 55 & SDCL & 54.720 & 54.725 & 22.071 & 57.006 & 32.652 & 24.35 & 9 & 0.422 & 14.55 & 37 & 0.16 & NS & 37 \\
\hline HB-28 & 27 & & 0 & 6 & 35 & CLSD & 52.651 & 52.653 & 20.155 & 54.912 & 32.497 & 22.42 & 10 & 0.396 & 12.67 & 45 & 0.18 & NS & 45 \\
\hline HB-28 & $4 / 27$ & & $\mathbf{0}$ & 6 & 35 & CLSD & 52.694 & 52.696 & 20.410 & 54.727 & 32.285 & 22.44 & 9 & 0.396 & 12.83 & 40 & 0.16 & NS & 40 \\
\hline HB-28 & 27 & & 2 & 6 & 35 & CLSD & 52.814 & 52.817 & 19.886 & 54.704 & 32.930 & 21.77 & 9 & 0.396 & 12.50 & 38 & 0.15 & NS & 38 \\
\hline HB-28 & $4 /$ & & 2 & 63 & 35 & CLSD & 51.246 & 51.251 & 18.924 & 52.951 & 32.324 & 20.63 & 8 & 0.396 & 11.89 & 36 & 0.14 & NS & 36 \\
\hline MHB-28 & $4 / 27$ & $55^{\prime}$ & 0 & 92 & 8 & SD & 54.750 & 54.755 & 22.564 & 56.451 & 32.188 & 24.26 & 7 & 0.360 & 13.33 & 35 & 0.13 & 35 & $\mathbf{S}$ \\
\hline
\end{tabular}




\begin{tabular}{|c|c|c|c|c|}
\hline $\begin{array}{l}\text { Well } \\
\text { I.D. }\end{array}$ & Date & $\begin{array}{l}\text { Depth } \\
\text { (feet) }\end{array}$ & $\begin{array}{l}\text { Percent } \\
\text { Gravel }\end{array}$ & $\begin{array}{l}\text { Percent } \\
\text { Sand }\end{array}$ \\
\hline MHB-28 & $4 / 27$ & $55^{\circ}$ & 0 & 92 \\
\hline & $\begin{array}{c}\text { AVG } \\
\text { CLAY }\end{array}$ & $\begin{array}{l}\text { AVG } \\
\text { SAND }\end{array}$ & $\begin{array}{r}\text { SD } \\
\text { CLAY }\end{array}$ & $\begin{array}{r}\text { SD } \\
\text { SAND }\end{array}$ \\
\hline MHV-24 & 82 & 56 & 9 & 14 \\
\hline MHV-22 & 66 & 69 & 13 & 25 \\
\hline MHV-21 & 16 & 28 & 4 & 13 \\
\hline MHB-21 & 164 & 69 & 7 & 16 \\
\hline MHB-24 & 63 & 59 & 9 & 9 \\
\hline MHB-2 & 82 & 50 & 9 & 9 \\
\hline
\end{tabular}


Appendix III-Tabulated TCE and PCE for Pre- and Post-test Borings 


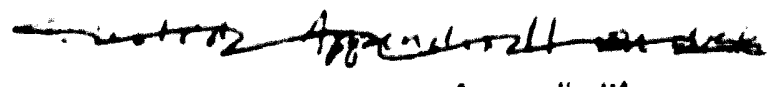

Appondix III

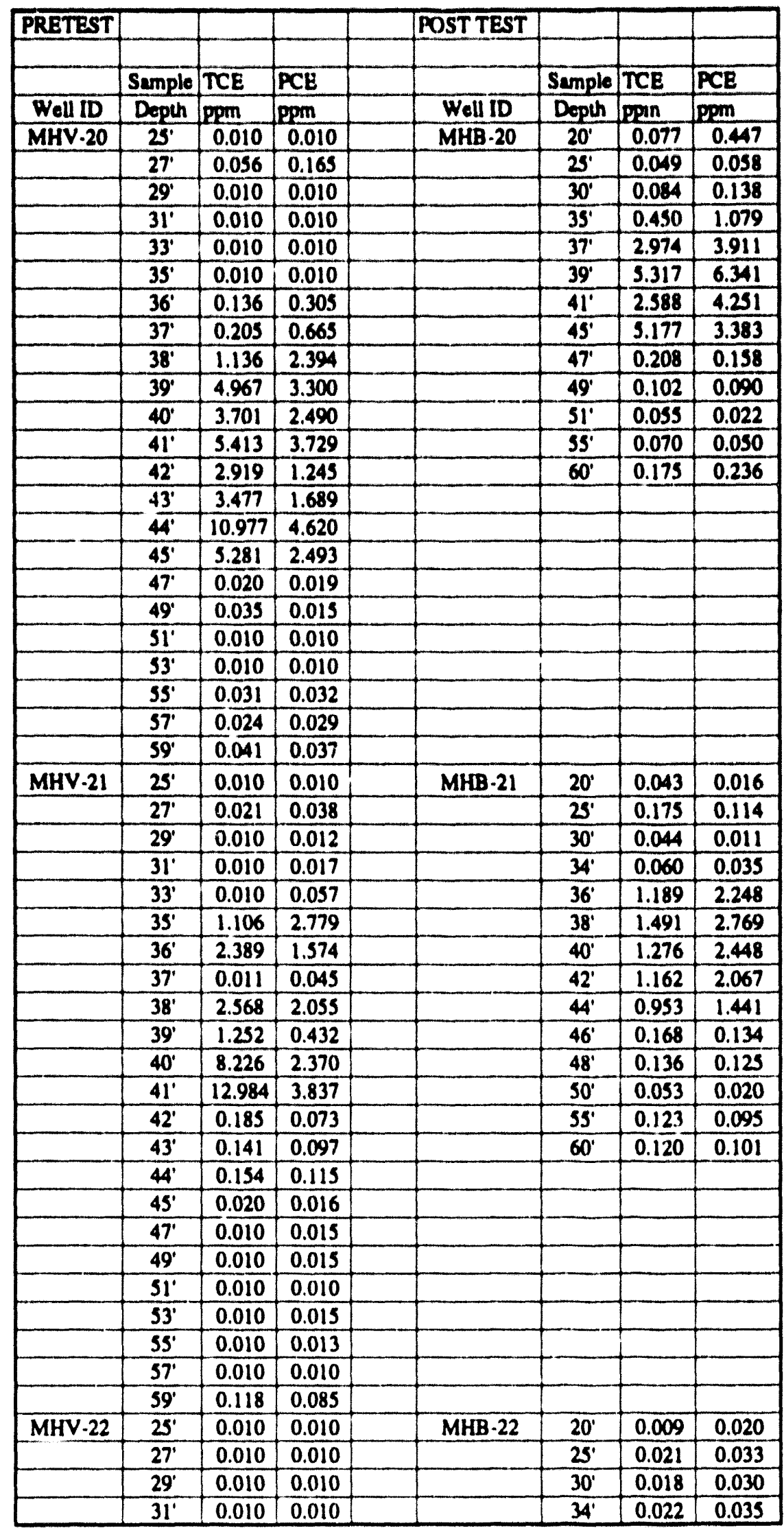




\section{Appondix II}

\begin{tabular}{|c|c|c|c|c|c|c|c|}
\hline & Semple & TCE & $\overline{P C E}$ & & Sumple & TCE & $\overline{P C E}$ \\
\hline \multirow[t]{20}{*}{ Well ID } & Depth & pem & $\mathrm{PPm}$ & Well ID & Depth & ppon & Ppm \\
\hline & $33^{\prime}$ & 0.010 & 0.010 & & $36^{\prime}$ & 0.160 & 0.543 \\
\hline & $35^{\prime}$ & 2.664 & 1.269 & & $38^{\prime}$ & 0.462 & 0.409 \\
\hline & 36 & 0.055 & 0.043 & & $40^{\prime}$ & 0.070 & 0.247 \\
\hline & 37 & 0.010 & 0.010 & & $42^{\prime}$ & 0.020 & 0.038 \\
\hline & $38^{\prime}$ & 0.010 & 0.010 & & $44^{\prime}$ & 0.147 & 0.188 \\
\hline & $39^{\prime}$ & 0.034 & 0.040 & & $46^{\circ}$ & 0.094 & 0.114 \\
\hline & $40^{\circ}$ & 0.069 & 0.117 & & $48^{\prime}$ & 0.073 & 0.097 \\
\hline & $41^{\prime}$ & 0.010 & 0.010 & & $50^{\prime}$ & 0.023 & 0.028 \\
\hline & 42 & 0.010 & 0.010 & & 55' & 0.057 & 0.051 \\
\hline & $43^{\prime}$ & 0.010 & 0.010 & & $60^{\circ}$ & 0.121 & 0.091 \\
\hline & 4 & 0.010 & 0.010 & & & & \\
\hline & $45^{\circ}$ & 0.122 & 0.096 & & & & \\
\hline & 47 & 0.010 & 0.010 & & & & \\
\hline & $49^{\circ}$ & 0.010 & 0.010 & & & & \\
\hline & $51^{\prime}$ & 0.010 & 0.010 & & & & \\
\hline & 53 & 0.010 & 0.010 & & & & \\
\hline & $55^{\prime}$ & 0.016 & 0.021 & & & & \\
\hline & $57^{\circ}$ & 0.010 & 0.013 & & & & \\
\hline & $59^{\circ}$ & 0.106 & 0.160 & & & & \\
\hline \multirow[t]{23}{*}{ MHV-23 } & 25 & 0.010 & 0.030 & MHB.23 & 20 & 0.044 & 0.109 \\
\hline & $27^{\circ}$ & 0.010 & 0.013 & & 25 & 0.060 & 0.146 \\
\hline & $29^{\circ}$ & 0.010 & 0.010 & & $30^{\prime}$ & 0.102 & 0.317 \\
\hline & 31 & 0.010 & 0.010 & & 35 & 0.040 & 0.056 \\
\hline & $33^{\circ}$ & 0.010 & 0.010 & & 37 & 0.075 & 0.309 \\
\hline & $35^{\circ}$ & 0.010 & 0.010 & & $39^{\prime}$ & 0.082 & 0.340 \\
\hline & 36 & 0.022 & 0.097 & & $41^{\prime}$ & 0.831 & 2.160 \\
\hline & 37 & 0.198 & 0.849 & & 43 & 1.935 & 4.572 \\
\hline & $38^{\prime}$ & 1.082 & 2.889 & & $45^{\circ}$ & 0.859 & 1.713 \\
\hline & $39^{\prime}$ & 0.196 & 0.330 & & 47 & 0.137 & 0.184 \\
\hline & $40^{\circ}$ & 5.229 & 5.235 & & $49^{\prime}$ & 0.055 & 0.038 \\
\hline & $41^{\prime}$ & 3.474 & 4.767 & & $51^{\circ}$ & 0.065 & 0.052 \\
\hline & $42^{\prime}$ & 3.051 & 3.611 & & $55^{\circ}$ & 0.079 & 0.058 \\
\hline & $43^{\prime}$ & 4.073 & 4.463 & & $60^{\circ}$ & 0.226 & 0.171 \\
\hline & $44^{\circ}$ & 2.528 & 0.306 & & & & \\
\hline & 45' & 0.010 & 0.010 & & & & \\
\hline & $47^{\circ}$ & 0.043 & 0.043 & & & & \\
\hline & $49^{\prime}$ & 0.010 & 0.010 & & & & \\
\hline & $51^{\prime}$ & 0.010 & 0.010 & & & & \\
\hline & 53 & 0.010 & 0.010 & & & & \\
\hline & 55 & 0.080 & 0.119 & & & & \\
\hline & 57 & 0.010 & 0.010 & & & & \\
\hline & $59^{\prime}$ & 0.111 & 0.133 & & & & \\
\hline \multirow[t]{9}{*}{ MHV.24 } & 25 & 0.010 & 0.010 & MHB. 24 & $20^{\circ}$ & 0.026 & 0.008 \\
\hline & 27 & 0.010 & 0.010 & & 25 & 0.043 & 0.014 \\
\hline & $29^{\prime}$ & 0.010 & 0.010 & & $30^{\circ}$ & 0.032 & 0.010 \\
\hline & $31^{\prime}$ & 0.010 & 0.010 & & 34' & 0.096 & 0.157 \\
\hline & $33^{\prime}$ & 0.010 & 0.011 & & $36^{\prime}$ & 0.300 & 0.600 \\
\hline & 35 & 0.230 & 0.397 & & $38^{\prime}$ & 0.184 & 0.232 \\
\hline & $36^{\circ}$ & 0.067 & 0.073 & & $40^{\circ}$ & 0.033 & 0.009 \\
\hline & 37 & 0.152 & 0.161 & & 42 & 0.204 & 0.148 \\
\hline & $38^{\circ}$ & 0.058 & 0.043 & & $44^{\prime}$ & 0.035 & 0.013 \\
\hline
\end{tabular}


Appondix II

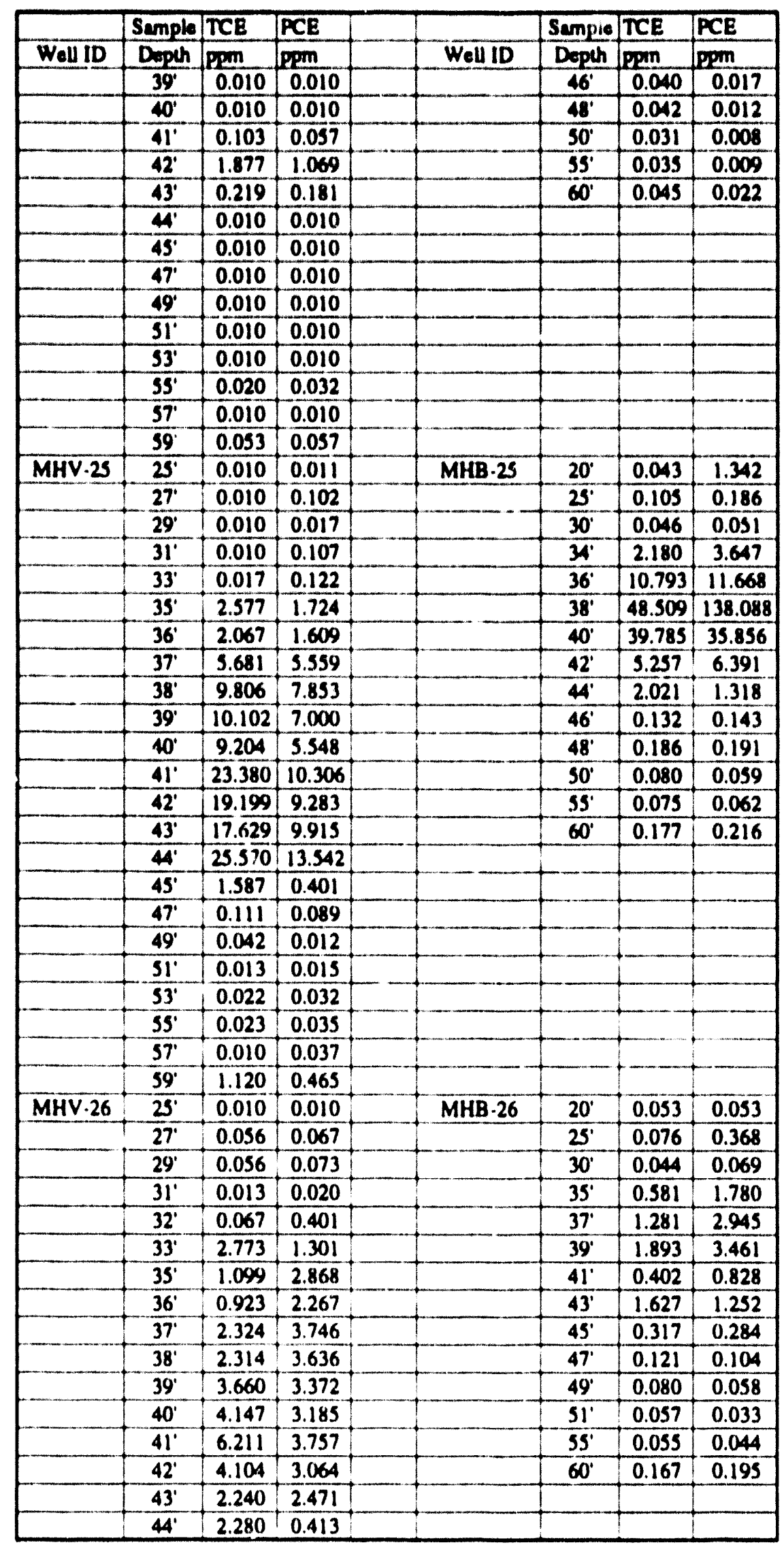


Appendix II

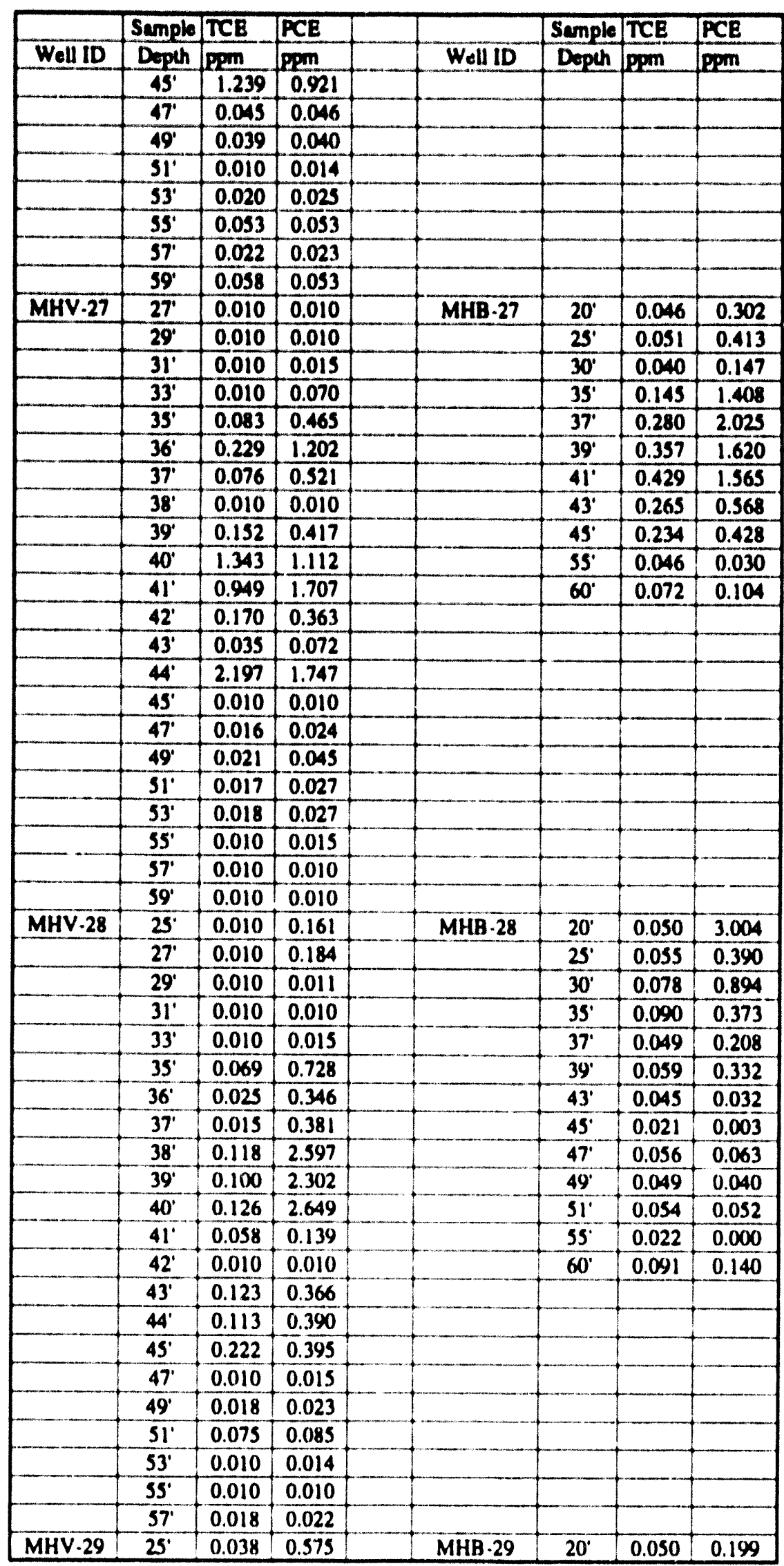


Appondix II

\begin{tabular}{|c|c|c|c|c|c|c|c|}
\hline & Semple & TCE & PCE & & Semple & TCE & PCE \\
\hline Well ID & Dopth & $\mathrm{ppm}$ & $\mathrm{ppm}$ & Well ID & Deph & $\mathrm{ppm}$ & $\mathrm{pgm}$ \\
\hline & 27 & 0.068 & 1.015 & & 25 & 0.057 & 0.721 \\
\hline & $29^{\prime}$ & 0.011 & 0.118 & & $30^{\prime}$ & 0.079 & 1.324 \\
\hline & $31^{\circ}$ & 0.024 & 0.071 & & $35^{\prime}$ & 0.212 & 1.056 \\
\hline & $33^{\prime}$ & 0.140 & 1.830 & & 37 & 0.518 & 1.821 \\
\hline & $35^{\circ}$ & 0.327 & 1.378 & & $39^{\circ}$ & 0.965 & 2.171 \\
\hline & 36 & 0.871 & 2.279 & & $41^{\prime}$ & 1.003 & 1.076 \\
\hline & 37 & 0.907 & 1.480 & & $43^{\prime}$ & 0.214 & 0.334 \\
\hline & $38^{\prime}$ & 1.076 & 1.805 & & $45^{\circ}$ & 0.149 & 0.275 \\
\hline & $39^{\circ}$ & 1.736 & 1.431 & & 47 & 0.131 & 0.236 \\
\hline & $40^{\circ}$ & 2.989 & 1.906 & & $49^{\prime}$ & 0.177 & 0.385 \\
\hline & $41^{\prime}$ & 1.466 & 1.040 & & $51^{\circ}$ & 0.053 & 0.023 \\
\hline & 42 & 2.604 & 0.869 & & 55 & 0.087 & 0.152 \\
\hline & $43^{\prime}$ & 0.204 & 0.354 & & $60^{\circ}$ & 0.372 & 1.120 \\
\hline & $44^{\prime}$ & 0.213 & 0.352 & & & & \\
\hline & 47 & 0.072 & 0.073 & & & & \\
\hline & $49^{\circ}$ & 0.010 & 0.010 & & & & \\
\hline & $51^{\circ}$ & 0.010 & 0.011 & & & & \\
\hline & $53^{\prime}$ & 0.019 & 0.020 & & & & \\
\hline & 55 & 0.018 & 0.018 & & & & \\
\hline & 57 & 0.114 & 0.101 & & & & \\
\hline & $39^{\circ}$ & 0.035 & 0.026 & & & & \\
\hline
\end{tabular}




\section{Appendix IV-Plots of TCE and PCE vs. Depth}


MHV-20 TCE Concentration

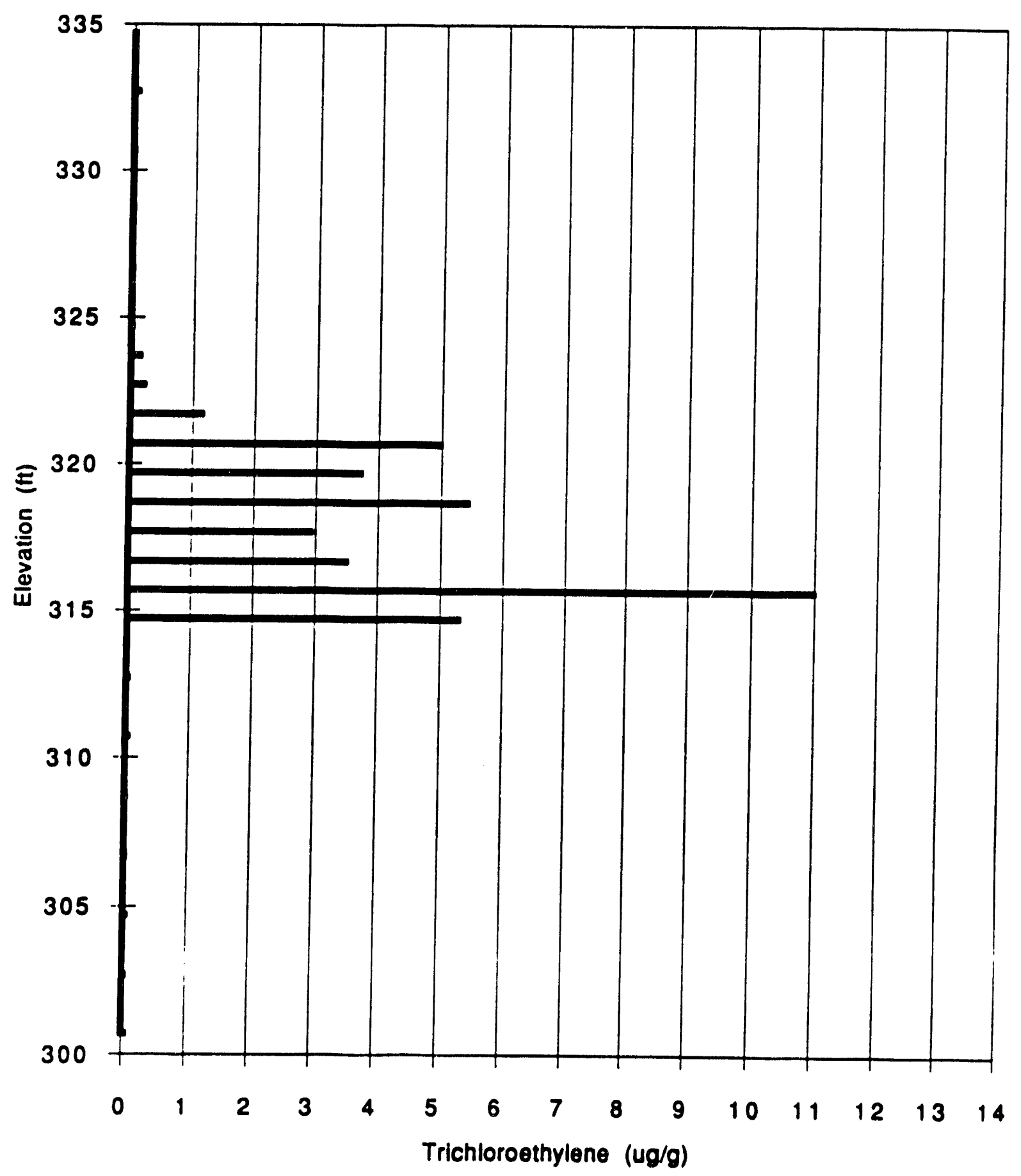


MHV-20 PCE Concentration

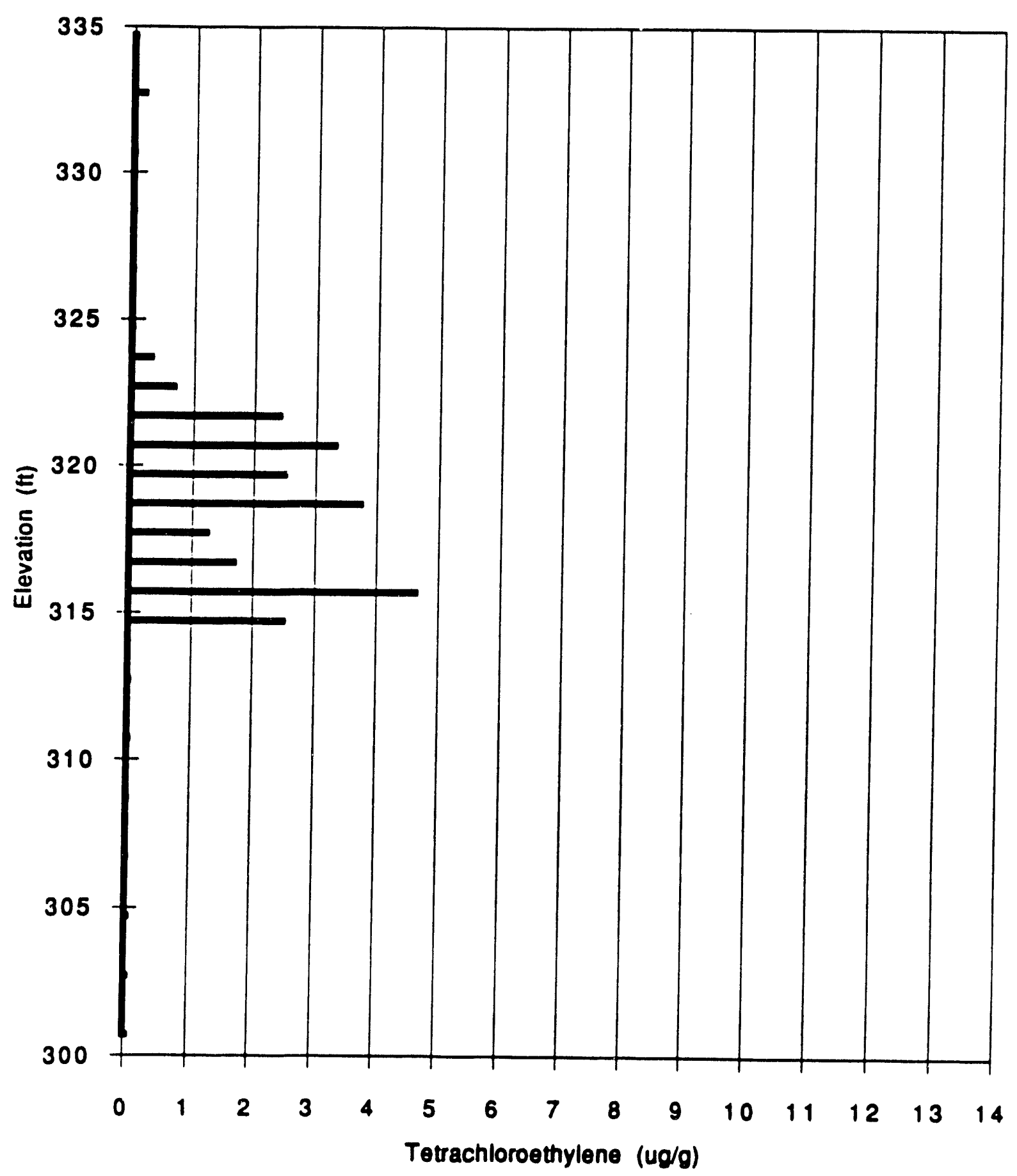


MHV-21 TCE Concentration

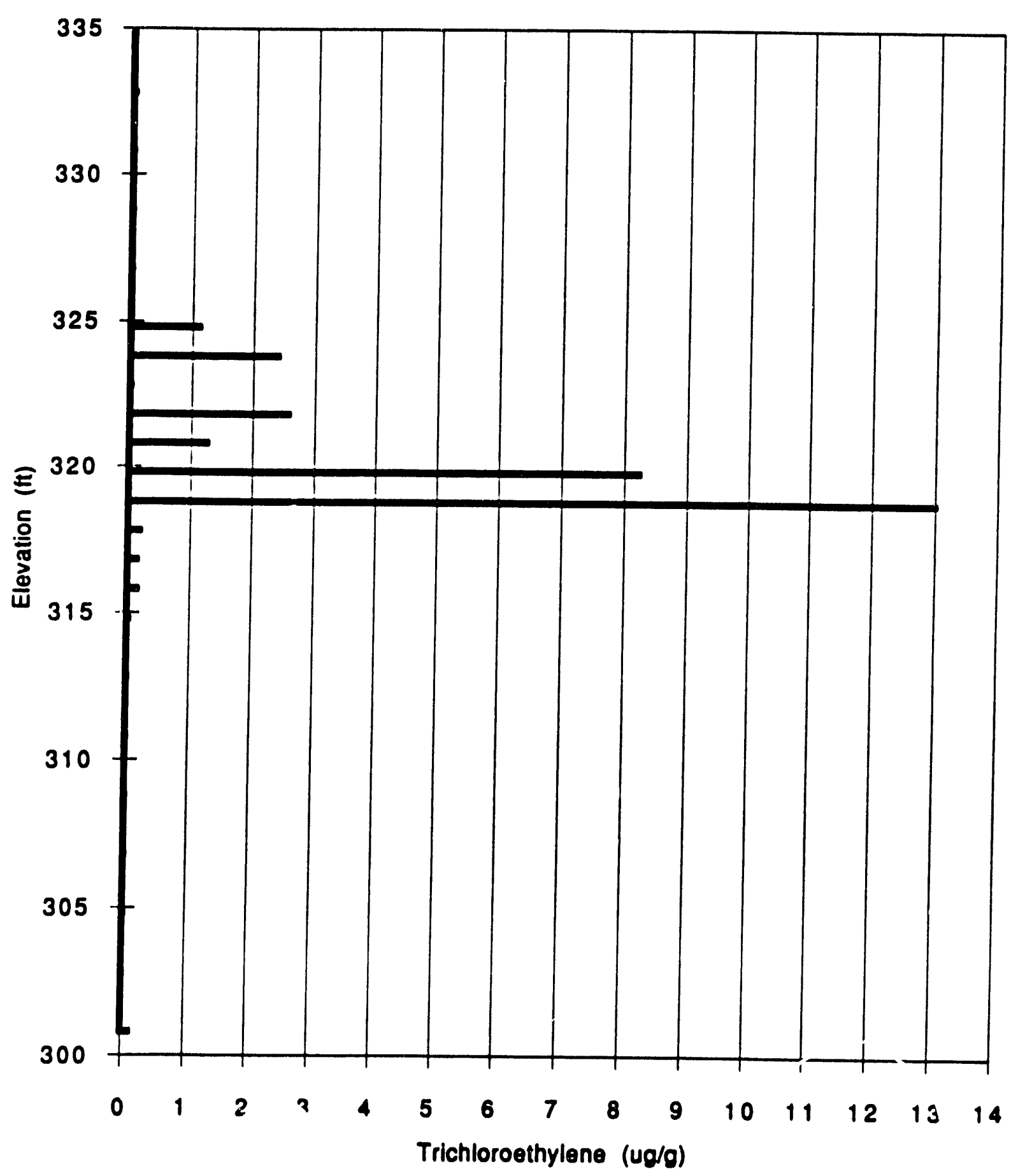




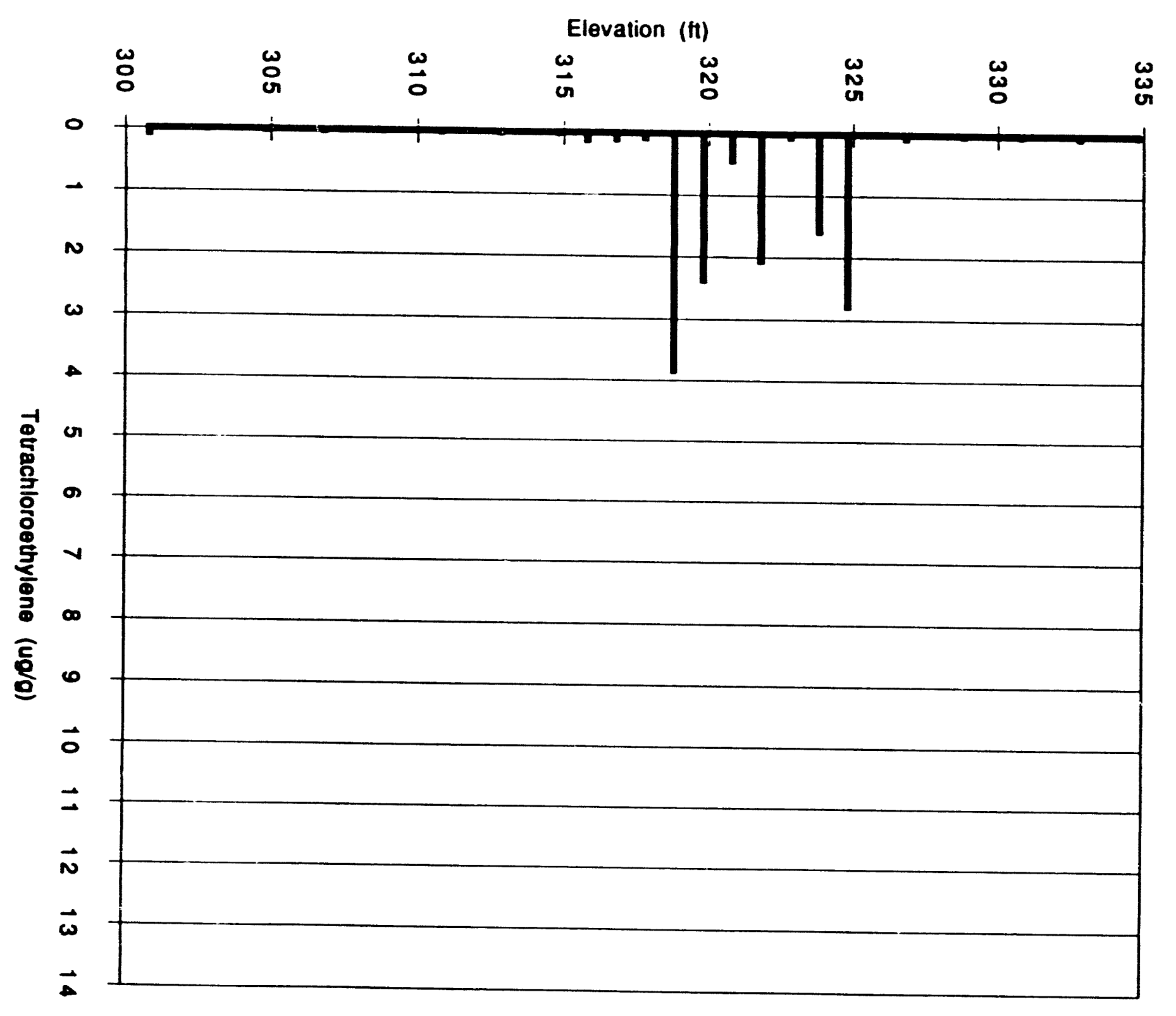

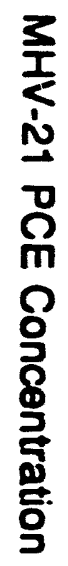




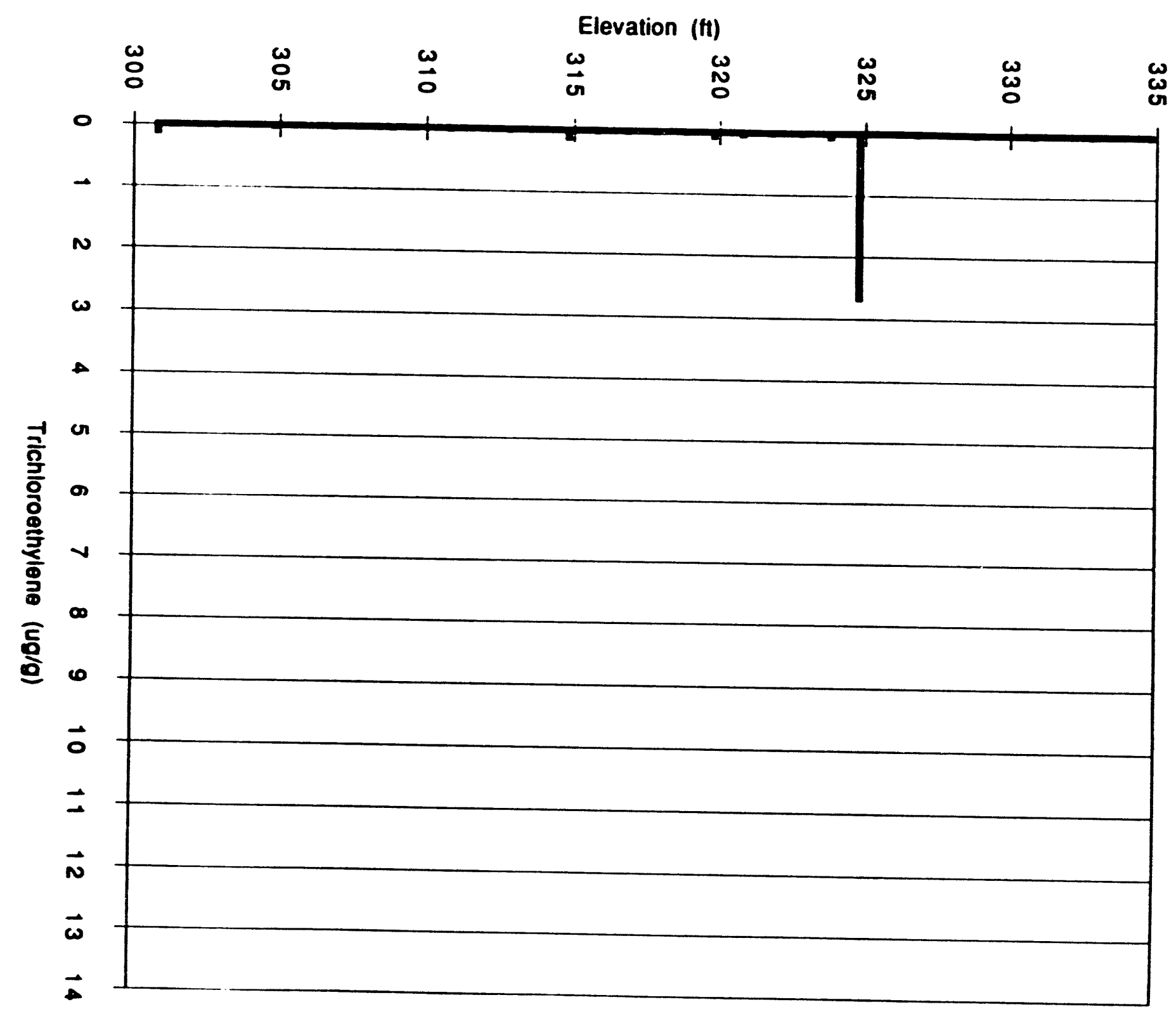




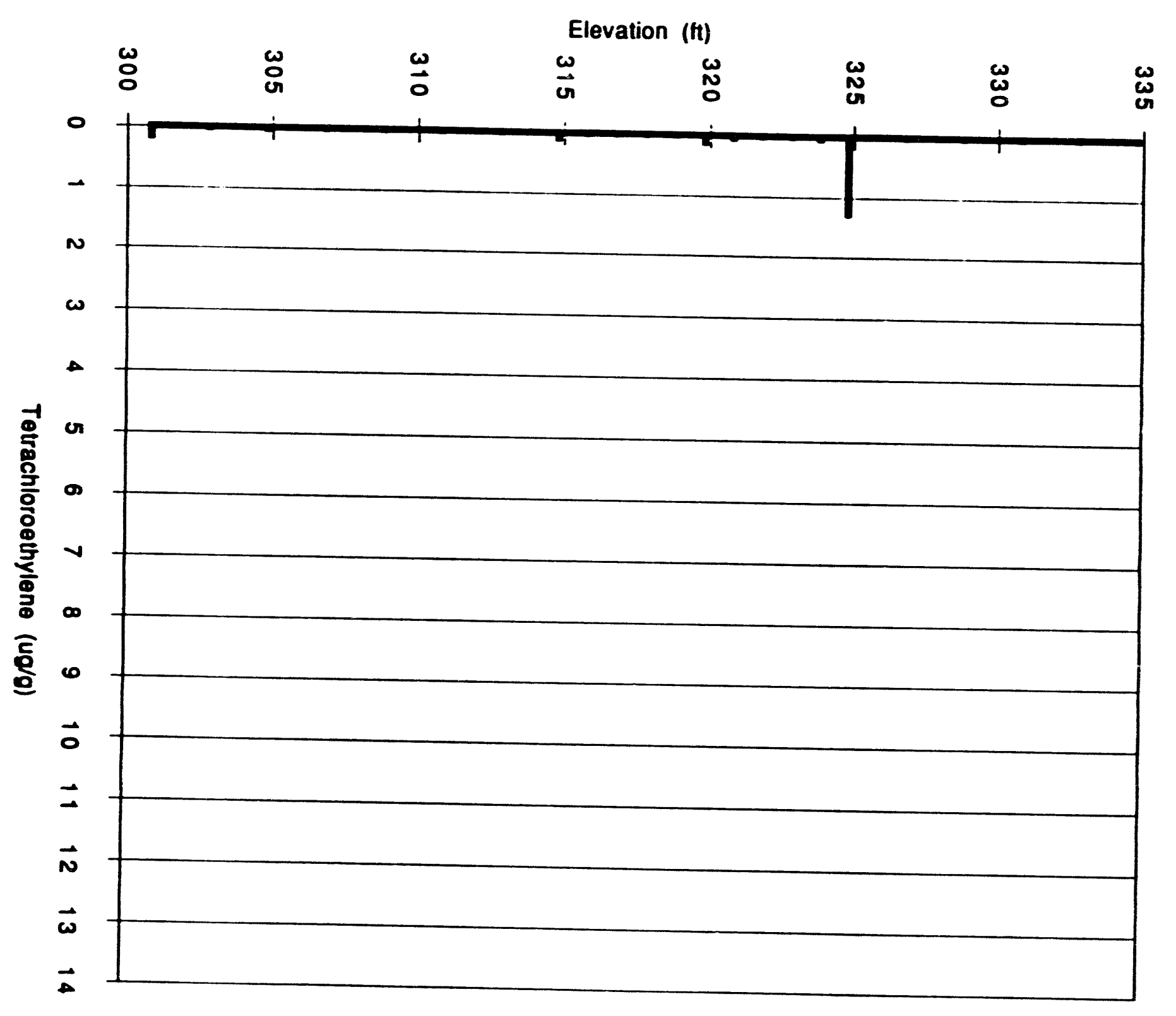


MHV-23 TCE Concentration

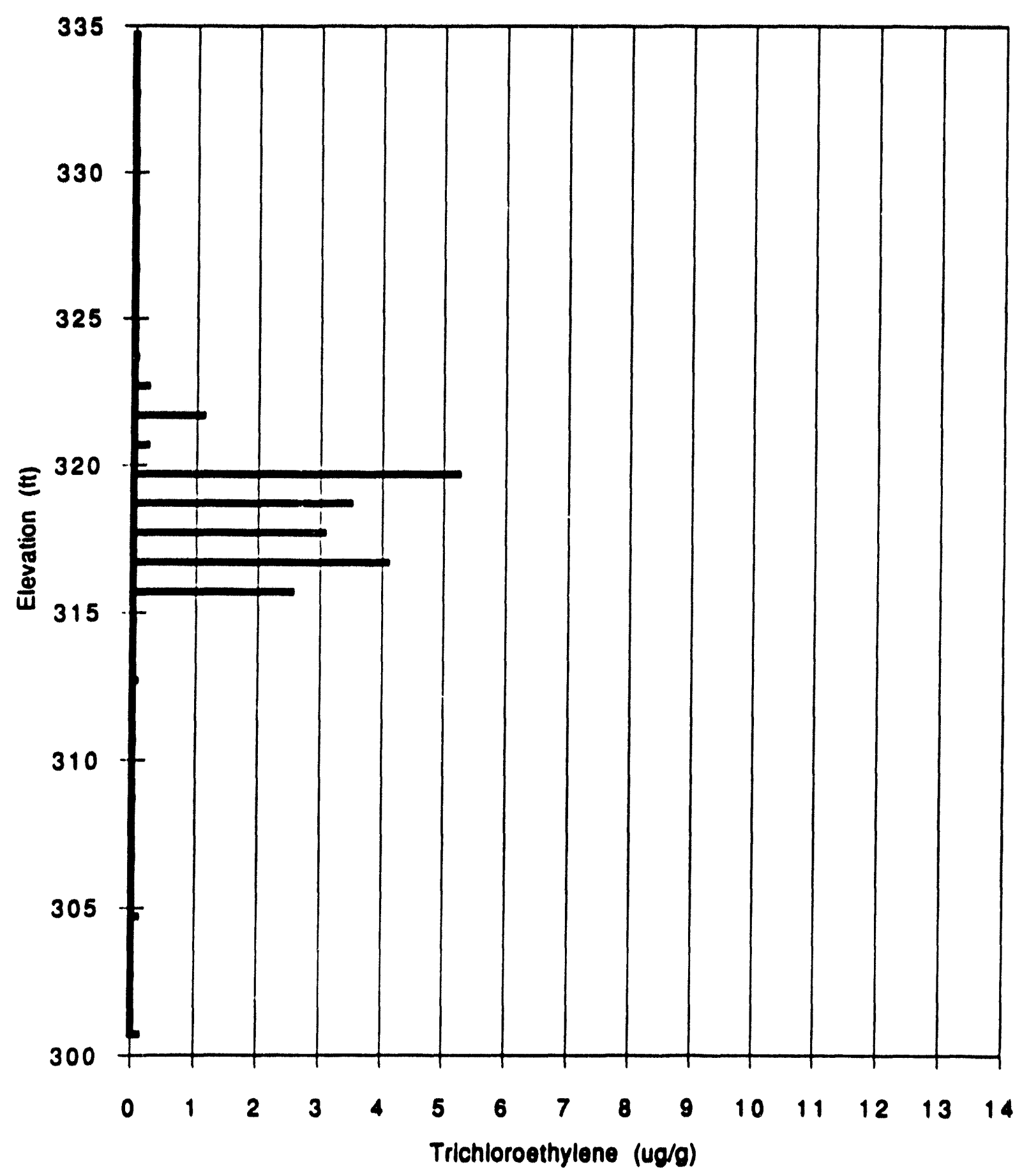


MHV-23 PCE Concentration

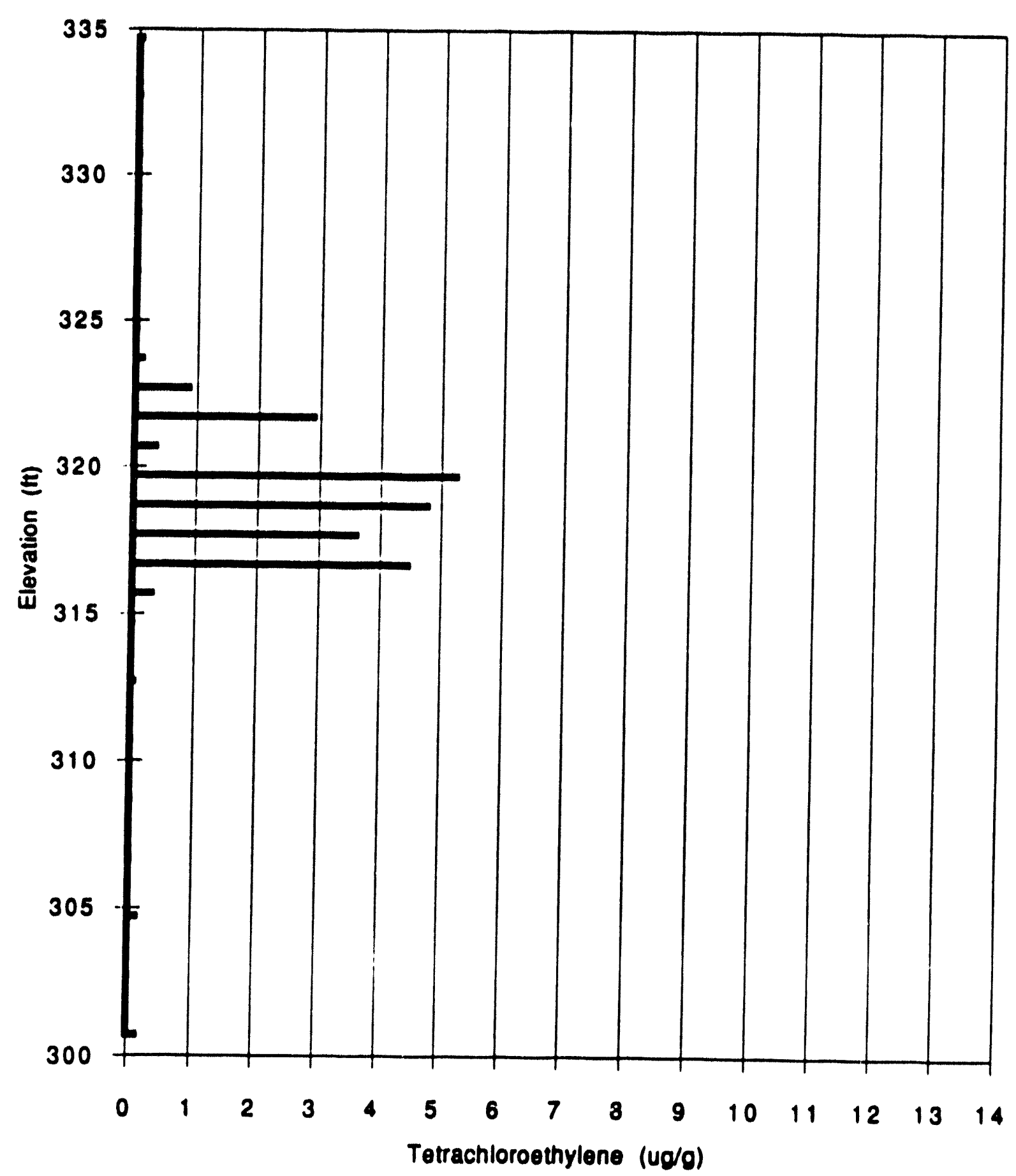




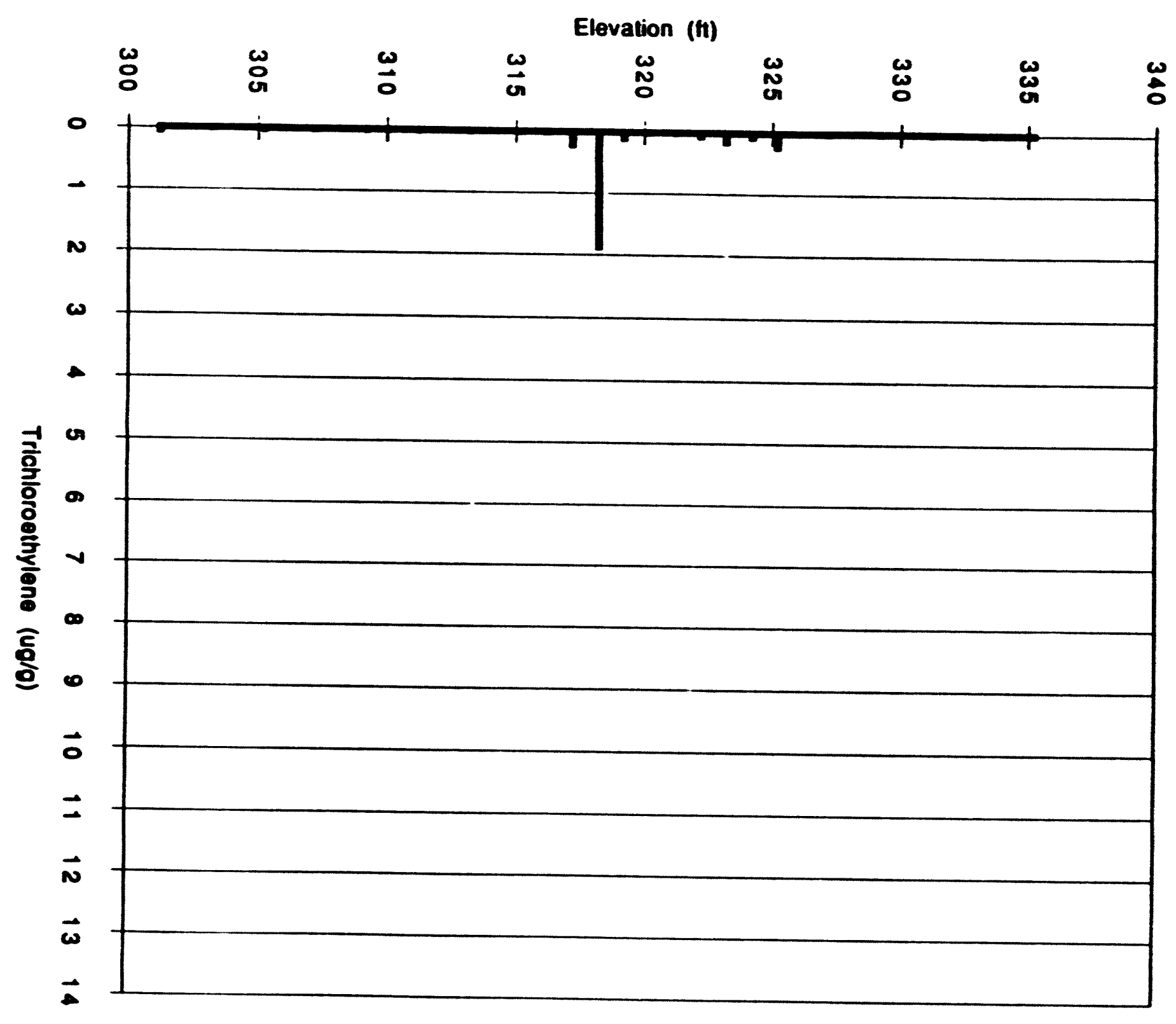


MHV-24 PCE Concentration

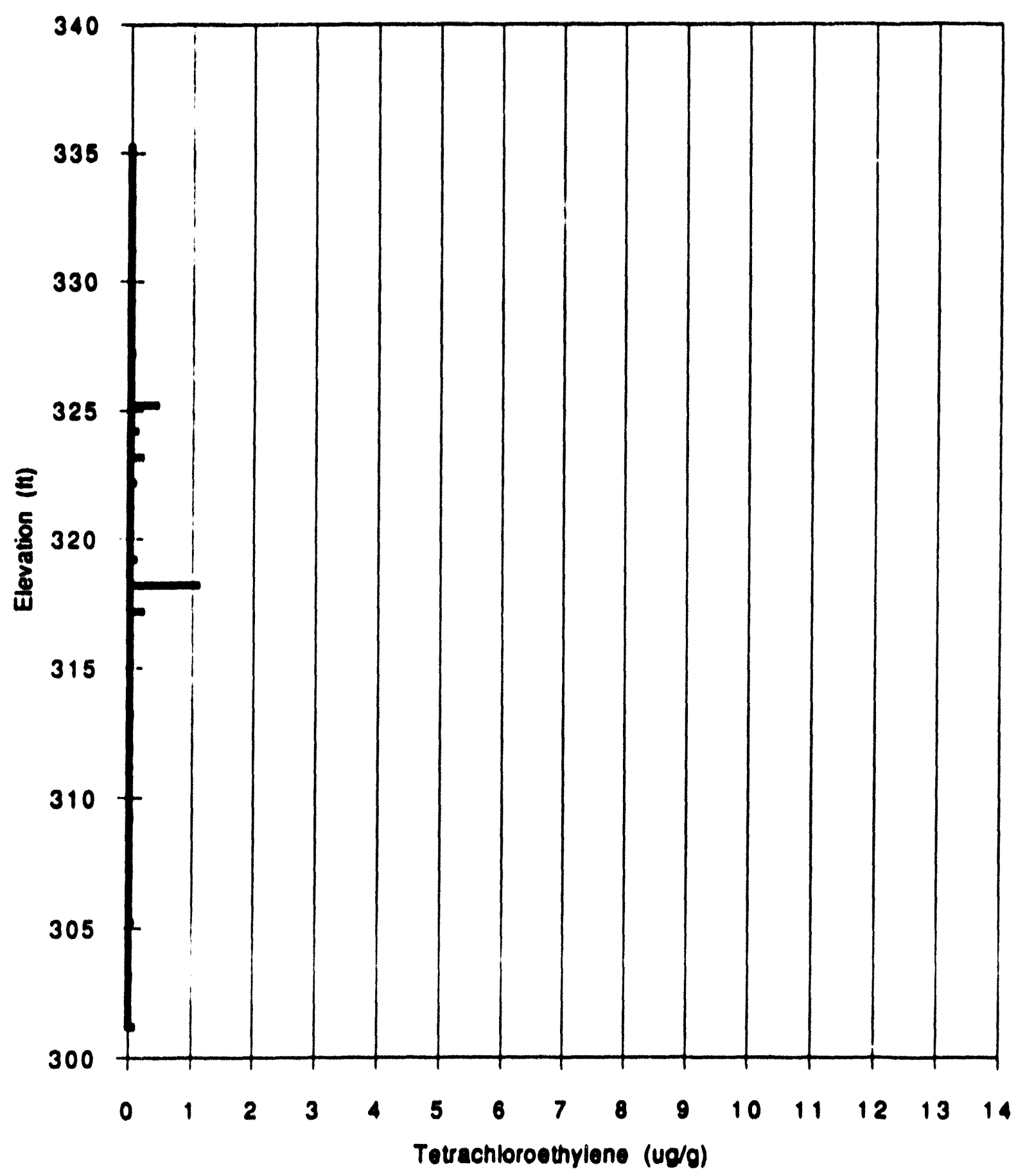




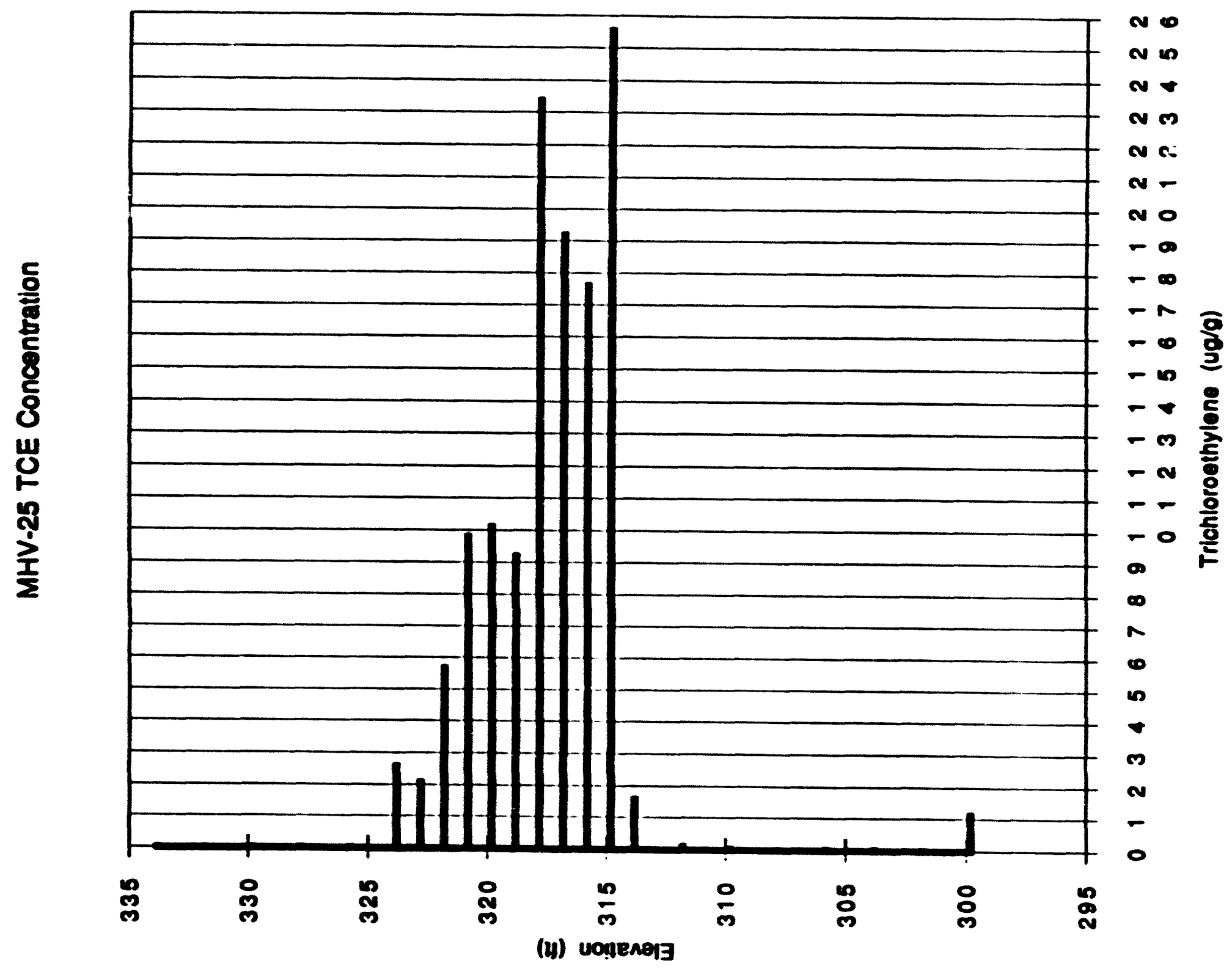


MHV-25 PCE Concentration

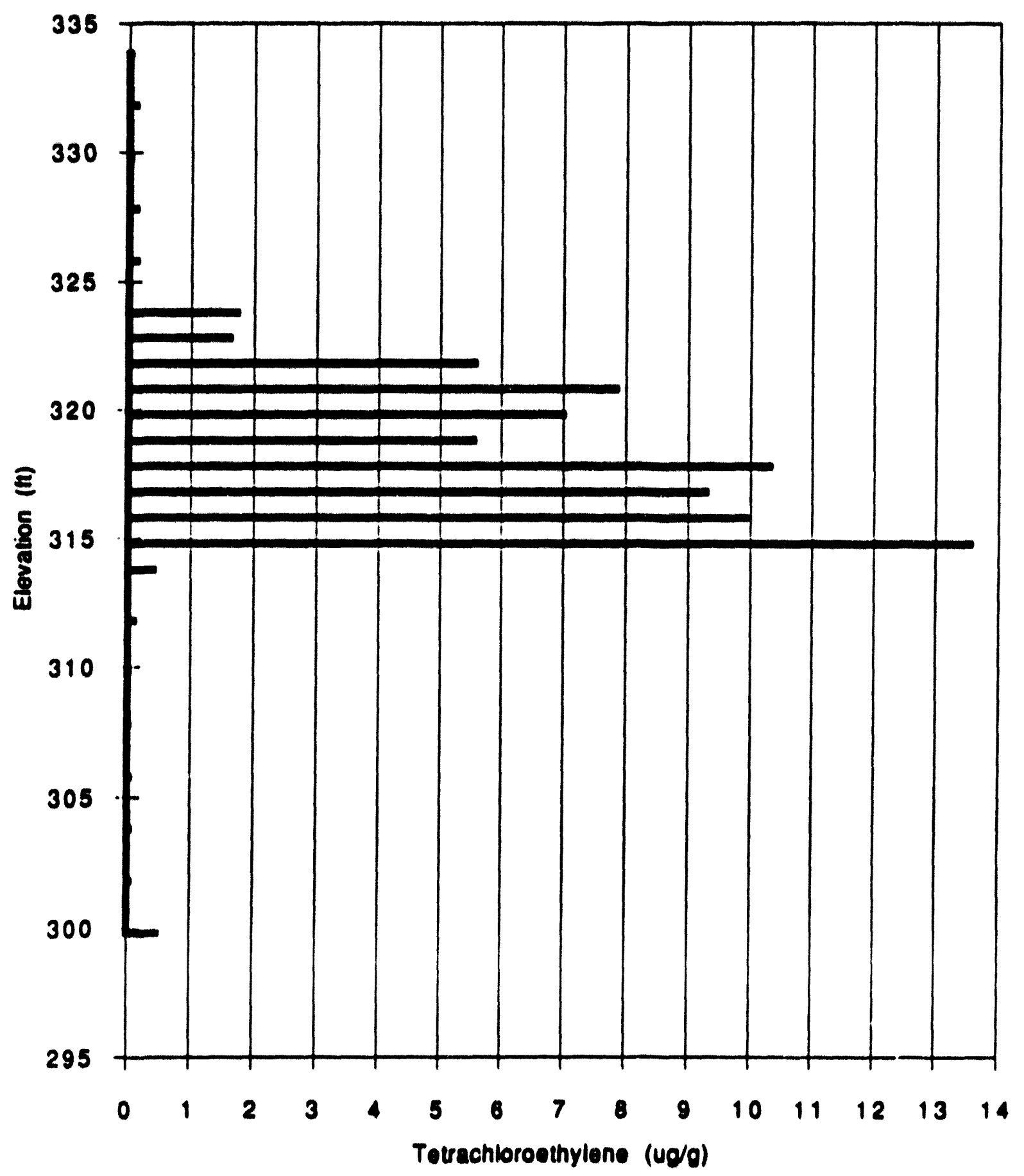


MHV-26 TCE Concentration

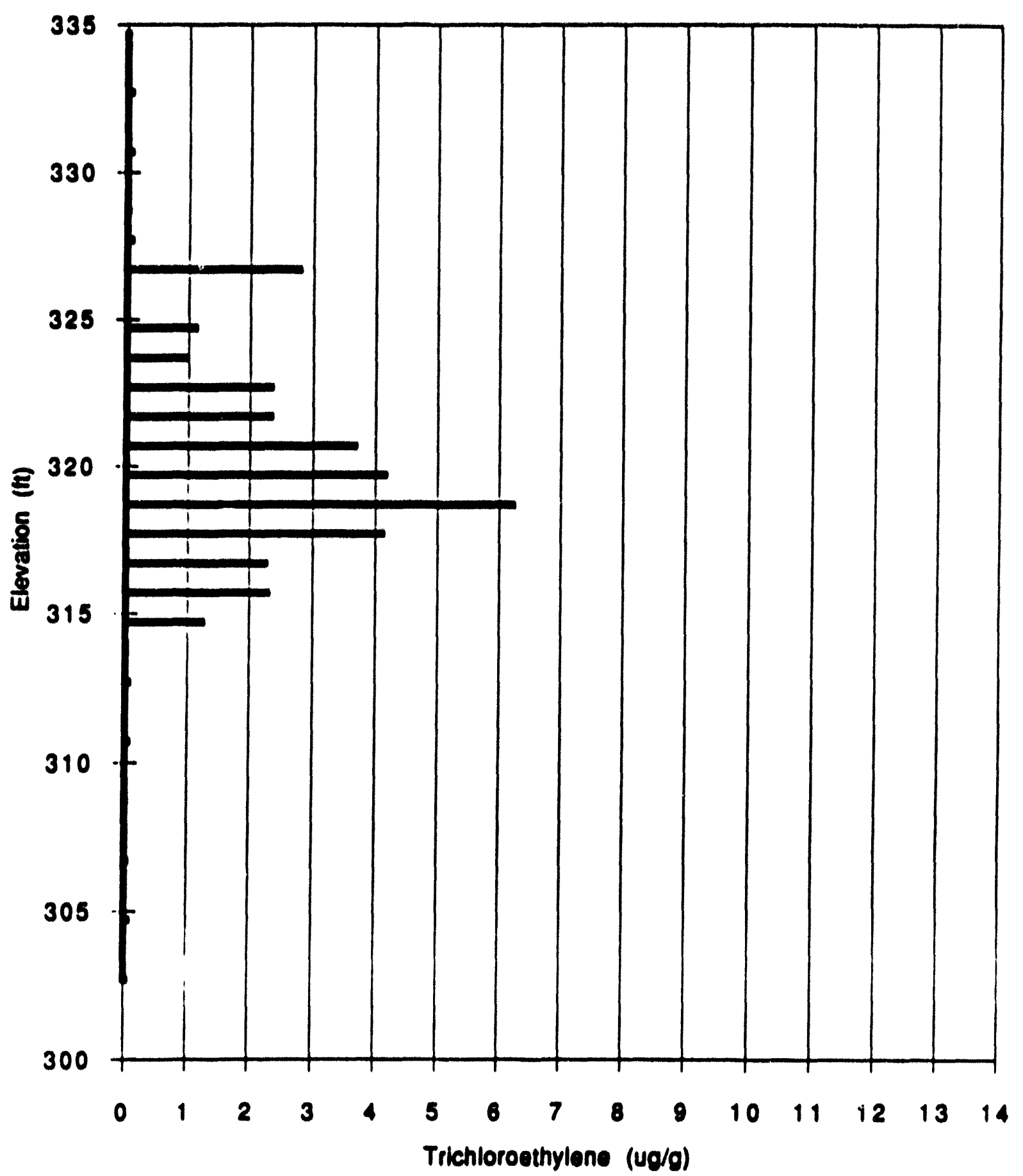


MHV-26 PCE Concentration

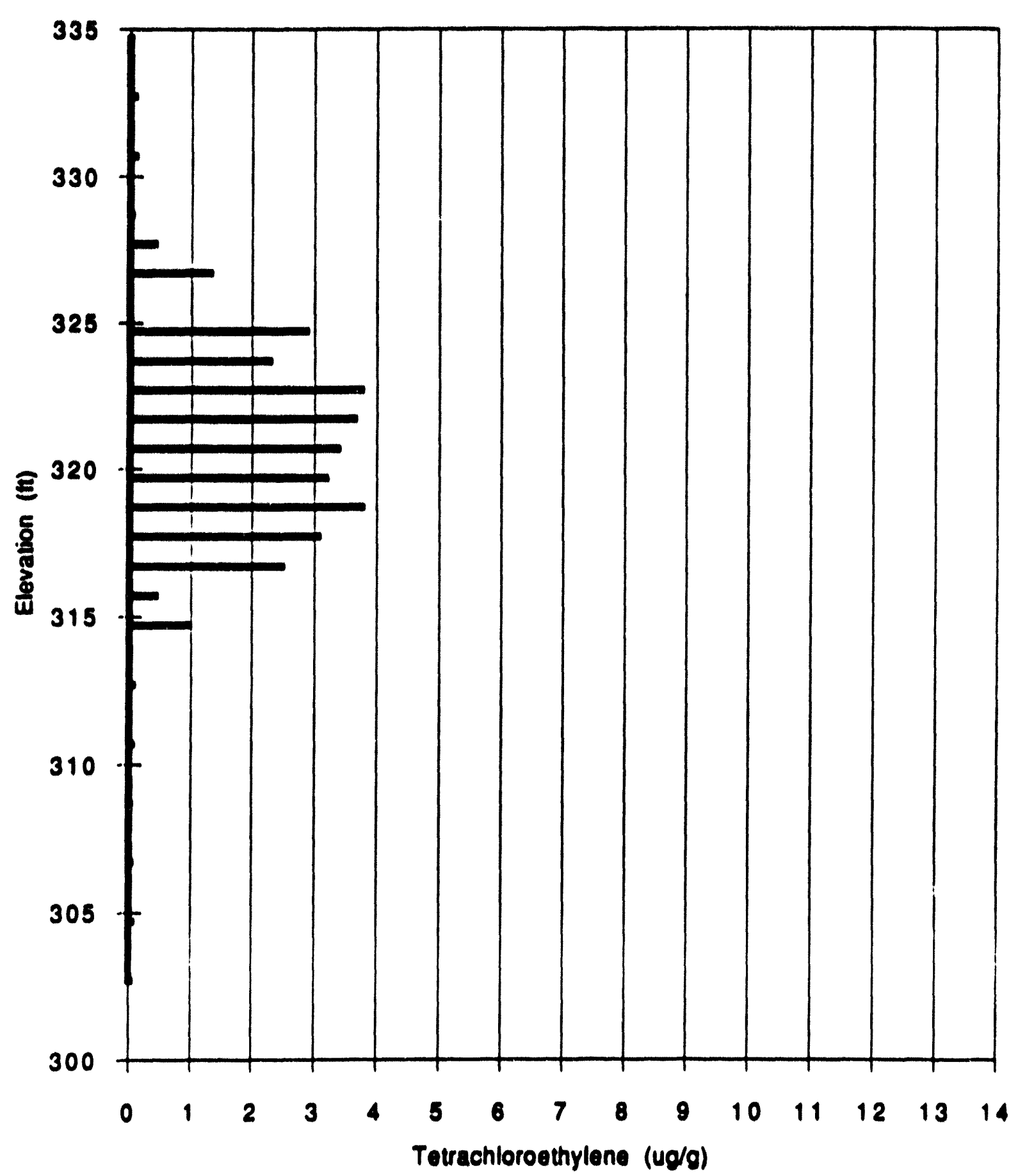




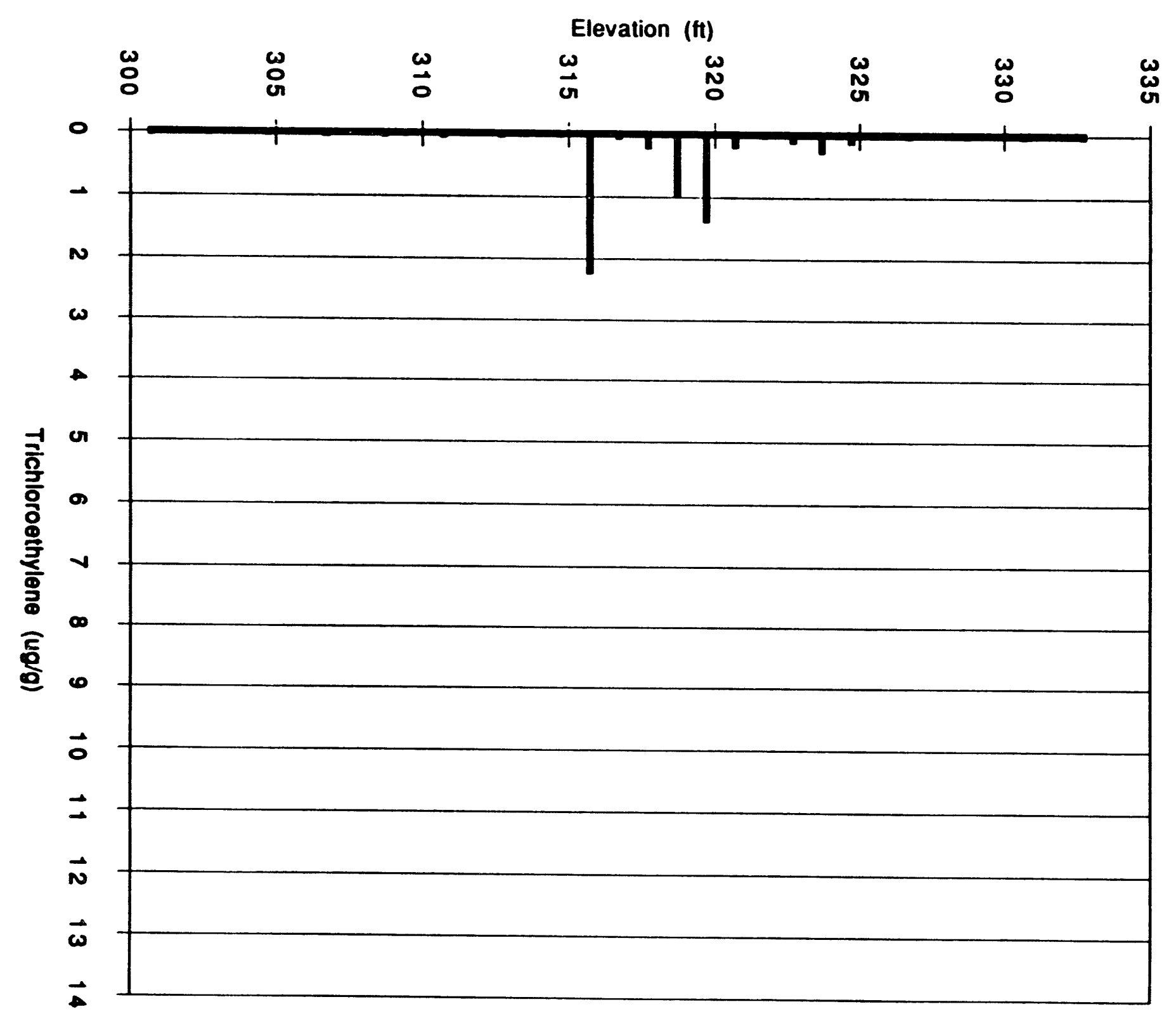




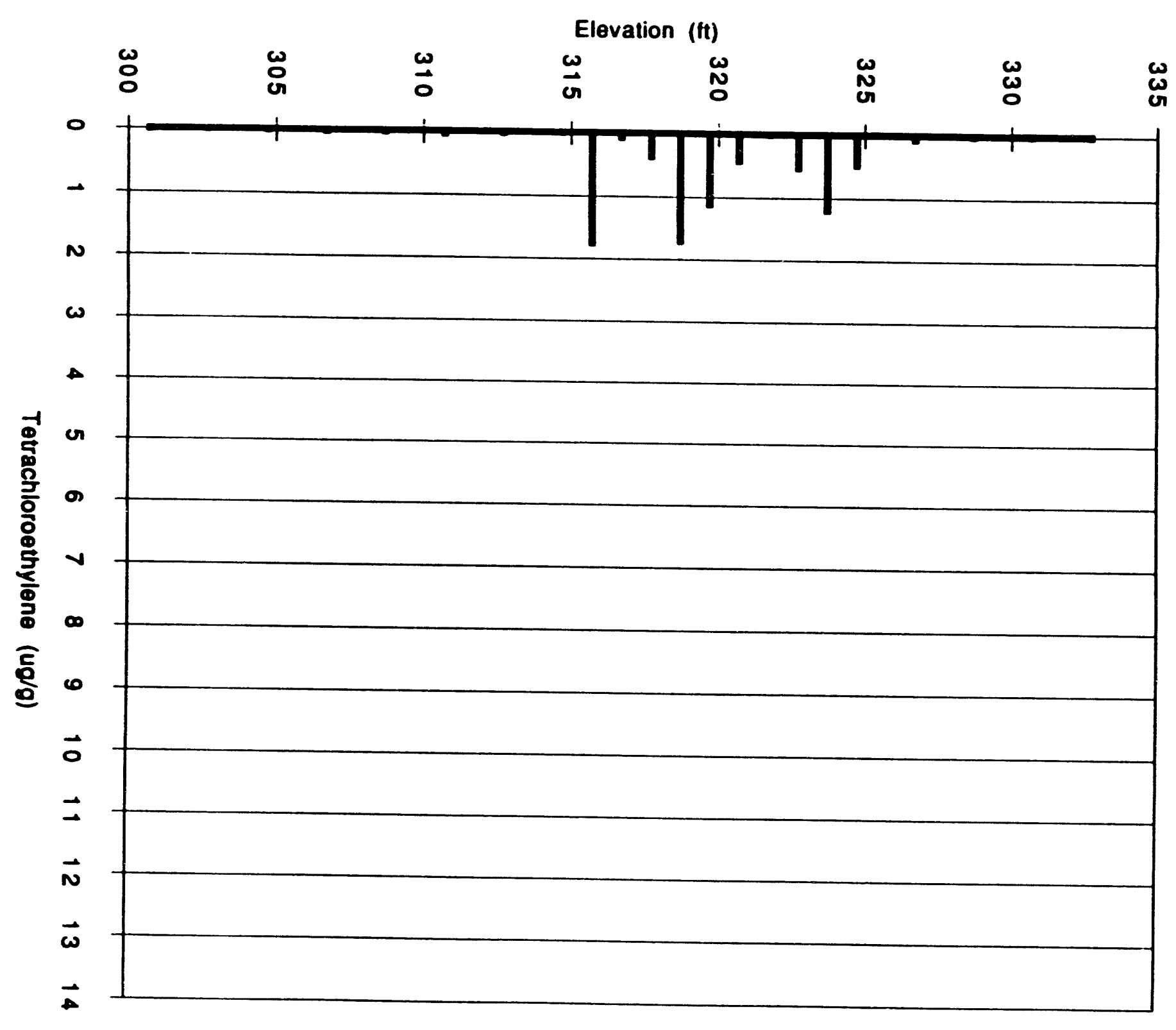




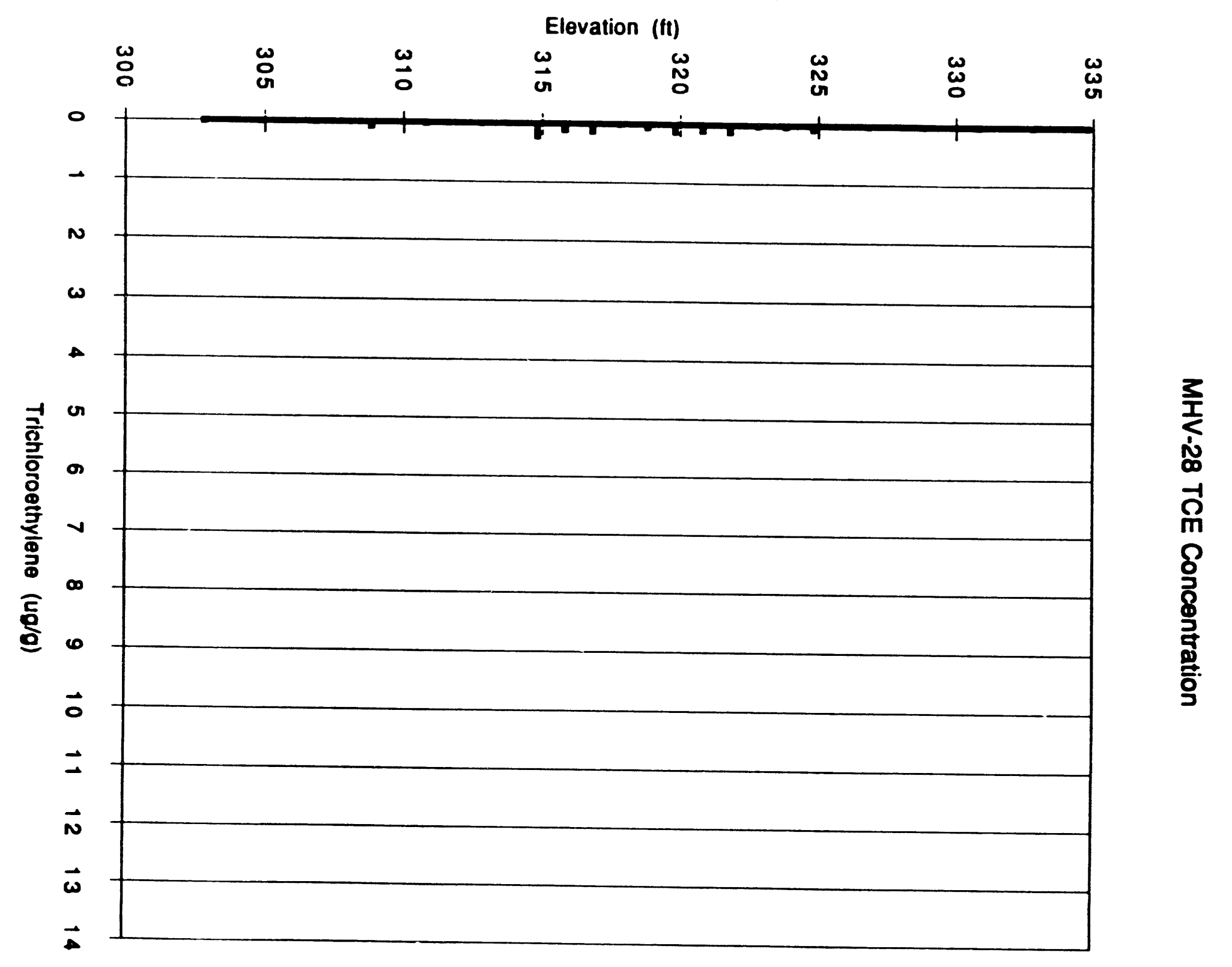




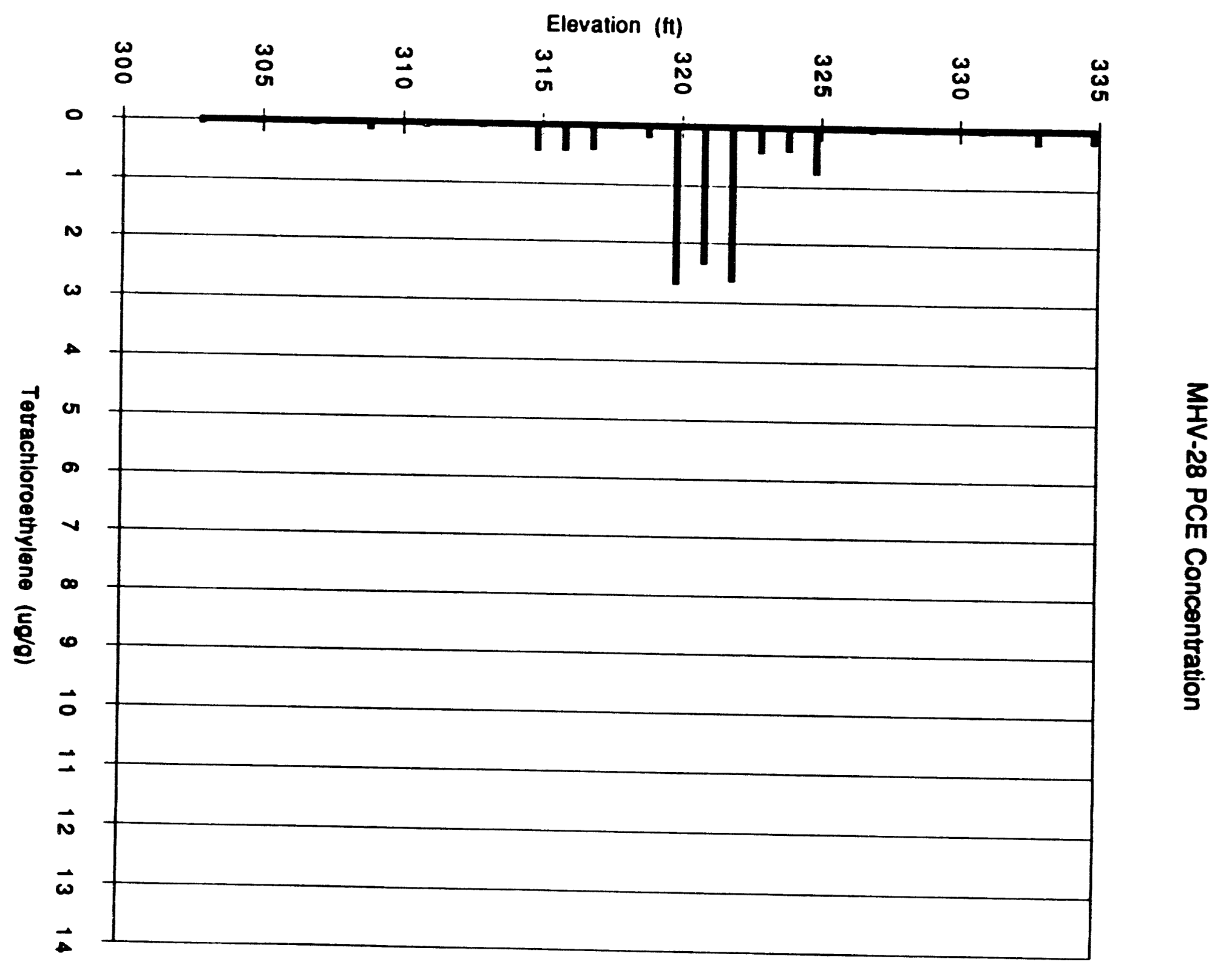




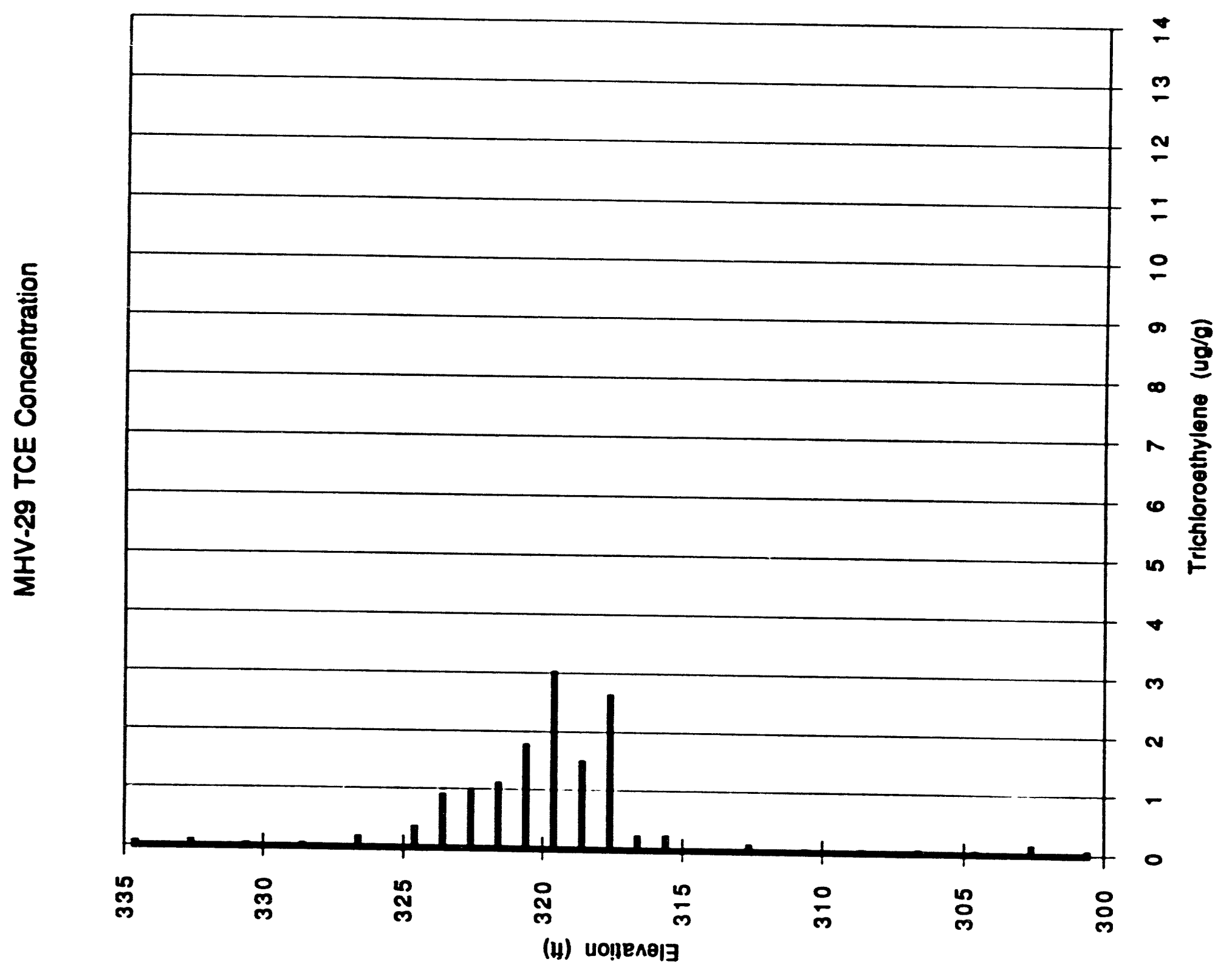




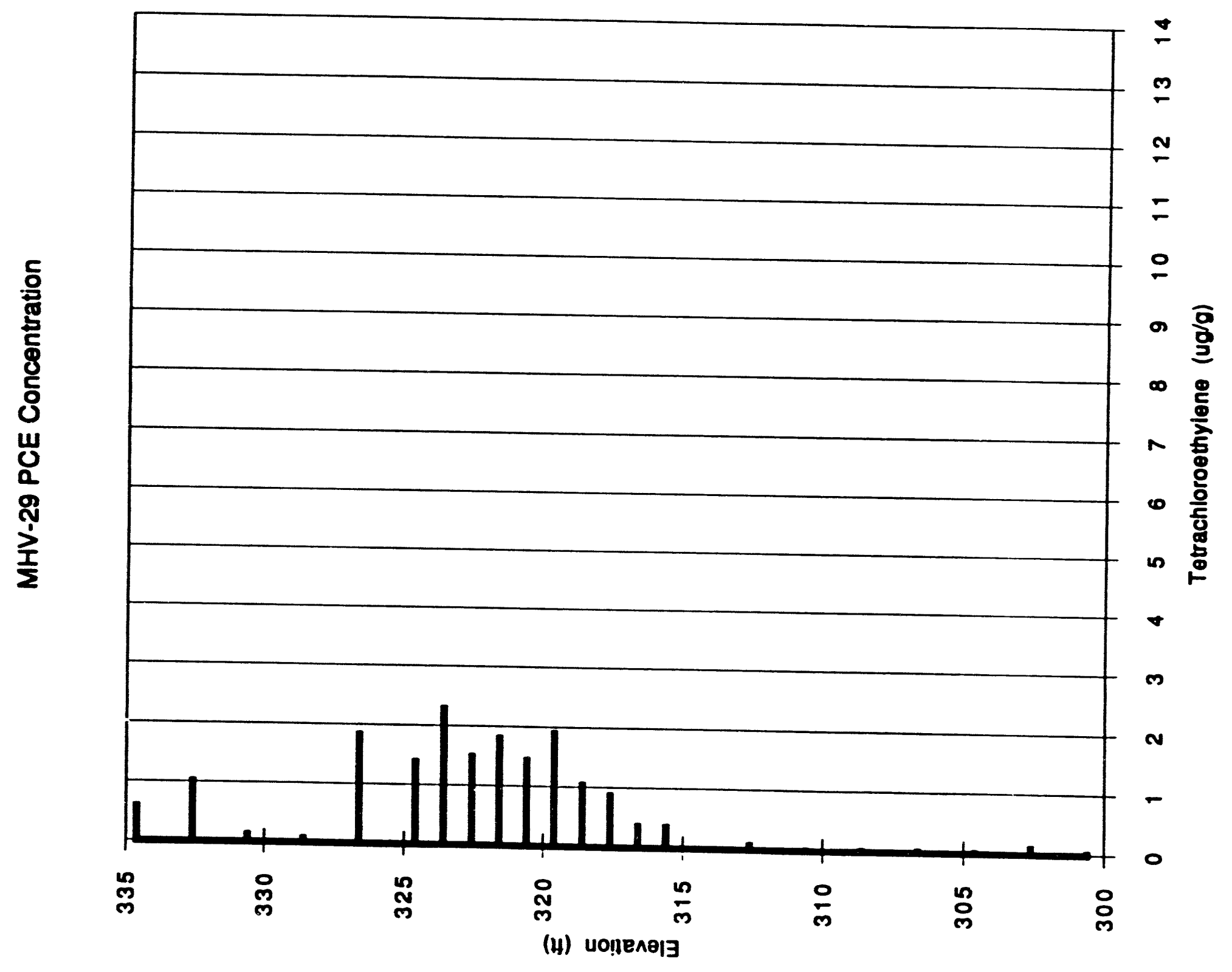


MHB-20 TCE Concentration

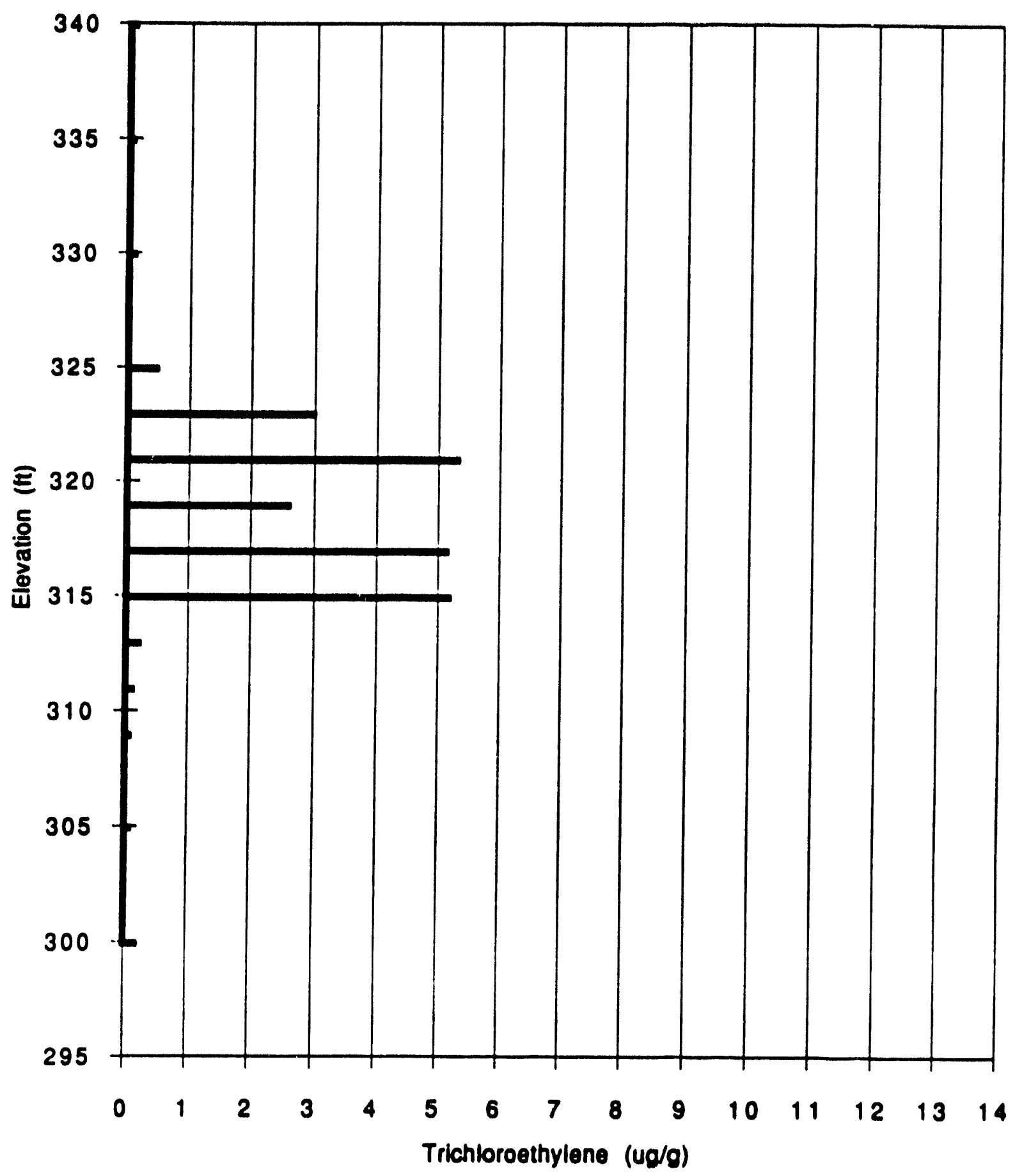


MHB-20 PCE Concentration

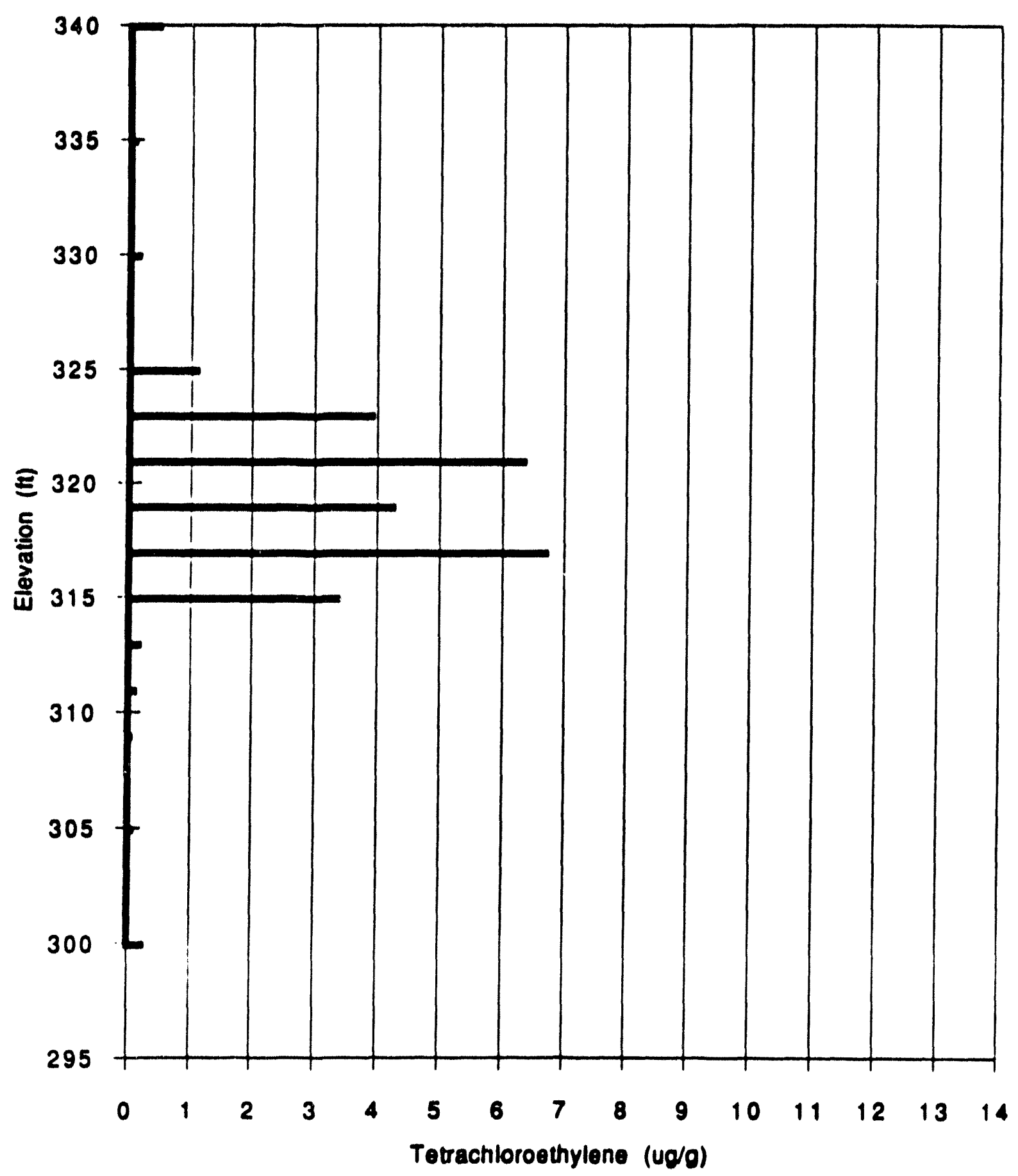




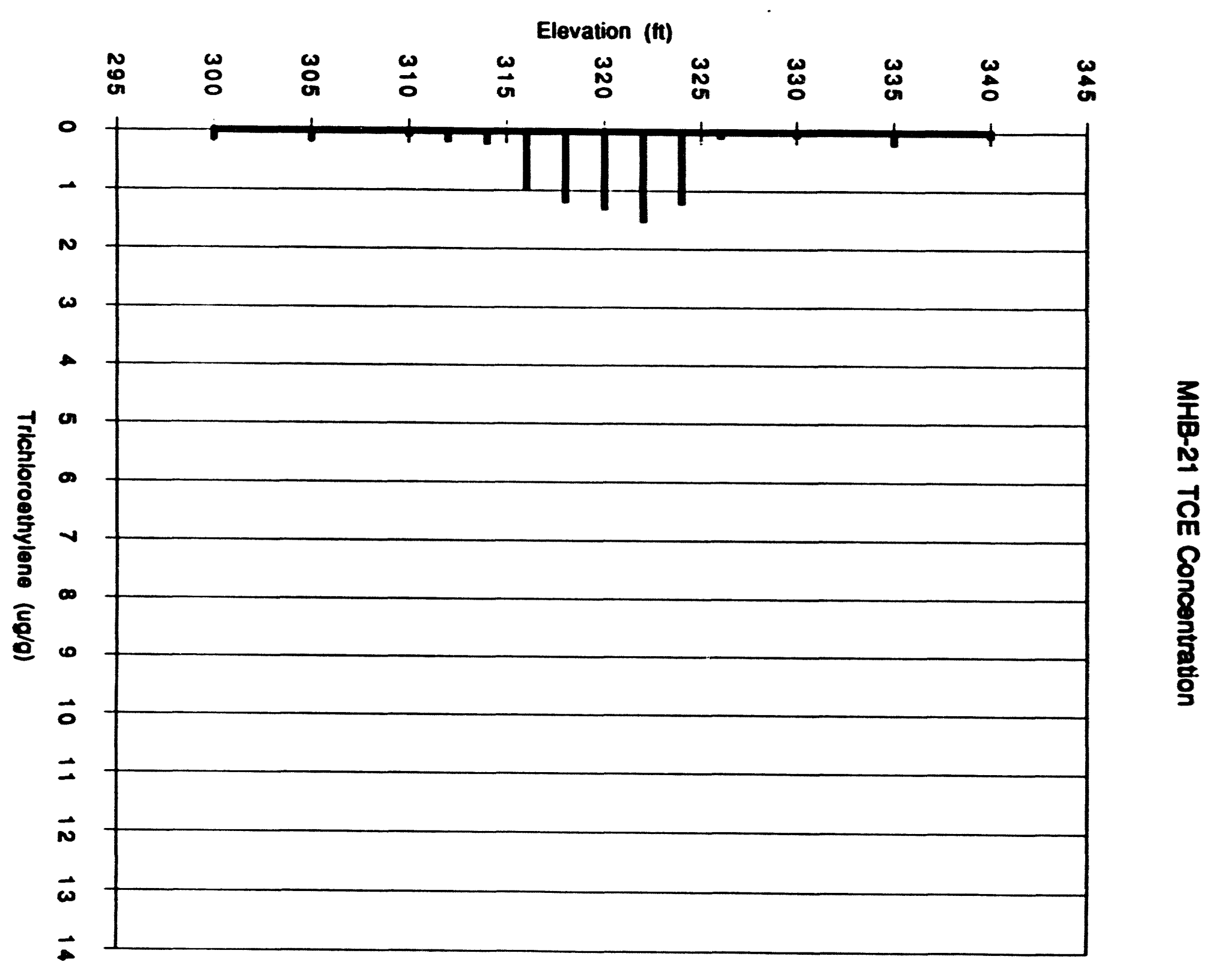


MHB-21 PCE Concentration

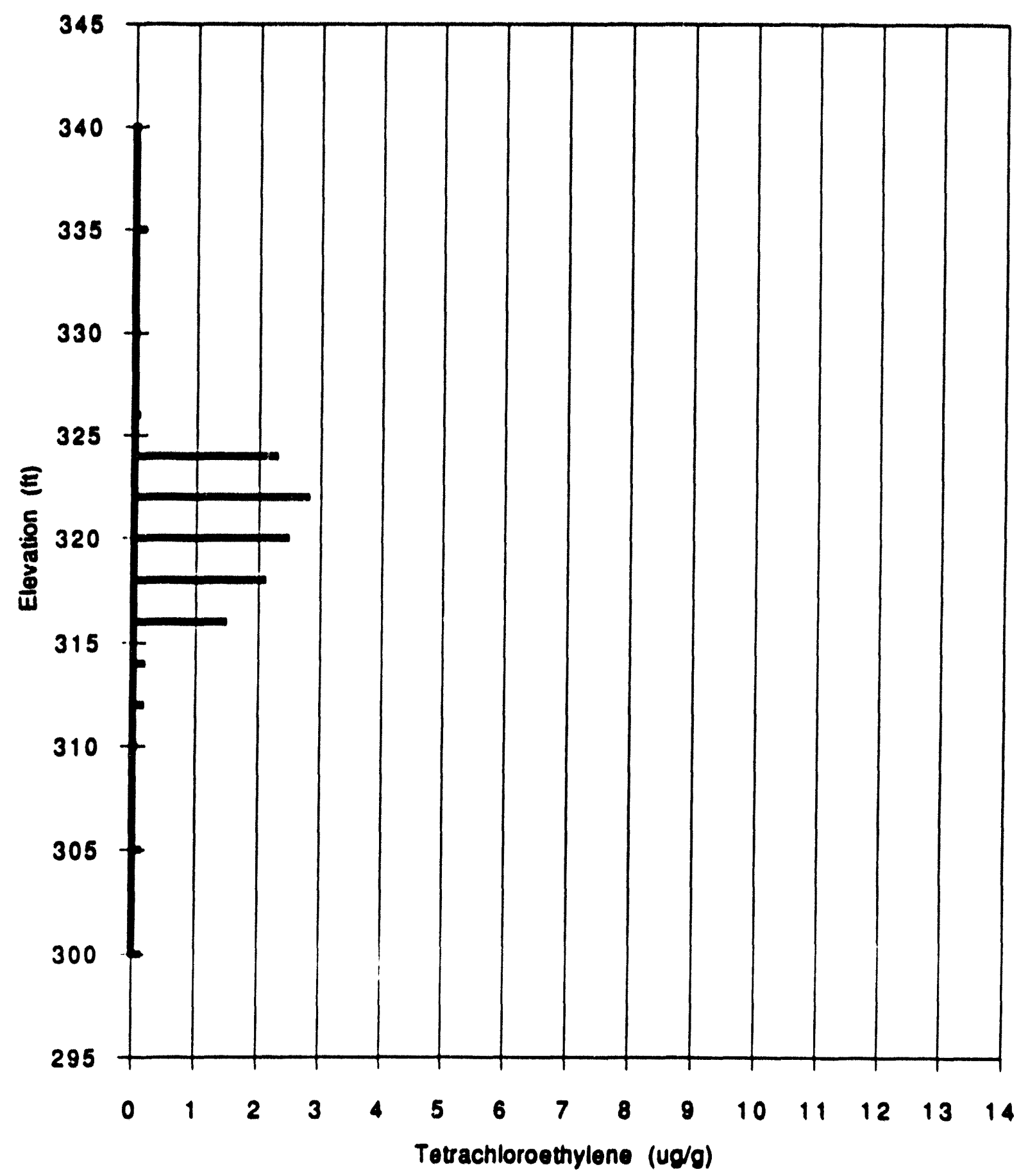




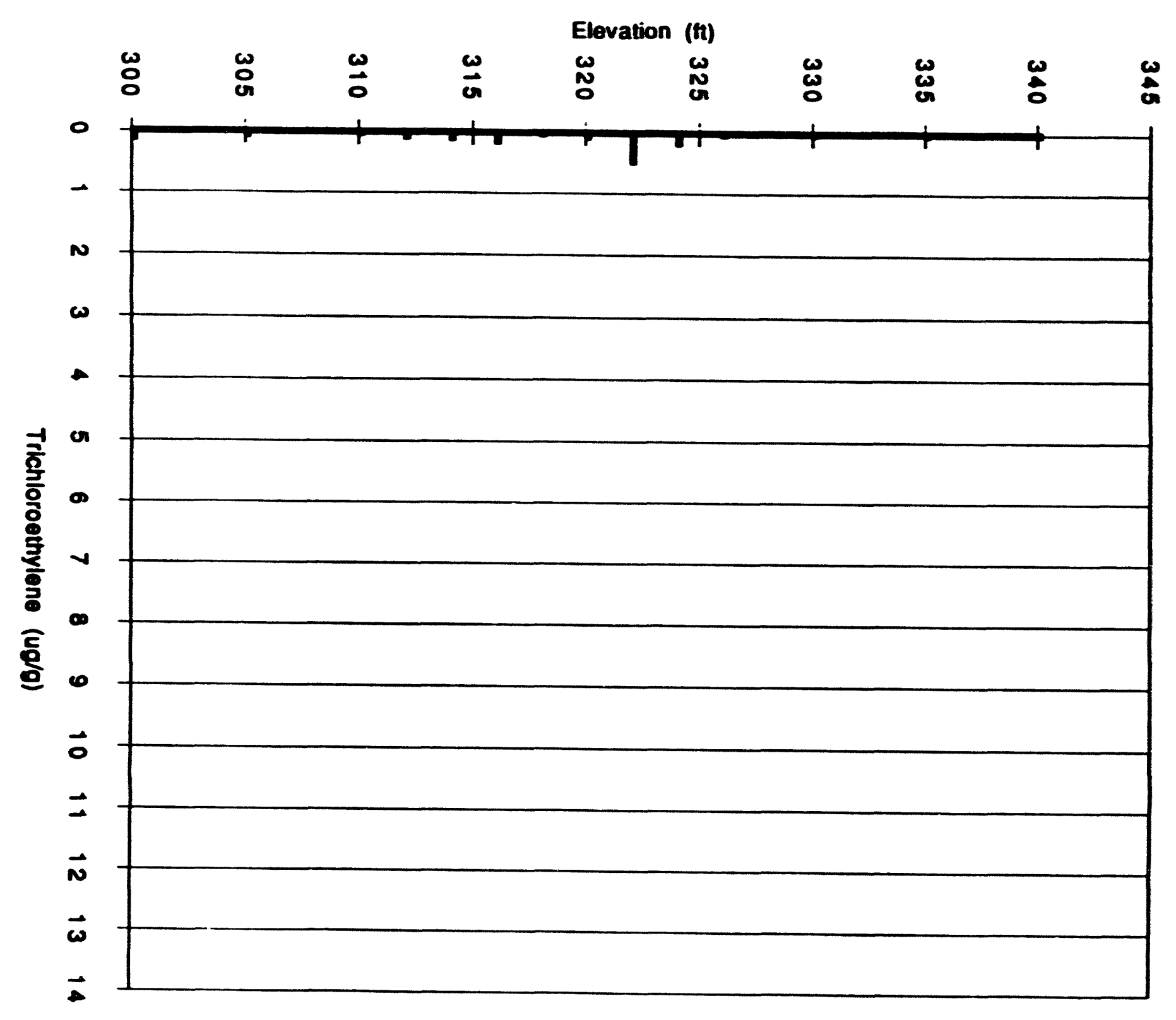




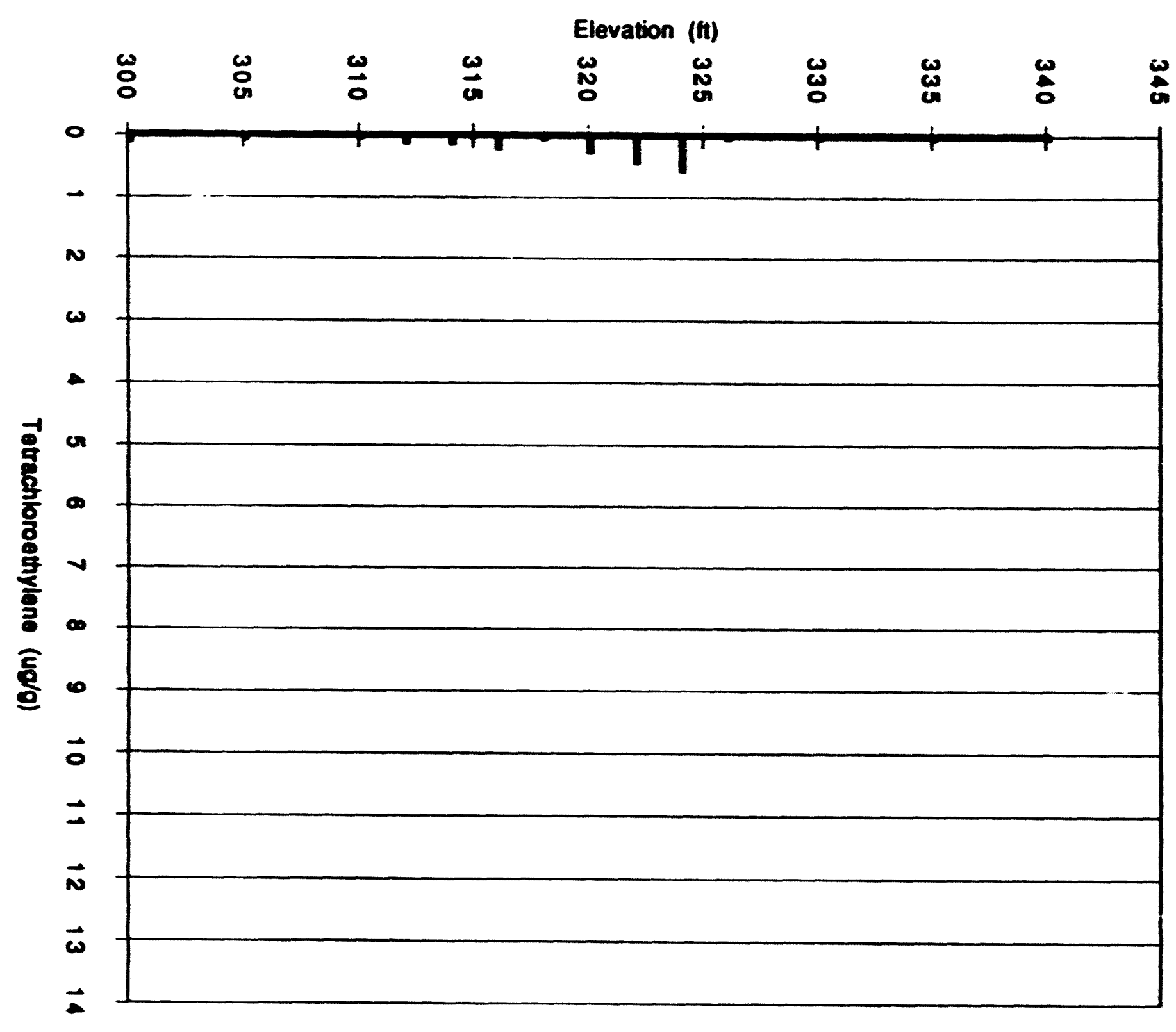


MHB-23 TCE Concentration

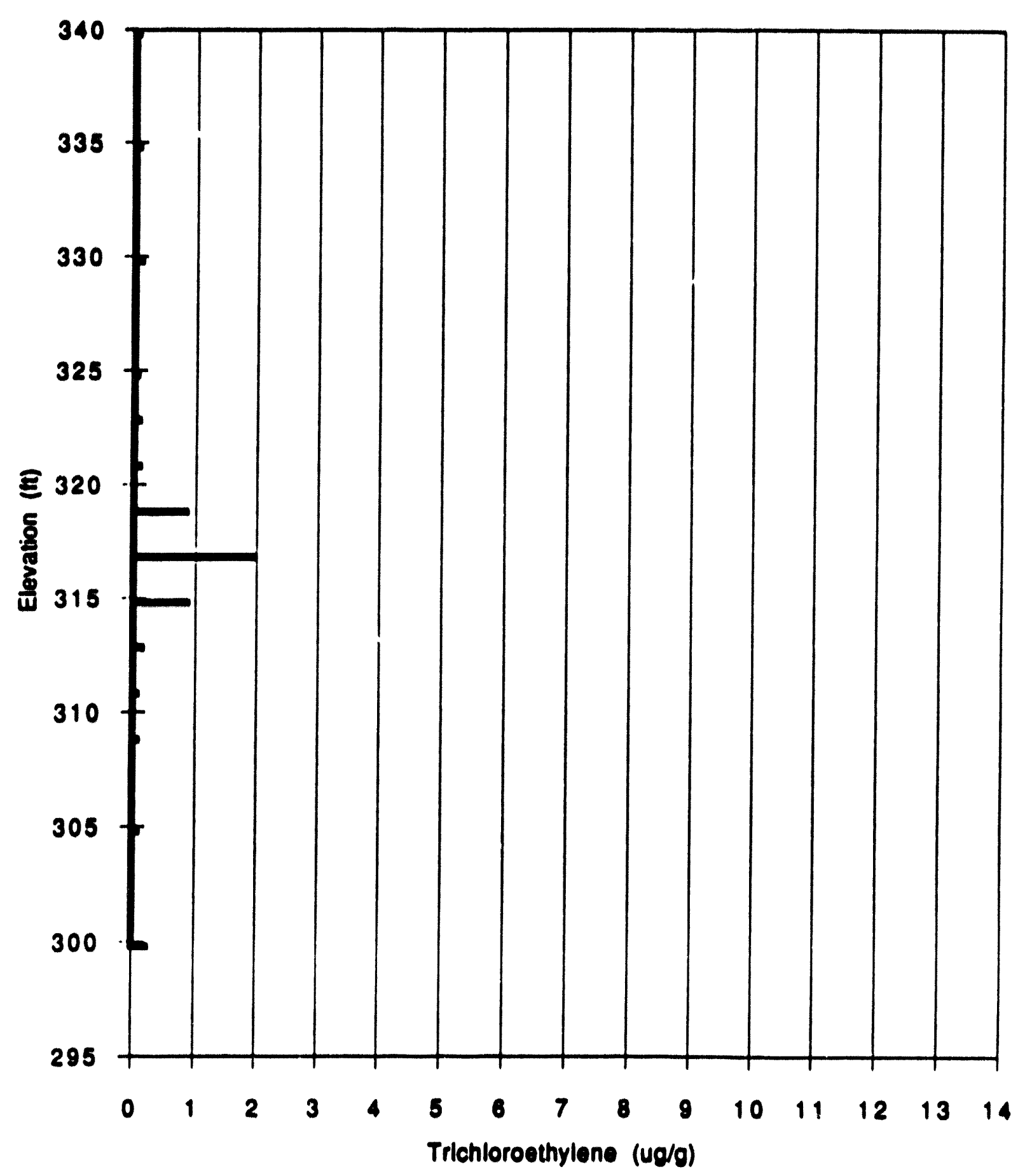


MHB-23 PCE Concentration

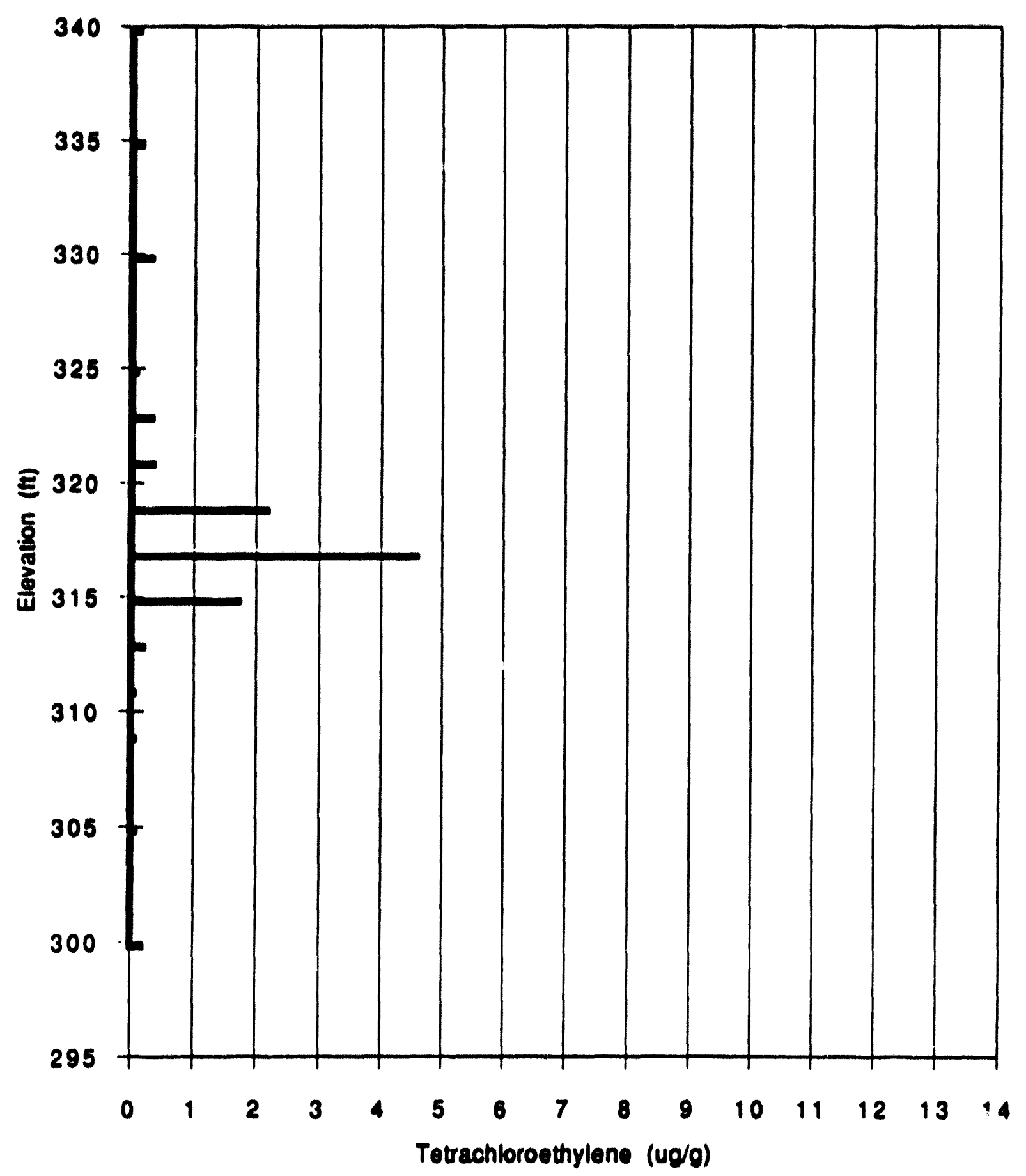




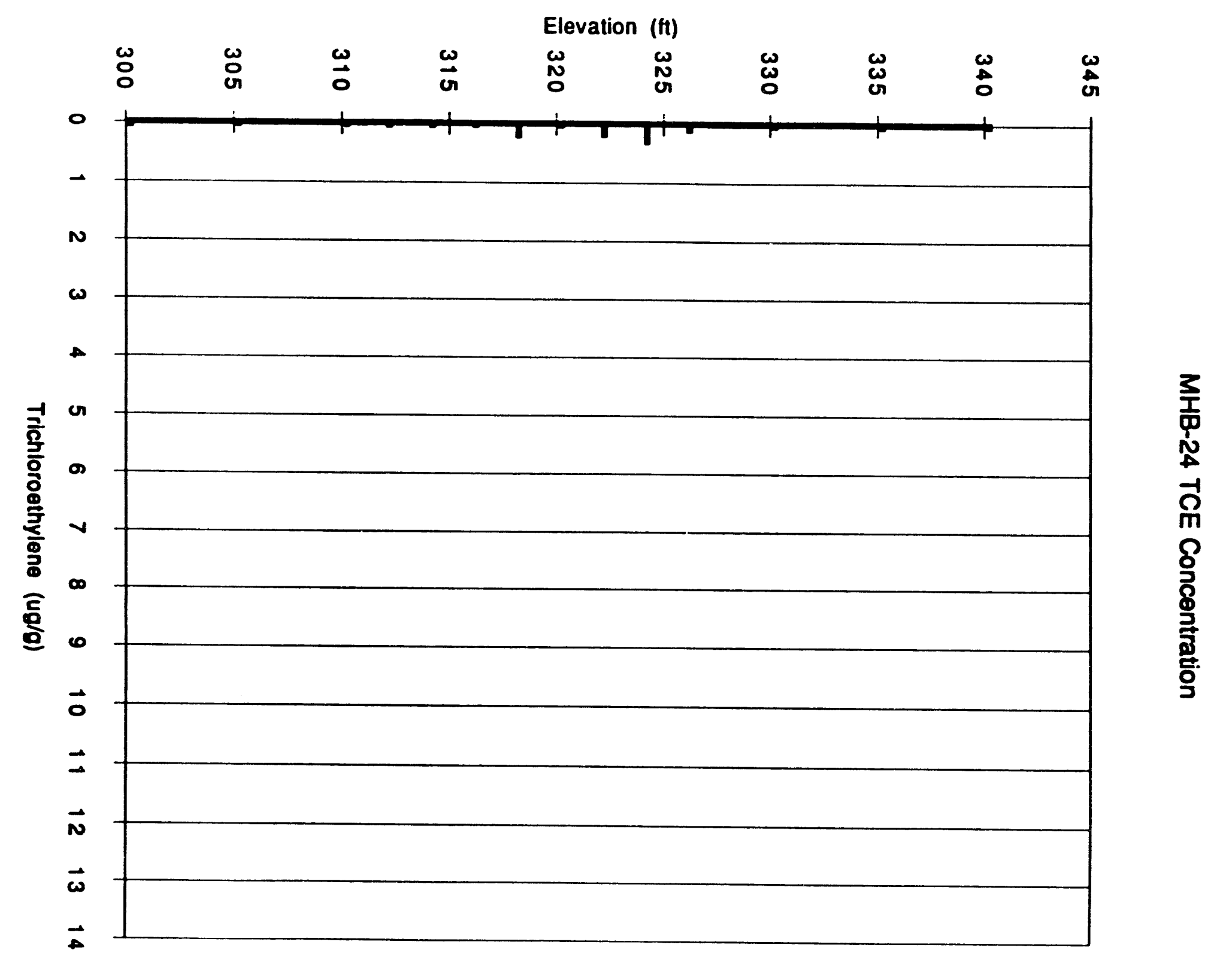


MHB-24 PCE Concentration

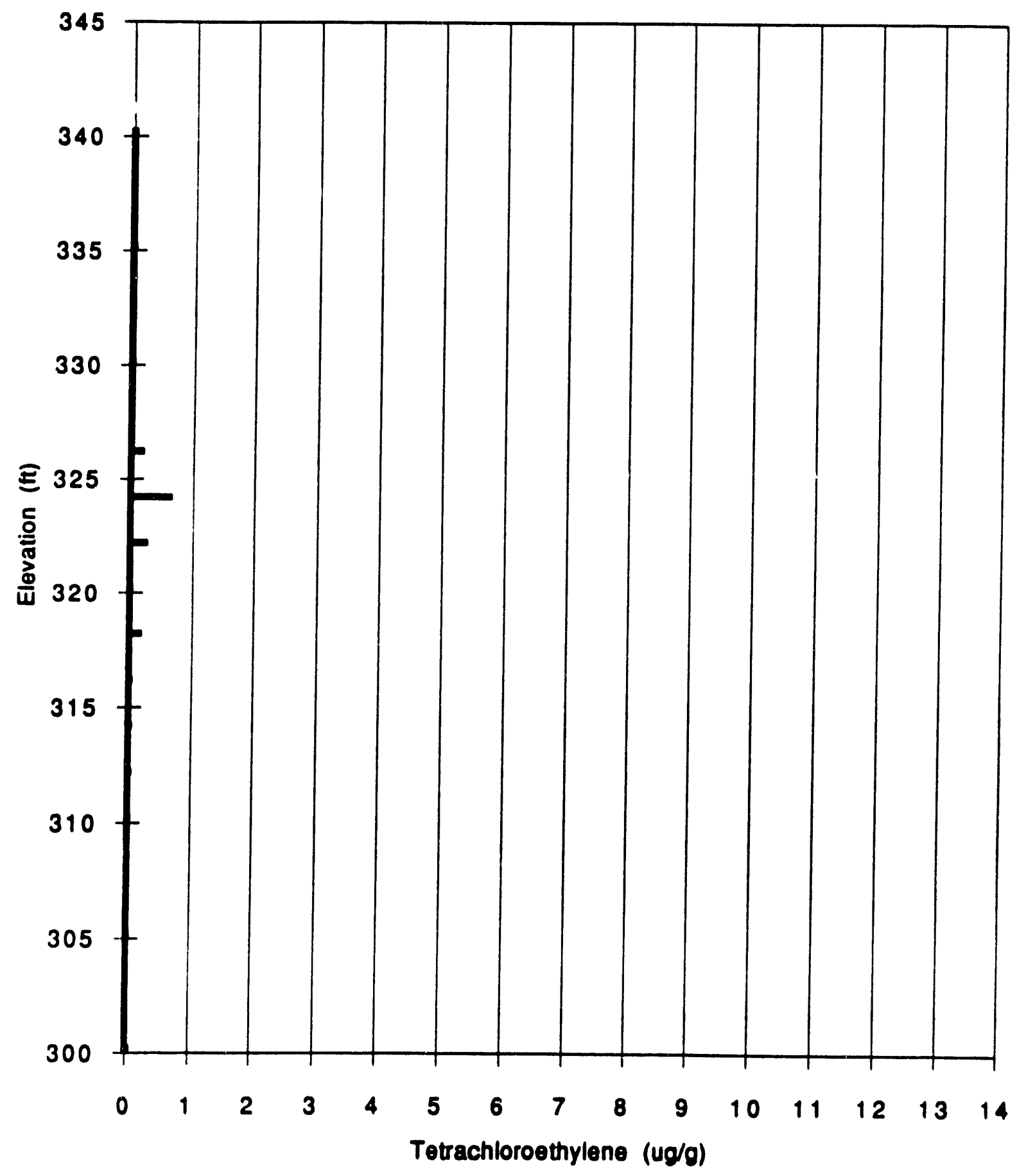


MHB-25 TCE Concentration

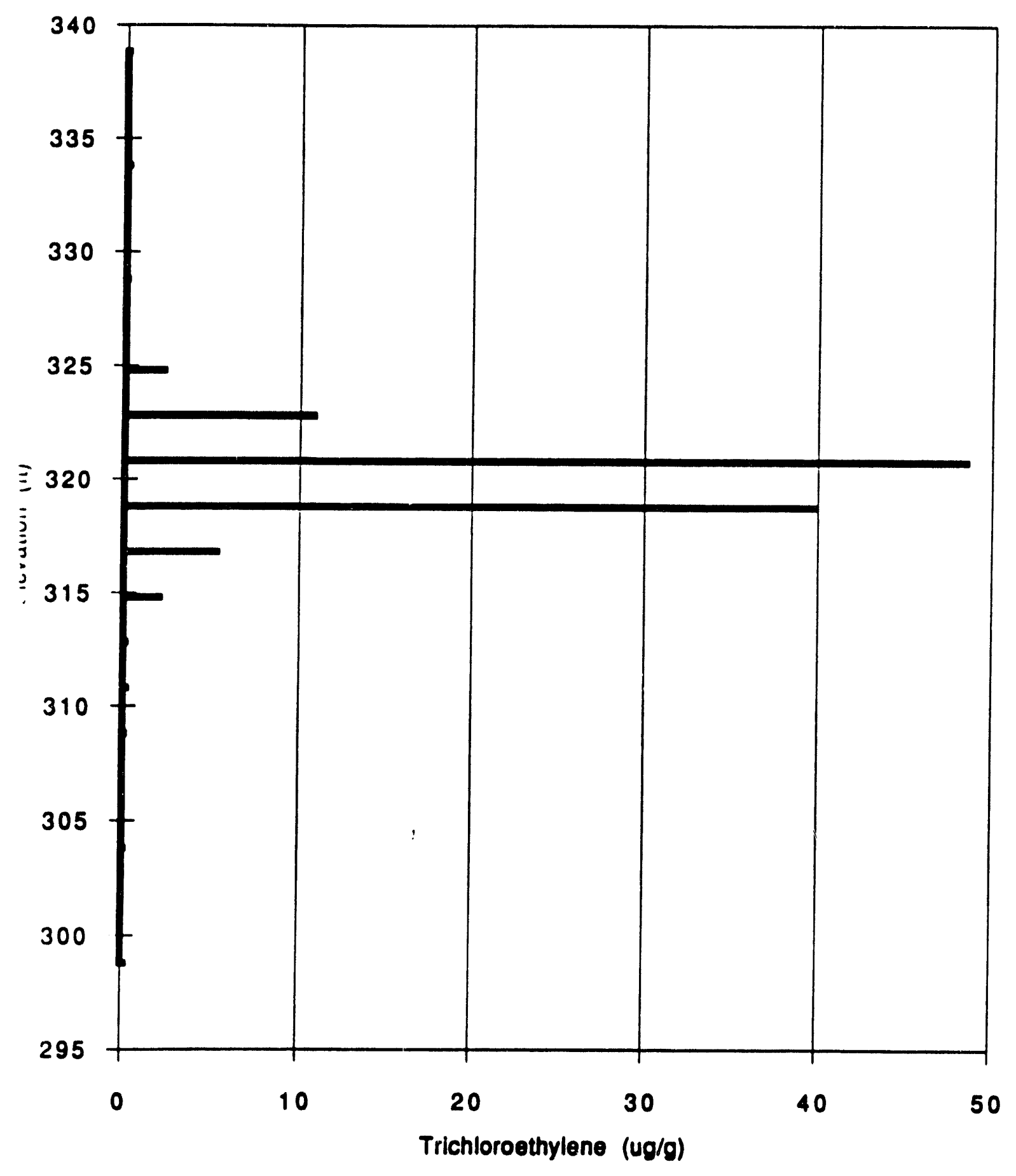




\section{MHB-25 PCE Concentration}

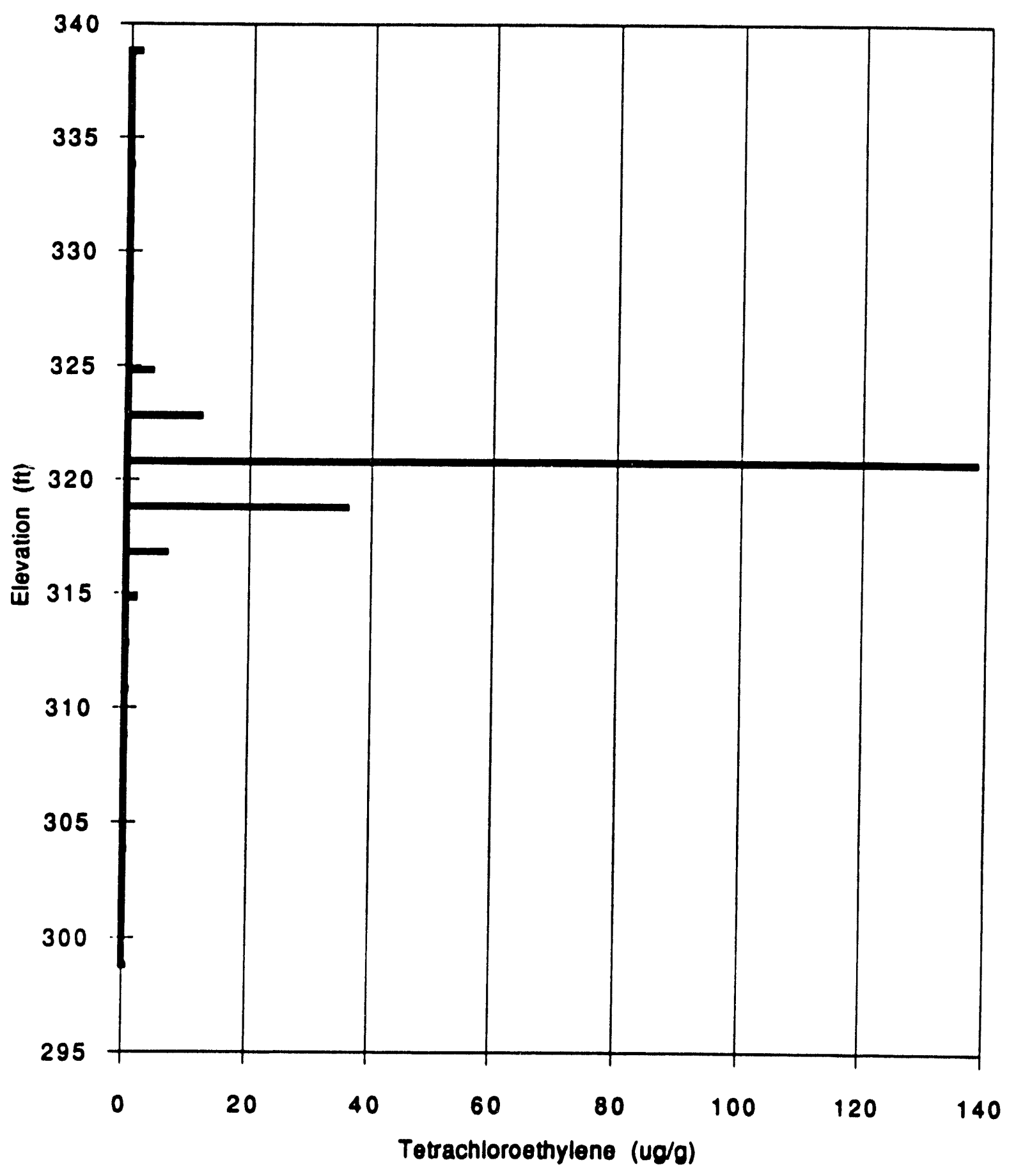




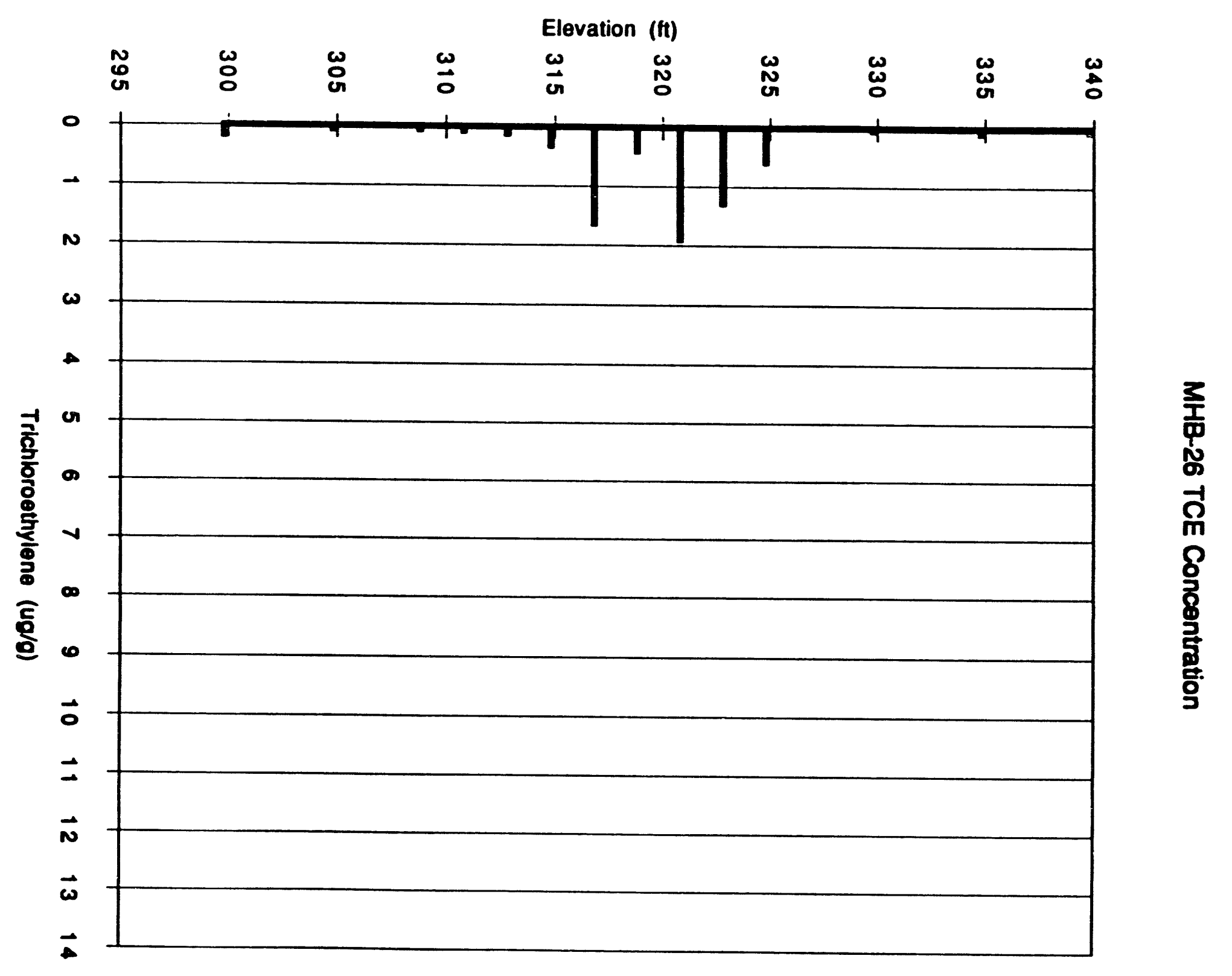




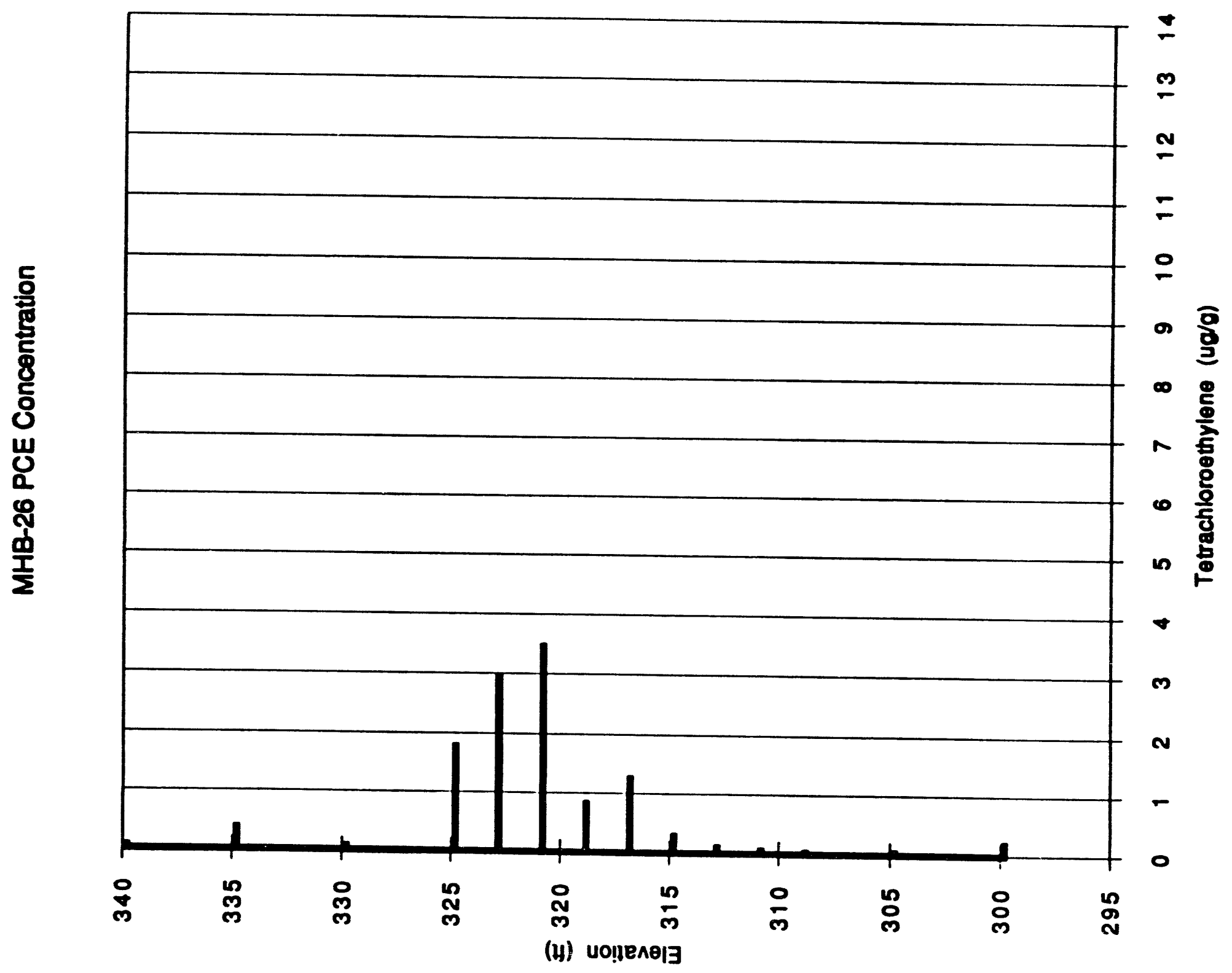


MHB-27 TCE Concentration

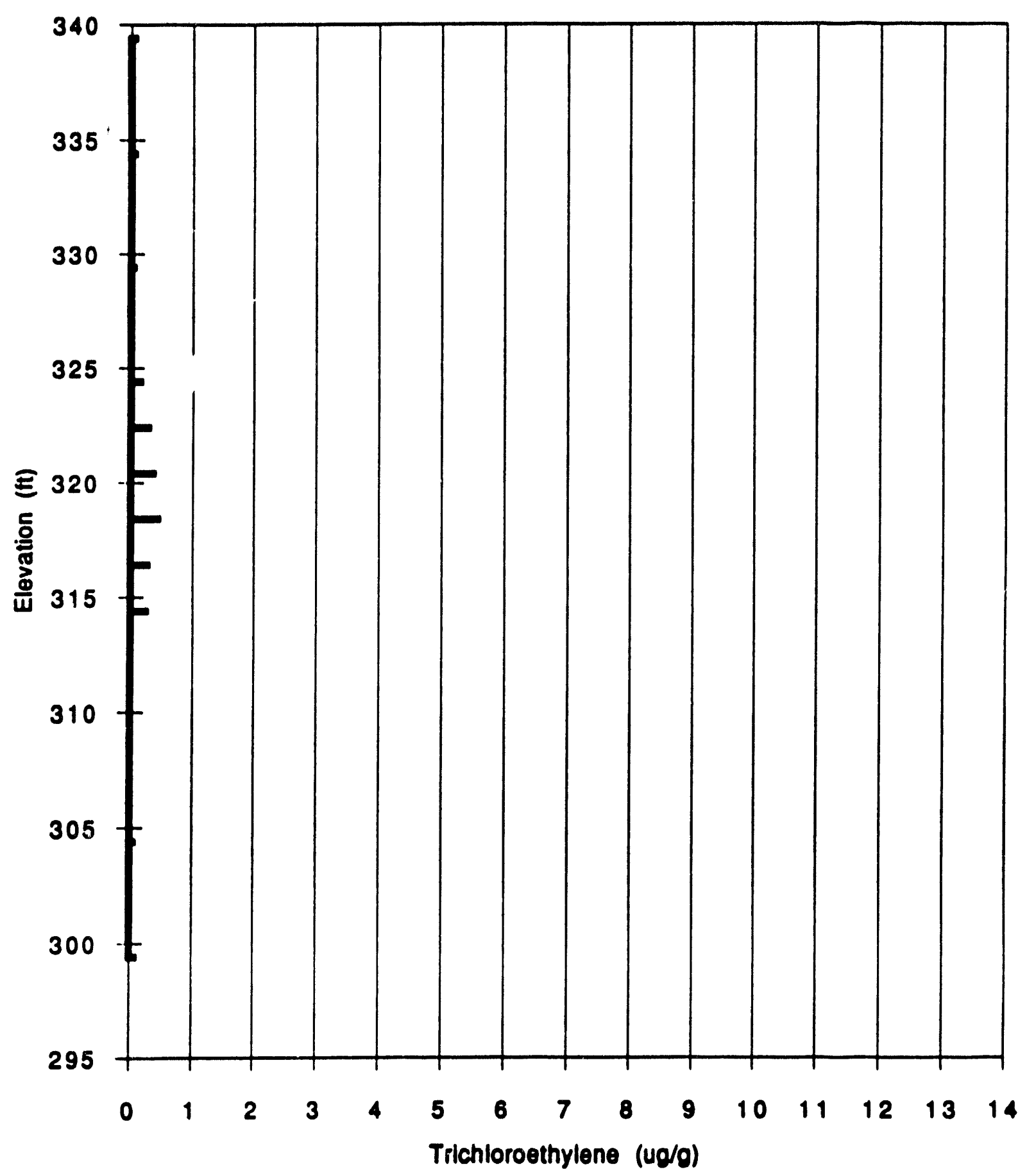




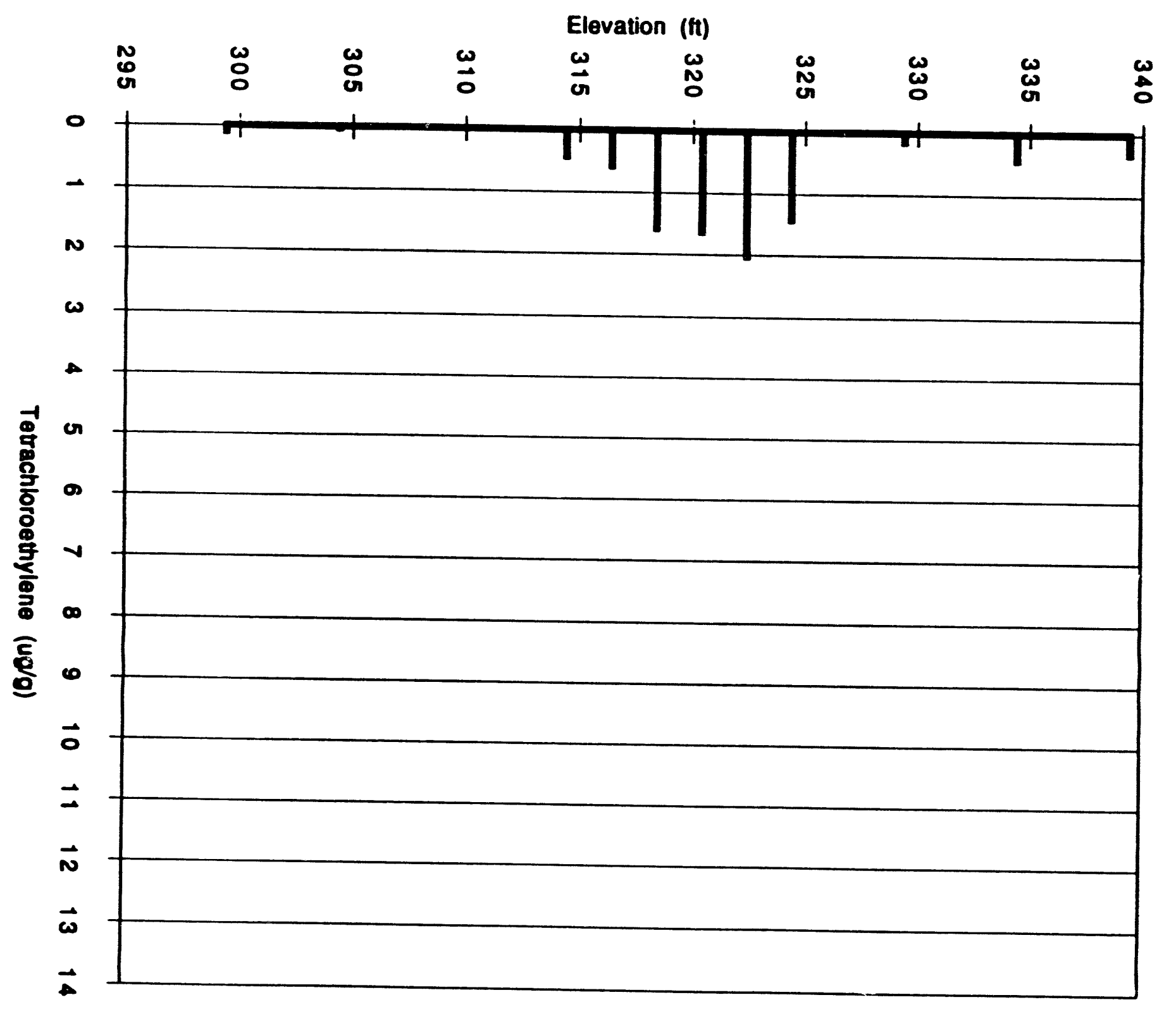




\section{MHB-28 TCE Concentration}

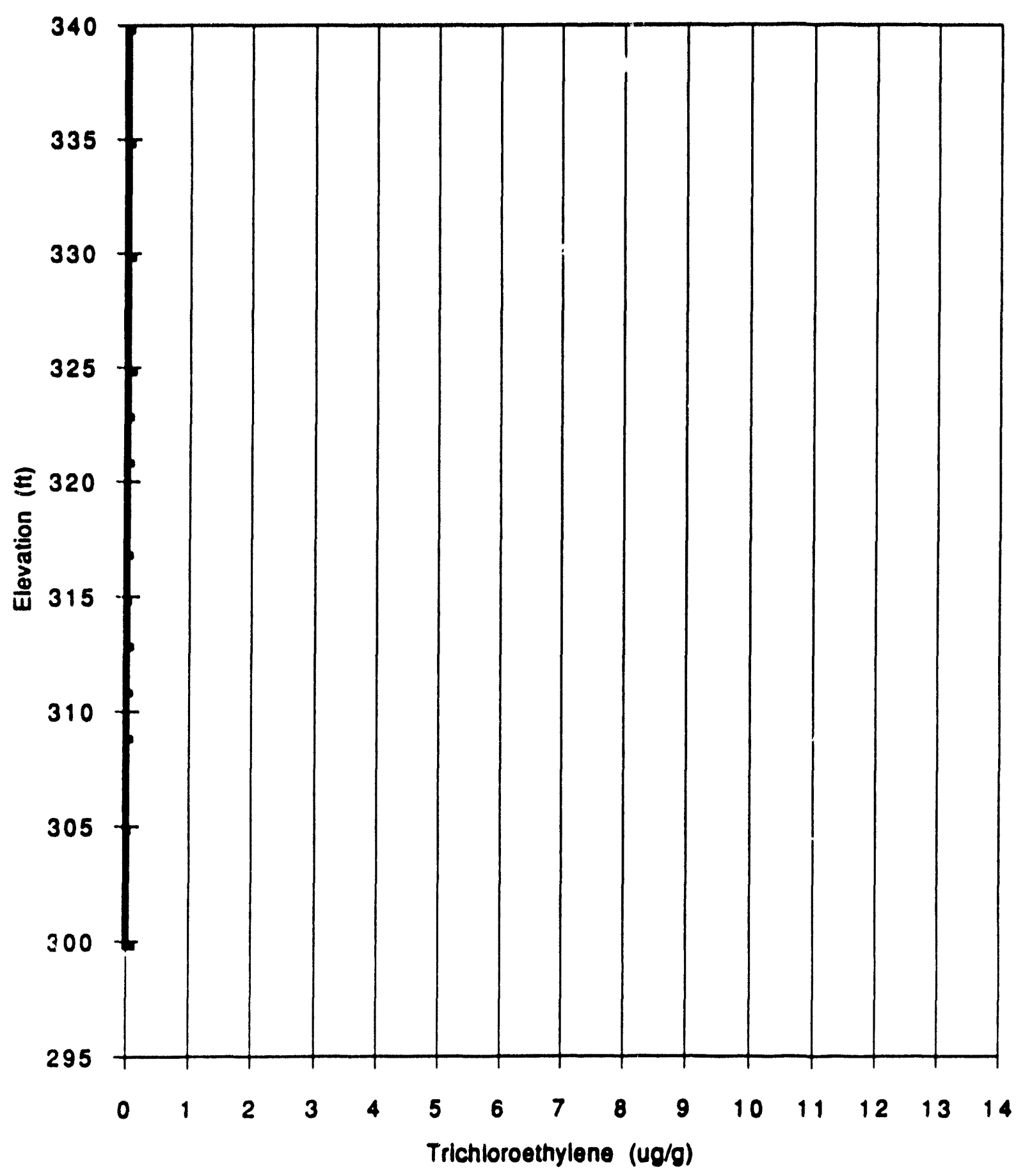




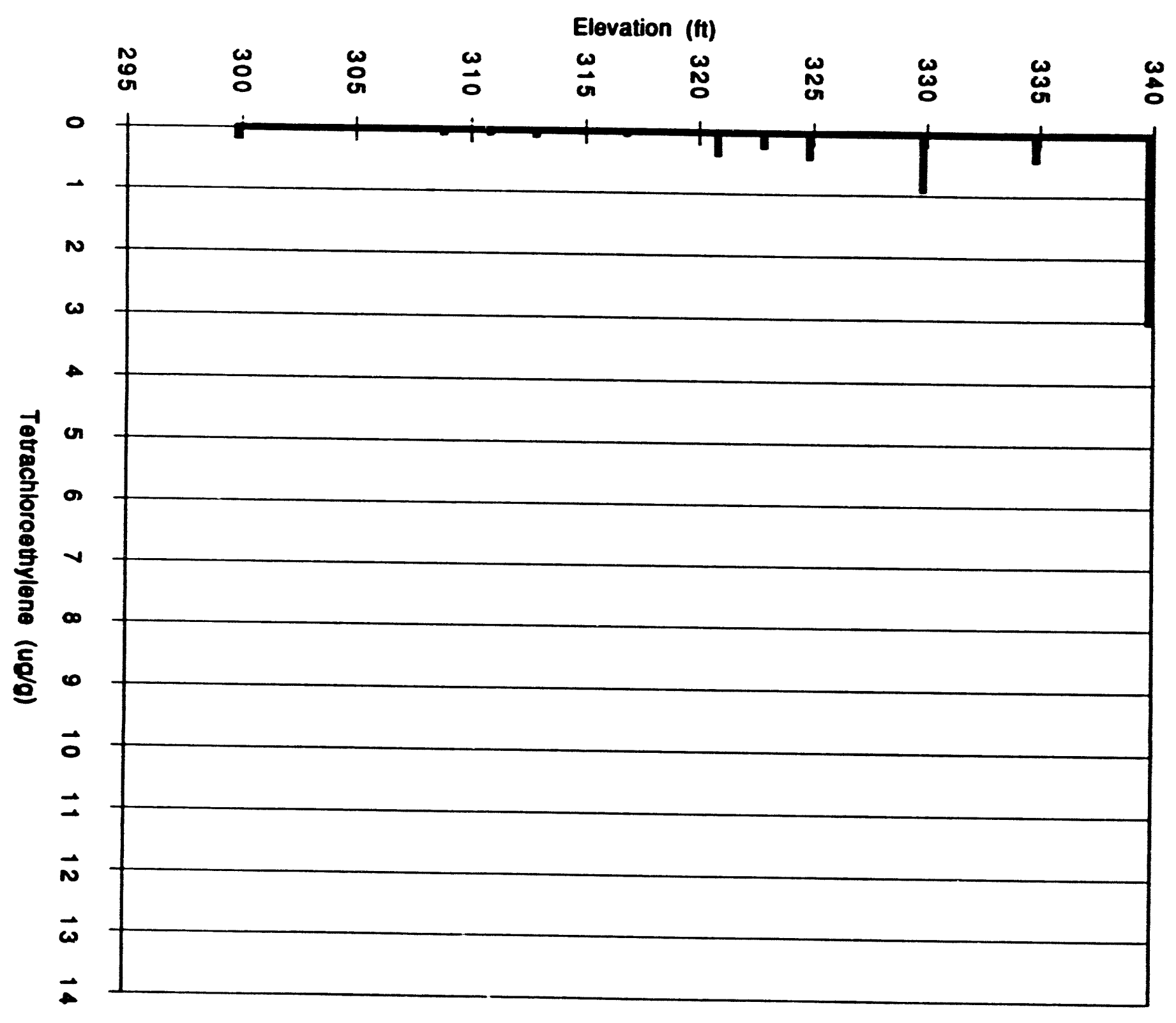




\section{MHB-29 TCE Concontration}

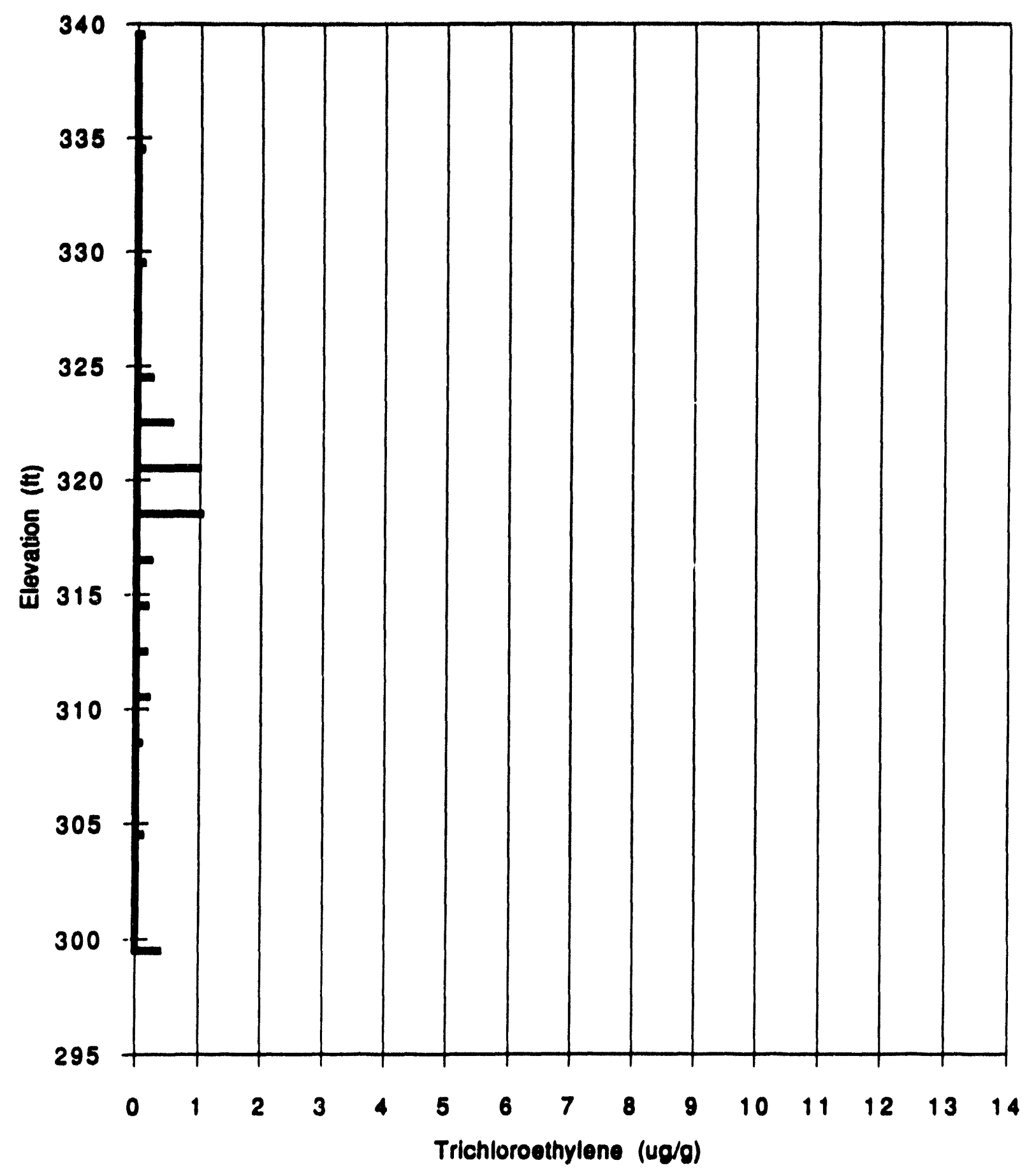


MHV-29 PCE Concentration

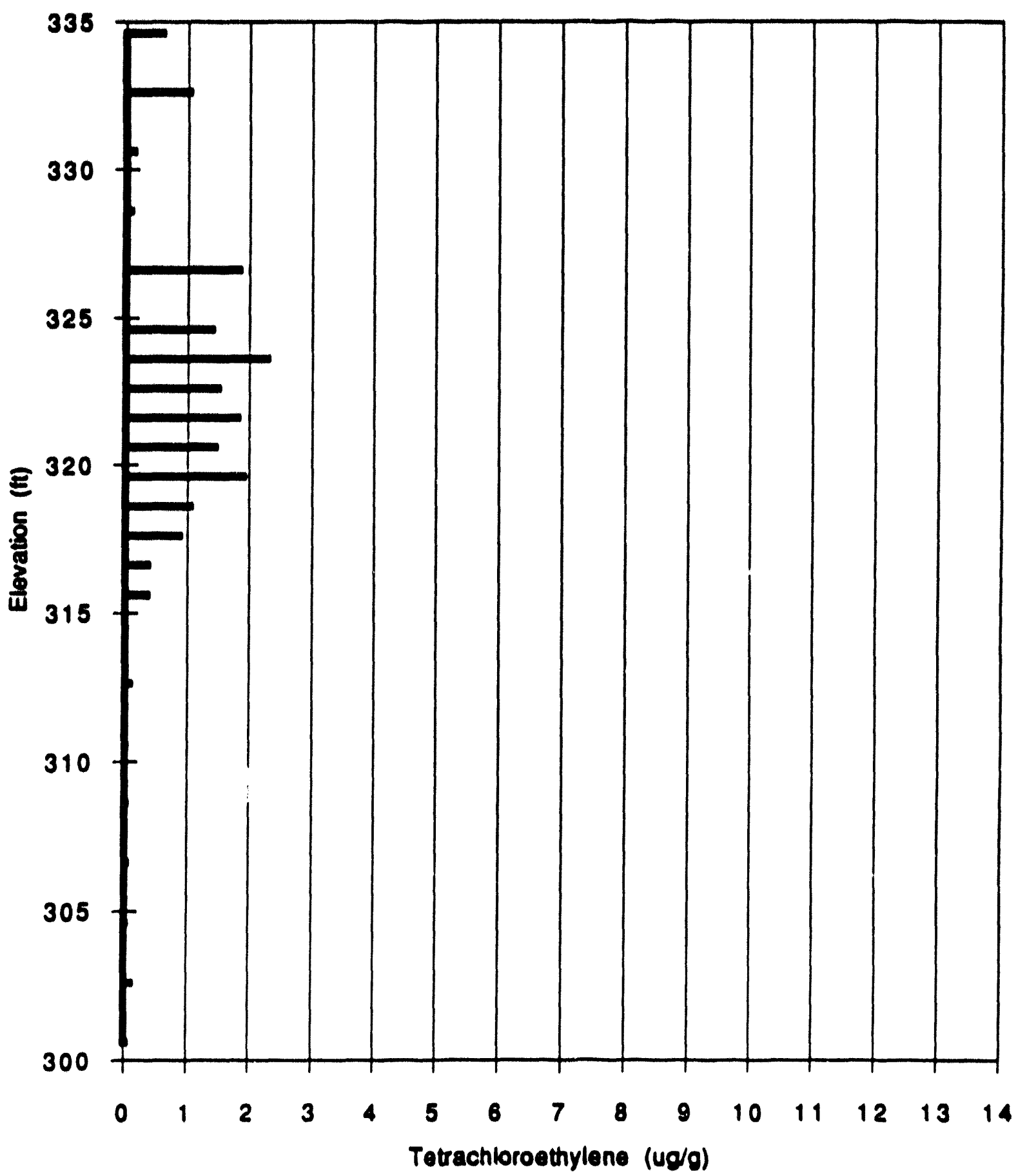




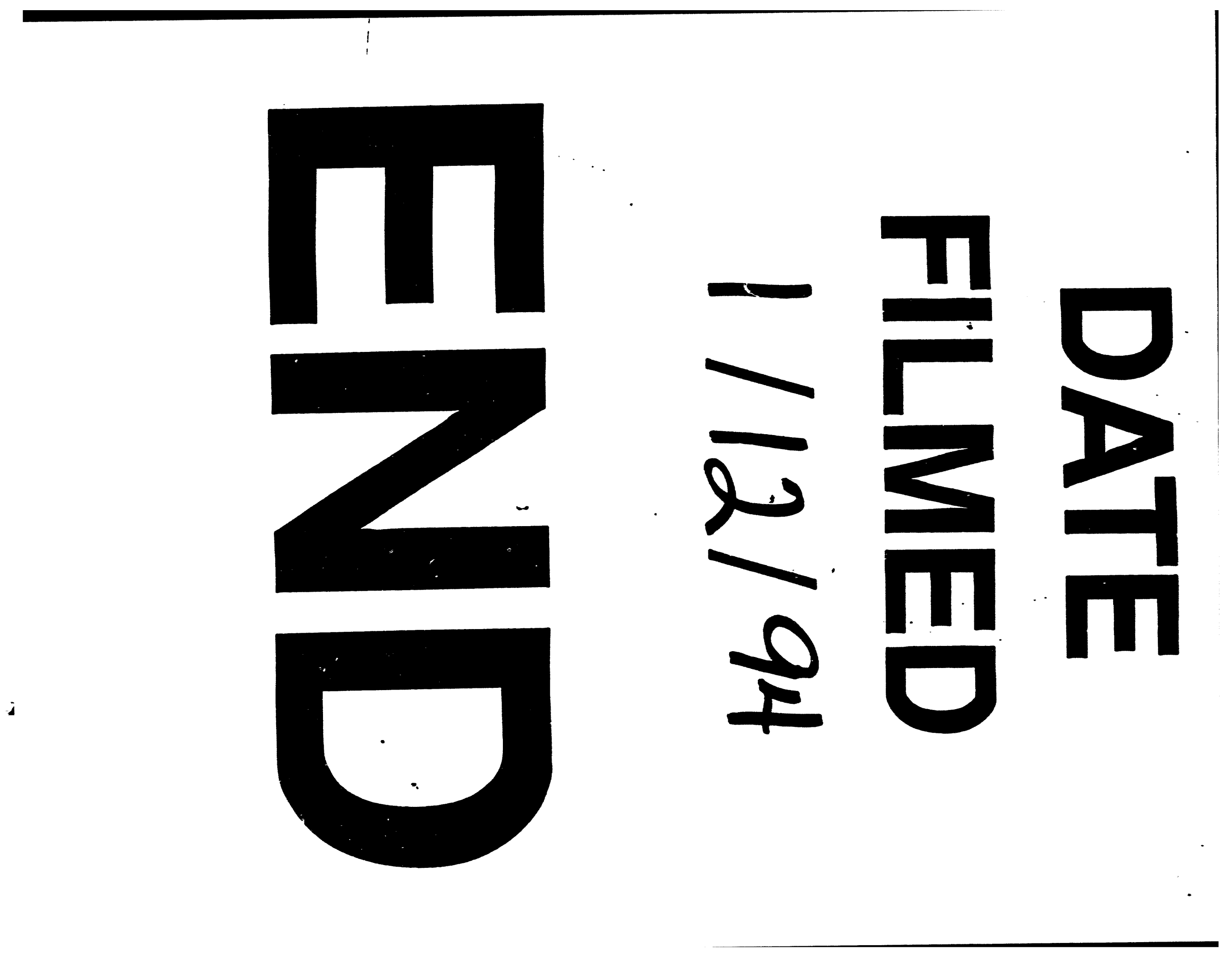


